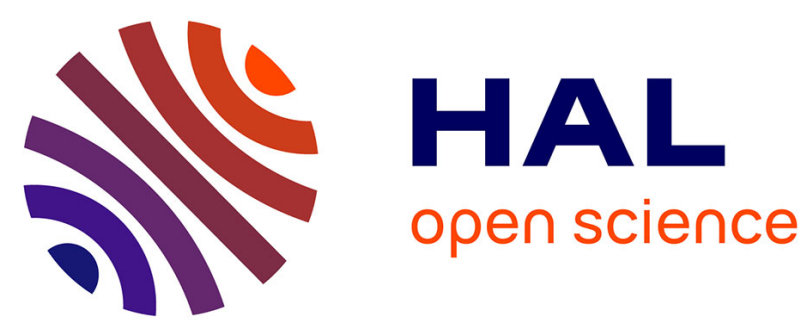

\title{
Macromolecular engineering approach for the preparation of new architectures from fluorinated olefins and their applications
}

Sk Arif Mohammad, Shashikant Shingdilwar, Sanjib Banerjee, Bruno Ameduri

\section{- To cite this version:}

Sk Arif Mohammad, Shashikant Shingdilwar, Sanjib Banerjee, Bruno Ameduri. Macromolecular engineering approach for the preparation of new architectures from fluorinated olefins and their applications. Progress in Polymer Science, 2020, 106, pp.101255. 10.1016/j.progpolymsci.2020.101255 . hal-02871327

\section{HAL Id: hal-02871327 \\ https://hal.science/hal-02871327}

Submitted on 5 Jan 2021

HAL is a multi-disciplinary open access archive for the deposit and dissemination of scientific research documents, whether they are published or not. The documents may come from teaching and research institutions in France or abroad, or from public or private research centers.
L'archive ouverte pluridisciplinaire HAL, est destinée au dépôt et à la diffusion de documents scientifiques de niveau recherche, publiés ou non, émanant des établissements d'enseignement et de recherche français ou étrangers, des laboratoires publics ou privés. 
Macromolecular engineering approach for the preparation of new architectures from fluorinated olefins and their applications

Sk Arif Mohammad, ${ }^{1}$ Shashikant Shingdilwar, ${ }^{1}$ Sanjib Banerjee ${ }^{1 *}$ and Bruno Ameduri ${ }^{2 *}$

${ }^{1}$ Department of Chemistry, Indian Institute of Technology Bhilai, Raipur 492015, Chhattisgarh, India.

${ }^{2}$ ICGM, Univ Montpellier, CNRS, ENSCM, Place Eugene Bataillon, 34095 Montpellier, France

*Correspondence Authors: sanjib.banerjee@iitbhilai.ac.in (SB); bruno.ameduri@enscm.fr (BA)

\title{
Graphical Abstract
}

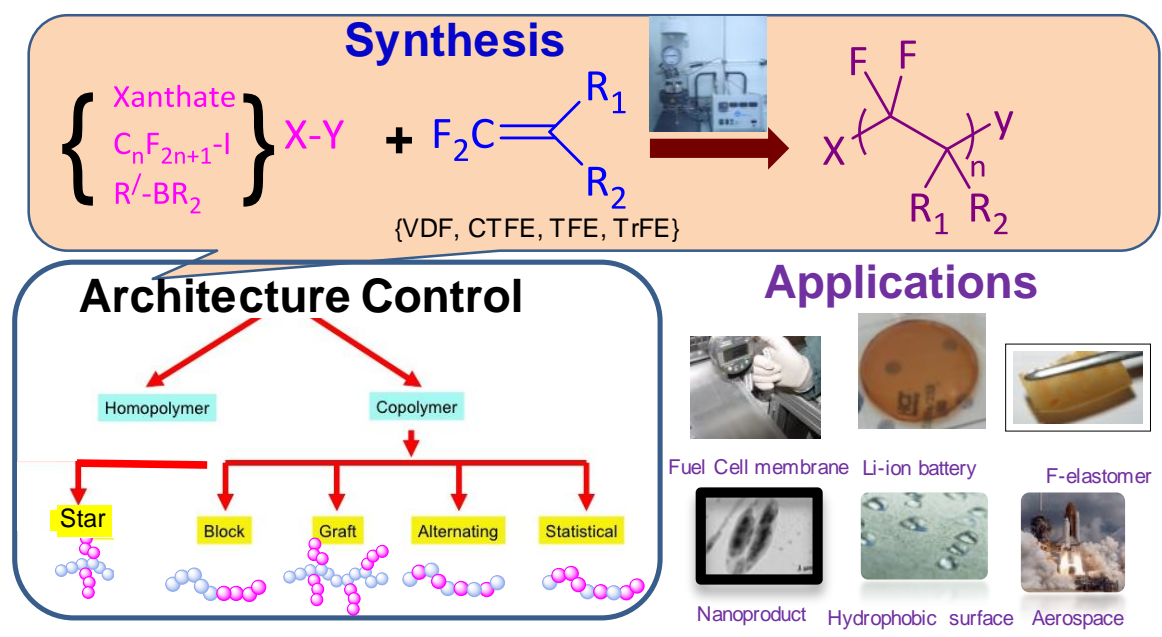

\begin{abstract}
An overview on fluorinated olefins-based architectures prepared by reversible deactivation radical polymerization (RDRP) techniques and applications is presented. Controlled synthesis of well-defined fluoropolymers is discussed as a route to prepare tailored macromolecules of various architectures, such as homopolymers, block copolymers (BCPs), graft copolymers, and star/miktoarms[1]. Primary examples of different strategies of synthesis include (a) Iodine Transfer Polymerization (ITP), (b) Reversible Addition-Fragmentation Chain Transfer /Macromolecular Design via the Interchange of
\end{abstract}


Xanthates (RAFT/MADIX) polymerization, (c) Atom Transfer Radical Polymerization (ATRP), (d) Nitroxide Mediated Polymerization (NMP), (e) Organometallic-Mediated Radical Polymerization (OMRP) and (f) Others systems (based on borinates, Tellurium, and other complexes). Synthesis of BCPs and graft copolymers using these polymerization techniques of vinylidene Fluoride (VDF), chlorotrifluoroethylene (CTFE) and other fluorinated monomers was also discussed, as well as using Copper(I)-catalyzed azide-alkyne cycloaddition (click chemistry). Phase behavior and self-assembly of the fluorinated block copolymers were also discussed. Special attention was devoted to the applications of fluoropolymer architectures in producing thermoplastic elastomers, medical tactile sensors, fuel cells membranes, functional coatings, electroactive polymers (e.g. piezoelectric/ferroelectric/dielectric devices and actuators), high energy storage capacitors, surfactants and composites.

\section{Keywords}

fluoropolymer, reversible deactivation radical polymerization, macromolecular architecture, properties, electroactive polymers, high energy storage

\section{Nomenclature}

1234yf 2,3,3,3-Tetrafluoroprop-1-ene

ATRP Atom transfer radical polymerization

BCP Block copolymer

BDFO 8-Bromo-1H,1H,2H-perfluorooct-1-ene

CMRP Cobalt mediated radical polymerization

CRP (RDRP) Controlled radical polymerization (Reversible Deactivation Radical Polymerization)

CTFE Chlorotrifluoroethylene

CuAAC Copper(I)-catalyzed azide-alkyne cycloaddition

DMA Dimethyl acetamide

DMAEMA 2-(Dimethylamino)ethyl methacrylate 


\begin{tabular}{ll} 
DMF & Dimethylformamide \\
DMSO & Dimethyl sulfoxide \\
EB & Electron beam \\
EGMA & Ethylene glycol methacrylate \\
GPE & Gel polymer electrolyte \\
HFP & Hexafluoropropylene \\
ITP & Iodine transfer polymerization \\
MADIX & Macromolecular design through interchange of xanthate \\
MAF-TBE & tert-Butyl-2-trifluoromethylacrylate \\
MALDI-TOF & Matrix-assisted laser desorption/ionization time-of-flight \\
MMA & Methyl methacrylate \\
NMP & N-Methyl pyrrolidinone \\
NMR & Nuclear magnetic resonance \\
NPs & Nanoparticles \\
OEM & Oxyethylene methacrylate \\
OMRP & Organometallic mediated radical polymerization \\
PFPEs & Perfluoropolyethers \\
PGMA & Poly(glycidyl methacrylate) \\
PMMA & Poly(methyl methacrylate) \\
PS & Polystyrene \\
PSSA & Poly(styrene sulfonic acid) \\
PtBA & Poly(tert-butyl acrylate) \\
PTFE & Polytetrafluoroethylene \\
PVDF & Poly(vinylidene fluoride) \\
RAFT & Reversible addition-fragmentation chain-transfer \\
St & Styrene \\
TFE & Tetrafluoroethylene \\
tBA & tert-Butyl acrylate \\
UF & Ultrafiltration \\
VAc & Vinyl acetate \\
VDF & Vinylidene fluoride \\
\hline
\end{tabular}




\section{Table of Contents}

1. Introduction

2. Fundamentals and developments of Reversible Deactivation Radical Polymerization

2.1. Nitroxide Mediated Polymerization (NMP)

2.2. Atom Transfer Radical Polymerization (ATRP)

2.3. Iodine Transfer Polymerization (ITP)

2.4. Reversible Addition-Fragmentation Chain Transfer /Macromolecular Design via the Interchange of Xanthates (RAFT/MADIX) polymerization

2.5. Organometallic-Mediated Radical Polymerization (OMRP)

2.6. Other systems (based on boranes, Tellurium, and other complexes).

3. Synthesis of well-defined homopolymers

3.1. Synthesis of fluoropolymers via ITP of fluoroalkenes

3.1.1. Synthesis of PVDF using thermal initiators

3.1.2. Photomediated ITP of VDF

3.2. Synthesis via RAFT/MADIX of fluoroalkenes

3.2.1. Synthesis of PVDF by RAFT/MADIX polymerization

3.2.2. PhotoRAFT polymerization of VDF

3.3. Mediated by boranes

3.4. Synthesis of fluoropolymers via OMRP of fluoroalkenes

3.4.1. Synthesis of PVDF using manganese complexes

3.4.2. Synthesis of PVDF using cobalt complexes

3.5. Conclusion

4. RDR copolymerization of F-alkenes

4.1. Synthesis of fluorinated copolymers via ITP

4.2. Synthesis of fluorinated copolymers via RAFT/MADIX 
4.2.1. Copolymers of VDF with 3,3,3-trifluoropropene

4.2.2. Copolymers of VDF with tert-butyl 2-trifluoromethacrylate

4.2.3. Copolymers of VDF with trifluoroethylene

4.2.4. Copolymers of CTFE with n-butyl vinyl ether

4.2.5. RAFT copolymerization of tetrafluoroethylene with isobutyl vinyl ether 4.2.6. Conclusion

4.3. Synthesis of fluorinated copolymers using boranes

4.4. Synthesis of fluorinated copolymers via OMRP and other strategies

4.4.1. Copolymerization of VAc with perfluorohexylethylene

4.4.2. Copolymerization of VAc with CTFE

4.5. Synthesis of fluorinated copolymers via ATRP

4.6. Conclusion

5. Synthesis of fluorinated block copolymers containing VDF and other monomers via RDRP

5.1. Synthesis of VDF-based copolymers via ITP

5.1.1. Synthesis of VDF-based copolymers using thermal initiators

5.1.2. Synthesis of VDF-based block copolymers using photo initiators

5.2. Block copolymers via RAFT/MADIX polymerization of fluoroalkenes

5.2.1. Synthesis of VDF-based block copolymers

5.2.2. Block copolymers based on PVDF and poly(ethylene glycol)

5.2.3. Direct Synthesis of VDF-based amphiphilic diblock copolymers

5.2.4. Direct synthesis and properties of a PVDF- $b$-poly(M) block copolymers

5.2.5. RAFT (co)polymerization of fluoromonomers controlled by trithiocarbonates

5.2.6. Copolymers of CTFE with ethyl vinyl ether

5.3. Block Copolymers via OMRP of fluoroalkenes

5.3.1. Synthesis of VDF-based block copolymers using cobalt complexes 
5.3.2. Cobalt-Mediated Radical Copolymerization of chlorotrifluoroethylene and vinyl acetate

5.4. Block copolymers via ATRP

5.4.1. Synthesis of PVDF containing block copolymers

5.4.2. Synthesis of fluoroalkene-methyl acrylate copolymers

5.5. Block copolymers via polycondensation

5.6. Block Copolymers via copper alkyne azide cycloaddition (CuAAC) "Click Chemistry" 5.7. Conclusion

6. Synthesis of fluorinated graft copolymers

6.1. Graft copolymers via radio grafting

6.2. Graft copolymers from TEMPO or nitroxide groups via NMP

6.3. Graft copolymers via RAFT/MADIX polymerization

6.4. Graft copolymers via ATRP

6.4.1. From PVDF

6.4.2. From CTFE-based polymers

6.4.3. From poly(VDF-co-CTFE) copolymers via the cleavage of $\mathrm{C}-\mathrm{Cl}$ bond in CTFE

6.4.4. From poly(VDF-co-HFP) copolymers

6.4.5. From poly(CTFE-alt-VE) copolymers

6.4.6. From poly(CTFE-alt-VE) copolymers

6.5. Conclusion

7. Synthesis of fluorinated star/miktoarms architecture copolymers

7.1. Synthesis of novel PVDF-based 4-miktoarm star terpolymers

7.2. Synthesis of multi-functional PVDF Graft Copolymers

7.3. Conclusion 
8. Applications

8.1. Thermoplastic elastomers

8.2. Fuel cells membranes

8.2.1. From poly(VDF-co-HFP)- $b$-PSSA diblock copolymers

8.2.2. From graft copolymers prepared from poly(VDF-co-CTFE) copolymers

8.2.3. From copolymers prepared via other strategies

8.3. Electroactive polymers: piezoelectric/ferroelectric/dielectric devices and actuators

8.4. High energy storage capacitors

8.5. Surfactants

8.6. VDF-based amphiphilic diblock copolymers

8.7. Self-assembly

8.7.1. Polymerization-Induced Self-Assembly (PISA) of VDF into PVAc- $b$-PVDF block copolymers

8.7.2. Self-assembly of PVDF-PS block copolymers

8.7.3. Self-assembly of PVDF-b-PEG-b-PVDF triblock copolymers

\subsection{Composites}

8.8.1. Nanocomposites Based on PMMA/PVDF Blends and Multiwalled Carbon Nanotubes

8.8.2. Gold-fluoropolymer nanocomposites

8.8.3. Polymer nanocomposites based on PVDF/PVC doped with graphene nanoparticles

8.8.4. Surface functionalization of $\mathrm{BaTiO}_{3}$ nanoparticles

8.8.5. Surface functionalization of $\mathrm{SiO} 2$ nanoparticle

8.8.6. Nanocomposites Based on PVDF/PMAA/Ni nanocomposites

8.8.7. Nanocomposites containing cobalt ferric oxide 


\subsection{Biomedical applications}

8.10. Gel polymer electrolyte for Li-ion battery

8.11. Functional coatings

8.12. Shape memory polymers

\subsection{Conclusions}

9. Conclusions and Perspectives

10. Acknowledgements

11. References 


\section{Introduction}

Fluorinated (co)polymers have gained lot of interests from both academia and industries as potential smart polymer materials. This observation is because of their i) plethora of unique physical properties such as exhibit excellent thermal/chemical/ageing/weather resistance, high repellent properties towards water, oil and soil low flammability/refractive index/dielectric constants/moisture absorption and improved resistance to oxidative and hydrolytic degradation, and imparts low surface energy (arising from the presence of strong electronegative and low polarizable Fluorine atom in their backbone which makes strong $\mathrm{C}-\mathrm{F}$ bond (its bond energy dissociation $=485 \mathrm{~kJ} \mathrm{~mol}^{-1}$ ) and ii) wide variety of possible morphological architecture/physical states, ranging from completely amorphous, to semicrystalline and to a thermoplastic elastomers (TPE).[2-9] For these reasons, the specialty polymers are widely used in various applications including the automobile industry,[7, 8] aerospace and aeronautics[7, 8], as separators/binders/gel polymer electrolytes in lithium-ion batteries, $[7,8]$ in petrochemical industry (pipes and coatings as liners), for building ultraviolet (UV)-resistant paints and coatings for protecting old heritage monuments.[10,11] backsheets for photovoltaics,[12] organic bulk heterojunction[13] and biomedicine.[14, 15]

An accidental discovery of polytetrafluoroethylene (PTFE) in 1938 by the DuPont Company, prepared via the conventional free radical polymerization (FRP) of a fluorinated monomer, tetrafluoroethylene (TFE) started the journey of fluoropolymer.[16, 17] However, in spite of their widely accepted potential for high value applications, fluoropolymers, specially homopolymers suffer from two major drawbacks: i) poor solubility in common organic solvents due to their highly crystalline nature and ii) difficulty in crosslinking or curing or functionalization due to their lack of specific groups.[18] Significant progress has been made 
to overcome these limitations by producing fluorinated copolymers bearing pendant functionalities[5-8, 18-20]. These functional groups, introduced during the copolymerization stage, induced a high degree of disorder and suppress the high crystallinity of the homopolymers. This led to an enhanced solubility in organic solvents. By proper choice of the functional co-monomers, fluorinated copolymers with unique properties can be prepared for pre-specified high-value applications.

Usually, industrial production of polymers is based on conventional (or free) radical (co)polymerization (FRP) of fluorinated monomers.[2-8] However, FRP techniques suffer from major limitations and produce polymers with uncontrolled molar masses (MWs), broad dispersities (Đs), limited chain-end functionalities, gel fraction, and so on. Recent development of reversible deactivation radical polymerization (RDRP) techniques overcomes these limitations, producing polymers with low dispersity, well-defined molar mass, good control over macromolecular architectures and high chain-end functionality. However, till date, only a handful of RDRP based synthesis strategies has been developed for the synthesis of well-defined fluorinated (co)polymers.[21] Additionally, surface-initiated RDRP[22] techniques provides the opportunity to produce organic/inorganic fluoropolymer hybrids with improved water and oil resistance of the substrate.[23]

Continuous development of different RDRP techniques has made this strategy suitable for producing polymers bearing predesigned end-groups with precisely controlled molar masses, narrow $\doteq$, and well-defined macromolecular architectures (telechelic, block, graft or star copolymers, or dendrimers).[24-28] The development of different RDRP techniques has been discussed in detail in many books and reviews (more than 14,000 articles have been published on RDRP over the last 20 years).[6, 24-29] Thus, this review will not focus on discussing 
extensively the different RDRP techniques[30] and their history of development. For further knowledge, the readers are requested to consult the above-mentioned references. Besides the thermoplastic elastomers invented in the Daikin company[20, 21, 31-33] and as the first polymers produced industrially more than 25 years ago (see section 5), RDRP techniques has started to translate from academic research labs to industries for the development of adhesives, dispersants, lubricants, high-performance elastomers, for applications as novel electrical[34] and biomedical materials, $[15,33,35,36]$ in electronics[37] and in aerospace and automotive industries.[31, 33, 38]

The proposed review starts with an introduction on brief fundamental aspects and developments of different RDRP techniques for the synthesis of fluorinated (co)polymers, their characterizations, properties and applications. Actually, it exclusively focusses on RDRP of fluoroolefins. Basically, the main routes for the synthesis of fluorinated (co)polymers are iodine transfer polymerization (ITP), reversible addition-fragmentation chain-transfer (RAFT) polymerization, organometallic mediated radical polymerization (OMRP), and some controlled by borinates. In addition, a quasi-exhaustive section will be devoted to the synthesis of copolymers via RDRP of two or more fluoroalkenes. Another sub-section reports the synthesis of fluorinated copolymers using Copper(I)-catalyzed azide-alkyne cycloaddition (CuAAC) or "Click Chemistry" strategy. A focus will also be given to the graft copolymers synthesized by various strategies (including "grafting from" or "grafting onto" ways). Finally, properties and relevant applications of these fluorinated copolymers such as thermoplastic elastomers, fuel cells membranes, electroactive polymers (e.g., piezoelectric/ferroelectric/dielectric devices and actuators), high energy storage capacitors, and surfactants will be highlighted and discussed. 


\section{Fundamentals and developments of Reversible Deactivation Radical Polymerization}

Although ITP was pioneered as the first controlled radical polymerization, especially adapted for fluoroalkenes. Since the middle of 1990s, extensive research and development have been carried out on RDRP.[24-28] The origin of the control in any RDRP process is due to the following factors: i) lower concentration of the "active" (macro)radicals compared to the "dormant" chains; ii) reversible fast exchange between living 'active' macroradicals and dormant chains (Scheme 1); iii) evidence of a linear molar mass versus monomer conversion relationship and iv) possibility for chain extension for producing block or graft copolymers. Both features drastically reduce undesirable chain transfer and termination reactions.

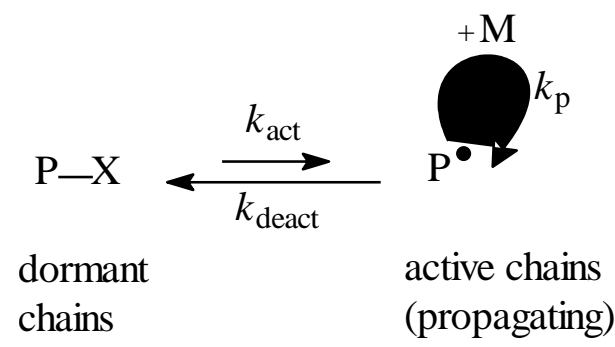

Scheme 1. Reversible fast exchange between active and dormant species in RDRP.

In a pioneering work in 1978, Tatemoto reported ITP of fluoroalkenes.[31-33, 38, 39] Subsequent to this discovery, Otsu's team developed the initiation-transfer-termination method,[40, 41] though that technique was not applied to fluorinated monomers without any specific reason or unsuccessful results. Extensive research on the development of RDRP techniques all over the world has led to the development of nitroxide-mediated polymerization (NMP),[42] atom transfer radical polymerization (ATRP),[26] ITP,[33, 43] RAFT,[44-46] including macromolecular design via interchange of xanthates (MADIX) polymerization,[30, 47] organoheteroatom-mediated radical polymerization based on tellurium, bismuth, 
antimony[48] and organometallic-mediated radical polymerization (ORMP).[49]

Chung and co-workers $[50,51]$ reported the synthesis of end-functionalized fluoropolymers via RDRP using functional borane initiators. Recently, Goto's team $[52,53]$ developed reversible complexation mediated RDRP, but not applied to fluorinated monomers. Tatemoto led the discovery of RDRP of fluorinated monomers.[31-33, 39] The possibility of recombination of the growing macroradicals has hindered the development of RDRP techniques for fluoromonomers.[54] In order to overcome this, various combinations of fluorinated organic compounds have been used as initiators, monomers, chain transfer agents (CTAs) or solvents to achieve RDRP of fluoromonomers.[5, 55] Developments of RDRP techniques including ITP, RAFT polymerization of different fluorinated vinyl and acrylate monomers, including the gaseous ones (such as VDF, CTFE, TrFE) have been reviewed.[21, 56] Though many studies have reported the RDRP of fluoromonomers (acrylates and styrenics) bearing (per)fluorinated side groups,[57] this review focuses on recent exciting discoveries involving the RDRP of fluoroalkenes only, followed by the properties and applications of the resulting copolymers.

\subsection{Nitroxide Mediated Polymerization (NMP)}

NMP is based on a reversible recombination mechanism between the growing propagating (macro)radical and the nitroxide, which acts as a control agent, to yield a (macro)alkoxyamine as the predominant species.[42, 58] Indeed, a nitroxide is a counter radical that does not initiate the polymerization but traps the (macro)radical. This dormant functionality generates back the propagating radical that further reacts onto monomers and the nitroxide by a simple homolytic cleavage upon the temperature increase. When the latter is judiciously chosen, an equilibrium between dormant and active species (Scheme 1), namely the activation-deactivation equilibrium is established. This equilibrium presents the advantage of being a purely thermal process where 
neither catalyst nor bimolecular exchange is required. The polymerization kinetics is governed by both this activation-deactivation equilibrium (with $K=k_{d} / k_{c}$, the activation-deactivation equilibrium constant).[42] To the best of our knowledge, NMP of fluorinated monomers has not been reported till date, except on alternating radical copolymerization of vinyl acetate (VAc) with tert-butyl-2-trifluoromethylacrylate (MAF-TBE).[59]

\subsection{Atom Transfer Radical Polymerization (ATRP)}

In RDRP, the establishment of the required dynamic equilibria can be achieved in two ways: (a) reversible deactivation of propagating radicals to form dormant species that can be intermittently reactivated either in a catalytic manner, ATRP, $[60,61]$ or spontaneously, as in stable radical mediated polymerization, SRMP (with aminoxyl radicals[58] or organometallic species[62]). ATRP[26] is a radical-based process and radicals can proceed from dormant species by several pathways. Mechanistically, halogen atom transfer from alkyl halide to $\mathrm{Cu}^{\mathrm{I}}$ complex can occur via either the outer-sphere electron transfer (OSET) or the inner-sphere electron transfer (ISET), i.e., atom transfer-passing through a $\mathrm{Cu}-\mathrm{X}-\mathrm{C}$ transition state, which is formally also a single electron transfer process. Matyjaszewski[26, 60] suggested the mechanism of ATRP using low ppm Cu activator regenerator by electron transfer and initiator for continuous activator regeneration ATRP, zerovalent metal as reducing metal, electrochemical ATRP or ATRP in aqueous process.

\subsection{Iodine Transfer Polymerization (ITP)}

The development of ITP for preparing fluoropolymers[6, 43] was inspired by the radical telomerization of fluoroalkenes.[5, 63] The concept of RDRP as iodine transfer (co)polymerization of fluoroalkenes was introduced for the first time in the late 1970s by Tatemoto.[32] In this process, the terminal active bond ("pseudo-living" center) of the growing polymer always contains a $\mathrm{CF}_{2}-\mathrm{I}$ bond which originates from the iodine-containing 
CTA and the monomer (Scheme 2). Interestingly, the reactivity of this terminal group always remains almost constant during the entire duration of the polymerization reaction, and even after quenching of the polymerization (allowing post polymerization modification to produce block copolymers).[20, 33, 64-66] Notably, $\mathrm{CH}_{2}-\mathrm{I}$ chain ends are not reactivable and thus leads to generation of dead chain ends and broadening of dispersity.

$$
\begin{aligned}
\mathrm{C}_{n} \mathrm{~F}_{2 n+1^{-I}}+(\mathrm{p}+1) \mathrm{H}_{2} \mathrm{C}=\mathrm{CH}_{2} \stackrel{\text { R or } \Delta}{\longrightarrow} \mathrm{C}_{n} \mathrm{~F}_{2 n+1}-\left(\mathrm{C}_{2} \mathrm{H}_{2} \mathrm{~F}_{2}\right)-\mathrm{CX}_{2} \mathrm{CY}_{2}-\mathrm{I} \\
X=\mathrm{H} \text { or } \mathrm{F} \\
\mathrm{Y}=\mathrm{F} \text { or H }
\end{aligned}
$$

Scheme 2. ITP of VDF in the presence of 1-iodoperfluoroalkanes as the CTA.[21] Reproduced with permission from American Chemical Society.

The control in these polymerizations arises from their high transfer rate $\left(C_{t r}=7\right.$ at $\left.\left.74{ }^{\circ} \mathrm{C}\right),[67]\right)$ compared to the termination rate (which occur exclusively by recombination).[54] Apostolo et al.[64] reported the radical copolymerization of VDF and hexafluoropropylene (HFP) in microemulsion producing well-defined copolymers. At this time Ausimont company (prior to Solexis or Solvay Specialty Polymers nowadays) claimed the "pseudo living branching technology".[68] Peroxide initiators and perfluorinated/chlorofluorinated solvents originally used by Tatemoto and co-workers[32, 33, 39, 65, 66] were subsequently improved by using diiodo and polyiodo compounds as the CTA. Boyer et al.[67, 69] investigated "pseudo-living" ITP of VDF in presence of a series of 1-iodofluoroalkanes as CTA such as $\mathrm{C}_{6} \mathrm{~F}_{13} \mathrm{I}$ and $\mathrm{C}_{6} \mathrm{~F}_{13} \mathrm{CH}_{2} \mathrm{CF}_{2} \mathrm{I}$. According to the authors, the origin of the "pseudo-living" nature is a combination effect of (a) the lability of the $\mathrm{CF}_{2}-\mathrm{I}$ bond (its bond dissociation energy is 220 $\mathrm{kJ} / \mathrm{mol}$ ) and (b) the fast consumption of the CTAs.[67] Beuermann's team developed ITP of fluoromonomers in supercritical carbon dioxide $\left(\mathrm{scCO}_{2}\right)$ in a homogeneous phase using 
$\mathrm{C}_{6} \mathrm{~F}_{13} \mathrm{I}[70]$ or $\mathrm{IC}_{6} \mathrm{~F}_{12} \mathrm{I}[71]$ as the CTA, and prepared PVDFs with low $\oslash(=1.2-1.5)$. The use of $\mathrm{scCO}_{2}$ under high pressure in the ITP was inspired by DeSimone and co-workers' expertise[72] which confirmed that $\mathrm{scCO}_{2}$ can solubilize fluoropolymers, thus inducing a homogeneous medium, in addition to the absence of chain transfer to solvent.

\subsection{Reversible Addition-Fragmentation Chain Transfer /Macromolecular Design via the Interchange of Xanthates (RAFT/MADIX) polymerization}

RAFT has recently emerged as one of the most promising RDRP techniques due to its tolerance towards diverse range of functional groups, and its requirement of less stringent experimental conditions. The fundamentals of RAFT polymerization were reviewed.[44, 45, 73-75] While Haven et al.[76] elaborated on the progress of RAFT polymerization towards the synthesis of discrete or uniform, sequence-defined (co)polymers and mentioned recent developments of RAFT-single unit monomer insertion (RAFT-SUMI) process. RAFT/MADIX polymerization technique was pioneered by the Rhodia Company[31-33, 38] for the RDRP of VAc and has been suitably adjusted to control the polymerization of fluoroalkenes (section 3.2.1).

\subsection{Organometallic-Mediated Radical Polymerization (OMRP)}

Demarteau et al.[49] comprehensively reviewed OMRP processes. In particular, poly(vinyl acetate) (PVAc) with targeted molar masses and low dispersities up to very high monomer conversion and high degree of polymerization was obtained in presence of cobalt acetyl acetonate $\left[\mathrm{Co}(\mathrm{acac})_{2}\right]$ used as a controlling agent. OMRP has recently been extended to other monomers. That review also details the most important synthetic pathways for alkyl-Co 
initiators $\left(\mathrm{R}-\mathrm{Co}^{\mathrm{III}}\right)$ and the influence of the ligand structure on the outcome of the reaction, as well as the possible modes of formation of the radicals, with a focus on the reaction mechanisms.

2.6 Other systems (based on boranes, Tellurium, and other complexes).

Chung[50] studied the radical polymerization of fluoroalkenes (especially VDF) controlled by boranes, the mechanism being supplied in Scheme 3. Results revealed that the borane/oxygen controlled radical initiators is surprisingly effective for initiating polymerization of different fluorinated monomers, including VDF, TrFE and CTFE, at ambient temperature. Such a controlled nature of the polymerization was demonstrated by the production of well-defined polymers with predictable molar mass, low dispersity, formation of end capped polymers and diblock copolymers, and tolerance to various functional groups.

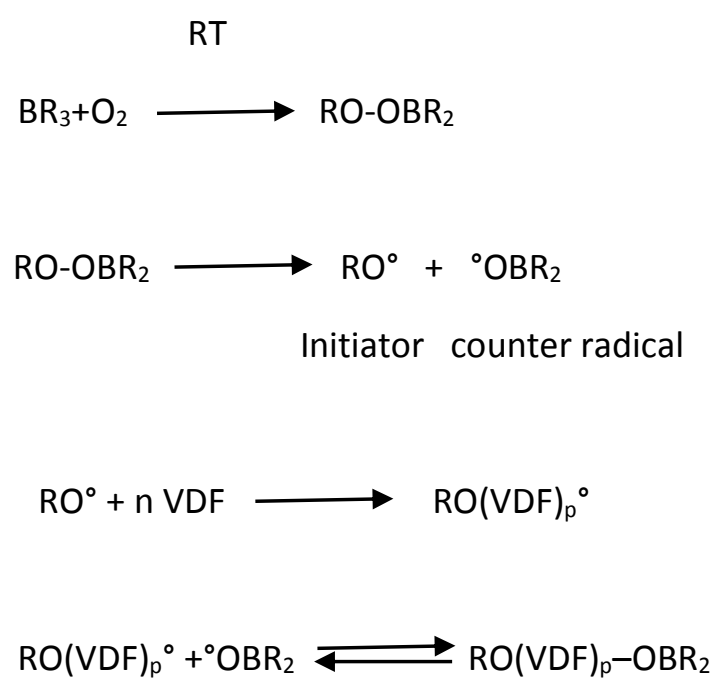

Scheme 3. Synthesis of PVDF mediated by borane/oxygen based radical initiators.[50]

Organotellurium CTAs are usually prepared under basic conditions. The reaction of alkyl or aryl lithium reagents with tellurium metal affords the corresponding organotellanyl lithium species, which reacts with organochlorides, bromides, and iodides to give the desired CTA.[48] Since 
many organolithium reagents and organohalogen compounds are readily available, practical and scalable synthetic routes to organotellurium CTAs have been studied.[48] Yamago's group also demonstrated that organostillbene and organobismuth are also efficient CTAs. Till date, though degenerative transfer in polymerizations of acrylates and styrenes were successful in the presence of these CTAs, there has been no report on RDRP of fluorinated alkenes mediated by organotellurium CTAs.

\section{Synthesis of well-defined homopolymers}

\subsection{Synthesis of fluoropolymers via ITP of fluoroalkenes}

\subsubsection{Synthesis of PVDF using thermal initiators}

Boyer et al.[67, 69] reported two different mechanisms for the ITP of VDF and the kinetics of this reaction were determined in the presence of three different transfer agents. The reactivities of $\mathrm{C}_{6} \mathrm{~F}_{13} \mathrm{I}$ and $\mathrm{C}_{6} \mathrm{~F}_{13} \mathrm{CH}_{2} \mathrm{CF}_{2} \mathrm{I}$ were rather close, and these CTAs were quickly and totally consumed at $75{ }^{\circ} \mathrm{C}$. In addition, the evolutions of $\mathrm{DP}_{\mathrm{n}}$ versus the monomer conversion were similar and linear, thus evidencing the controlled character of the radical polymerization of VDF in the presence of these CTAs. These authors[67] compared ITP of VDF in the presence of two CTAs, $\mathrm{C}_{6} \mathrm{~F}_{13} \mathrm{I}$ and $\mathrm{HC}_{2} \mathrm{~F}_{4} \mathrm{CH}_{2} \mathrm{I}$, the chain transfer constants of which were 7.4 and 0.3 at $74{ }^{\circ} \mathrm{C}$, respectively and later confirmed by simulation by Beuermann's group[77] in water (Figure 1). A fast consumption of the CTA was also noted in $\mathrm{scCO}_{2}$ (Figure 2). 


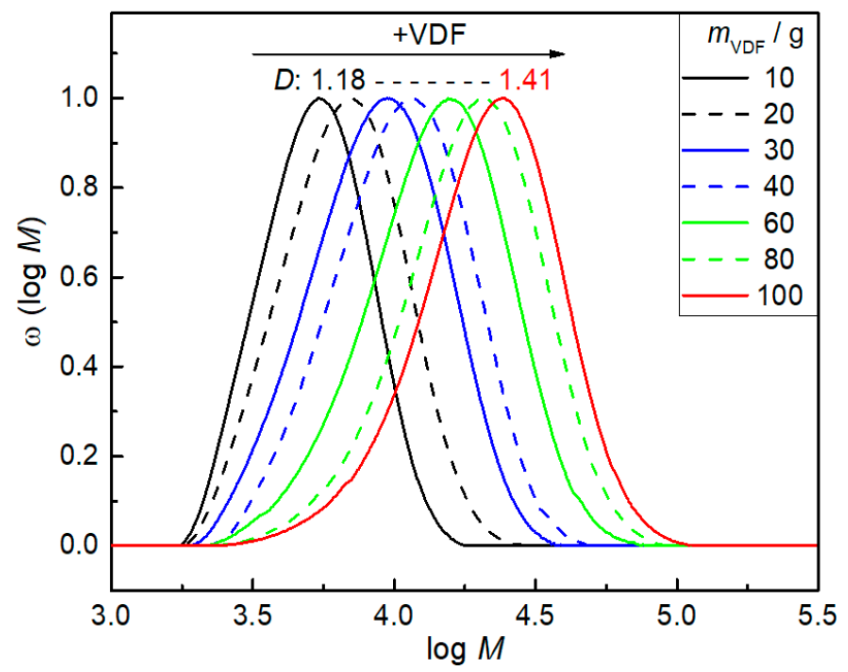

Figure 1. Molar mass distributions of PVDF sampled at the indicated masses of VDF introduced into the reactor. The dispersity increases from 1.18 to 1.41 for ITPs of VDF at $75^{\circ} \mathrm{C}$ and 15 bar with $7.5 \mathrm{mmol} \mathrm{C}_{4} \mathrm{~F}_{8} \mathrm{I}_{2}$.[77] Reproduced with permission from MDPI.

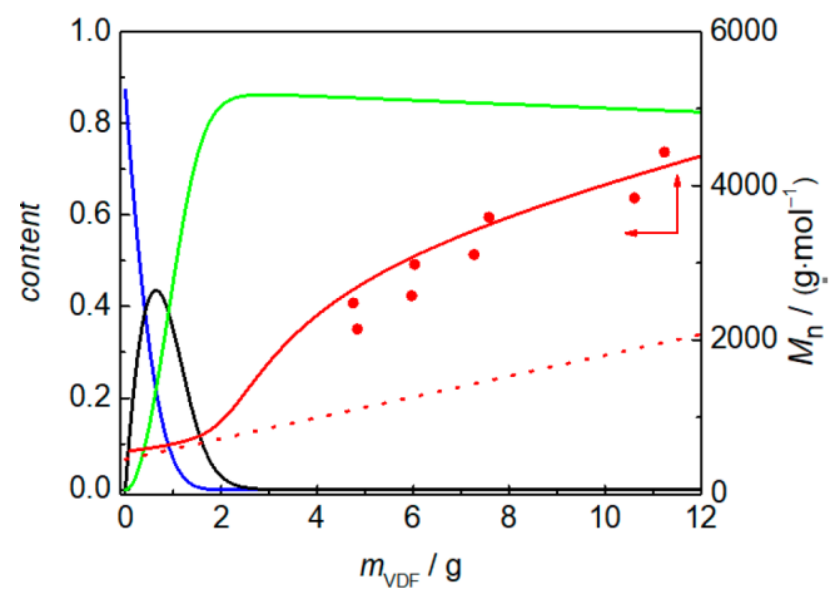

Figure 2. Evolution of the original CTA (blue), the partially transformed CTA (black), fully transformed CTA (green) and number average molar mass (red) during the early stages of ITP of VDF. The experimental points (red dots) and modelled number average molar masses refer to a reaction at 20 bar with $7.5 \mathrm{mmol} \mathrm{I}-\mathrm{C}_{4} \mathrm{~F}_{8}-\mathrm{I}$.[77] Reproduced with permission from MDPI. 
From model ITP reactions of VDF performed in the presence of 1,1,1,3,3-pentafluorobutane, the authors extensively varied experimental conditions in terms of the nature of the radical initiators, time, temperature and initial $[\text { initiator }]_{0} /[\mathrm{VDF}]_{0}$ and $[\mathrm{CTA}]_{0} /[\mathrm{VDF}]_{0}$ molar ratios to tune the yield of the reaction, the average degree of polymerization $\left(\mathrm{DP}_{\mathrm{n}}\right)$, the defect of VDFchaining (Head-to-Head and Tail-to-Tail VDF-VDF additions) (Scheme 4) and the $\mathrm{CX}_{2} \mathrm{I}$ functionality (where $\mathrm{X}=\mathrm{H}$ or $\mathrm{F}$ ).

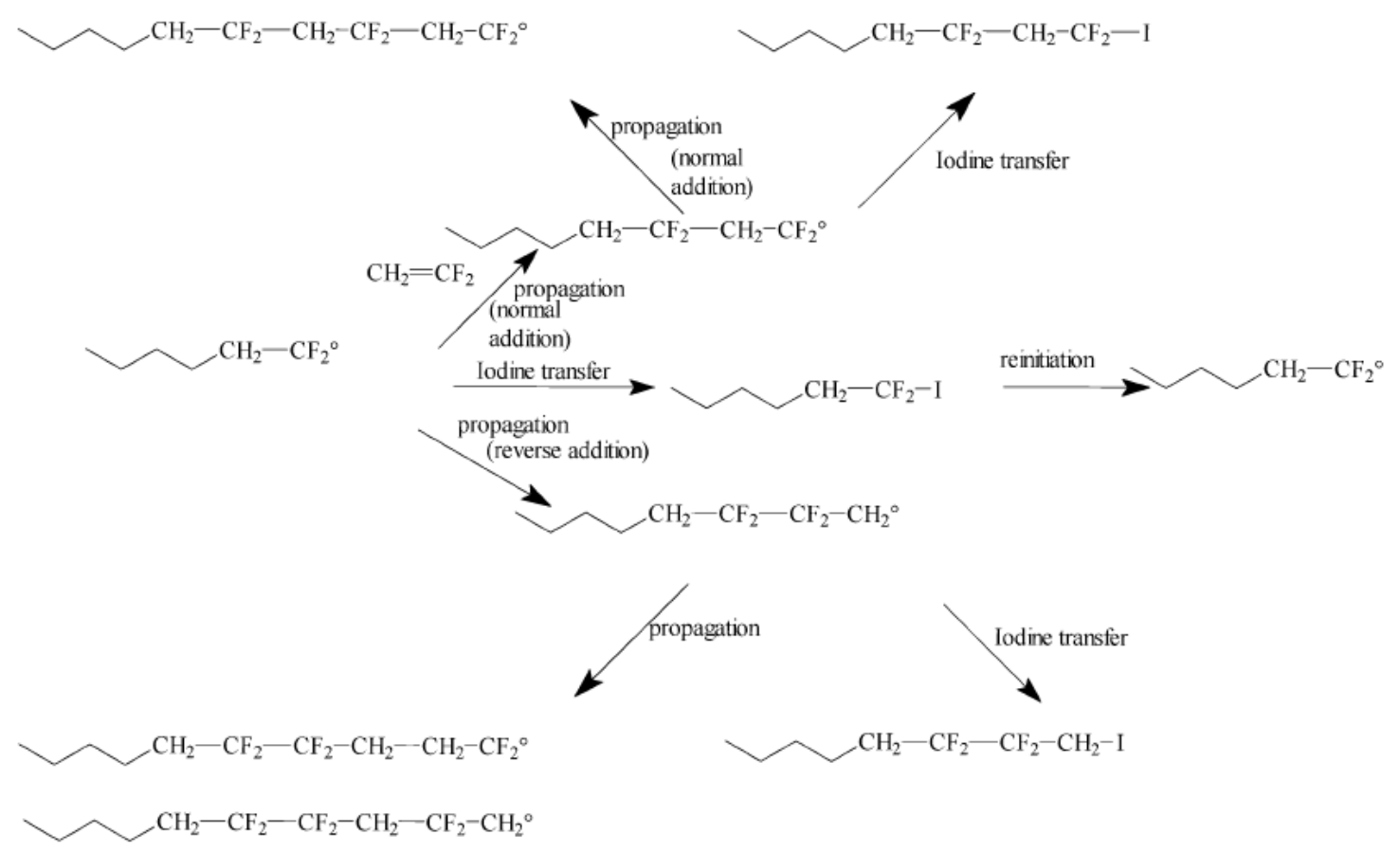

Scheme 4. Different reactions that occur in ITP of VDF.[67] Reproduced with permission from American Chemical Society.

In 2017, Banerjee et al.[78] reported the synthesis of mono $\omega^{-}$Iodo and telechelic diiodo PVDF by ITP (Scheme 5) initiated by a trifluoromethyl radical (generated by perfluoro-3-ethyl-2,4dimethyl-3-pentyl persistent radical, PPFR). Interestingly, from $\mathrm{IC}_{4} \mathrm{~F}_{8} \mathrm{I}$, the formation of $\mathrm{CF}_{3}$ PVDF-I by the direct initiation from ${ }^{\circ} \mathrm{CF}_{3}$ was not observed and ITP of VDF led to I-PVDF-I, exclusively. 


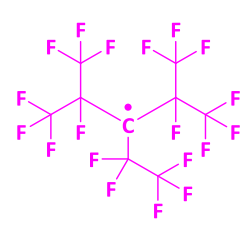

Hyperbranched Persistent Radical PPFR
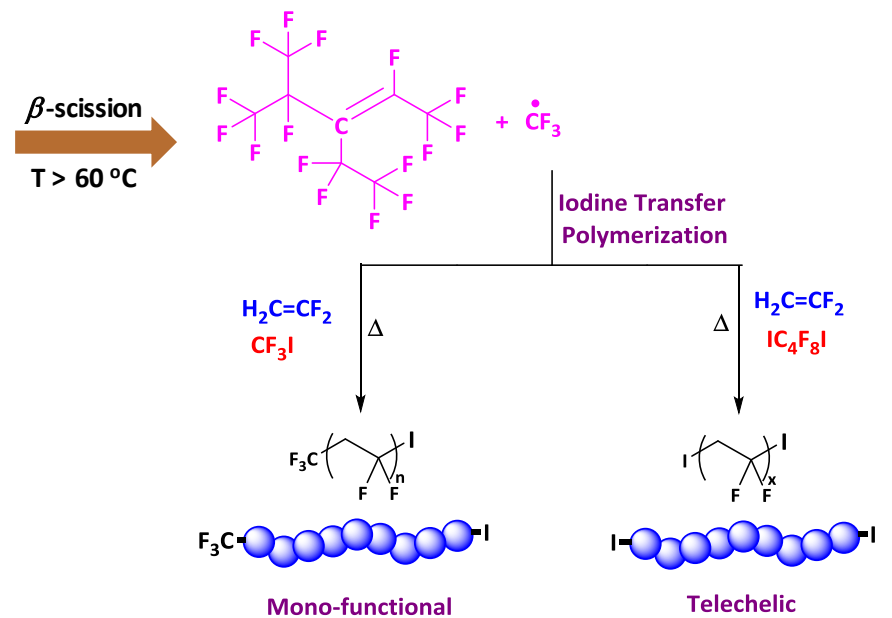

Scheme 5. Synthesis of monoiodo and telechelic diiodo PVDF.[78] Reproduced with permission from American Chemical Society.

\subsubsection{Photomediated ITP of VDF}

Asandei et al.[79] pioneered the photomediated ITP of VDF in presence of $\mathrm{Mn}_{2}(\mathrm{CO})_{10}$ using a variety of alkyl, semifluoroalkyl, and perfluoroalkyl halides $(\mathrm{Cl}, \mathrm{Br}, \mathrm{I})$ as the CTAs. The reactions were carried out in low-pressure glass tubes with a few amounts of VDF, in presence of dimethyl carbonate (DMC). Interestingly, such a system enables the reactivation of PVDF$\mathrm{CF}_{2} \mathrm{CH}_{2}$-I leading to low dispersity PVDF. In general, VDF polymerization initiated by ${ }^{\circ} \mathrm{CX}_{3}$ radicals generated from the metal-free, room temperature, visible-light photodecarboxylation of the corresponding hypervalent iodine (III,V) carboxylates $\left[(\mathrm{RCOO})_{2} \mathrm{IPh} \mathrm{HVICs}\right]$ but Asandei et al.[80] introduced [(bis(trifluoroacetoxy)iodo)]benzene (IFAB), [((diacetoxy)iodo)]benzene, (IDAB), Dess-Martin periodinane, (DMPI) as the most practical and affordable green ${ }^{\circ} \mathrm{CX}_{3}$ and $\mathrm{CX}_{3} \mathrm{I}(\mathrm{X}=\mathrm{F}, \mathrm{H})$ precursors, as exemplified by their use as visible light (Figure 3 ). 


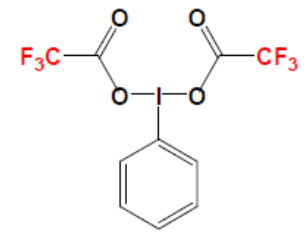

[Bis(trifluoroacetoxy)iodo]benzene (IFAB)

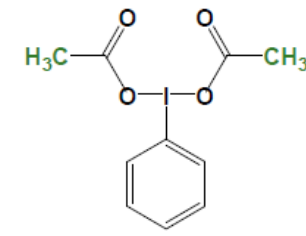

(Diacetoxyiodo)benzene (IDAB)

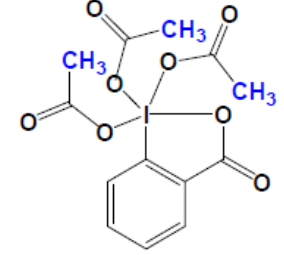

Dess-Martin periodinane (DMPI)

Figure 3. Hypervalent Iodine species with Martin-Arduengo designation [N-X-L] where $\mathrm{N}=\#$ of valence electrons on central atom, $\mathrm{X}=$ central atom, $\mathrm{L}=\#$ of ligands on central atom, used in ITP of VDF.[80] Reproduced with permission from Wiley.

In 2015, the same team also reported the effect of a series of transition metals and ligands in the initiation and control of VDF visible light radical photopolymerization from alkyl and perfluoroalkyl halides in dimethyl carbonate at $40{ }^{\circ} \mathrm{C}$ (Figure 4), as well as in the synthesis of well-defined PVDF block copolymers by a surprising quantitative activation of both PVDF $-\mathrm{CH}_{2}-\mathrm{CF}_{2}-\mathrm{I}$ and $\mathrm{PVDF}-\mathrm{CF}_{2}-\mathrm{CH}_{2}-\mathrm{I}$ chain ends.[81]

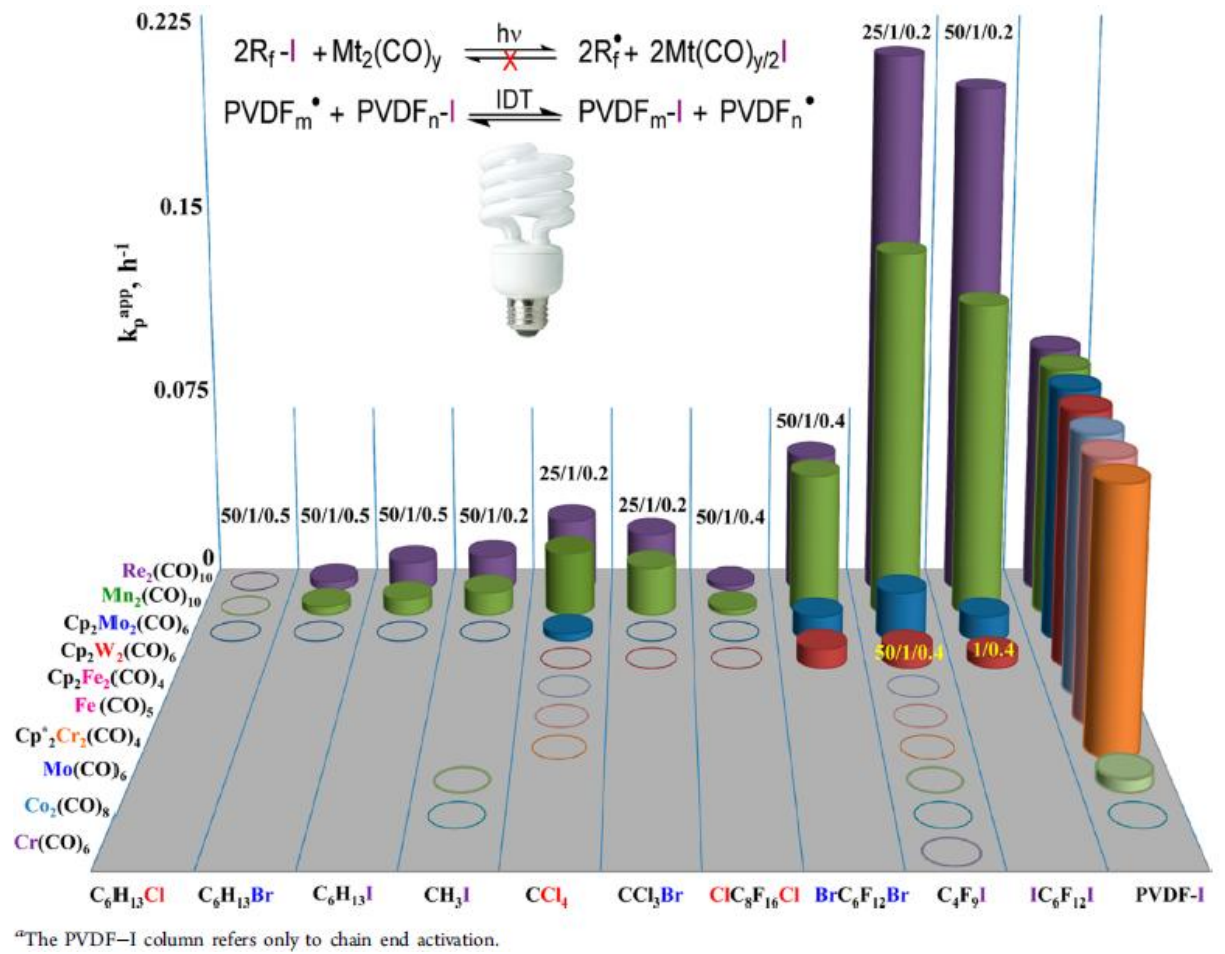


Figure 4. Qualitative dependence of the rate VDF photopolymerization on the nature of the metal carbonyl.[81] Reproduced with permission from American Chemical Society.

In summary, ITP was the first RDRP technique well adapted to fluoroalkenes that led to commercial production in the fluoropolymer industry, pioneered by the Daikin Company and followed by Dupont (now Chemours) and Ausimont (now Solvay Specialty Polymers), for high-performance elastomers or thermoplastic elastomers (see section 9.1). Reactivation of PVDF- $\mathrm{CF}_{2} \mathrm{CH}_{2}$-I end groups could be achieved in the presence of $\mathrm{Mn}_{2}(\mathrm{CO})_{10}$ under visible light. Further academic studies and improvements have also been carried out and interesting fluorinated (co)polymers (Section 4) of various architectures have been designed (Section 5).

\subsection{Synthesis via RAFT/MADIX of fluoroalkenes}

\subsubsection{Synthesis of PVDF by RAFT/MADIX polymerization}

The development of initiating systems to achieve the RDRP of fluoromonomers, is still very challenging. Though RAFT was pioneered in 1998[82], a few studies report RAFT of fluorinated alkenes, the first one being achieved by Kostov et al.[83] on poly(VDF-co-TFP) copolymers in 2011 (see Section 4.2.1). Guerre et al.[84] developed an efficient protocol for the first MADIX polymerization of VDF in DMC using O-ethyl-S-(1methoxycarbonyl)ethyldithiocarbonate (or xanthate) as the CTA (Scheme 6).

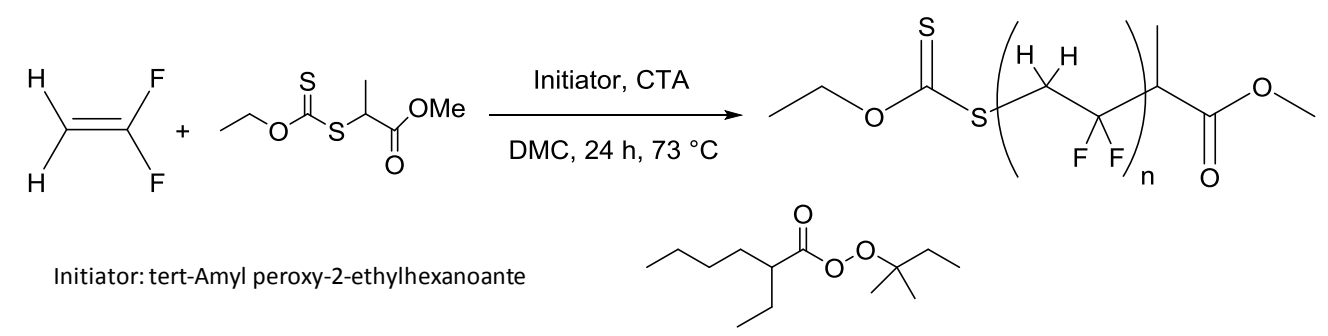

Scheme 6. Synthesis of PVDF by the RAFT/MADIX polymerization of VDF (where DMC 
stands for dimethyl carbonate).[84]

A comprehensive NMR study, including HSQC, HETCOR ${ }^{1} \mathrm{H}-{ }^{19} \mathrm{~F}-\mathrm{NMR}$ and heteroCOSY was performed to determine the end-group functionality of the polymer, mainly $-\mathrm{CH}_{2} \mathrm{CF}_{2}-$ $\mathrm{SC}(\mathrm{S}) \mathrm{OEt}$ and $-\mathrm{CF}_{2} \mathrm{CH}_{2}-\mathrm{SC}(\mathrm{S}) \mathrm{OEt}$.[85] That detailed chain-end analysis was also confirmed by matrix-assisted laser desorption/ionization (MALDI)-time-of-flight (TOF) mass spectrometry (MS) as well as ${ }^{1} \mathrm{H},{ }^{19} \mathrm{~F}$ and HETCOR ${ }^{1} \mathrm{H}-{ }^{19} \mathrm{~F}-\mathrm{NMR}[85]$ to establish that chain transfer reactions to the solvent (DMC) were responsible for a significant loss of CTA and the accumulation of unreactive polymer chains in the reaction medium, leading to a loss of control of the polymerization.[84] This technique allowed us to prepare relatively well-defined PVDF up to $\mathrm{DP}_{20}$, while targeting $\mathrm{DP}_{100-300}$ showed that RAFT of VDF was controlled up to ca. $35 \%$ VDF conversion.[86] For xanthate, a $C_{\operatorname{Tr}(a p p)}$ value of 49 at $73{ }^{\circ} \mathrm{C}$ was obtained[86] confirming the efficient transfer of the RAFT CTA onto PVDF radicals. In addition, RAFT polymerization of VDF can be controlled by aliphatic or aromatic dithiocarbamates.[87]

\subsubsection{PhotoRAFT polymerization of VDF}

Quite a few studies on photoRAFT of fluorinated alkenes have been reported. Yang et al.[88] first studied the RAFT polymerization of 2,2,2-trifluoroethyl $\alpha$-fluoroacrylate (FATRIFE) under thermal conditions and light irradiation and checked performance of four different sulfurcontaining chain transfer agents under thermal condition. Temporal control of the polymerization was demonstrated using light $\mathrm{ON} / \mathrm{OFF}$ experiments.

More recently, the photoRAFT polymerization of VDF was also attempted in the presence of $O$ ethyl-S-(1-methoxycarbonyl)ethyldithiocarbonate as CTA, initiated by visible light.[89] PVDFs with molar masses up to ca. 8000 g.mol ${ }^{-1}$ and low dispersities (1.11-1.49) were synthesized 
(Scheme 7). Kinetics and mechanistic studies revealed that the use of $\operatorname{Ir}(\mathrm{ppy})_{3}$ as a photoredox catalyst resulted in a faster polymerization.

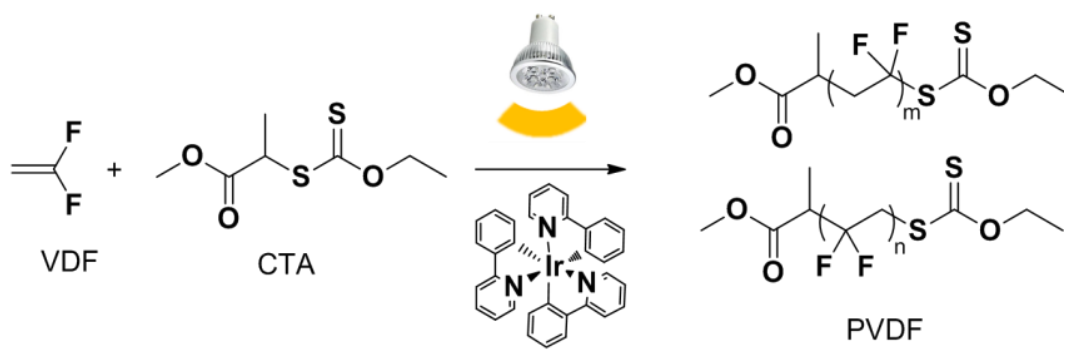

Scheme 7. PhotoRAFT polymerization of VDF in the presence of $O$-ethyl-S-(1methoxycarbonyl)ethyldithiocarbonate.[89] Reproduced with permission from Wiley.

\subsection{Mediated by boranes}

Quite a few studies on boranes, pioneered by Natta[90] and further studied by Sianesi and Caporiccio[91] who depicted the polymerization of vinyl fluoride mediated by alkylboranes in the temperature range of $0-50{ }^{\circ} \mathrm{C}$. Results suggested that enhanced reaction rates and higher polymerization degrees were achieved with alkylboranes, compared to $\mathrm{Cd}, \mathrm{Zn}$, and Be alkyls activated by oxygen. Chung comprehensively used that strategy for the polymerization of many monomers, including fluorinated alkenes such as VDF, CTFE and TrFE in which $\mathrm{RO}^{\circ}$ peroxy radical initiates the polymerization while ${ }^{\circ} \mathrm{OBR}^{\prime}$ acts as a counter radical stabilized by the back donating effect on the radical from $\pi$ orbital.[50, 51, 92, 93] Zhang et al.[92] reported borane mediated synthesis of PVDF for potential application in high energy density capacitors (Scheme 8). The results revealed that the borane/oxygen system controlled radical initiators are surprisingly effective for initiating polymerization of fluorinated monomers. 


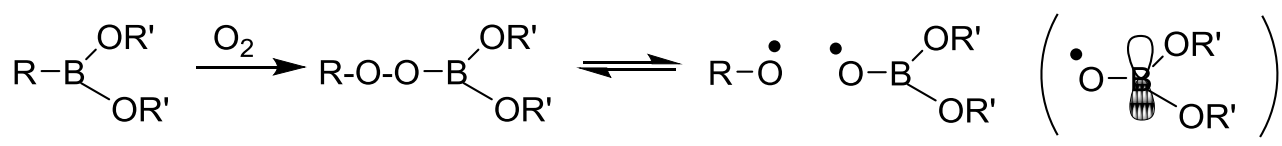

(I)

(III) (IV)

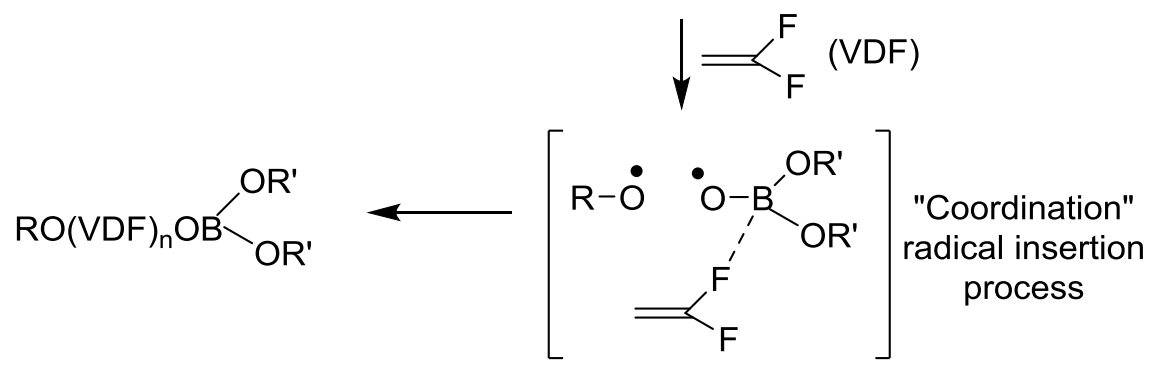

Scheme 8. Initiation process from borane mediated radical polymerization of VDF.[50] Reproduced with permission from Wiley.

Though the controlled character was not evidenced, that technique advantageously can be carried out at room temperature.

\subsection{Synthesis of fluoropolymers via OMRP of fluoroalkenes}

\subsubsection{Synthesis of PVDF using manganese complexes}

Preliminary photochemical activation of fluorinated olefins from $(\mathrm{CO}){ }_{10} \mathrm{Mn}_{2}$ started in mid-70ies leading to non-controlled behavior.[94] In 2012, and as reported in section 3.1.2, Asandei's group smartly photoirradiated the ITP of VDF in the presence of 1 -iodo or $\alpha, \omega$ diiodoperfluoroalkanes[79] showing that $\mathrm{Mn}_{2}(\mathrm{CO})_{10}$ was able to reactivate the dormant PVDF$\mathrm{CF}_{2} \mathrm{CH}_{2}$-I thus evidencing a pseudo-living behavior of that ITP, further leading to original PVDF-b-poly(M) block copolymers. Indeed, Aliwi et al. [94] and Asandei et al. [79] proved that $\left[(\mathrm{CO})_{5} \mathrm{Mn}^{\circ}\right]$ radical, photoproduced from $\left[\mathrm{Mn}_{2}(\mathrm{CO})_{10}\right]$, acts as a catalyst enabling to initiate the radical polymerization of VDF (although it was also reported to initiate those of TFE and CTFE[94]). 
More recently, fluorinated manganese pentacarbonyle complexes, $\mathrm{R}_{\mathrm{F}}-\mathrm{Mn}(\mathrm{CO})_{5}$ (where $\mathrm{R}_{\mathrm{F}}$ stands for $\mathrm{CF}_{3}, \mathrm{CF}_{2} \mathrm{H}, \mathrm{CH}_{3} \mathrm{CF}_{2}, \mathrm{CF}_{3} \mathrm{CH}_{2}$ ), were synthesized to attempt the radical polymerization of VDF via the generation of primary $\mathrm{R}_{\mathrm{F}}{ }^{\circ}$ radicals. The $\mathrm{R}_{\mathrm{F}}-\mathrm{Mn}(\mathrm{CO})_{5}$ bond dissociation energies of the complexes were established theoretically and experimentally (with a suitable fit)[95] in the following decreasing order: $\mathrm{CF}_{3}>\mathrm{CF}_{2} \mathrm{H}>\mathrm{CH}_{3} \mathrm{CF}_{2}>\mathrm{CF}_{3} \mathrm{CH}_{2}$. Three different initiating methods with $\left[\mathrm{Mn}\left(\mathrm{CF}_{3}\right)(\mathrm{CO})_{5}\right]$ (thermal at ca. $100{ }^{\circ} \mathrm{C}$, visible and $\mathrm{UV}$ irradiations) were reported and compared. Unexpectedly, although the $\mathrm{CF}_{3}-\mathrm{Mn}(\mathrm{CO})_{5}$ bond was the most stable, better yields were produced from such a complex that led to PVDF with molar masses reaching 53,000 $\mathrm{g} \cdot \mathrm{mol}^{-}$ ${ }^{1}$ by visible light initiation. Fair $(60 \%)$ to satisfactory $(74 \%)$ PVDF yields were obtained from visible and UV light induced activations, respectively. However, involving $\left[\mathrm{Mn}\left(\mathrm{CHF}_{2}\right)(\mathrm{CO})_{5}\right]$ and $\left[\mathrm{Mn}\left(\mathrm{CH}_{2} \mathrm{CF}_{3}\right)(\mathrm{CO})_{5}\right]$ as radical "initiators" produced PVDF in very low yield (0 to $\left.7 \%\right)$ by both thermal and photochemical initiation, while $\left[\mathrm{Mn}\left(\mathrm{COCF}_{2} \mathrm{CH}_{3}\right)(\mathrm{CO})_{5}\right]$ led to the formation of PVDF with moderate yield (7 to 23\%).[96]

\subsubsection{Synthesis of PVDF using cobalt complexes}

Banerjee et al. [97] reported that organometallic mediated radical polymerization (OMRP) of VDF led to well-defined PVDFs (up to $14,500 \mathrm{~g} / \mathrm{mol}$ ) with low dispersities $(\leq 1.32)$ in presence of oligo(VAc)-Co(acac $)_{2}$ in which organocobalt compound was used as a counter radical. The high chain-end fidelity of the synthesized PVDF was demonstrated by the synthesis of PVDFand PVAc-containing di-and tri-block copolymers. Such a complex could reactivate the PVDF$\mathrm{CF}_{2} \mathrm{CH}_{2}-\mathrm{Co}(\mathrm{acac})_{2}$ end group that reached $20 \%$ only after $60 \%$ VDF conversion (Figure 5). 


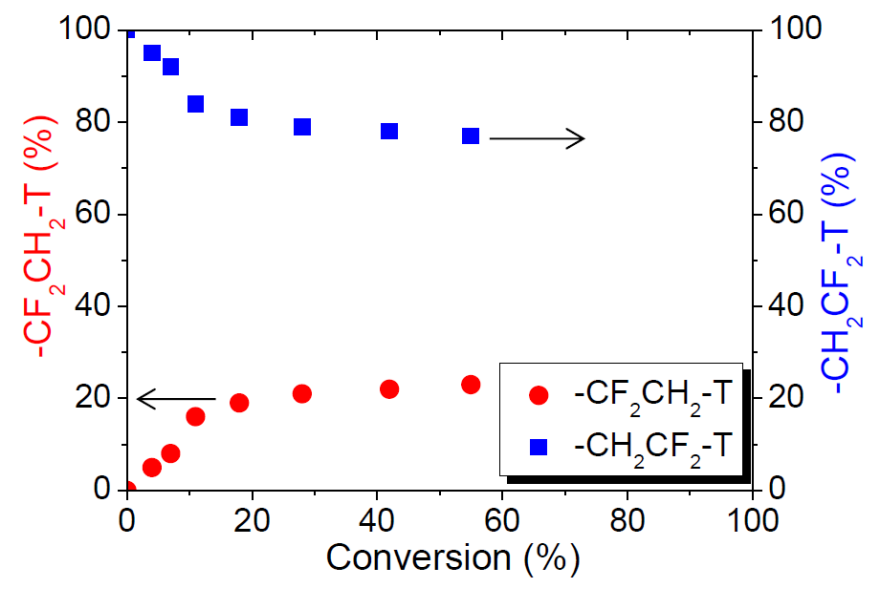

Figure 5. Evolutions of the chain-end functionalities for the OMRP of VDF mediated by oligo(VAc)-Co(acac $)_{2}$ at $40{ }^{\circ} \mathrm{C}$ in DMC after quenching by TEMPO. [VDF $]_{0} /[\mathrm{R}-\mathrm{Co}]_{0}=200$. "T" stands for TEMPO.[97] Reproduced with permission from Wiley.

More recently, Falireas et al.[98] reported the direct synthesis of well-defined PVDF and its block copolymers via OMRP initiated with various peroxides initiators [bis(tert-butylcyclohexyl) peroxydicarbonate was shown to be the most efficient one] and controlled by bis(acetylacetonato)cobalt(II) (Co(acac) $)_{2}$ (Scheme 9). Quenching the PVDF• macroradical by TEMPO allowed us to get stable PVDF-TEMPO that were characterized by SEC and NMR spectroscopy, in contrast to PVDF-Co(acac)2 that spontaneously dimerized under air.

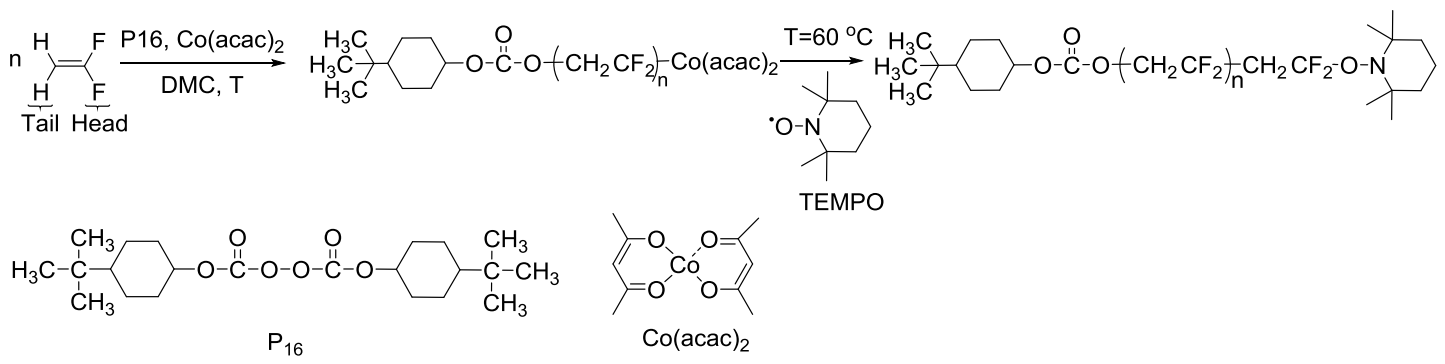

Scheme 9. Preparation of PVDF by OMRP of VDF mediated by Co(acac $)_{2}$ initiated by bis(tertbutylcyclohexyl) peroxydicarbonate (P16).[98] Reproduced with permission from American Chemical Society. 
Poli et al.[99] determined the $\mathrm{X}-\mathrm{C}$ bond dissociation energies (BDEs) for $\mathrm{X}-\mathrm{CH}_{2-n} \mathrm{~F}_{n} \mathrm{CH}_{3-m} \mathrm{~F}_{m}$ molecules $(\mathrm{n}=0,1,2 ; \mathrm{m}=0,1,2,3)$ with $\mathrm{X}=\mathrm{H}, \mathrm{I}, \mathrm{SC}(\mathrm{S}) \mathrm{OEt}, \mathrm{Co}(\mathrm{acac})_{2}$ or $\mathrm{Mn}(\mathrm{CO})_{5}$ (some of which are models of dormant species in the controlled polymerization of ethylene and of certain fluorinated olefins) by ITP $(\mathrm{X}=\mathrm{I})$, RAFT $[\mathrm{X}=\mathrm{SC}(\mathrm{S}) \mathrm{OMe}]$ and OMRP $\left[\mathrm{X}=\mathrm{Co}(\mathrm{acac})_{2}\right]$ by DFT calculation. They provided a suitable agreement with the few experimentally determined values. Calculations were also performed on the simpler $(\mathrm{CO})_{5} \mathrm{Mn}-\mathrm{CF}_{\mathrm{n}} \mathrm{H}_{3}-\mathrm{n}$ molecules $(\mathrm{n}=0,1,2,3)$, for which experimental data are available. The BDE trends as $\mathrm{n}$ and $\mathrm{m}$ vary are different for various $\mathrm{X}$ groups: BDE increased when $\mathrm{n}$ increased (particularly from 0 to 1 ) for $\mathrm{X}=\mathrm{H}$, I and $\mathrm{SC}(\mathrm{S}) \mathrm{OEt}$, but decreased (particularly from 1 to 2 ) for $\mathrm{X}=\mathrm{Co}(\mathrm{acac})_{2}$ and $\mathrm{Mn}(\mathrm{CO})_{5}$. The effective charge analysis indicated that the bond polarity effect on the ionic counterpart of the $\mathrm{BE}$ is a major contribution to these trends. These results rationalized the limited control, especially the accumulation of less easily reactivable dormant species, $\mathrm{PVDF}_{\mathrm{T}^{-}}$ $\mathrm{X}$, generated after an inverted monomer addition, for the polymerization of VDF by ITP and RAFT polymerization. The opposite trend for the transition metal systems (stronger bonds with more Fa-substituted alkyl groups) was in qualitative agreement with the fact that organometallic compounds with fluorinated alkyl groups are generally more stable than their non-fluorinated counterparts, but experimental BDE determinations were limited and affected by large uncertainties.[100] These authors also predicted a better controlled process for this monomer by OMRP mediated by $\mathrm{Co}(\mathrm{acac})_{2}$.

\subsection{Conclusion}

Though ITP is the oldest technique to control the radical polymerization of VDF (and has already led to commercially available fluoropolymers), produced by Daikin, Solvay Specialty 
and DuPont/Chemours, more recent ones have been successfully used such as borane mediated polymerization (even no deep evidence of the pseudo living behavior was reported), RAFT and cobalt mediated radical polymerization. These new techniques offer more versatility leading to various architectures and chain end functional polymers and supported by a theoretical study[99] that compared the efficiencies of $\mathrm{CH}_{3} \mathrm{CF}_{2}-\mathrm{X}$ and $\mathrm{CF}_{3} \mathrm{CH}_{2}-\mathrm{X}$ models where $\mathrm{X}$ stands for $\mathrm{I},-\mathrm{SC}(=\mathrm{S}) \mathrm{OCH}_{3}$ and $\mathrm{Co}(\mathrm{acac})_{2}$. Neither NMP nor ATRP of fluoroalkenes have been reported probably due to the high dissociation energy of $\mathrm{CH}_{2} \mathrm{CF}_{2}-\mathrm{Y}$ bond (where $\mathrm{Y}$ stands for a nitroxide, a bromine or a chlorine group) Further studies on controlled radical copolymerization were also implemented and are summarized hereinafter.

\section{RDR copolymerization of F-alkenes}

Most fluorinated homopolymers (PTFE, PVDF) are crystalline and difficulty soluble in most organic solvents. Thus, copolymerizing their corresponding monomer with other comonomers enables to drastically reduce their crystallinity, allowing them to be soluble in organic media.[28] Several techniques of RDR copolymerization of two or more fluoromonomers have been successfully used, as depicted below.

\subsection{Synthesis of fluorinated copolymers via ITP}

Historically, the Daikin company pioneered the synthesis of thermoplastic elastomers (TPEs) as early as in the late 70ies[31, 32, 101-103] to compete with PS- $b$-polybutadiene- $b$-PS, Kraton ${ }^{\circledR}$ G, from Shell company. Advantageously, some fluorinated TPEs are much more thermostable as well as reported by Tatemoto's group[31-33] at Daikin company which scaled up industrially their production more than 25 years ago. Thus, various TPEs have been designed and summarized in Section 5.

Apostolo et al.[104] carried out detailed kinetics of radical emulsion copolymerization of VDF 
and HFP both experimentally and through the development of an appropriate mathematical model. The authors opined that a reasonable polymerization mechanism usually involve a few kinetic parameters, which all needed to be evaluated independently to improve the accuracy of the model. They assessed the reliability of the developed model with a second independent set of experimental data at operating conditions such as pressure, initial concentration of the initiator, and chain transfer agent. Later Boyer et al.[105] reported the copolymerization of VDF with 2-trifluoromethacrylic acid (MAF) via ITP in presence of 1-iodoperfluorohexane $\left(\mathrm{C}_{6} \mathrm{~F}_{13} \mathrm{I}\right)$ or 1,4-diodoperfluorobutane $\left(\mathrm{IC}_{4} \mathrm{~F}_{8} \mathrm{I}\right)$ as the CTAs, leading to original of $\omega$-iodo or telechelic diiodo poly(VDF-co-MAF) copolymers.[106] The reactions were carried out in water without any surfactant with the assumption that carboxylic acid of MAF unit helped in the aqueous process. However, more recently, the spontaneous addition of water onto MAF leads to $\mathrm{HOCH}_{2} \mathrm{CH}\left(\mathrm{CF}_{3}\right) \mathrm{CO}_{2} \mathrm{H}$ that acts as a surfactant in the emulsion polymerization of VDF.[107] This feature brings insight in the emulsion copolymerization of VDF with MAF. Detailed microstructure analysis of such copolymers was achieved using ${ }^{1} \mathrm{H}$ and ${ }^{19} \mathrm{~F}$ NMR spectroscopy to ascertain the amount of each comonomer in the copolymer, the end-group identification and the molar masses of such resulting copolymers.

In addition, Boyer et al.[108] reported radical terpolymerization of 1,1,2-trifluoro-2pentafluorosulfanylethylene and pentafluorosulfanylethylene in the presence of VDF and HFP by ITP. Both $\mathrm{SF}_{5}$-fluoroolefins do not homopolymerize but copolymerize with VDF (in poor yield) and terpolymerize with VDF and HFP in fair to good yields. ITP was reported to be successful for 1-iodoperfluorofluoroalkenes (Scheme 10).[108]

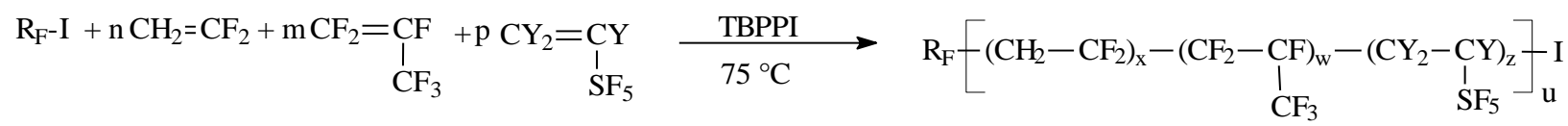

Scheme 10. Radical terpolymerization of 1,1,2-trifluoro-2-pentafluorosulfanylethylene $(\mathrm{Y}=\mathrm{F})$ 
and pentafluorosulfanylethylene $(\mathrm{Y}=\mathrm{H})$ with VDF and HFP by ITP in the presence of $\mathrm{C}_{6} \mathrm{~F}_{13} \mathrm{I}$.[108] Reproduced with permission from American Chemical Society.

Then, the same group[109] reported the synthesis of telechelic diiodopoly(VDF-co-PMVE) copolymers (Scheme 11) by ITP of VDF with perfluoromethyl vinyl ether (PMVE) in presence of telechelic diiodoperfluoroalkane CTAs, using an aqueous process without any surfactant.[110] By means of ${ }^{19}$ F NMR spectroscopy three features were highlighted: (i) VDF is more reactive than PMVE which does not homopropagate; (ii) using $\left(\mathrm{CF}_{2}\right)_{\mathrm{n}}$ signals as a label in the central telechelic poly(VDF-co-PMVE) copolymer enabled to assess their molar masses and (iii) and to unequivocally identify the end-groups (which are exclusively composed of VDF-I). The linear molar mass-monomer conversion relationship confirmed the controlled nature of the polymerization.[109] Indeed, the higher the molar mass of such copolymers, the lower the $\mathrm{CH}_{2} \mathrm{CF}_{2}-\mathrm{I}$ content and the higher the $-\mathrm{CF}_{2} \mathrm{CH}_{2}-\mathrm{I}$ content. Such a latter end group could not reactivate chains because the bond dissociation energy of $\mathrm{CH}_{2}-\mathrm{I}$ bond is higher than that of $\mathrm{CF}_{2}-\mathrm{I} .[109]$

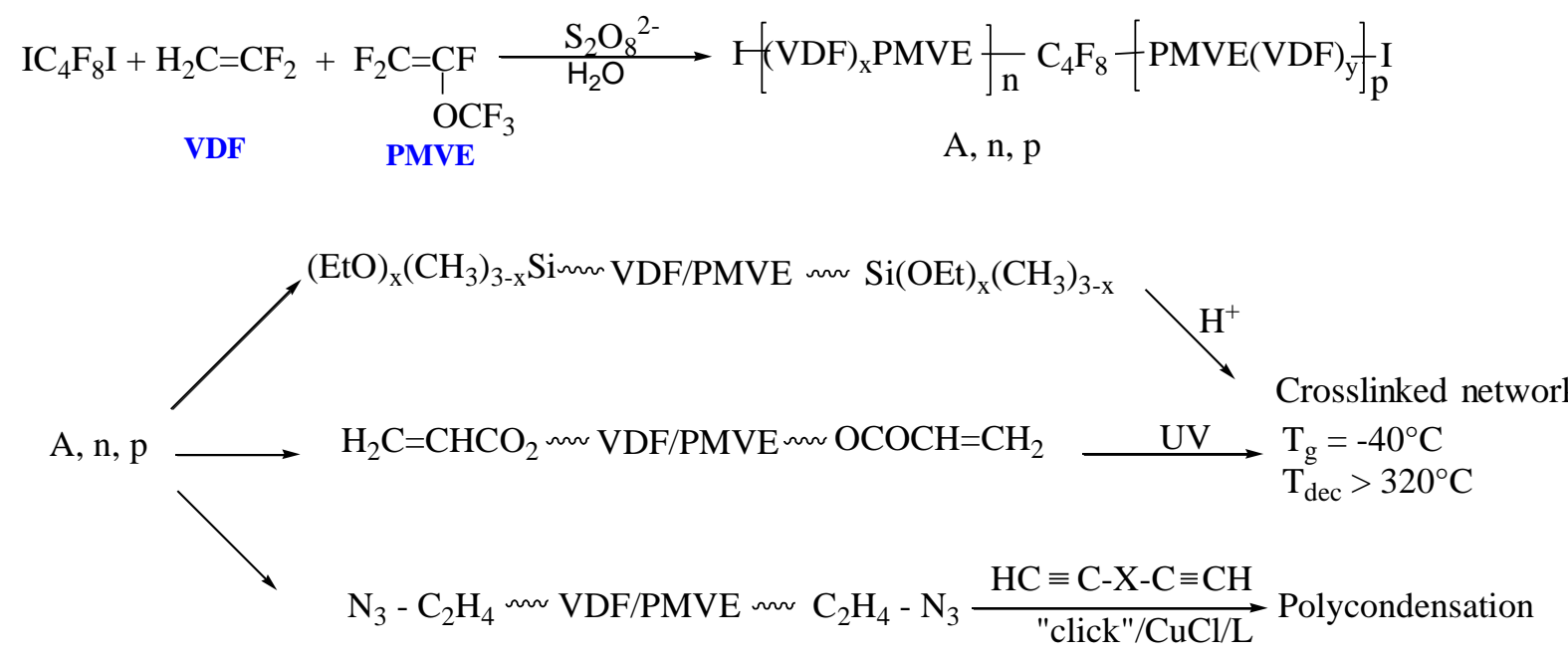

Scheme 11. Iodine transfer copolymerization of VDF with PMVE in the presence of 1,4- 
diiodoperfluorobutane as the CTA, followed by chemical modification and crosslinking.[109] Reproduced with permission from American Chemical Society.

Thus synthesized poly(VDF-co-PMVE) elastomers exhibited a wide range of $T_{g}$ s from -63 to $35{ }^{\circ} \mathrm{C}$ and appreciable thermal stability (decomposition temperatures greater than $250{ }^{\circ} \mathrm{C}$ under air) depending on their molar masses and copolymer compositions. They were also chemically modified into original telechelic bis(hydroxyl),[111, 112] diazido,[113] diacrylates,[111, 114] bis(triethoxysilanes),[115] bis(methyldiethoxysilanes)[115] and further crosslinked to open up new materials endowed with low $T_{g}$ values (as low as $-42{ }^{\circ} \mathrm{C}$ ), satisfactory thermal properties and good chemical inertness to polar and non polar solvents.

Mladenov et al. reported the synthesis of fluorinated copolymers comprising of VDF and HFP via ITP in the presence of 1,6-diiodoperfluorohexane as the CTA.[116] The same group later synthesized fluorinated telomers based on VDF using $\mathrm{C}_{6} \mathrm{~F}_{13} \mathrm{I}$ or $\mathrm{C}_{4} \mathrm{~F}_{9} \mathrm{I}$ as the CTAs for piezoelectric thin films (section 8.3).[117]

Banerjee et al. [118] achieved the first successful iodine transfer copolymerization of VDF with 2,3,3,3-tetrafluoro-1-propene (1234yf) (Scheme 12), followed by post-polymerization modification to prepare functional poly(VDF-co-1234yf) copolymers bearing - $\mathrm{OH}$ end groups.

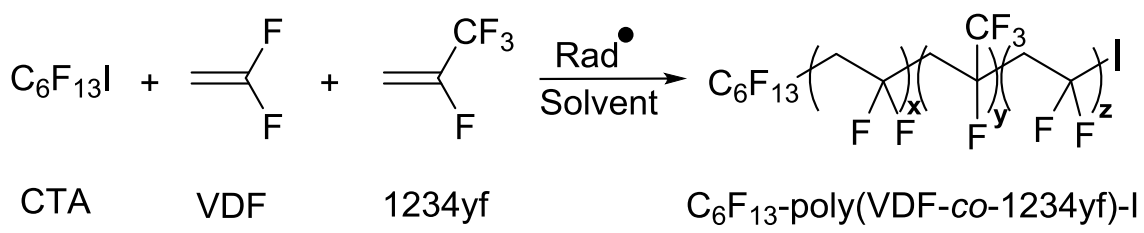

Scheme 12 Radical copolymerization of vinylidene fluoride (VDF) with 2,3,3,3-tetrafluoro-1propene (1234yf) initiated by tert-butyl peroxypivalate (TBPPi).[118] 
Such a reaction was monitored by varying different chain transfer agents (CTAs, such as trifluoroiodomethane, 1,4-diiodoperfluorobutane, and molecular iodine) with/without tert-butyl2-trifluoromethacrylate (MAF-TBE). These authors extensively modified the experimental conditions like the nature of the solvents and CTAs, time, temperature, and initial $[\mathrm{VDF}]_{0}:[\mathrm{CTA}]_{0}:[\text { initiator }]_{0}$ molar ratios, to influence the yield of the reaction, the molar masses, dispersity values, chain-end functionalities, and microstructures of the obtained copolymers. The kinetics of copolymerization highlighted a controlled behavior (Figure 6) and the determination of the reactivity ratios: $r_{V D F}=0.76 \pm 0.34$ and $r_{1234 y f}=1.23 \pm 0.17$ at $74{ }^{\circ} \mathrm{C}$.

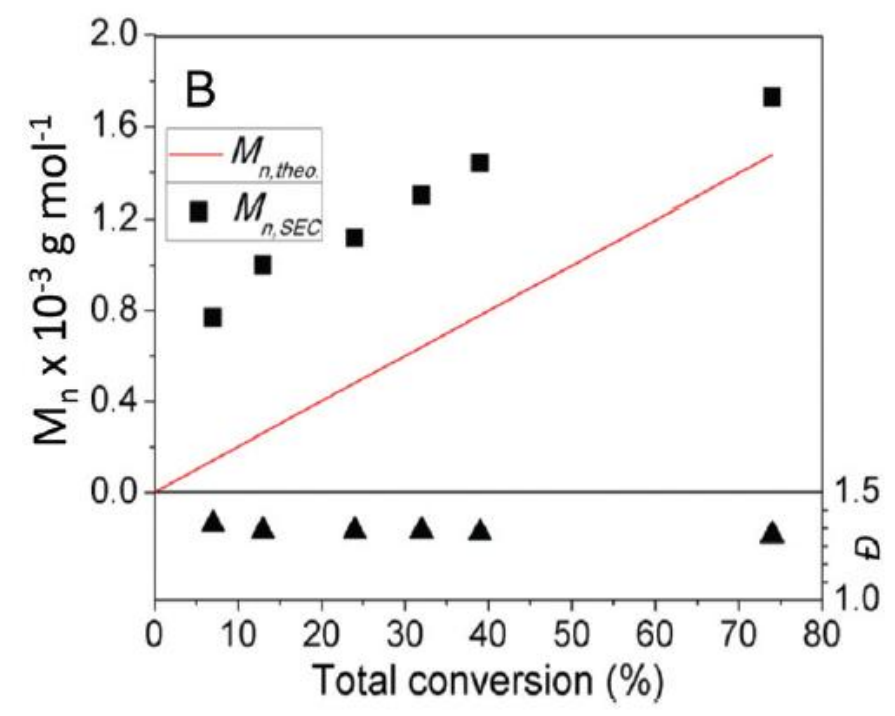

Figrue 6. plot of molar masses $\left(M_{n}\right)$ and dispersities $(\nexists)$ vs. the total conversion for the iodine transfer copolymerization of VDF and 1234yf $\left([\mathrm{VDF}]_{0} /[1234 \mathrm{yf}]_{0}=80 / 20\right)$ initiated by tert-butyl peroxypivalate in the presence of $\mathrm{C}_{6} \mathrm{~F}_{13} \mathrm{I}$ as the $\mathrm{CTA}$ at $74{ }^{\circ} \mathrm{C}$ in 1,1,1,3,3-pentafluorobutane. The red line represents the theoretical $M_{n}$.[118] Reproduced with permission from Royal Society of Chemistry.

Jaye and Sletten[119] developed a facile sodium dithionate initiated copolymerization of $\alpha, \omega-$ diiodoperfluoroalkanes and telechelic dienes to allow high-molar mass alternating copolymers 
(>100 kDa) to be prepared in the presence of oxygen and water with up to 59 wt \%fluorine content (Scheme 13). Through strategic selection of monomers and postpolymerization modifications via addition of azides, thiols, and allyl groups, the fluorous nature of the polymer could be adjusted.

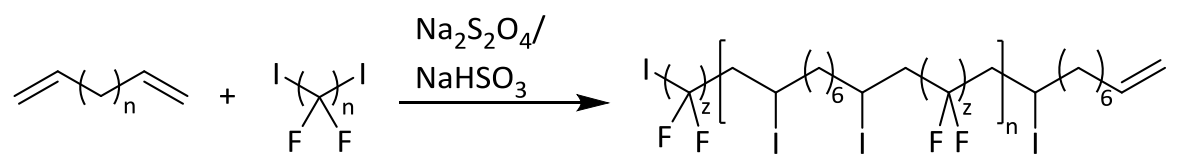

Scheme 13. Iodine Transfer Polymerization of 1,9-decadiene with diiodoperfluoroalkanes to yield fluorinated polymers with varying fluorous blocks.[119] Reproduced with permission from American Chemical Society.

In conclusion, it can be stated that ITP is an important tool to synthesize functional copolymers in presence of various iodo or telechelic diiodo perfluorinated alkanes as the CTA. Early works started 40 years ago have led to commercially available copolymers for various high tech applications (section 9.1). Several monomers (such as MAF, 1234yf, TrFE, HFP and PMVE) have been successfully copolymerized with VDF in a controlled fashion while quite a few achieved with CTFE. In contrast, RAFT/MADIX that is a more recent technique could also be applied on such F-monomers and even on $\mathrm{CF}_{2}=\mathrm{CFX}\left(\mathrm{X}=\mathrm{H}, \mathrm{F}, \mathrm{Cl}, \mathrm{CF}_{3}, \mathrm{OCF}_{3}\right)$.

\subsection{Synthesis of fluorinated copolymers via RAFT/MADIX}

\subsubsection{Copolymers of VDF with 3,3,3-trifluoropropene}

While a fluorinated xanthate containing a $\mathrm{CF}_{3}$ group was pioneered by Monterio et al.,[120] but not involved in the polymerization of fluorinated monomers. Kostov et al. [83] developed another fluorinated xanthate, $\mathrm{C}_{6} \mathrm{~F}_{13} \mathrm{C}_{2} \mathrm{H}_{4} \mathrm{OCOCH}\left(\mathrm{CH}_{3}\right)-\mathrm{SC}(\mathrm{S}) \mathrm{OEt}$, to achieve the MADIX copolymerization of VDF with 3,3,3-trifluoropropene (TFP). These authors further synthesized 
poly(VDF-co-3,3,3-trifluoropropene)- $b$-oligo(vinyl alcohol) block copolymers, with a goal at preparing potentially non-bioaccumulative surfactants (Section 3.2).

Additionally, the same authors also studied the kinetics of telomerization of VDF and TFP in the presence of $\mathrm{C}_{6} \mathrm{~F}_{13} \mathrm{I}$, to determine their reactivity ratios $\left(r_{V D F}=0.28 \pm 0.07\right.$ and $r_{T F P}=2.35 \pm 0.26$

at $75^{\circ} \mathrm{C}$ ) showing a high propagation rate of TFP about that of VDF.[121]

\subsubsection{Copolymers of VDF with tert-butyl 2-trifluoromethacrylate}

Patil et al.[122] successfully attempted the RAFT copolymerization of VDF with tert-butyl 2trifluoromethacrylate (MAF-TBE), initiated by bis(4-tert-butyl cyclohexyl) peroxydicarbonate and controlled by O-ethyl-S-(1-methyloxycarbonyl)ethyl xanthate. The synthesized copolymers were characterized by SEC and also by ${ }^{1} \mathrm{H}$ and ${ }^{19} \mathrm{~F}$ NMR spectroscopy to assess the VDF and MAF-TBE molar percentages, while a linear Mn vs. conversion relationship attested the controlled nature of the copolymerization. As in the example above, chain extension of VAc also confirmed the presence of xanthate end group. The surface properties of the resulting poly(VDFco-MAF-TBE) copolymers revealed tunable hydrophobicity.[123]

\subsubsection{Copolymers of VDF with trifluoroethylene}

The same group developed a similar strategy (Scheme 14) for the RAFT/MADIX copolymerization of VDF and TrFE with the goal to produce electroactive polymers.[124]

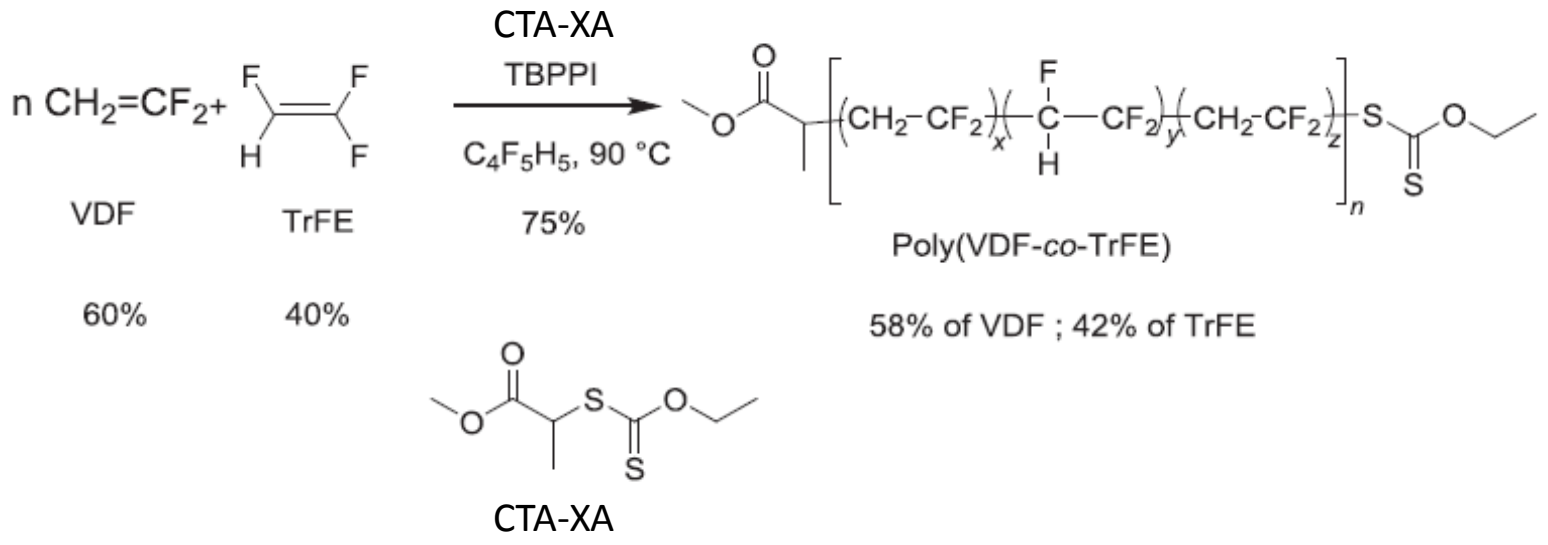


Scheme 14. Synthesis of poly(VDF-co-TrFE) copolymers by MADIX copolymerization of VDF and trifluoroethylene (TrFE) initiated by tert-butylperoxypivalate (TBPPi) using O-ethylS-(1-methoxycarbonyl)ethyldithiocarbonate (CTA-XA).[124]

\subsubsection{Copolymers of CTFE or HFP with n-butyl vinyl ether}

Liu and co-workers reported the MADIX alternated copolymerizations of CTFE[125] (or HFP[126]) with n-butyl vinyl ether (BVE) initiated under ${ }^{60} \mathrm{Co} \gamma$-ray irradiation in the presence of ethyl 2-(ethoxycarbonothioylthio) acetate (Scheme 15). However, no information (e.g. deeper NMR analysis) was provided to ensure which of units was adjacent to the xanthate moiety. Such synthesized poly(CTFE or HFP-alt-BVE) copolymers were used as macroCTAs for the subsequent chain extension of VAc, leading to poly(CTFE or HFP-alt-BVE)- $b$ poly(VAc) diblock copolymers.[125, 126]

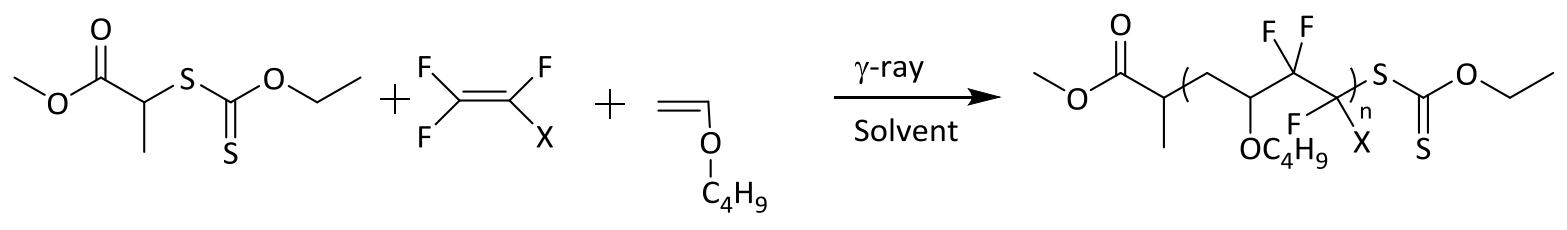

Scheme 15. MADIX alternated copolymerizations of CTFE (for $\mathrm{X}=\mathrm{Cl}$ )[125] or HFP (for $\mathrm{X}=$ $\left.\mathrm{CF}_{3}\right)[126]$ with n-butyl vinyl ether (BVE) initiated under ${ }^{60} \mathrm{Co} \gamma$-ray irradiation in the presence of ethyl 2-(ethoxycarbonothioylthio) acetate.

The same team[126] modified xanthate end-group in the poly(HFP-alt-BVE) copolymer with a sulfonic acid function by oxidation (Scheme 16). Advantageously, this newly developed method occurs under mild sulfonation conditions. 


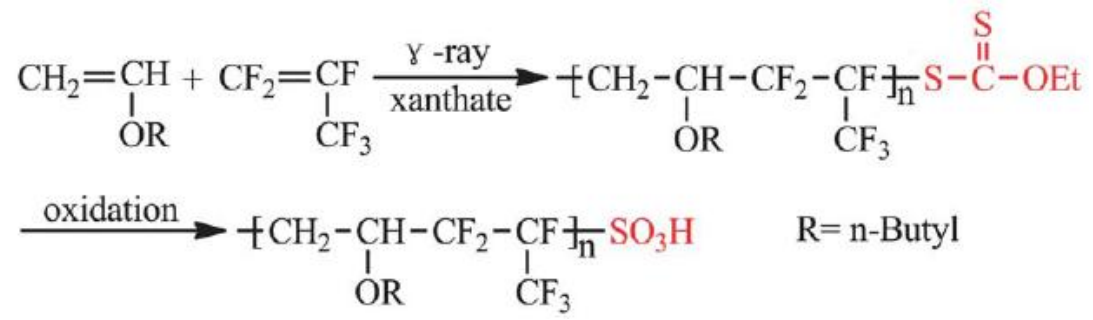

Scheme 16. Radical copolymerization of HFP and BVE and the modification reaction of the xanthate end group into sulfonic acid.[126] Reproduced with permission from Royal Society of Chemistry.

\subsubsection{RAFT copolymerization of tetrafluoroethylene with isobutyl vinyl ether}

Puts et al.[127] reported the unprecedented and only RAFT/MADIX copolymerization of tetrafluoroethylene (TFE) with isobutyl vinyl ether using the same xanthate as above initiated by benzoyl peroxide. This strategy was compared to that attempted under conventional copolymerization (i.e. without any xanthate) and in all cases alternated poly(TFE-alt-iBuVE) copolymers were obtained. Degrees of polymerization $c a$. 20 were targeted and such copolymers (Figure 7) were well-characterized by ${ }^{1} \mathrm{H},{ }^{19} \mathrm{~F},{ }^{1} \mathrm{H}^{-19} \mathrm{~F}$ heteroCOSY NMR and MALDI-TOF MS spectroscopy and SEC.

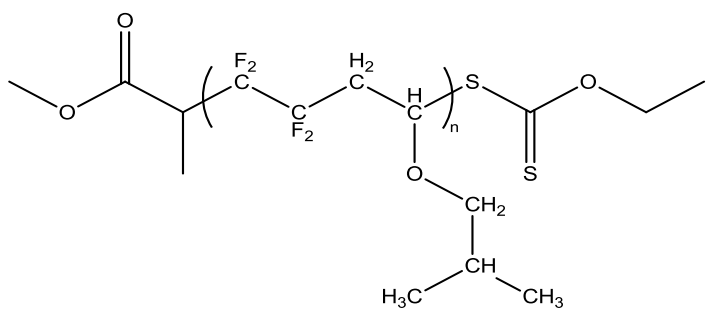

Figure 7. Structure of poly(TFE-alt-iBuVE) alternating copolymer from the RAFT copolymerization of TFE and iBuVE initiated by benzoyl radical and controlled by O-ethyl-S-(1methyloxycarbonyl)ethyl xanthate.[127] 
Their molar masses ranged from 1200 to $2000 \mathrm{~g} \cdot \mathrm{mol}^{-1}$ at $15 \mathrm{~min}$ to $24 \mathrm{hrs}$, respectively, while narrow $\oslash$ s (1.08 and 1.11, respectively) were noted. The chain-end ratios were nearly time invariant, with a slight tendency to accumulate TFE chain ends over time. Proton transfer from DMC to the macroradicals was observed in both the conventional and RAFT polymerizations while a linear Mn vs monomer conversion relationship (Figure 8) was established.

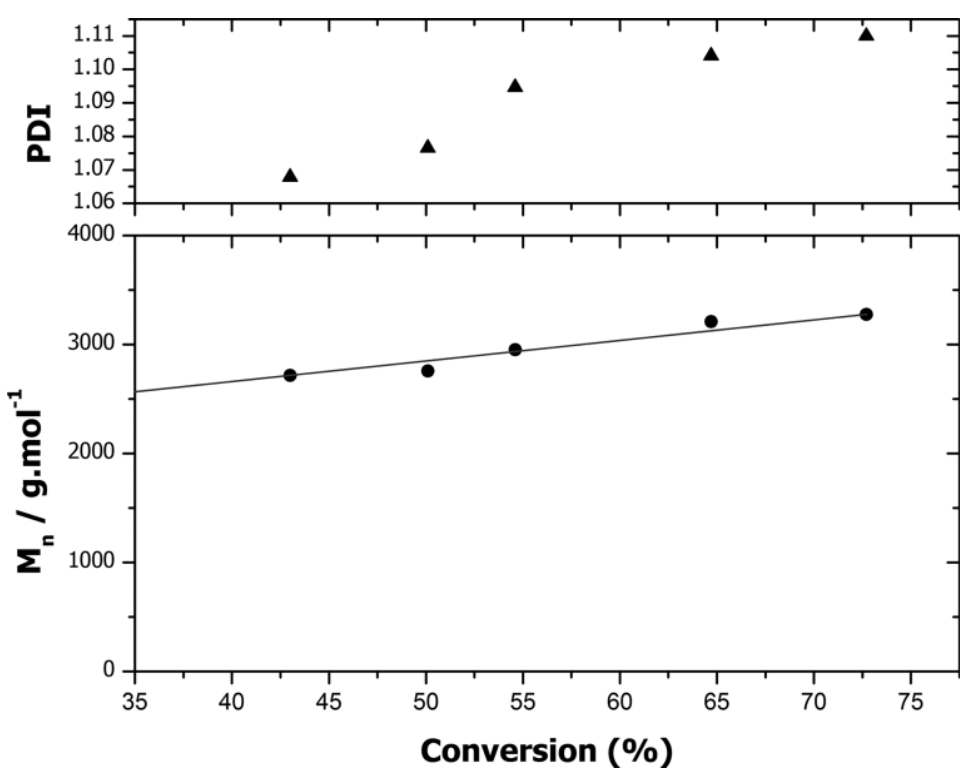

Figure 8. Evolution of $M_{n}$ (from GPC) and $\oslash$ as a function of monomer conversion for the MADIX copolymerization of TFE and iBuVE using a 1:1 ratio of monomers and an initial $[\text { Monomers }]_{0}:[\mathrm{CTA}]_{0}:[\mathrm{BPO}]_{0}$ molar ratio of 20:1:0.1, with O-ethyl-S-(1-methoxycarbonyl)ethyldithiocarbonate as CTA.[127] Reproduced with permission from American Chemical Society.

\subsubsection{Conclusion}

More recently, RAFT has been used as an interesting technique able to control various copolymerizations of VDF (with TrFE, TFP and PMVE) and CTFE, HFP and TFE with vinyl ethers. Most of these studies also led to block copolymers (section 5.2). These reactions 
occurred in solution and obviously led to low molar mass copolymers.

\subsection{Synthesis of fluorinated copolymers using boranes}

Chung's team[50, 93] reported PVDF-based co- and terpolymers of VDF and TrFE or CTFE to tune the electroactive properties of the resulting copolymers for applications as electroactive devices.

These authors produced original poly(VDF-co-CTFE) copolymers and poly(VDF-ter-CTFE-ter$\mathrm{F}_{2} \mathrm{C}=\mathrm{CF}-\mathrm{R}-\mathrm{Si}(\mathrm{OR})_{2} \mathrm{CH}_{3}$ ) terpolymers (Scheme 17) obtained in solution or in bulk at ambient temperature, in reasonable yield. The alkoxy silane function favored a better adhesion onto glass.

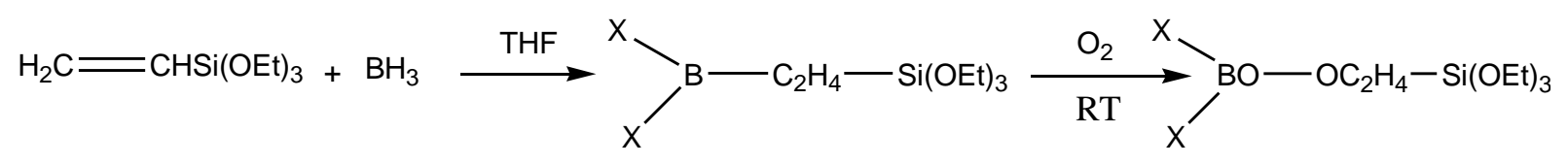

A

A $\underset{\mathrm{F}_{2} \mathrm{C}=\mathrm{CFC}{ }_{2} \mathrm{H}_{4} \mathrm{SiOR}\left(\mathrm{CH}_{3}\right)_{2}}{\stackrel{\mathrm{H}_{2} \mathrm{C}=\mathrm{CF}_{2} / \mathrm{F}_{2} \mathrm{C}=\mathrm{CFCl}}{\longrightarrow}}$

RT

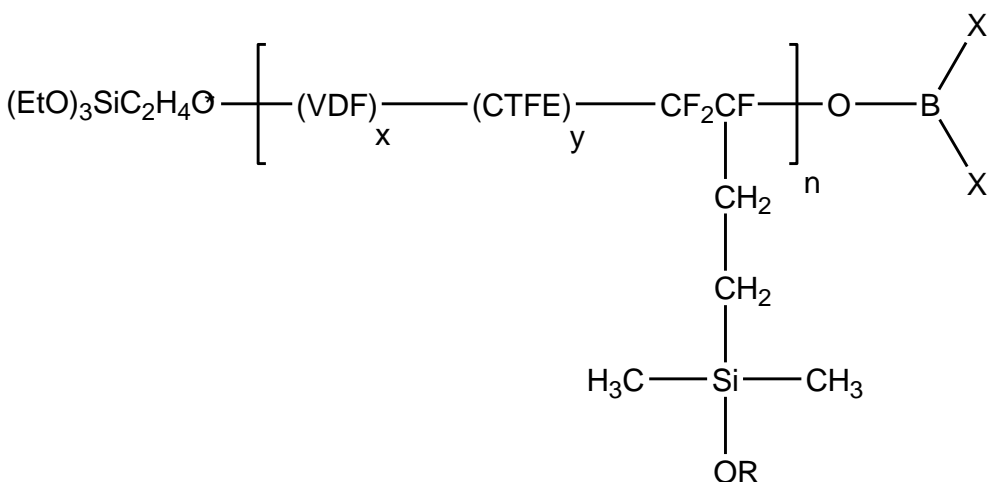

where $\mathrm{X}$ can be $\mathrm{H}$ or $\mathrm{C}_{2} \mathrm{H}_{4} \mathrm{Si}(\mathrm{OEt})_{3}$.

Scheme 17. Synthesis of original poly(VDF-ter-CTFE-ter- $\mathrm{CF}_{2} \mathrm{CF}_{\mathrm{R}} \mathrm{Si}(\mathrm{OR})_{3}$ terpolymers by borane mediated terpolymerization of VDF, CTFE and a functional silane.[50] Reproduced with permission from Wiley.

4.4. Synthesis of fluorinated copolymers via OMRP and other strategies

4.4.1 Copolymerization of VAc with $1 \mathrm{H}, 1 \mathrm{H}, 2 \mathrm{H}, 2 \mathrm{H}$-perfluorohexylethylene 
Demarteau et al.[128] reported the controlled copolymerization of $1 \mathrm{H}, 1 \mathrm{H}, 2 \mathrm{H}, 2 \mathrm{H}-$ perfluorohexylethylene (PFHE) and VAc to prepare well-defined statistical poly(PFHE-statVAc) copolymers of different compositions via cobalt-mediated radical polymerization (CMRP) at $40{ }^{\circ} \mathrm{C}$ starting from an alkylcobalt(III) initiator (Scheme 18). Reactivity ratios of the comonomers were determined to be $r_{V A c}=0.18$ and $r_{P F H E}=0$ at $40{ }^{\circ} \mathrm{C}$, which suggested a tendency towards alternating copolymerization. Furthermore, the authors prepared unprecedented PFHE/VAc containing block copolymers both via a single-step approach or through sequential copolymerizations. Especially, an increasing PFHE amount induced a negative signal in SEC using a refractive index detector. Finally, hydrolysis of the pendant ester groups in PVAc blocks under mild conditions led to the corresponding fluorinated copolymers bearing oligo(vinyl alcohol) (VOH) (Scheme 18) as new amphiphilic copolymers, the properties of which were studied by the authors.

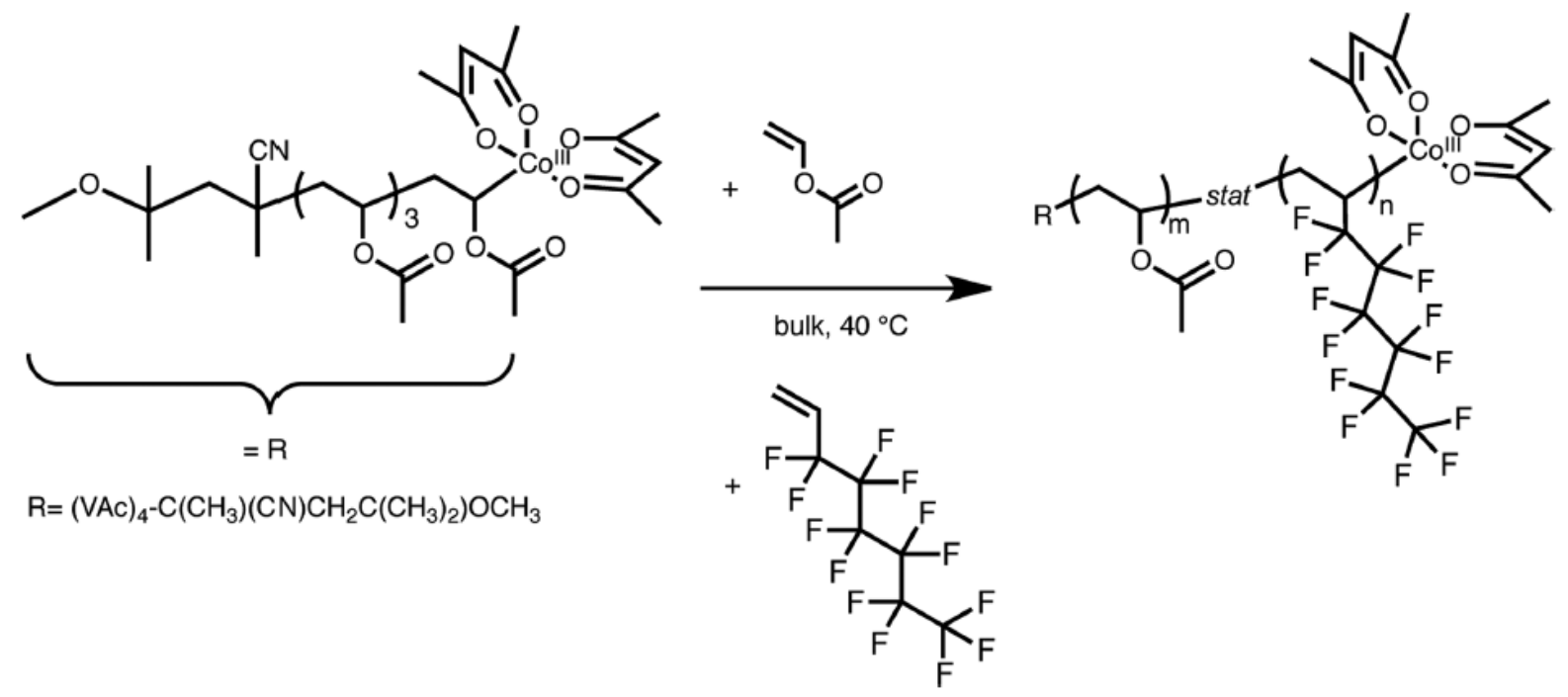

Scheme 18. Synthetic Route for the Copolymerization of Perfluorohexylethylene (PFHE) with Vinyl Acetate (VAc).[128] Reproduced with permission from American Chemical Society.

Self-aggregation study of the $\mathrm{PVAc}_{57}-b$-poly(PFHE-stat-VAc) $)_{95}$ and $\mathrm{PVOH}_{57}-b$-poly(PFHE-stat- 
$\mathrm{VOH})_{95}$ copolymers revealed the formation of spherical nanoobjects with a mean diameter of 31 nm (Figure 9, 2a, white arrow), as noted by TEM, that were confirmed by DLS (the number distribution for these diameters were $25-45 \mathrm{~nm}$ ).

1. PVAc-b-poly(PFHE-stat-VAc)

a)

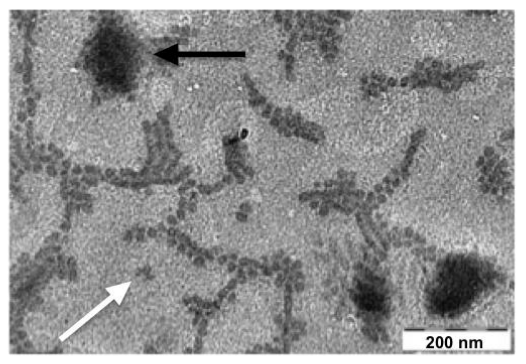

2. PVOH-b-poly(PFHE-stat-VOH)

a)

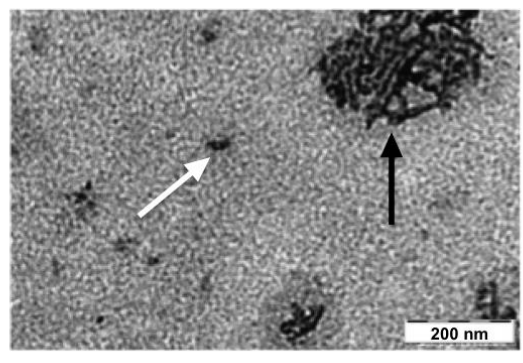

b)

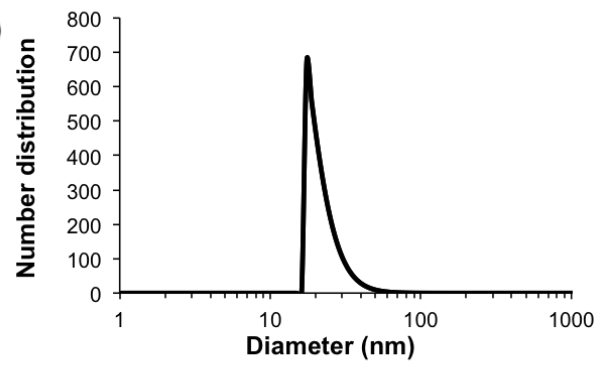

b)

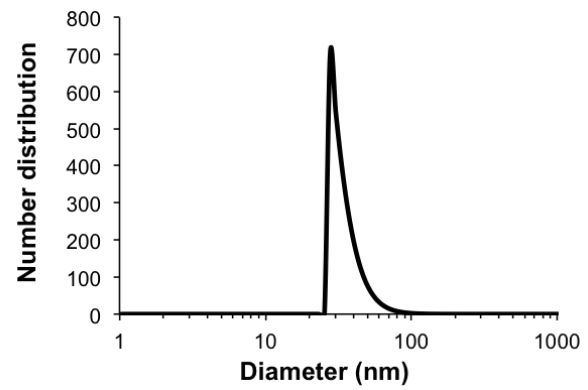

Figure 9. TEM images and number distribution over the diameter (calculated from DLS analysis) of micelles synthesized from PVAc- $b$-poly(PFHE-stat-VAc) copolymers in methanol and the corresponding hydrolyzed BCP in water. On both TEM images, white arrows indicate spherical particles while black ones show aggregates.[128] Reproduced with permission from American Chemical Society.

\subsubsection{Copolymerization of VAc with CTFE}

Conventional radical copolymerization of VAc and CTFE is well known.[129-131] Thomas and O'Shaughnessy[129] reported a detailed kinetic study of the (co)polymerization of CTFE in bulk and in several solvents. The emulsion copolymerization of CTFE, VAc and vinyl propionate by redox initiators was achieved by Murray et al.,[130] while Baradie and Shoichet[131] performed 
such a conventional copolymerization in $\mathrm{scCO}_{2}$. The results revealed that, whatever the process in the medium, VAc was more reactive than CTFE. In contrast, Wang et al.[132] reported the cobalt mediated radical copolymerization of CTFE with VAc initiated by AIBN in the presence of $\mathrm{Co}(\mathrm{acac})_{2}$ as controlling agent. Both the molar masses and molar compositions of the copolymers could be "controlled", however the $Ð$ values of the resulting copolymers were high. This is probably due to the fact that the resulting poly(VAc-co-CTFE) copolymers were not quenched by TEMPO to avoid recombination.[49] Surprisingly, a higher CTFE reactivity was observed in contrast to that obtained from the conventional radical copolymerization.[129-131] Furthermore, chain extension polymerization of such obtained fluorinated copolymers was also achieved. One major issue is the absence of CTFE and VAc contents in these copolymers (simply achieved either by NMR with a standard or elemental analysis by chlorine \%). In addition, the molar masses were quite high $\left(>100 \mathrm{~kg} \cdot \mathrm{mol}^{-1}\right)$ and do not fit with the theoretical ones calculated from \%C. Furthermore, it is known that polymer terminated by $\mathrm{Co}(\mathrm{acac})_{2}$ must be quenched by TEMPO (or a mercaptan or $\mathrm{SnBu}_{3} \mathrm{H}$ ) to be stable under air.[133, 134] In addition, the dispersities were rather high (1.49-1.57) and the authors did not mention the nature of end-groups (CTFE-Co(acac $)_{2}$ or VAc-Co(acac $\left.)_{2}\right)$.

4.5 Synthesis of fluorinated copolymers via ATRP

Though ATRP of acrylates bearing fluorinated groups was successful,[57] to the best of our knowledge, no ATRP of fluoroalkenes has been reported probably because of the strong $\mathrm{CF}_{2}-\mathrm{X}$ bond (X being a chlorine or bromine atom). However, "dendritic" copolymers, soluble in common organic solvents, based on CTFE and p-chloromethylstyrene (CMS) were surprisingly produced in the presence of copper(I) bromide-bipyridine (bpy), probably from the C-Cl bond of both CTFE and CMS (Scheme 19).[135] 


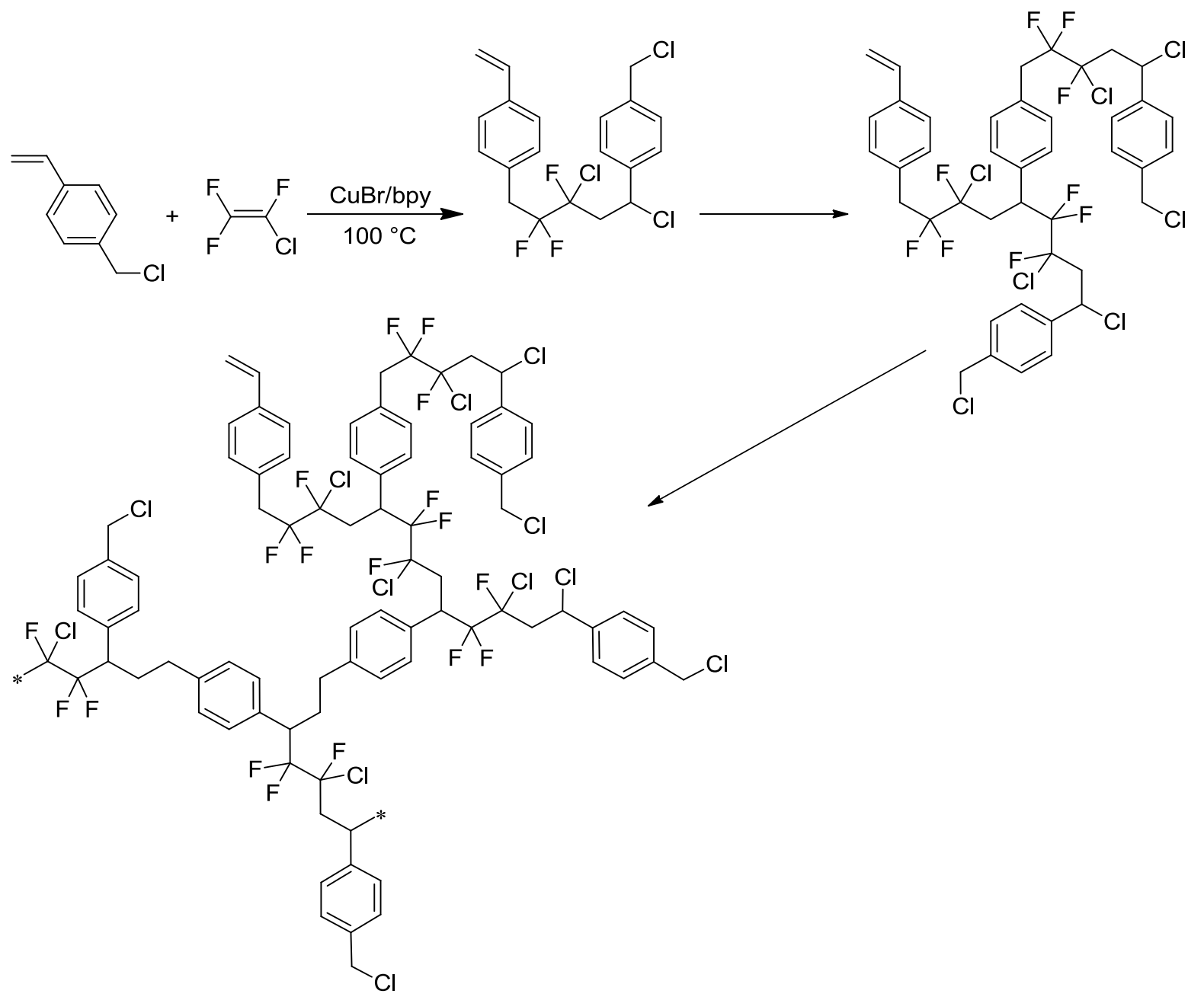

Scheme 19. Synthesis of dendritic copolymers based on CTFE with $p$-chloromethylstyrene (CMS) in the presence of copper(I)bromine-bipyridine (bpy) complex via ATRP of CMS with CTFE.[135] Reproduced with permission from Wiley.

\subsection{Conclusion}

RDR copolymerization of fluorinated monomers has led to many academic and industrial research with the success to be industrially implemented for ITP. Borane mediated copolymerizations and ATRP are rare. Based on the above discussion, it can be concluded that RAFT polymerization has emerged as the most versatile technique for the copolymerization of various fluorinated alkenes including VDF, CTFE and TFE. RAFT can tolerate a wide range of 
monomers bearing diverse functionalities and does not require stringent reaction conditions. Thus, it has the potential to emerge as one of the most versatile RDRP techniques in the future.

\section{Synthesis of fluorinated block copolymers containing VDF and other monomers via RDRP}

Block copolymers (BCPs) are composed of two (or more) chemically different polymer chains linked to each other. This connectivity prevents from macrophase separation but enables microphase separation at the nanoscale. The microphase separation can be tuned from several factors as the total degree of polymerization $(\mathrm{N})$, the architecture, the volume fractions, and the Flory-Higgins parameter $(\chi) .[136]$ Changing the block ratios yields to different morphologies: lamellar, spheres, hexagonally packed cylinders, and bicontinuous gyroid phase.[137] The addition of a third polymer can bring to even more complex structures like a star or branched polymers.[138] Different polymerization techniques have been developed to enable synthesis of fluorinated copolymers. Extensive research has been carried out in the last decade in order to develop these methods, as demonstrated below:

\subsection{Synthesis of VDF-based copolymers via ITP}

Several researchers have reported the synthesis of fluorinated block copolymers and the most attractive reviews displaying many strategies were published by Loos'[139] and Asandei's[140] groups.

5.1.1 Synthesis of VDF-based block copolymers using thermal initiators In 1970s, Tatemoto first reported ITP.[31-33] Subsequent to this discovery, Otsu's team developed the initiation-transfer-termination method,[40, 41] though that technique was not applied to fluorinated monomers. Valade et. al.[141] reported the synthesis of PVDF- $b$-PSt 
block copolymers by ITP. The authors also investigated detailed kinetics and mechanistic study of the reaction. Then, Asandei[140] reviewed various strategies of BCPs via photo ITP in the presence of $\mathrm{Mn}_{2}(\mathrm{CO})_{10}$. In addition, for another application (electroactive materials) Ameduri and Allaeddine synthesized block copolymers via the controlled radical copolymerization of trifluoroethylene (TrFE) with another fluoromonomer, such as VDF or 2,3,3,3-tetrafluoroprop-1-ene (1234yf), in the presence of 1-iodo-PVDFmacroCTA (Scheme 20).[124]

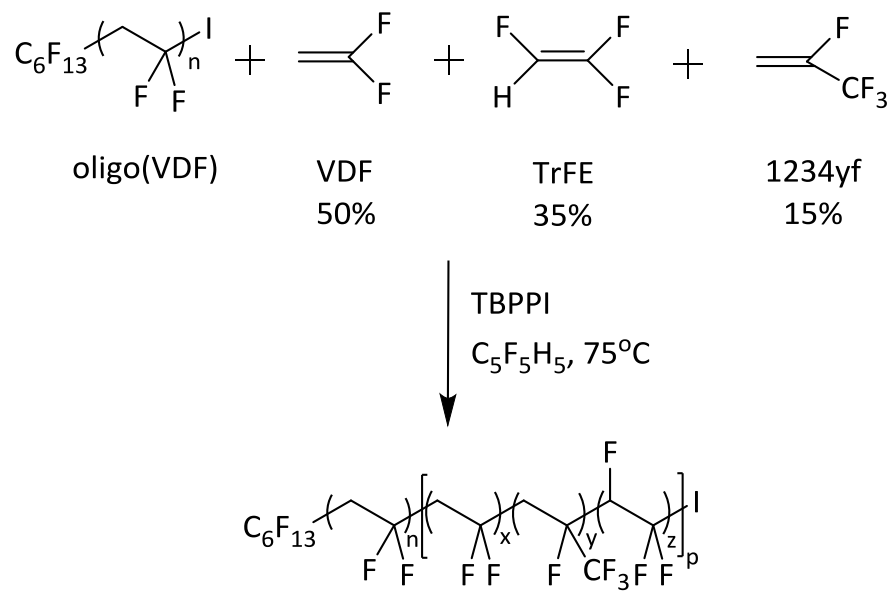

Scheme 20. Synthesis of PVDF-b-poly(VDF-ter-TrFE-ter-1234yf) block copolymers by ITP, where 1234yf and TBPPI stand for 2,3,3,3-tetrafluoroprop-1-ene and tert-butylperoxypivalate, respectively.[124]

Recently, Lopez and co-workers first synthesized various fluoroiodinated macroCTA based on VDF, CTFE, and vinylidene dichloride (VDC) before producing block copolymers by ITP (Scheme 21).[142, 143] 


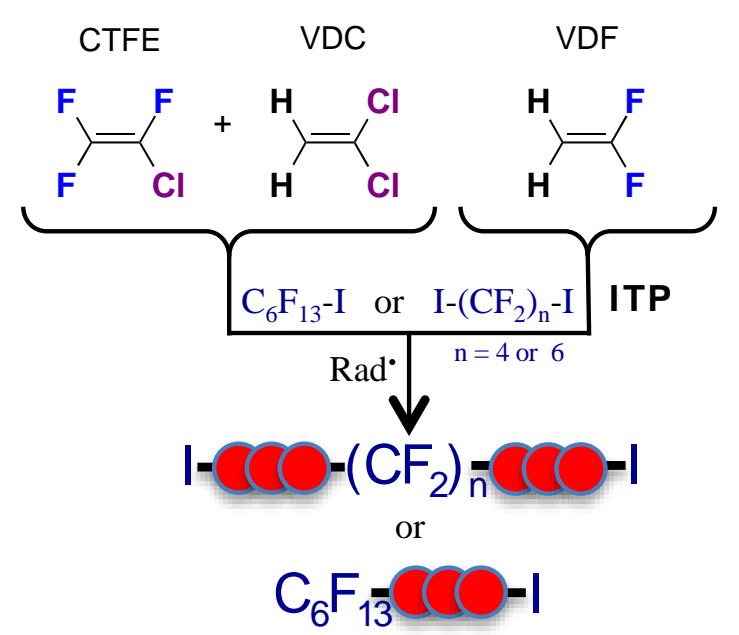

Scheme 21. Synthesis of fluorinated macroCTA by ITP of various halogenated monomers where VDC is vinylidene chloride.[142] Reproduced with permission from American Chemical Society.

Monomodal GPC traces and a clear shift with respect to that of the macro-CTA were observed indicating the successful formation of block copolymer (Figure 10).

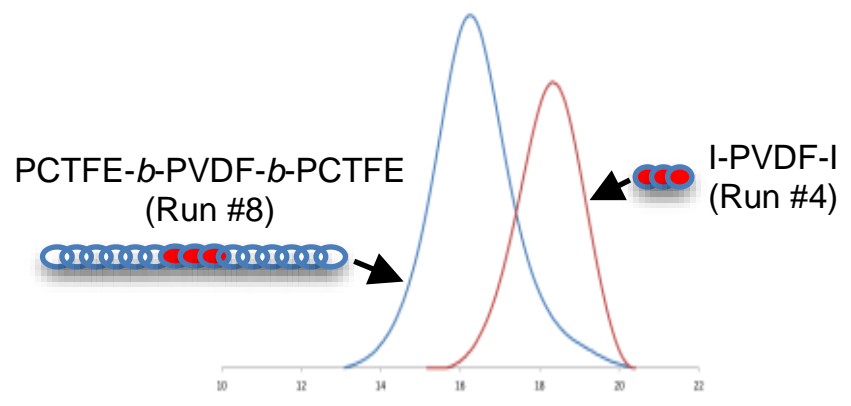

Figure 10. GPC traces for telechelic diiodo PVDFmacro-CTA (Run \#4) and PCTFE- $b$-PVDF- $b$ PCTFE triblock copolymer (Run \#8) (in DMF using PMMA standards).[142] Reproduced with permission from American Chemical Society.

Following the strategy to get both PVDF and PS sequences in a block copolymer, Recently, Zapsas et al. [144] synthesized copolymers consisting of crystalline polymethylene (PM) and crystalline PVDF blocks as well as triblock terpolymers including polystyrene (PS) as the central block. Two/three different polymerization methods were used for these syntheses such as 
polyhomologation, ATRP and ITP along with chain-end postpolymerization reactions (Scheme 22).
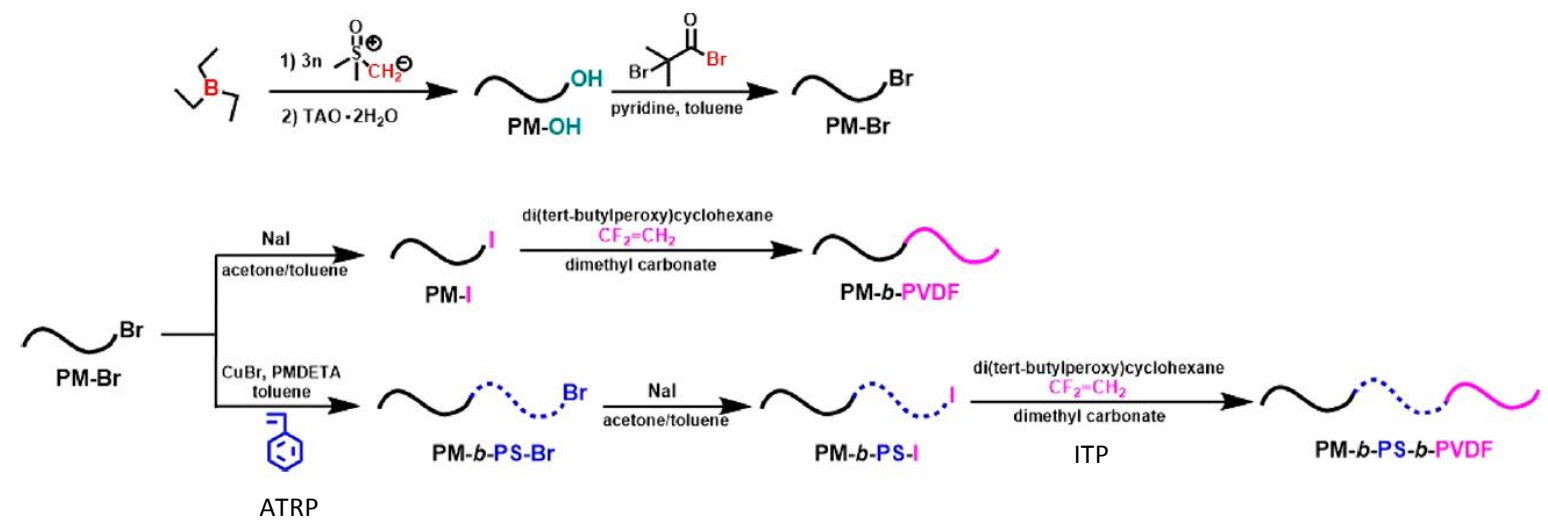

Scheme 22. Synthesis of polymethylene- $b$-PVDF (PM- $b$-PVDF) diblock and PM- $b$-PS- $b$-PVDF triblock terpolymer by subsequent polyhomologation and ITP or polyhomologation, ATRP and ITP techniques, respectively.[144] Reproduced with permission from American Chemical Society.

Historically and, by far, the deepest studies that led to commercial materials were developed by Tatemoto and co-workers[31, 38] who successfully synthesized Hard- $b$-Soft- $b$-Hard triblock copolymers via the ITP of VDF and HFP using $\alpha, \omega$-diiodoperfluoroalkanes $\left(\mathrm{IC}_{4} \mathrm{~F}_{8} \mathrm{I}\right.$ or $\left.\mathrm{IC}_{6} \mathrm{~F}_{12} \mathrm{I}\right)$ as the CTAs. Subsequently, they chain extended the resulting telechelic diiodopoly(VDF-co-HFP) copolymer (as a soft segment) with VDF (or ethylene and TFE) (Scheme 23) producing Hard- $b$-Soft- $b$-Hard thermoplastic elastomers (TPEs). These TPEs had potential applications in aeronautics or automotive industries as pressure-sensitive adhesives, hot melts, O-rings, tough transparent films and sealants.[2-8, 31-33] In the mid-80ies, Daikin Company marketed such synthesized TPE (which are composed of central soft segments, e.g., low glass transition temperature $\left(T_{g}\right)$ blocks, containing the poly(VDF-co-HFP) as described 
above or composed of poly(VDF-co-CTFE), poly(VDF-ter-HFP-ter-TFE), poly[ethylene-terPMVE-ter-TFE] elastomeric blocks), under the Dai-el® trademark. Hard blocks of these TPEs were composed of various crystalline polymeric sequences [PTFE, or poly(ethylene-altTFE) or poly(ethylene-ter-TFE-ter-HFP) in the case of Dai-el@ T530][31-33, 65, 66, 145] or PVDF in the case of Dai-el® T-630.[31, 32, 38, 145]

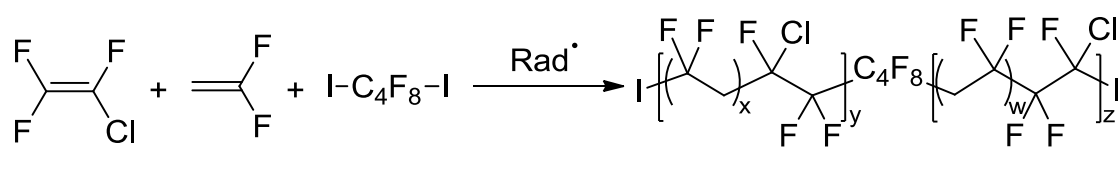

I-Dolv(VDF-Co-CTFE)-I

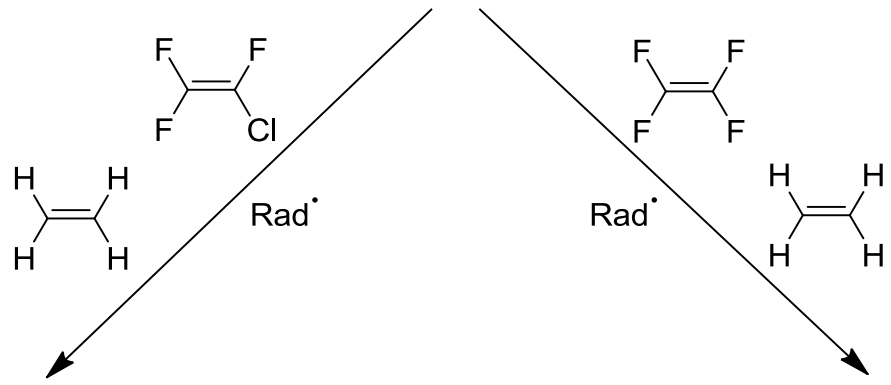

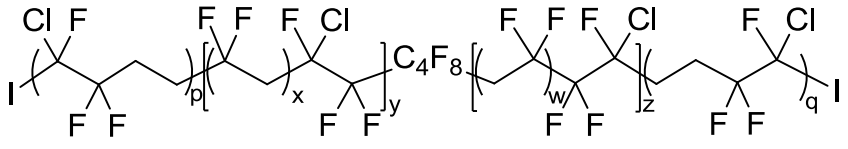

l-polv(E-alt-CTFE)-b-polv(VDF-co-CTFE)-b-polv(E-alt-CTFE)-I

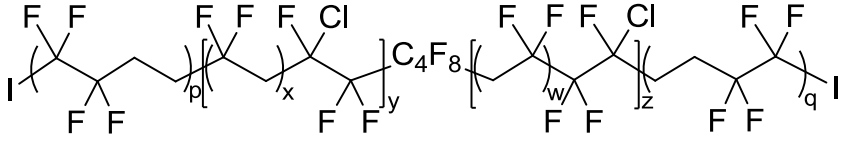

l-polv(E-alt-TFE)-b-polv(VDF-co-CTFE)-b-polv(E-alt-TFE)-I

Scheme 23. Synthesis of triblock TPE using telechelic diiodo poly(CTFE-co-VDF) copolymers as CTA.[31-33, 65, 66, 145] Reproduced with permission from American Chemical Society.

Non-homopolymerizable perfluoroalkyl vinyl ethers (PAVE) $\left(r_{P A V E}=0\right)$ can be easily copolymerized with either TFE or VDF to yield random copolymers which are semicrystalline (with reduced crystallinity compared with those of PTFE and PVDF) or amorphous.[146, 147] The key challenge of these polymers aims at producing materials with $T_{g}$ lower than $-40{ }^{\circ} \mathrm{C}$ for potential low temperature applications,[148] along with good thermal stability for future 
applications in aeronautics and automotive industries or any device that requires to keep its properties at low temperatures.

\subsubsection{Synthesis of VDF-based block copolymers using photo initiators}

As detailed in Section 3.1.2, Asandei et al.[79, 80] obtained VDF based copolymer using photomediated ITP. This team also disclosed the effect of a series of transition metals and ligands for the synthesis of well-defined PVDF block copolymers by quantitative activation of both $\mathrm{PVDF}-\mathrm{CH}_{2}-\mathrm{CF}_{2}-\mathrm{I}$ and $\mathrm{PVDF}-\mathrm{CF}_{2}-\mathrm{CH}_{2}-\mathrm{I}$ chain ends.[81]

Table 1 Synthesis of PVDF- $b$-poly(M) or poly(M)- $b$-PVDF- $b$-poly(M) di/tri-block copolymers and dependence of $\mathrm{Mn}$ and $\mathrm{Mw} / \mathrm{Mn}$ on conversion in $\mathrm{Mn}_{2}(\mathrm{CO})_{10}$-photomediated hydrocarbon M monomer from PVDF-I or I-PVDF-I.[79]

\begin{tabular}{|c|c|c|c|c|c|c|c|}
\hline \multirow[t]{2}{*}{ Monomer (M) } & \multicolumn{2}{|c|}{ PVDFI or I-PVDF-I } & \multirow[t]{2}{*}[\mathrm{M}]{$/[\mathrm{PVDF}] /\left[\mathrm{Mn}_{2}(\mathrm{CO})_{10}\right]$} & \multirow[t]{2}{*}{ Conv. (\%) } & \multirow[t]{2}{*}{$M / V D F$} & \multirow[t]{2}{*}{$M_{n}$} & \multirow[t]{2}{*}{$\oplus$} \\
\hline & $M_{n}$ & $\boldsymbol{\theta}$ & & & & & \\
\hline Styrene & 2,500 & 1.34 & $60 / 1 / 2$ & 67 & $70 / 30$ & 14,500 & 2.25 \\
\hline Styrene & 11,500 & 1.48 & $4,000 / 1 / 5$ & 12 & $92 / 8$ & 44,700 & 1.92 \\
\hline Butadiene & 1,400 & 1.48 & $200 / 1 / 1$ & 25 & $62 / 38$ & 4,700 & 2.00 \\
\hline Vinyl Chloride & 1,800 & 1.29 & $100 / 1 / 1$ & 35 & $77 / 23$ & 20,100 & 1.52 \\
\hline Vinyl Acetate & 1,500 & 1.49 & $100 / 1 / 0.2$ & 30 & $65 / 35$ & 11,000 & 1.70 \\
\hline Methyl Acrylate & 2,300 & 1.52 & $75 / 1 / 4$ & 40 & $72 / 28$ & 9,000 & 2.46 \\
\hline Acrylonitrile & 2,100 & 1.31 & $50 / 1 / 1$ & 25 & $74 / 26$ & 25,800 & 2.33 \\
\hline
\end{tabular}

The same group also employed this $\mathrm{Mn}_{2}(\mathrm{CO})_{10}$-system to prepare well-defined sulfonated and fluorinated block copolymers for application in fuel cell membranes via sequential copolymerization of neopentyl styrene sulfonate and VDF.[149] They further used hypervalent iodide carboxylate, $\left(\mathrm{CX}_{3} \mathrm{CO}_{2}\right)_{2} \mathrm{Ph}$ mediated metal-free protocol for the synthesis of PVDF- $b$-poly(M) block copolymers[81] [where 'M' stands for styrene (S), butadiene, 
2,2,2-trifluoroethyl methacrylate, and surprisingly methyl-2-(trifluoromethyl) acrylate] (known not to homopolymerize) $[150,151]$ in conjunction with external $\left[\mathrm{I}_{\left.\left(\mathrm{CF}_{2}\right)_{6} \mathrm{I}\right]}\right.$ and/or in situ generated $\left(\mathrm{CF}_{3} \mathrm{I}\right)$ iodinated CTA.[80] Then, the same group extensively studied a series of metal carbonyls in the ITP of VDF and block copolymerization of VDF thereof.[81] However, in spite of the initial excitements on this photomediated ITP, these systems remain of academic interest only and cannot be scaled up for commercial applications because the major drawback of this procedure stems from the hazardous high pressures at which photochemical reactors may not withstand.

Golzari et al.[152] reported the synthesis of PVDF- $b$-PS and PVDF- $b$-PMMA block copolymer starting from PVDF-I macroinitiator mediated by $\mathrm{Mn}_{2}(\mathrm{CO})_{10}$ under UV irradiation. PVDF-I macroinitiator was synthesized by ITP either in supercritical $\mathrm{CO}_{2}[70,71]$ or in emulsion.[77] SEC and NMR spectroscopy clearly revealed that the entire PVDF-I was converted into block copolymers. The crystal phase of such resulting block copolymers was identified using XRD, FT-IR, and differential scanning calorimetry (DSC) analyses. The two former techniques suggested transformation of $\alpha$ crystalline phase into $\beta$ crystalline one of PVDF in the block copolymers (Figure 11), while AFM analyses displayed a phase separation in the resulting block copolymers. 


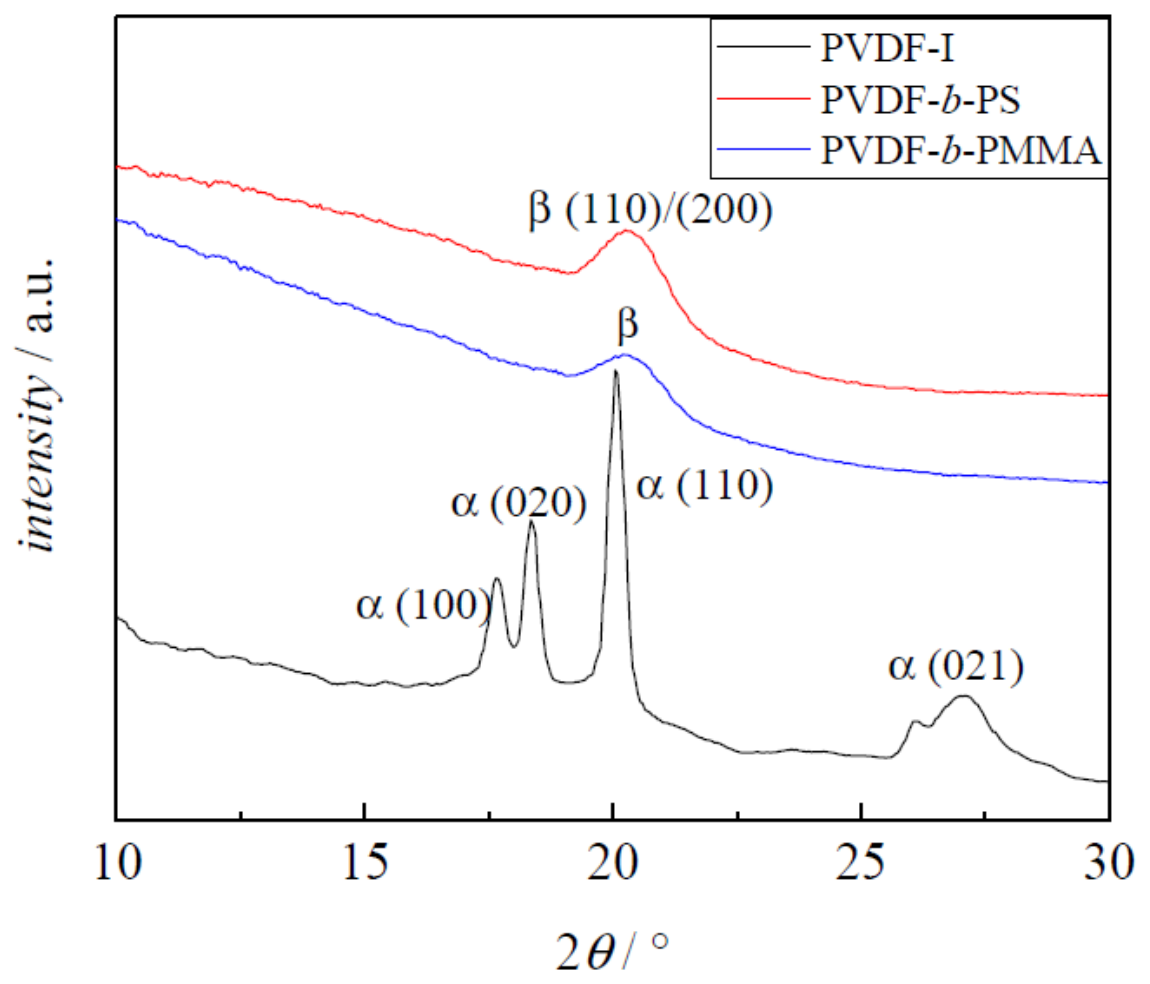

Figure 11. XRD spectra of PVDF-I macroinitiator (black), block copolymers with MMA (blue) and with styrene (red).[152] Reproduced with permission from MDPI.

The same group[77] subsequently reported kinetic Monte Carlo simulation based on detailed mechanistic and kinetic investigation of the transfer processes in semi-batch ITP of VDF under emulsion polymerization condition.

\subsection{Block copolymers via RAFT/MADIX polymerization of fluoroalkenes}

RAFT is a powerful technique to prepare block copolymers.[44-46] From model RAFT polymerization of VDF, a wide range of architectures including BCPs was successfully produced (Scheme 24). 


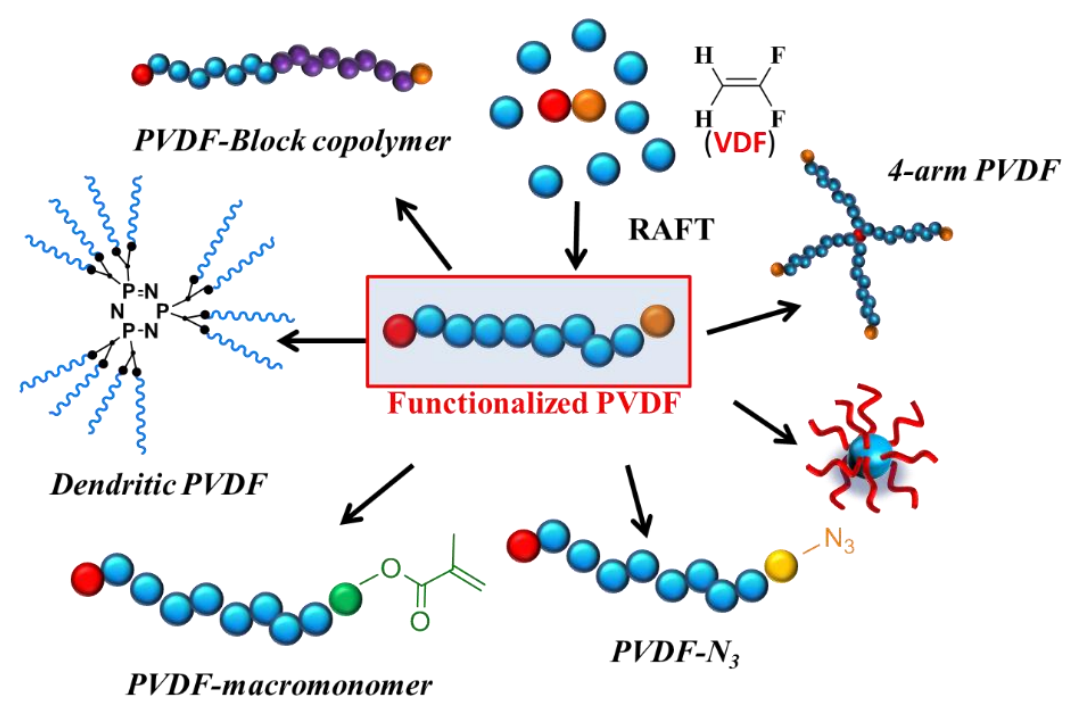

Scheme 24. RAFT polymerization of VDF opening well-designed PVDF architectures.[84-87, 153-155]

MADIX polymerization was subsequently extended to develop polymeric surfactants successfully. Kostov et al.[83] pioneered RAFT copolymerization of VDF and 3.3.3trifluoropropene (TFP). A chain extension of vinyl acetate (Scheme 25) in the presence of the resulting macroCTA, followed by hydrolysis of the PVAc sequence, led to original poly(VDFco-TFP)- $b$-PVOH surfactants.[83] Such amphiphilic copolymers display quasi similar surface tension as that of ammonium perfluorooctanoate (APFO) and with an advantageous possible cleavable/degradable/metabolizable poly(VDF-co-TFP) sequence in contrast to stable perfluorinated moieties in APFO.[156]

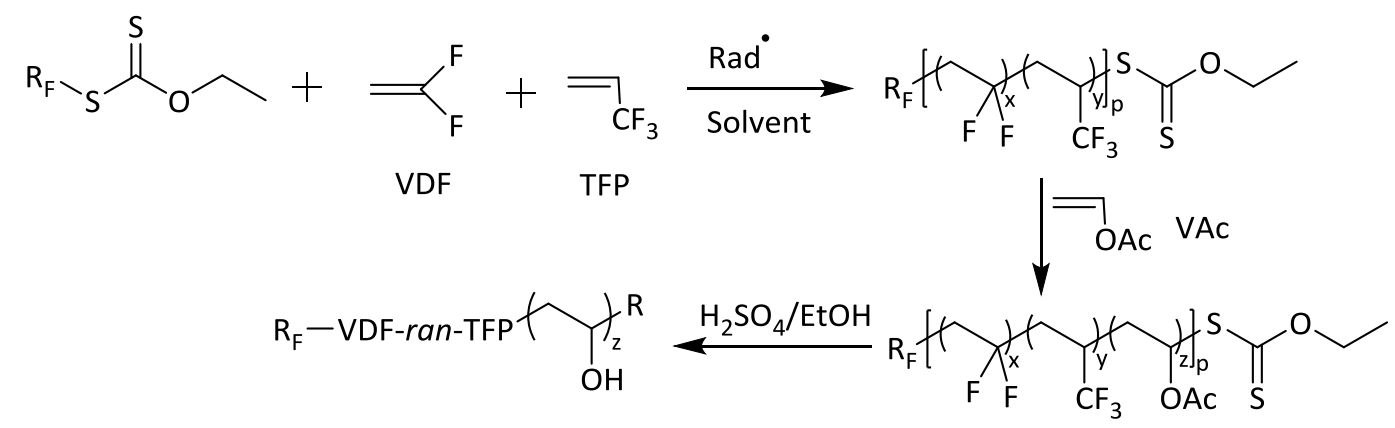


Scheme 25. Sequenced MADIX terpolymerization of VDF, TFP and VAc controlled by a fluorinated xanthate for the synthesis of poly(VDF-co-TFP)-b-oligo(VAc) block copolymers further hydrolyzed into original surfactants.[83] Reproduced with permission from American Chemical Society.

Other surfactants exhibited very interesting $\mathrm{CO}_{2}$-philicity behaviour. One example was given by Destarac's group in the synthesis of polymeric surfactants from the MADIX copolymerization of VDF and PMVE from hydrophilic poly(N,N-dimethylacrylamide) macroRAFT agents (Scheme 26)[157] taking into account that amorphous poly(VDF-co-PMVE) copolymers are $\mathrm{CO}_{2}{ }^{-}$ philic.[158]

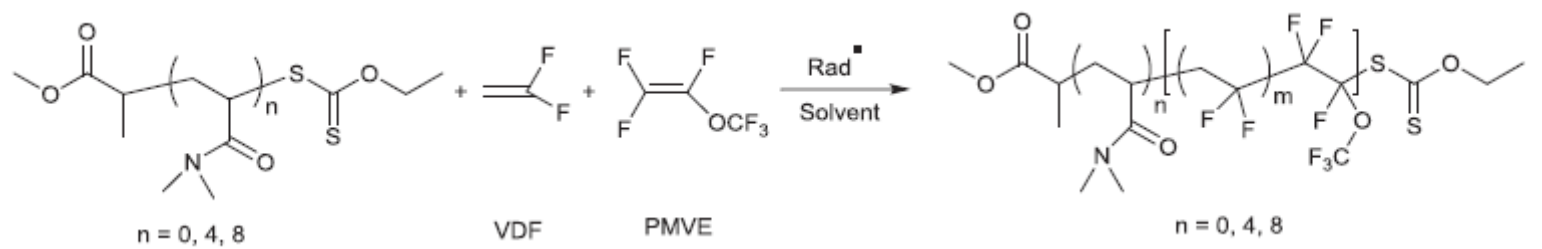

Scheme 26. Synthesis of poly(N,N-dimethylacrylamide) (PDMAc)-b-poly(VDF-co-PMVE) block copolymers by RAFT/MADIX copolymerization of VDF and PMVE from a macroxanthate based on oligo(DMAc) for the synthesis of block copolymers.[157] Reproduced with permission from American Chemical Society.

Wang et al.[126] reported the controlled radical copolymerization of HFP and butyl vinyl ether at room temperature under ${ }^{60} \mathrm{Co} \quad \gamma$-ray irradiation in the presence of ethyl 2(ethoxycarbonothioylthio) acetate (Scheme 15). Such a macroRAFT agent was chain extended by VAc to produce a block copolymer which was clearly identified by SEC (Figure 12). 


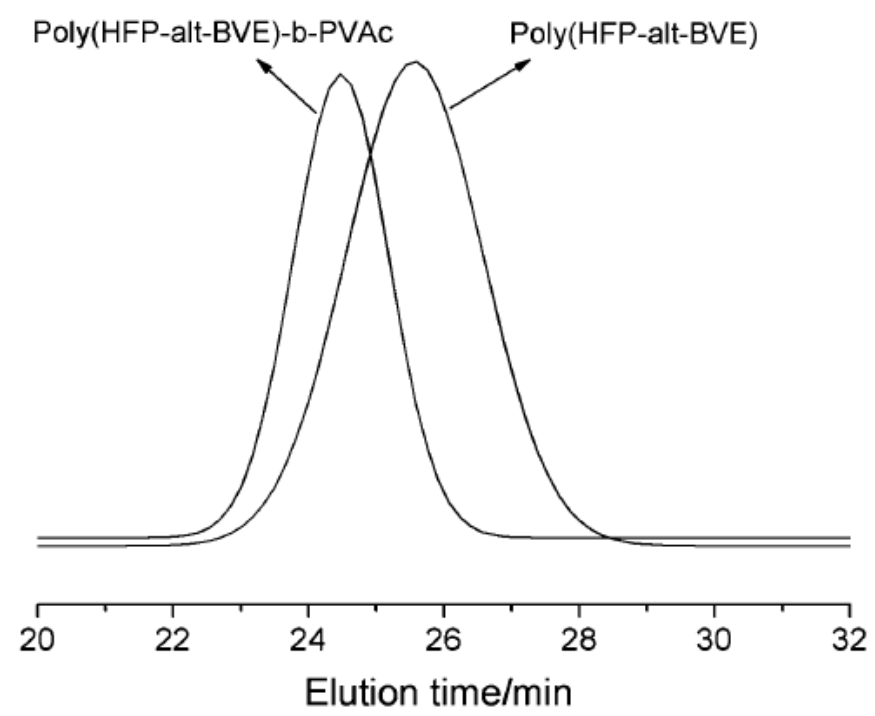

Figure 12. GPC traces of poly(hexafluoropropylene-alt-butyl vinyl ether) \{poly(HFP-alt-BVE)\} (P3) and poly(HFP-alt-BVE)-b-poly(VAc) copolymers.[126] Reproduced with permission from Royal Society of Chemistry.

\subsubsection{Synthesis of VDF-based block copolymers}

Banerjee et al.[154] reported unprecedented synthesis of multiblock PVDF (up to $16300 \mathrm{~g} \mathrm{~mol}^{-1}$ ) with narrow-dispersities $(\Theta=1.26)$ via RAFT polymerization mediated by a trifluoromethyl cyclic xanthate (cy-XA) (Scheme 27). The cy-XA was obtained by cyclothiocarbonation of 1trifluoromethyloxyethylene. The polymerization was controlled as evidenced by the linear increase in the $M_{n}$ vs. VDF conversion. Interestingly, hydrolysis under mild basic conditions enabled the synthesis of $w$-mercapto-PVDF. The as-synthesized multiblock PVDFs were further employed as macroRAFT agents to prepare novel multiblock copolymers using VAc as the second monomer or a mixture of VAc and MAF-TBE which alternate (Scheme 27). As expected, the thermal stability of PVDF was higher than that of PVDF- $b$-PVAc copolymers. PVDF exhibited a melting temperature of $168{ }^{\circ} \mathrm{C}$ and $45 \%$ crystallinity, whereas PVDF- $b$-PVAc and 
PVDF- $b$-poly(VAc-alt-MAF-TBE) block copolymers were amorphous, displaying glass transition temperatures of 41 and $75^{\circ} \mathrm{C}$, respectively.

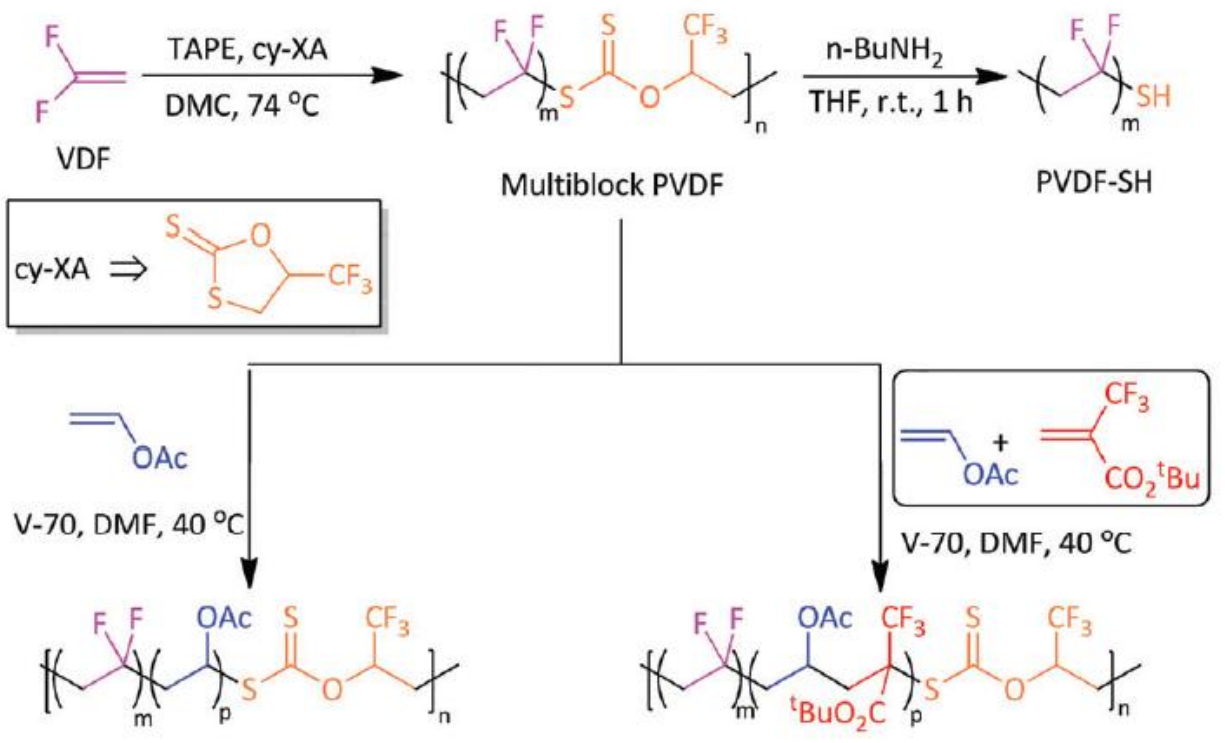

Poly(PVDF- $b$-PVAc) Multiblock

Poly[PVDF-b-poly(VAc-alt-MAF-TBE)] Multiblock

Scheme 27. Synthesis of multiblock copolymers based on VDF, tert-butyl-2-trifluoromethacrylate (MAF-TBE) and VAc controlled by a fluorinated cyclic xanthate (cy-XA) (DMC and TAPE stand for dimethyl carbonate and tert-amyl peroxy-2-ethylhexanoate, respectively).[154] Reproduced with permission from Royal Society of Chemistry.

\subsubsection{Block copolymers based on PVDF and poly(ethylene glycol)}

Folgado et al.[159] reported a novel strategy to synthesize poly(vinylidene fluoride)-blockpoly(ethylene glycol)-block-poly(vinylidene fluoride) (PVDF- $b$-PEG- $b$-PVDF) ABA triblock copolymer from PVDF-Xa $\left(M_{n}=1200 \mathrm{~g} \cdot \mathrm{mol}^{-1} ; Ð=1.32\right.$, prepared by RAFT polymerization as above. Interestingly, this one pot reaction involves the aminolysis of xanthate group functionalities at the chain end, in presence of hexyl amine, followed by the thio Micheal addition click reaction onto a PEG diacrylate $\left(M_{n}=1000 \mathrm{~g} \cdot \mathrm{mol}^{-1}\right.$ and $Ð=1.11$. Such a 
resulting ABA triblock copolymer has a molar mass of $3000 \mathrm{~g} \cdot \mathrm{mol}^{-1}$ and a dispersity of 1.29 . The novel PVDF- $b$-PEG- $b$-PVDF ABC amphiphilic triblock copolymer, prepared by Folgado et al.[159] (Section 4.2.4) exhibited two distinct $T_{g}$ s corresponding to VDF $\left(-34^{\circ} \mathrm{C}\right)$ and PEG (-10 $\left.{ }^{\circ} \mathrm{C}\right)$ segments. This confirms the immiscibility of these two blocks (PVDF and PEG). The degree of crystallinity of the PVDF (47.1\%) and of the PEG $(53.8 \%)$ in the triblock copolymer were also determined from DSC analysis.

\subsubsection{Direct Synthesis of VDF-based amphiphilic diblock copolymers}

PVDF based macroCTAs described in Section 3.1.2 are obviously key intermediates to prepare a wide range of various architectures (Schemes 24 and 28) to allow the chain extension of a second PVAc block by RAFT.

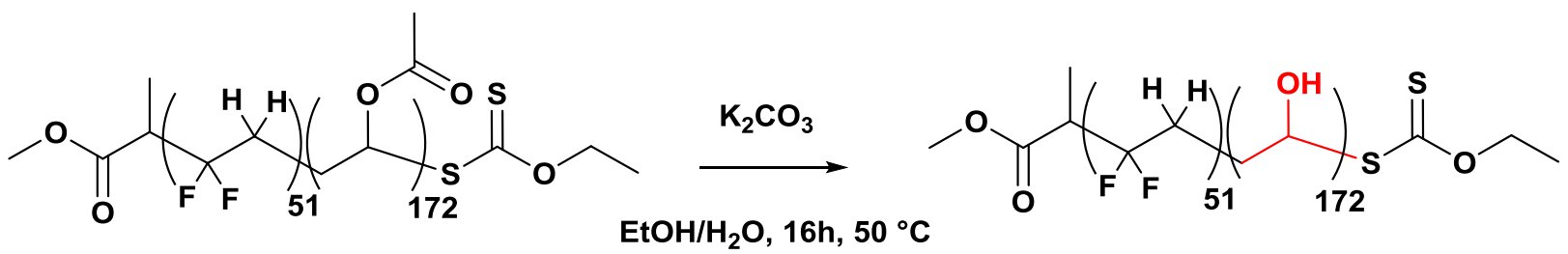

Scheme 28. Hydrolysis of PVAc block into PVOH sequence to prepare PVDF- $b$-PVOH amphiphilic fluorinated block copolymers.[155] Reproduced with permission from Royal Society of Chemistry.

Several examples are given hereafter. Our group attempted the chain extension of various monomers (VAc, N-Vinylpyrrolidine (NVP), butyl acrylate (BA) and dimethylacrylamide) from PVDF-xanthate in RAFT conditions.[153] The reactions were monitored by SEC (Figure 13) and ${ }^{19}$ F NMR spectroscopy to ensure the success of the BCP synthesis. The chain extension of VAc clearly highlighted by vanishing of specific NMR signals and narrow dispersity, was a further 
evidence of the presence of xanthate end-group. Then, hydrolysis of PVAc into PVOH, monitored by both IR and ${ }^{1} \mathrm{H}$ NMR spectroscopies was achieved in mild basic conditions to reduce some dehydrofluorination of PVDF sequence.[155]
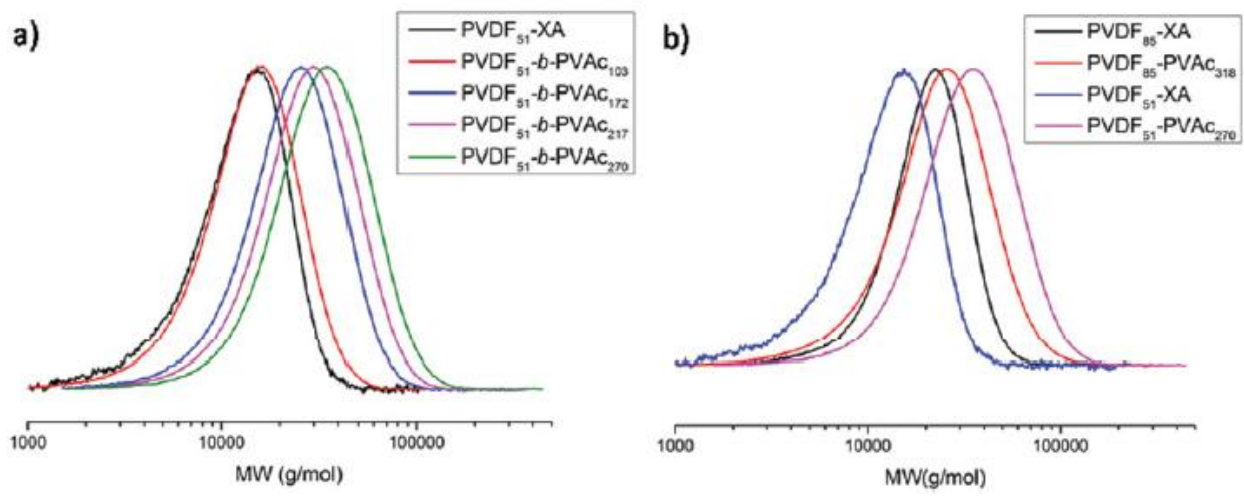

Figure 13. (a) Normalized GPC traces (viscosimetric detector) of $\mathrm{PVDF}_{51}-\mathrm{XA}$ homopolymer, $\mathrm{PVDF}_{51}-b-\mathrm{PVAc}_{103}, \mathrm{PVDF}_{51}-b-\mathrm{PVAc}_{172}, \mathrm{PVDF}_{51}-b-\mathrm{PVAc}_{217}$ and $\mathrm{PVDF}_{51}-b-\mathrm{PVAc}_{270}$ block copolymers. (b) Normalized GPC traces (viscosimetric detector) of $\mathrm{PVDF}_{51}-\mathrm{XA}, \mathrm{PVDF}_{85}-\mathrm{XA}$, $\mathrm{PVDF}_{51}-b-\mathrm{PVAc}_{270}$ and $\mathrm{PVDF}_{85}-b-\mathrm{PVAc}_{318}$ [155] Reproduced with permission from Royal Society of Chemisty.

After purification by dialysis, the amphiphilic BCPs were characterized by cryoTEM and light scattering to observe particle size of ca. $100 \mathrm{~nm} .[155]$

Li et al.[160] reported the synthesis of PVDF- $b$-PNVP diblock copolymers via a sequential RAFT/MADIX polymerization, first obtaining a PVDF-xanthate macroinitiator which was further used as the macromolecular CTA for the radical polymerization of NVP (Scheme 29). 

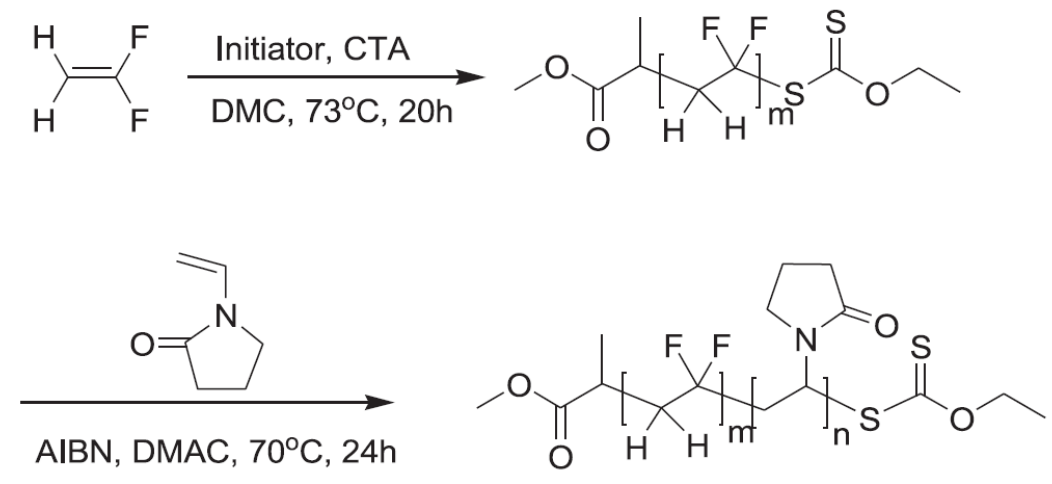

Scheme 29. Synthesis of PVDF- $b$-PNVP block copolymer by RAFT copolymerization of VDF with N-vinylpyrrolidone (NVP).[160] Reproduced with permission from Elsevier.

Another strategy to achieve the VDF-based copolymers uses EVE as the first monomer and VDF as the second monomer. Using this strategy, Guerre et al.[87] prepared unprecedented poly(ethyl vinyl ether)-block-PVDF (PEVE-b-PVDF) block copolymers (Scheme 30) via the sequential combination of cationic RAFT[161] polymerization of vinyl ethers followed by the radical RAFT polymerization of VDF. Besides, their low $T_{g}$ (from the poly(ether) sequence) and rather high melting point $\left(T_{m}\right)$ (from the PVDF block) make them a potential candidate to be used as TPEs. These block copolymers comprising of incompatible polymer segments may have further potential applications due to their phase segregation and self-assembly behavior.
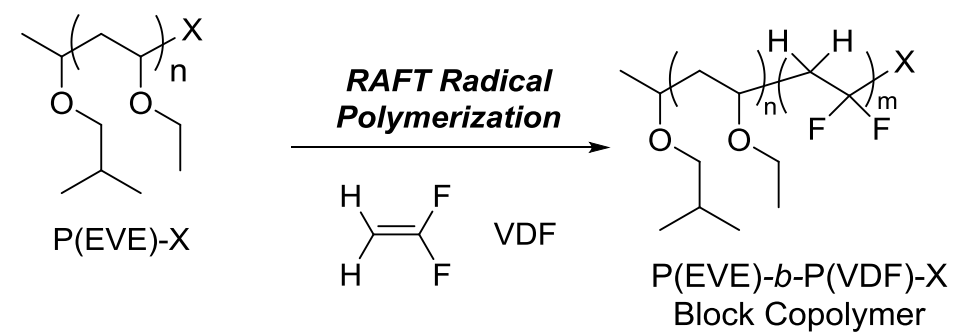

Scheme 30. Poly(Vinyl ether)- $b$-PVDF block copolymers by sequential cationic and radical copolymerizations controlled by xanthates or dithiocarbamates (X moieties).[87] Reproduced with permission from American Chemical Society. 
5.2.4. Direct synthesis and properties of a PVDF- $b$-poly(M) block copolymers Researchers have synthesized various PVDF- $b$-poly(M) copolymers with specific functionalities for various applications. Hydrolysis of the oligo(VAc) segment under acidic $\mathrm{pH}$ produced amphiphilic poly(VDF-co-TFP)- $b$-oligo(vinyl alcohol) diblock copolymers without any dehydrofluorination. The resulting block oligomers act as novel surfactants[83] endowed with low critical micellar concentrations (CMC) and surface tensions $\left(17 \mathrm{mN} \cdot \mathrm{m}^{-1}\right.$ for $0.5 \%$ of copolymer in water), similar to the well-known commercially available fluorosurfactant, perfluorooctanoic acid (PFOA). These block copolymers could emerge as an alternatives to commercially available surfactants such as PFOA and perfluorooctane sulfonic acid (PFOS, regarded as toxic, persistent and bioaccumuble, and even suspected to be mutagenic) due to their small size and the high stability of the perfluorinated groups.[162-164]

A similar strategy was successfully applied for the stepwise copolymerization of VDF with TrFE (and 1234yf) by MADIX copolymerization (Scheme 14) for the development of electroactive polymers.[124] In addition, another comonomer, tert-butyl 2-trifluoromethacrylate (MAF-TBE) (which cannot be polymerized under radical conditions $[150,165]$ was successfully copolymerized with VDF[166], initiated by bis(4-tert-butyl cyclohexyl) peroxydicarbonate and with O-ethyl-S-(1-methyloxycarbonyl) ethyl xanthate as the CTA (Scheme 31), resulting in copolymers with controlled molar masses ranging between 1,500 to 40,000 g.mol ${ }^{-1}$ and $Ð$ lower than 1.8.[122] Subsequent chain extension of these poly(VDF-co-MAF-TBE) copolymers bearing a xanthate end-group was carried out either with VAc or VDF to produce poly(VDF-coMAF-TBE)- $b$-poly(VAc) and poly(VDF-co-MAF-TBE)- $b$-PVDF block copolymers, respectively. This also evidences the presence of xanthate end-group in the poly(VDF-co-MAF- 
TBE) chain.

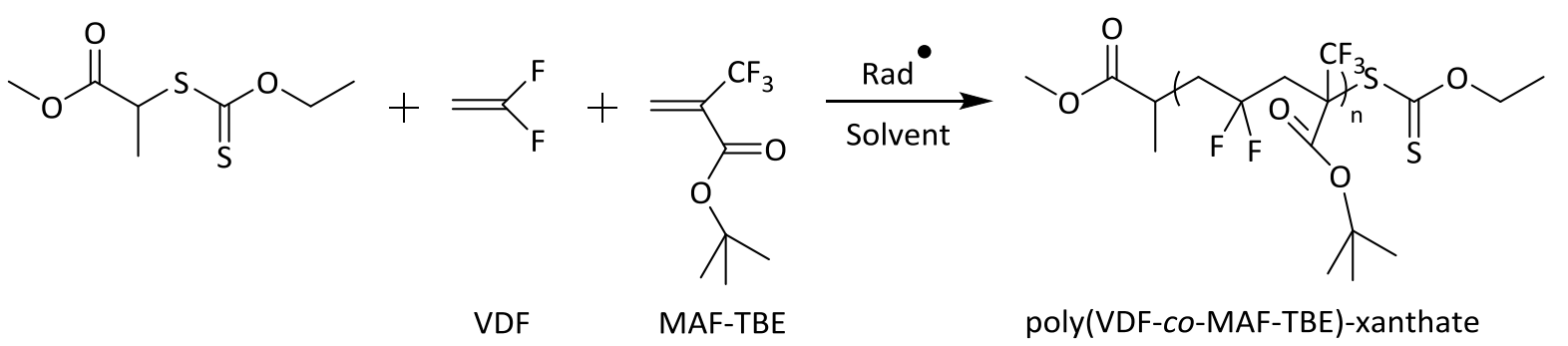

Scheme 31. Radical copolymerization of VDF with MAF-TBE controlled by O-ethyl-S-(1methyloxycarbonyl) ethyl xanthate.[122] Reproduced with permission from Royal Society of Chemistry.

Interestingly, such synthesized VDF-based block copolymers have successfully been employed as compatibilisers for the preparation of commercially available poly(VDF-co-HFP) copolymer (Kynar® marketed by Arkema) and functional triethoxysilane as novel proton exchange membrane fuel cells by reactive extrusion (see Section 9.2.3).[167] The modulated composites involved the in situ generation of the inorganic phase [via sol-gel chemistry from (3mercaptopropyl)triethoxysilane]. For the best performance, the copolymer compatibilizer was incorporated at the interface between the organic and inorganic phases.

\subsubsection{RAFT (co)polymerization of fluoromonomers controlled by trithiocarbonates}

Wang's team[168] synthesized a telechelic poly(VDF-co-HFP) copolymer by RDRP and then used it as a macroCTA for the subsequent RAFT polymerization of an imidazolium methacrylate to prepare a series of triblock copolymers composed of a central poly(VDF-coHFP) sequence and ionic liquid moieties composed of poly(imidazolium methacrylate) segment (Scheme 32). The authors observed that due to the enhanced segmental mobility, the polymers bearing trifluoromethylsulfonimide counterions displayed higher ionic conductivities than their 
$\mathrm{BF}_{4}{ }^{-}$homologues. The temperature dependence of the ion mobilities of the triblock copolymers, as described using a Vogel-Tamman-Fulcher equation, indicates a strong correlation between ion conduction and polymer segmental dynamics.[168]

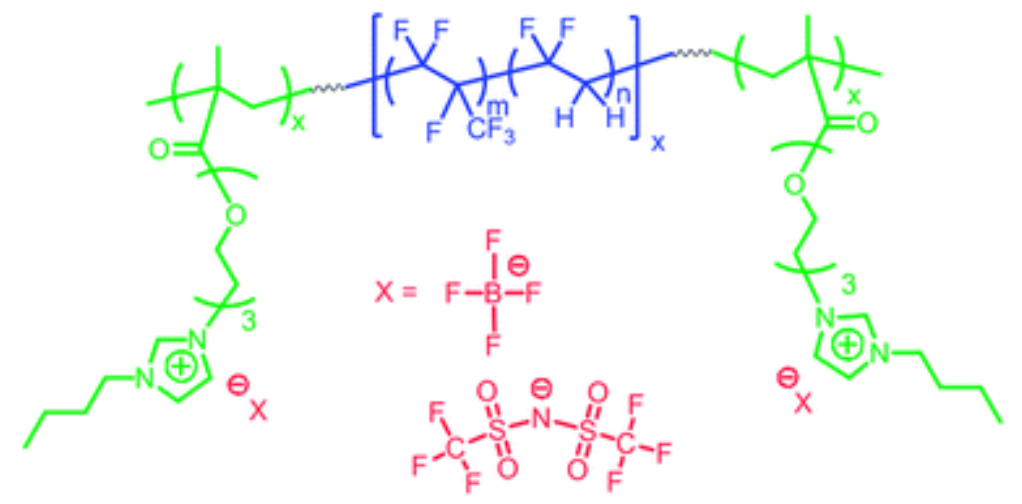

Scheme 32. Schematic representation of the poly(imidazolium methacrylate)- $b$-poly(VDF-coHFP)- $b$-poly(imidazolium methacrylate) block terpolymer with trifluoromethylsulfonimide counterions by sequential RAFT copolymerization.[168] Reproduced with permission from Royal Society of Chemistry.

\subsubsection{Copolymers of CTFE with ethyl vinyl ether}

Guerre et al. reported of unprecedented CTFE-containing block copolymers (PEVE- $b$-P(CTFEalt-EVE)), where EVE stands for ethyl vinyl ether, via sequential cationic and radical RAFT copolymerizations (Scheme 33)[169] following two synthetic pathways. Pathway 1: synthesis of a PEVE block by cationic RAFT polymerization initiated from a poly(CTFE-alt-EVE) macro initiator, which was prepared by radical RAFT copolymerization, Pathway 2: synthesis of a poly(CTFE-alt-EVE) block by radical RAFT copolymerization from a PEVE macroCTA, prepared via controlled cationic RAFT polymerization. Results revealed that both xanthate and dithiocarbamate RAFT agents provide efficient control over the radical RAFT copolymerization of CTFE and EVE. DSC analysis revealed the immiscibility of PEVE and poly(CTFE-alt-EVE) 
sequences in the block copolymers by the presence of two $T_{g} \mathrm{~s}$.

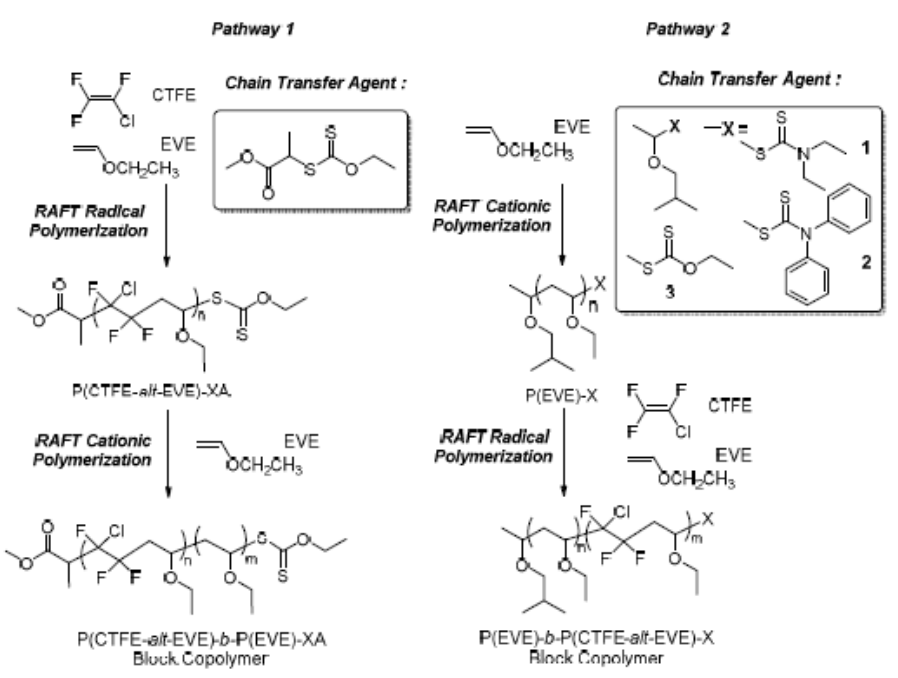

Scheme 33. Synthesis of poly(CTFE-alt-EVE)- $b$-PEVE and PEVE- $b$-poly(CTFE-alt-EVE) block copolymers using pathways 1 and 2.[169] Reproduced with permission from Royal Society of Chemistry.

\subsection{Block Copolymers via OMRP of fluoroalkenes}

\subsubsection{Synthesis of VDF-based block copolymers using cobalt complexes}

Banerjee et al. reported the synthesis of VDF-based block copolymers (Scheme 34) with low dispersities $(\leq 1.31)$ via OMRP using an alkyl-Cobalt complex (R-Co).[97] The synthesized polymers were characterized by NMR (Figure 14) and SEC analysis. DFT calculations supported the efficient reactivation of both head and tail chain end dormant species. The di-and triblock copolymers exhibited a $T_{g}$ characteristic of the PVAc block $\left(35^{\circ} \mathrm{C}\right)$ and a reduced degree of crystallinity relative to neat PVDF. 


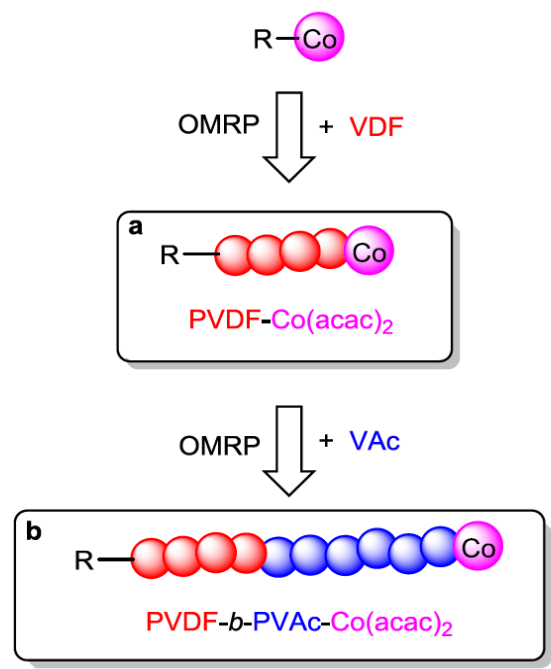

Scheme 34. Synthesis of PVDF and VDF-containing diblock- and symmetrical triblock-like copolymers via $\mathrm{Co}(\mathrm{acac})_{2}$-mediated OMRP at $40{ }^{\circ} \mathrm{C}$.[97] Reproduced with permission from Wiley.

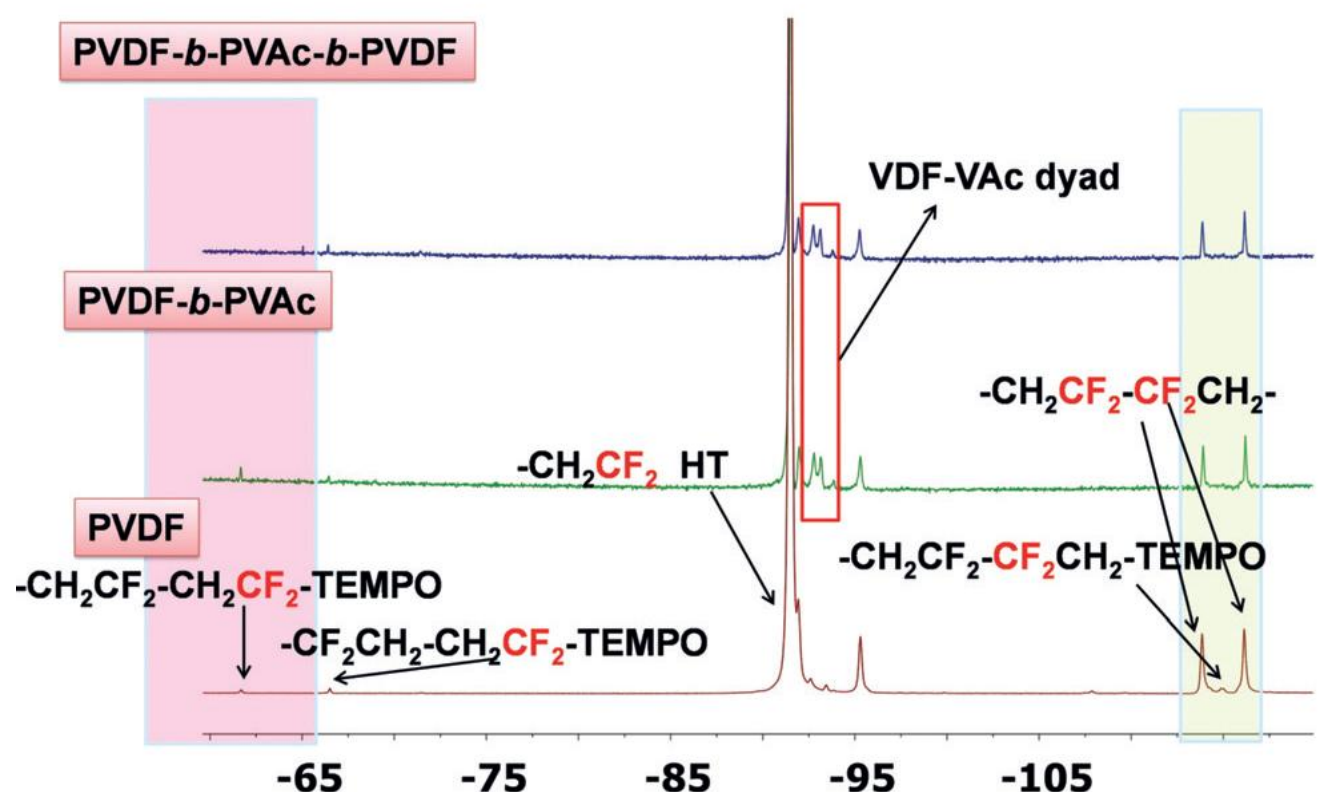

Figure 14. ${ }^{19} \mathrm{~F}$ NMR spectra of PVDF-TEMPO (bottom), of the PVDF- $b$-PVAc-TEMPO diblock copolymer (middle) obtained by cobalt mediated sequential radical copolymerization of VDF and VAc, and of the PVDF-b-PVAc-b-PVDF triblock copolymer (top).[97] Reproduced with permission from Wiley. 
5.3.2. Cobalt-Mediated Radical Copolymerization of chlorotrifluoroethylene and vinyl acetate In a similar strategy, Bai and co-workers[132] reported OMRP of CTFE and VAc mediated by bis(acetylacetonato)cobalt(II) $\left(\mathrm{Co}(\mathrm{acac})_{2}\right)$ and initiated by 2,2'-azo-bis-isobutyronitrile (AIBN). Though the comonomer contents were not supplied and surprisingly CTFE was more reactive than VAc, the molar masses (up to $39700 \mathrm{~g} \mathrm{~mol}^{-1}$ ) and the fluorinated unit contents of the copolymers could be controlled, and a further chain extension polymerization of VAc could lead to a poly(CTFE-co-VAc)- $b$-PVAc block copolymer.

\subsection{Block copolymers via ATRP}

\subsubsection{Synthesis of PVDF containing block copolymers}

Destarac et al.[170] reported the syntheses of PVDF- $b$-poly(M) BCPs where M stands for styrene (St), MMA, methyl acrylate (MA), and n-butyl acrylate (BA) leading to a VDF based copolymer from a dual method comprising the telomerization of VDF with chloroform first, followed by ATRP of such hydrocarbon monomers initiated from the resulting $\mathrm{Cl}_{3} \mathrm{C}-\mathrm{PVDF}$ telomers. The linear $M_{n}$ vs conversion plots of such ATRPs revealed their controlled behavior. This methodology was further used by the same group to obtain PVDF- $b$-fluorinated PS block copolymers.[171] Shi et al. [172] were inspired by such a strategy and prepared block copolymers containing a poly(VDF-co-HFP) block and a poly(styrene sulfonic acid) sequence for potential application as polymer electrolyte membrane for fuel cells. Morphological analysis of the copolymers revealed the formation of phase-separated morphology in the solid state as evidenced by distinct glass transition temperatures.

The same group[173] reported the synthesis of fluorous, block-brush copolymers containing 
VDF, St and ethylene oxide blocks via sequential ATRP technique. Preliminary morphological study by AFM revealed nanophase separated morphology of the block-brush copolymers suggesting the presence of two different phases in their structure. The synthesized polymers might have potential application in functional coatings, as claimed by the authors.

Xu et al.[174] described the ATRP of St from PVDF macroinitiator, synthesized by peroxide initiated radical polymerization of VDF (followed by PS sulfonation). Loos' team suggested various strategies of triblock copolymers.[139] Among them, one example deals with ATRP of tert-butyl methacrylate (tBuMA) with telechelic bis(chloromethyl benzoate) PVDF, followed by hydrolysis into polymethacrylic acid sequences (Scheme 35).[175]

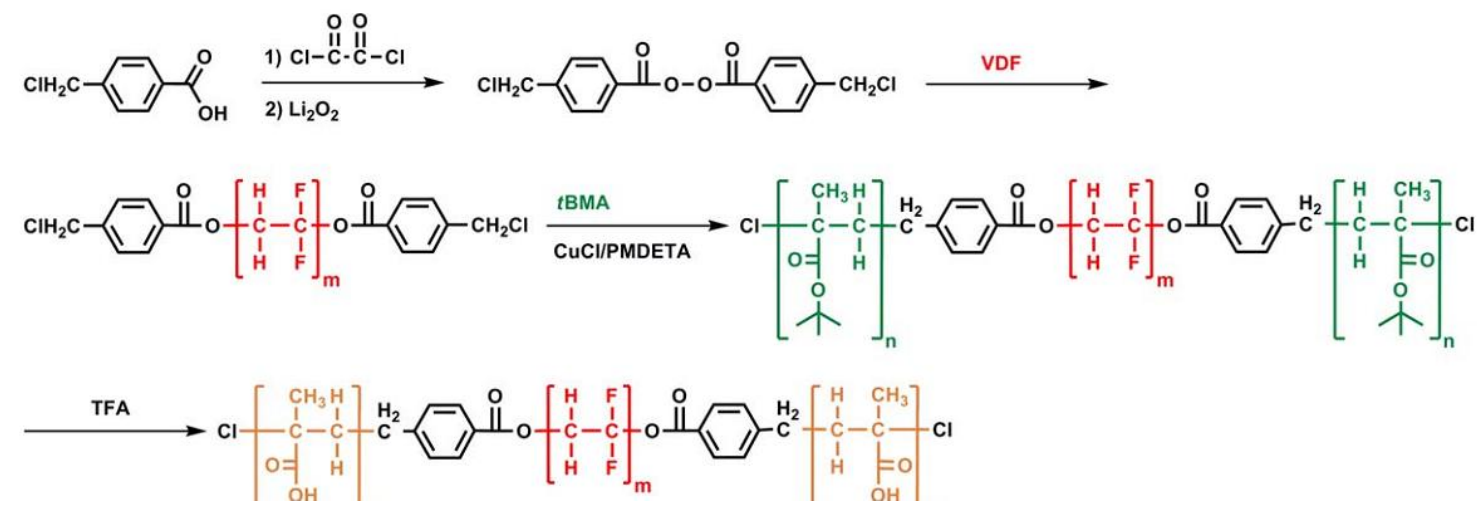

Scheme 35: Synthesis of PtBuMA- $b$-PVDF- $b$-PtBuMA triblock copolymer by ATRP of tertbutyl methacrylate (tBuMA, green) from bifunctional PVDF macroinitiator, produced from the polymerization of VDF initiated by para(chloromethyl) benzoyl peroxide (red). Hydrolysis of PtBuMA into poly(methacrylic acid) (orange) segments yields PMAA- $b$-PVDF- $b$-PMAA.[175] Reproduced with permission from Royal Society of Chemistry.

\subsubsection{Synthesis of fluoroalkene-methyl acrylate copolymers}

Borker and Sen[176] demonstrated the first controlled copolymerization of MA with fluoroalkenes, specially $\mathrm{H}_{2} \mathrm{C}=\mathrm{CH}-\mathrm{C}_{6} \mathrm{~F}_{13}$ (known to be poorly reactive in polymerization), via 
ATRP technique. Results revealed that the rate of the copolymerization and the extent of alkene incorporation into the resulting copolymers were higher than those observed with simple nonfluorinated 1-alkenes. Both the XPS and contact angle measurements of the copolymer films suggested that the fluorinated side chains of the copolymers phase segregated and orient themselves at the air side. This resulted in low surface energy hydrophobic surfaces (water contact angle value, $\left.\mathrm{WCA}=107-118^{\circ}\right)$.

\subsection{Block copolymers via polycondensation}

Dolbier et al.[177] utilized copolycondensation reaction between bis-phenol A and p-bis(chlorodifluoro-methyl)benzene to prepare a novel fluoropolymer (Scheme 36). The authors proposed that this polymerization reaction took place via an unprecedented Unimolecular Radical Nucleophilic Substitution ( $\left.\mathrm{S}_{\mathrm{RN}} 1\right)$ mechanism. The obtained fluoropolymers demonstrated has excellent thermal stability (up to $335^{\circ} \mathrm{C}$ ) and solubility properties.<smiles>FC(F)(Cl)c1ccc(C(F)(Cl)Cl)cc1</smiles>

1

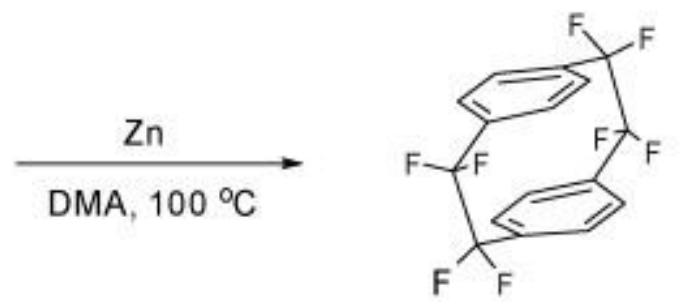

AF4

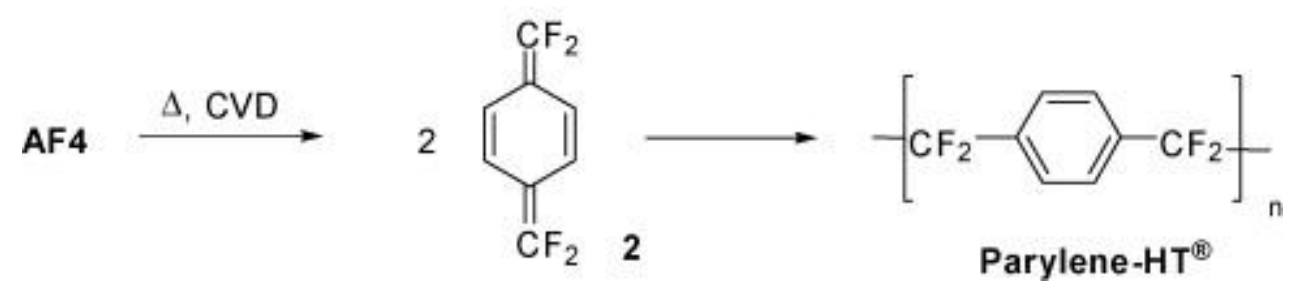

Scheme 36. Synthesis of the copolymer via condensation mechanism, [177] where CVD stands for chemical vapor deposition. Reproduced with permission from Elsevier.

5.6. Block Copolymers via copper alkyne azide cycloaddition (CuAAC) "Click Chemistry" 
Click chemistry[178] is by far is the most facile and selective chemical transformation which simplifies compound synthesis, providing the faster lead discovery and optimization. The click reaction provides wide scope, gives very high yields, generates only inoffensive byproducts that can be easily removed by nonchromatographic methods.[178] Beuermann and coworkers[179] first prepared PVDF-I macroinitiator via ITP of VDF and then converted it into azido functionalized PVDF (PVDF-N ${ }_{3}$ ). Subsequently, the surface of single-walled carbon nanotubes (SWCNTs) was decorated with PVDF-N $\mathrm{N}_{3}$. The resulting material was characterized by dispersibility, Raman spectroscopy, and thermogravimetric analyses to confirm successful functionalization. Disordering of the SWCNTs, as revealed by the Raman spectra suggested covalent attachment of PVDF to SWCNTs. Comparative electrical conductivity analysis revealed that the PVDF films containing 0.5 and 1 wt $\%$ PVDF-functionalized SWCNTs exhibited significantly improved electrical conductivity compared to PVDF films containing pristine SWCNTs.[179]

The same authors subsequently prepared PVDF functionalized carbon black (CB) nanoparticles either by trapping of macroradicals or by cycloaddition (Scheme 37). The effect of temperature, time, polymer concentration, and polymer molar mass on the functionalization reaction of $\mathrm{CB}$ was extensively studied.[180]

$$
\mathrm{nF}_{2} \mathrm{C}=\mathrm{CH}_{2}+\mathrm{IC}_{6} \mathrm{~F}_{12} \mathrm{I} \stackrel{\mathrm{scCO}_{2}}{120{ }^{\circ} \mathrm{C}} \mathrm{I}-\mathrm{PVDF}-\mathrm{I}
$$


Scheme 37. Synthesis of functionalized CB particles.[180] Reproduced with permission from Wiley.

Beuermann's group also reported the synthesis of block copolymers consisting of PVDF and PS using CuAAC. Firstly, propargyl-terminated terminated PS was synthesized via NMP using a propargyl-functionalized alkoxyamine initiator ( $\mathrm{PgOTIPNO}$ ). Then, alkyne-terminated PS with different molar masses was covalently attached onto above synthesized PVDF- $\mathrm{N}_{3}$ via CuAAc to prepare PVDF-b-PS copolymers (Scheme 38).[181] The major issue with this technique was the long reaction time (7 days).

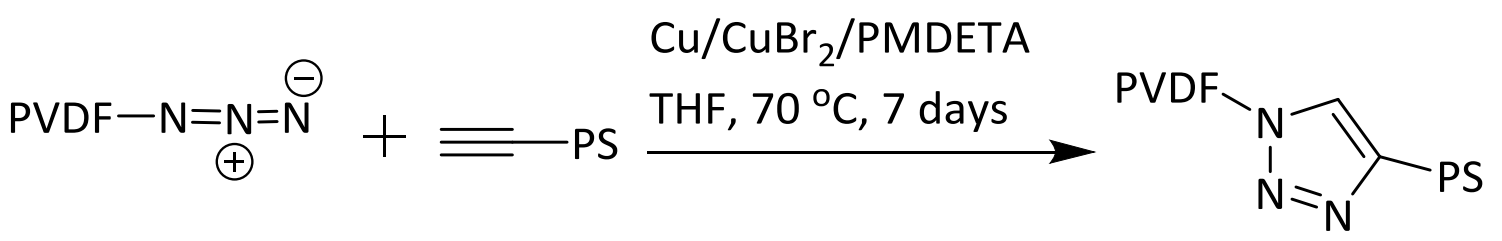

Scheme 38. Synthesis of PVDF- $b$-PS copolymer by CuAAC.[181] Reproduced with permission from Royal Society of Chemistry.

Another strategy was implemented by Guerre et al.[182] who produced various relatively welldefined $\mathrm{BCPs}$ of various chain-lengths $\left(\mathrm{PVDF}_{40}-b-\mathrm{PDMAEMA}_{23}, \mathrm{PVDF}_{40}-b-\mathrm{PDMAEMA}_{69}\right.$, PVDF $_{40}-b$-PDMAEMA 162 ), with low dispersities $(\nexists<1.55)$ (Scheme 39). PVDF- $b$-PDMAEMA BCPs, synthesized by CuAAC click coupling of azide-functionalized PVDF obtained by RAFT polymerization of VDF and alkyne-functionalized PDMAEMA produced by ATRP and the authors also studied the structure-morphology relationship. In particular, such amphiphilic BCPs displayed a self-assembled behavior in water at $\mathrm{pH} \mathrm{2,} 8$ (native $\mathrm{pH}$ ) and 10 and were polydisperse (20-500 nm, Figure 15), as spherical aggregates. However, at $\mathrm{pH} 8, \mathrm{PVDF}_{40}-b$ - 
PDMAEMA $_{69}$ also formed micrometer-long rigid cylindrical micelles. These morphologies were relevant examples of BCP nanostructures based on PVDF achieved by crystallization-driven self-assembly.

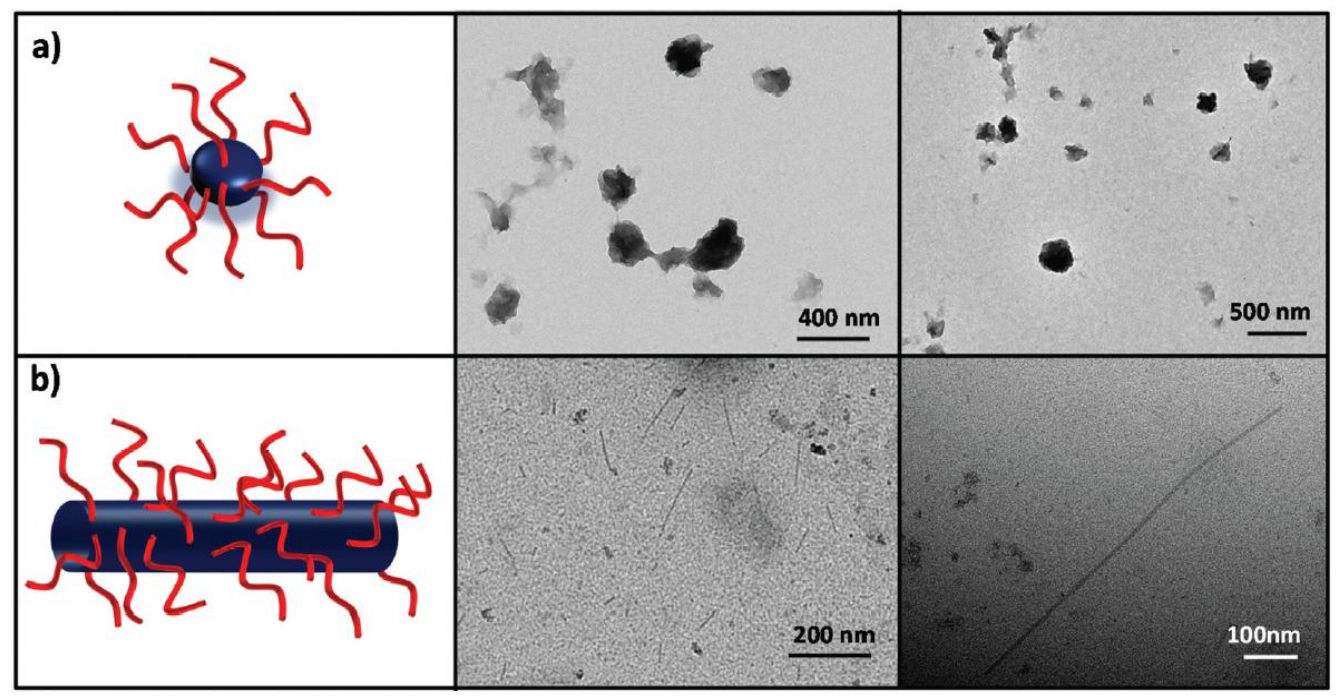

Figure 15. Representative TEM images of morphologies formed by self-assembly in water at $\mathrm{pH}$ $=8$ from: (a) $\mathrm{PVDF}_{40}-b-\mathrm{PDMAEMA}_{23}$, (b) $\mathrm{PVDF}_{40}-b$-PDMAEMA 69 BCPs.[182] Reproduced with permission from Royal Society of Chemistry. 


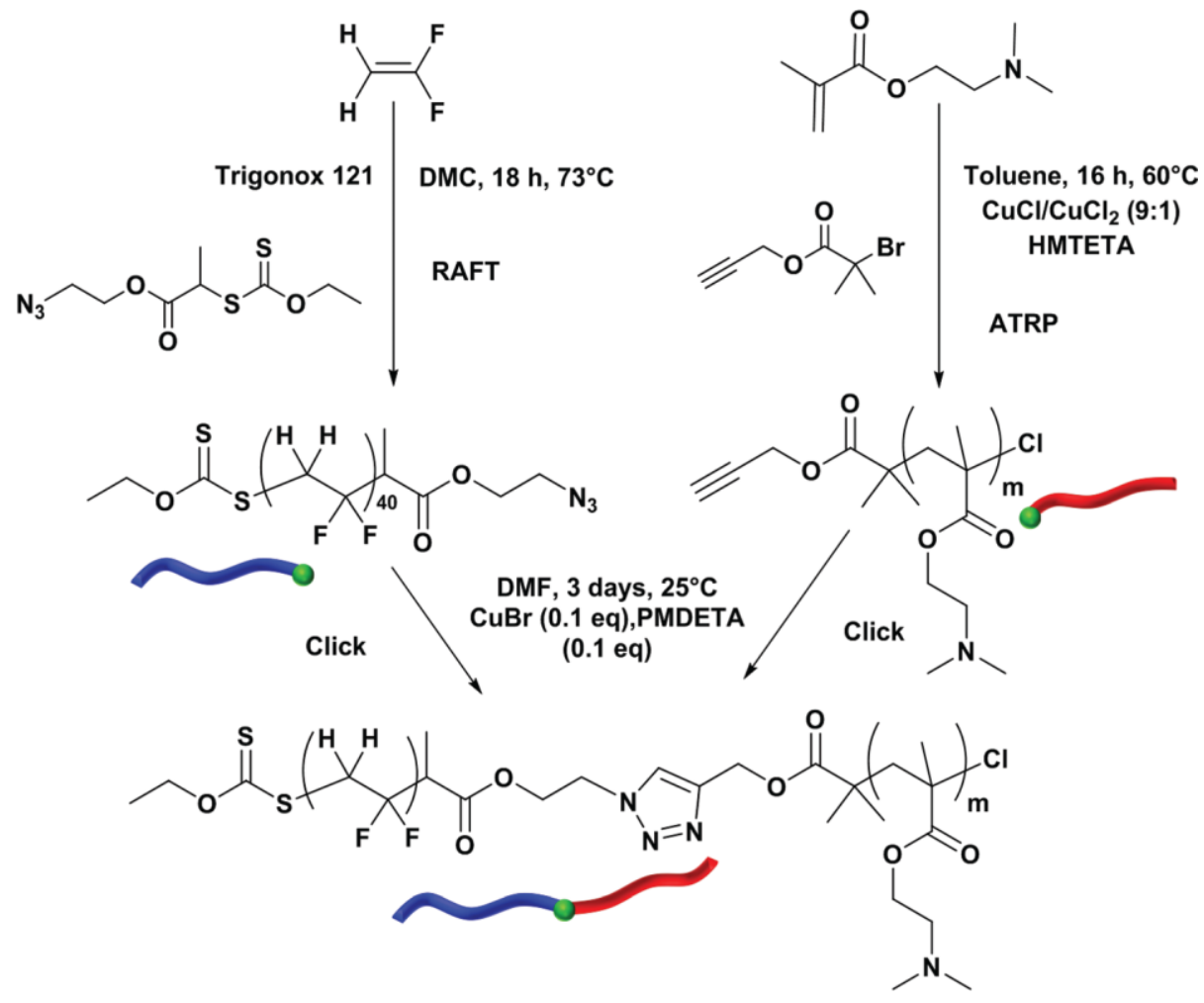

Scheme 39. Synthesis of PVDF- $b$-PDMAEMA Block Copolymers by CuAAc clickcoupling.[182] Reproduced with permission from Royal Society of Chemistry.

Loos' team[183] recently synthesized and comprehensively studied the structure-electroactive properties of original PS- $b$-poly(VDF-co-TrFE)- $b$-PS and P2VP- $b$-poly(VDF- $c o-T r F E)-b$-P2VP triblock copolymers (where 2VP stands for 2-vinylpyridine). These terpolymers were produced by CuAAc click-coupling of telechelic bis(azide) terminated poly(VDF-co-TrFE) copolymers with alkyne terminated P2VP or PS obtained by RAFT polymerization of VP or styrene in the presence of an $\omega$-alkyne trithiocarbonate (Scheme 40). Surprisingly, low $Ð$ values (1.51) were obtained for a conventional radical copolymerization of VDF and TrFE. Though (i) keeping ester function from benzoyl peroxide, (ii) the absence of evidence of the control of the RAFT of Sty and $2 \mathrm{VP}$, and (iii) the functionality of 2.00 are questionable, the goal of such a study was to obtain original electroactive materials with tunable responses. Thus, the different behaviors, ferroelectric, antiferroelectric-like and linear dielectric, readily achieved by simple adjustment of 
the polarity of both blocks, showed the vast potential of such block copolymers for numerous applications in field of piezo- and ferroelectrical devices. Both the presences of PS and P2VP onto the electric properties were compared and discussed.

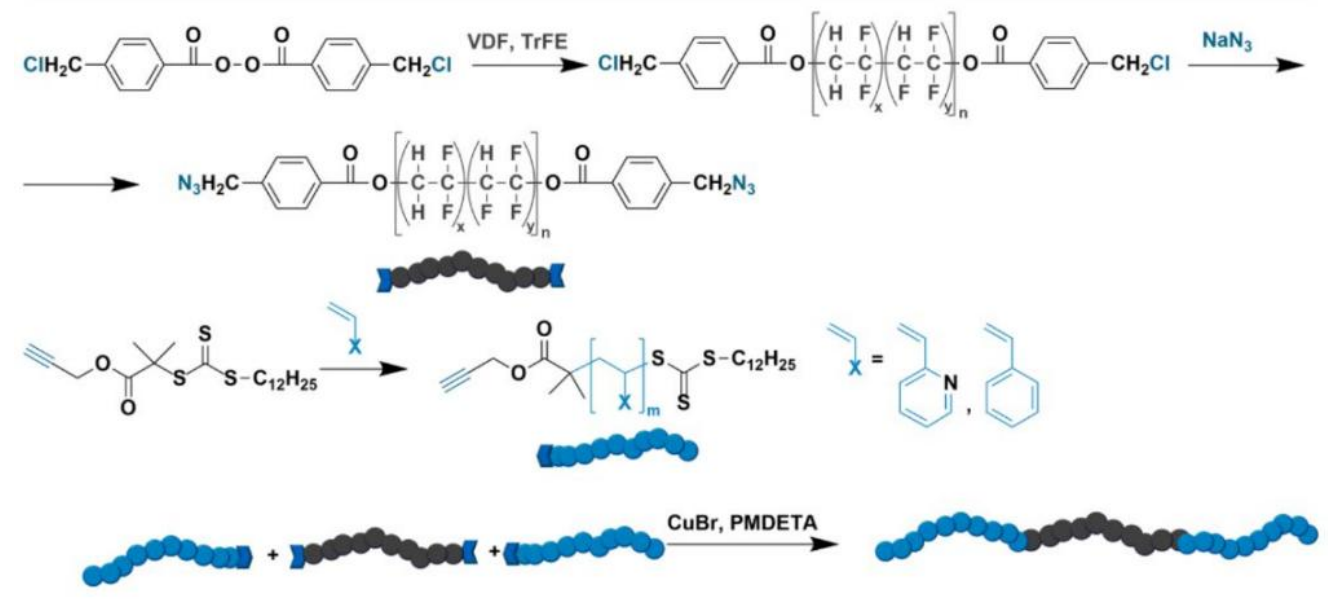

Scheme 40: Synthetic strategy for the preparation of poly(M)-b-poly(VDF-co-TrFE)-b- poly(M) triblock copolymers (where M stands for styrene or vinylpyridine) by CuAAc click-coupling of telechelic azide terminated poly(VDF-co-TrFE) and alkyne terminated P2VP or PS obtained by RAFT polymerization of VP or styrene.[183] Reproduced with permission from Nature Publishing House.

In addition, these authors[184] recently used a "reversed" strategy involving the RAFT polymerization of $2 \mathrm{VP}$ in the presence of an azido CTA clicked with a telechelic bis(alkyne) poly(VDF-co-TrFE) copolymer (Scheme 41) and studied the effect of the morphology characteristics of the block copolymers onto ferroelectric responses, reported for the first time. 


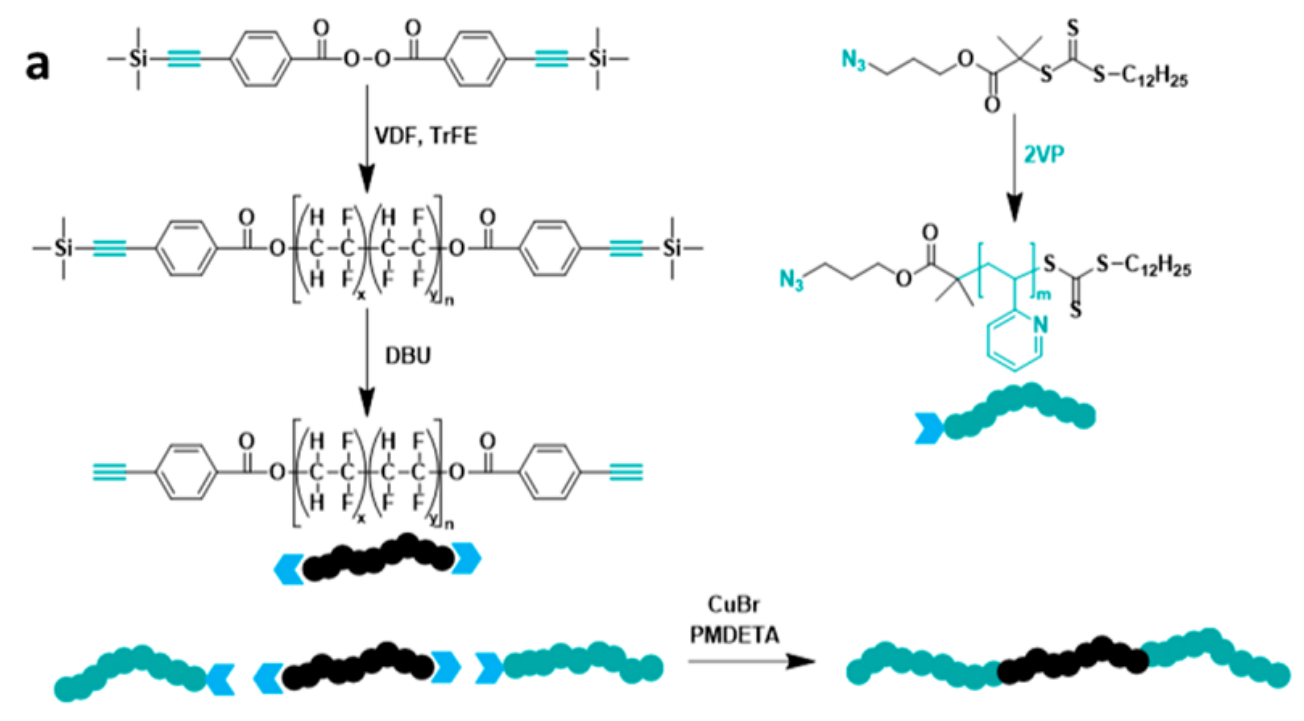

Scheme 41. Schematic representation of the synthesis of poly(VDF-co-TrFE)-based block copolymers using CuAAc click coupling of telechelic alkyne-terminated poly(VDF-co-TrFE) and azide-terminated P2VP.[184] Reproduced with permission from American Chemical Society.

By simple adjustment of the ratio between the blocks, the structures in the melt varied from lamellae, cylindrical and spherical morphologies (Figure 16), well supported by SAXS and WAXS experiments, and persevered upon crystallization. The authors featured that the high poly(VDF-co-TrFE) central block content crystallization became dominant and drove the selfassembly of the block copolymers. The authors studied the effect of morphological characteristics of such block copolymers on the ferroelectric response and based on the selfassembly of P2VP- $b$-poly(VDF-co-TrFE)- $b$-P2VP triblock copolymers, ferroelectric nanostructures were produced. 


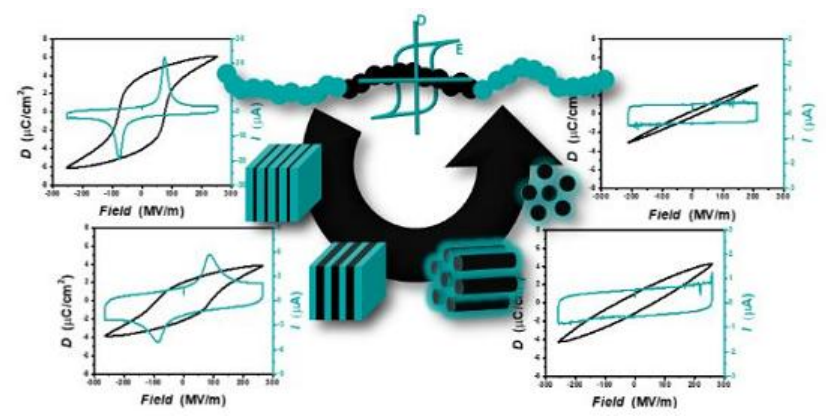

Figure 16. Lamellae, cylindrical and spherical morphologies, and displacement-electric field loops for different structures based on various chain lengths in P2VP- $b$-poly(VDF-co-TrFE)- $b$ P2VP triblock copolymers.[184]) Reproduced with permission from American Chemical Society.

Terzic et al.[185] recently synthesized homopolymers of PVDF functionalized by alkyne or azide end groups using functional benzoyl peroxide initiators. These polymers were used for the preparation of different triblock copolymers based on polystyrene (PS), poly(tert-butyl acrylate), $\mathrm{P}^{t} \mathrm{BA}$, and poly $\mathrm{N}$-isopropyl acrylamide (PNIPAAm) sequences using CuAAC. This synthetic route proposed an alternative method to RAFT and ITP for the formation of well-defined block copolymers, though transfer reactions to initiator, monomer or solvent should occur. Using the same methodology, this Dutch group also reported other ABA triblock copolymers (where B represents PVDF and A stands for polystyrene, polyNIPAAM or PtBu), their morphologies and tunable crystallizations.[185] These triblock copolymers were achieved from the Huisgen CuAAC reaction between a telechelic bis(alkyne) PVDF and azido terminated poly(styrene), PNIPAAM and poly(tert-butyl acrylate) iome(PtBA) produced from RAFT.

\subsection{Conclusion}


Different RDRP techniques have been developed over the last decades for the synthesis of fluorinated block copolymers containing fluorinated alkenes. Historically, ITP has been the more versatile route, opening the way to industrial production of Hard-Soft-Hard thermoplastic elastomers that keep their good performance over a wide range of temperatures. More recent strategies as RAFT/MADIX and CMRP also show promising results, supported by theoretical calculations. Though most studies involve VDF as monomers, some more recent works have used TrFE, and CTFE and HFP with vinyl ethers. Thus synthesized block copolymers have controlled macromolecular architectures and, amphiphilic BCPs led to surfactants and enabled self-assemblies into various nanoobjects (Section 8.7).

\section{Synthesis of fluorinated graft copolymers}

As for block copolymers, graft copolymers are well-defined materials quite useful for many applications (hot melts, adhesives, emulsifiers for plastics, impact resistance additives, etc). A graft copolymer is a branched polymer that contains one or more side chains being different from the backbone. Such heterogeneous sequences show properties of both (or more) the polymeric backbone and the pendant chains rather than averaging the homopolymer properties. A twophase graft copolymer is expected to display a suitable miscibility of each homopolymers and have the desirable properties of being located at the interface of both phases. Many authors have studied various strategies to prepare fluorinated graft copolymers, where the backbone is fluorinated and the graft halogenated or not. Several routes are possible (Scheme 42). 


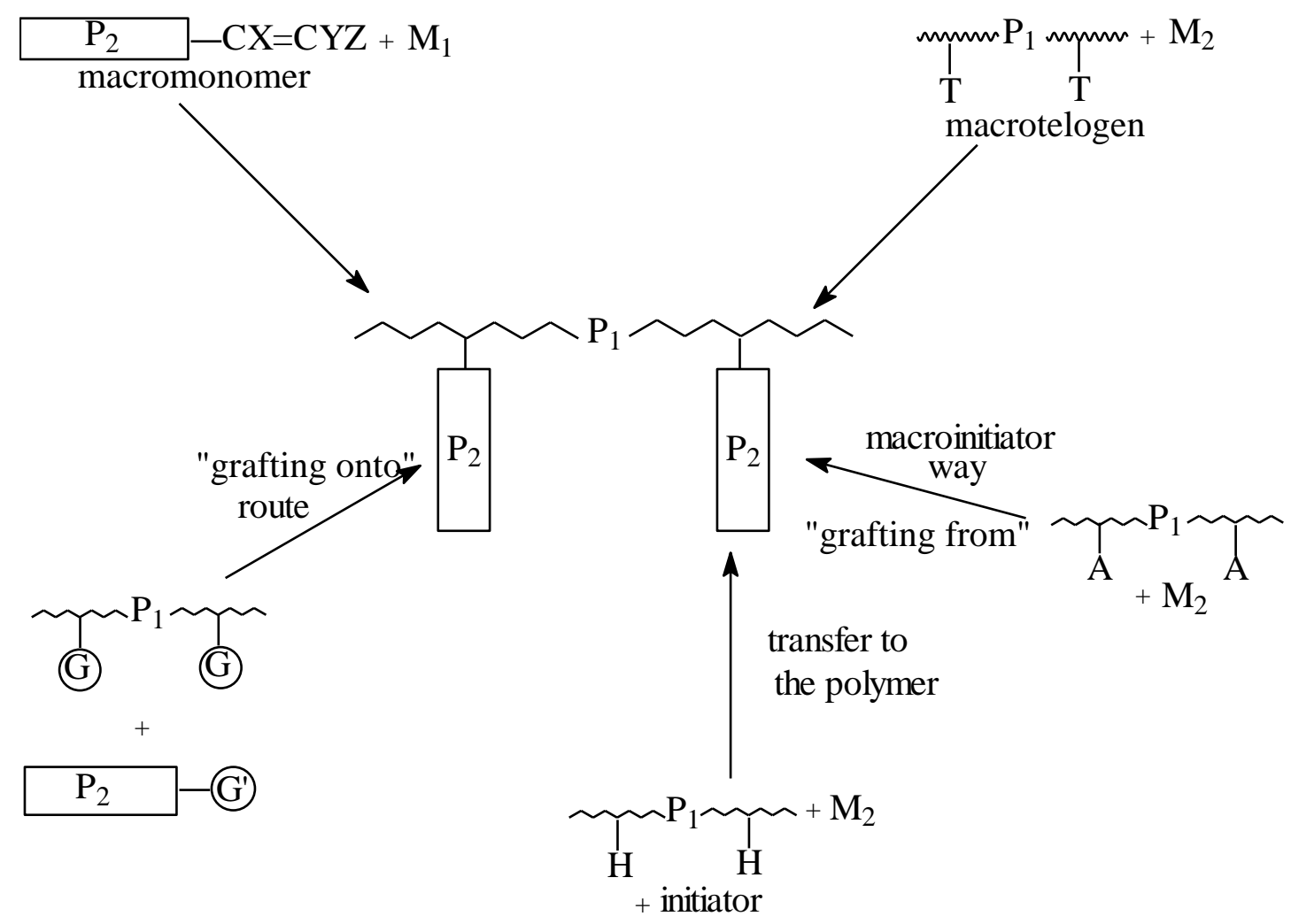

$\mathrm{T}:$ transfering group

A : initiator group

Scheme 42. Various strategies to prepare fluorinated graft copolymers.

For fluoropolymers, the most relevant technique is the activation of a commercially available fluoropolymer followed by a grafting from its backbone through more recent strategies involving the chemical modification of such a fluoropolymer.

6.1. Fluorinated graft copolymers by radiografting

The radio grafting technique (from ozone, gamma or $\mathrm{X}$ rays, electron beam or ${ }^{60} \mathrm{Co}$ ) of a fluoropolymer (Scheme 43) usually leads to generated peroxides or hydroperoxides able to initiate the graft polymerization of a $\mathrm{M}$ monomer. These have been extensively studied for membrane applications, as polymer electrolyte membranes for fuel cells, gel polymer 
electrolytes for Li-ion batteries, functional (e.g. antifouling or antibacterial) coatings and was comprehensively summarized last decade in a book.[5]

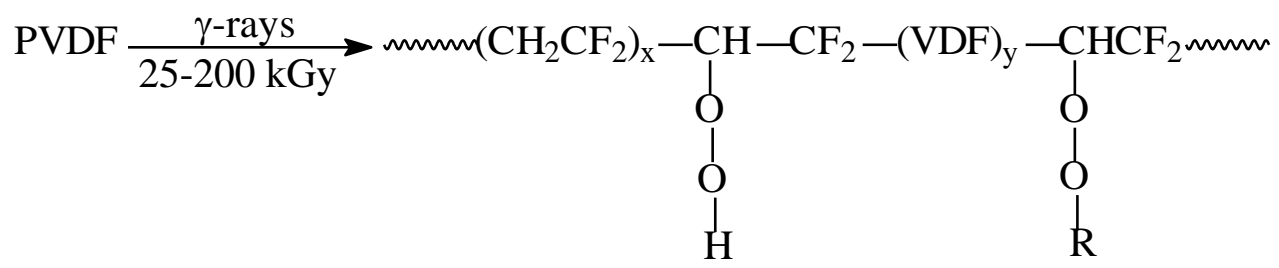

Act-PVDF

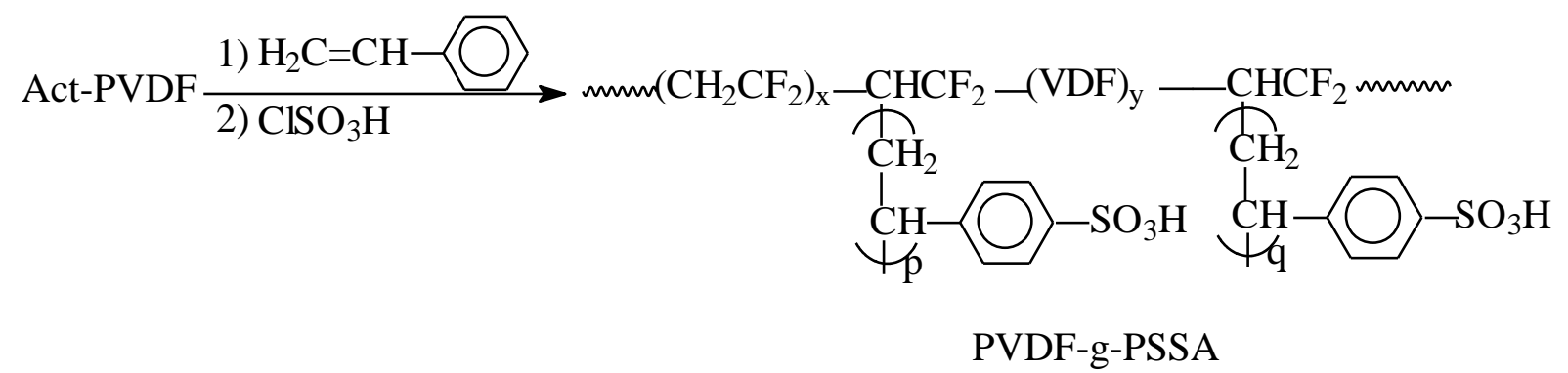

Scheme 43. representative PVDF- $g$-PSSA graft copolymer from radiografting PVDF under gamma rays followed by graft polymerization of styrene which was then converted to sulfonic acid, SSA, from the in situ generated peroxides or hydroperoxides.

Generally, PVDF-graft-poly(styrene sulfonated acid), PVDF-g-PSSA, exhibits a lower methanol permeability than commercially available Nafion ${ }^{\circledR}$. This problem was overcome by Huang et al.[186] who identified the phase separation of PVDF and PSSA graft using of a tapping mode atomic force microscope (AFM) and a high angle annular dark-field scanning transmission electron microscope (Figure 17). Elucidation of the microstructural changes in the membrane with and without methanol immersion revealed that PVDF-g-PSSA copolymer has ionic aggregates with a more stable microstructure than the commercially available Nafion®. 

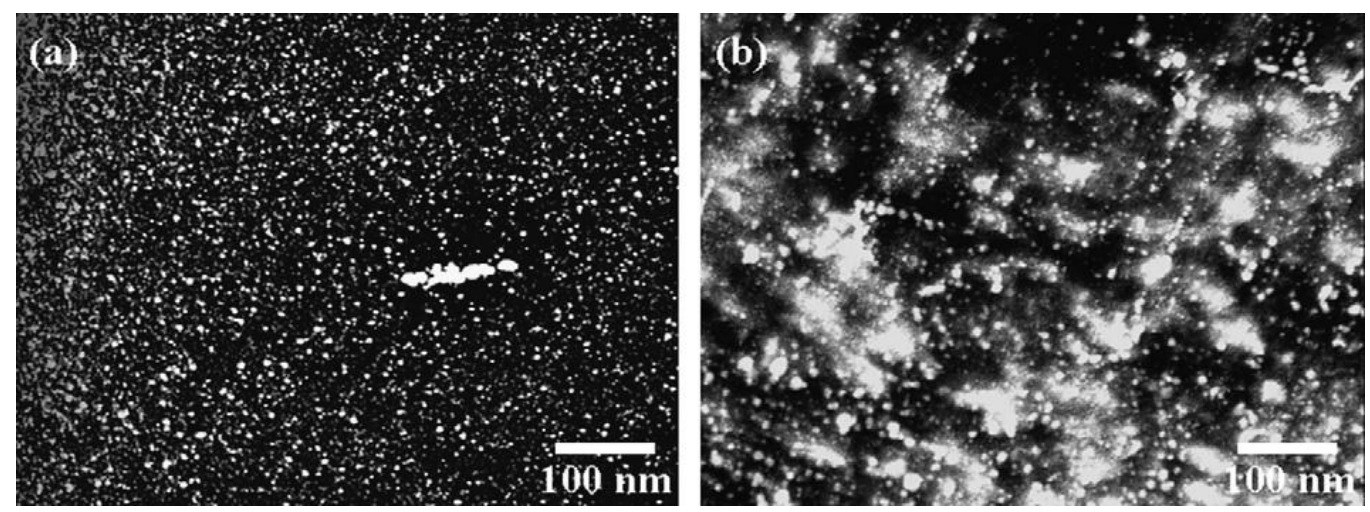

Figure 17. The High-angle annular dark-field imaging (HAADF, an STEM technique) images of (a) $\mathrm{Ag}^{+}$-stained Nafion 117 and (b) $\mathrm{Ag}^{+}$-stained PVDF-g-PSSA.[186] Reproduced with permission from Elsevier.

More recently, Danks et al.[187] synthesized PVDF-g-PVBC graft copolymers via activation of PVDF by $\gamma$-rays $\left({ }^{60} \mathrm{Co}\right)$ followed by the grafting of $p$-chloromethylstyrene (Scheme 44$)$. The degree of grafting was either $38 \%$ or $54 \%$, depending on the grade of PVDF used, while the average thickness was $54 \mu \mathrm{m}$. Such graft copolymers were used for solid alkaline fuel cells after modification of the chloromethyl functionalities into ammonium groups.

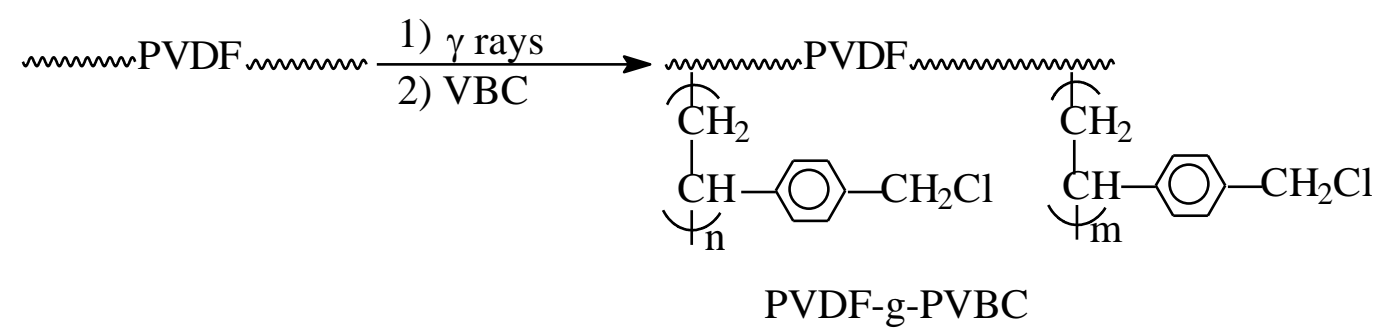

Scheme 44. Synthesis of PVDF- $g$-PVBC graft copolymers via activation of PVDF by $\gamma$-rays $\left({ }^{60} \mathrm{Co}\right)$ followed by the grafting of $p$-chloromethylstyrene.[187] Reproduced with permission from Royal Society of Chemistry. 
An experiment to elucidate the microstructural changes in the membrane with and without methanol immersion shows that PVDF-g-PSSA has ionic aggregates with a more stable microstructure than Nafion®. Liu et al.[188] reported PVDF-based porous membranes via grafting of poly(ethylene glycol) methyl ether methacrylate (PEGMA) onto the surface of PVDF porous membrane. The degree of grafting was significantly influenced by the $\mathrm{pH}$ value of the reaction solution. These PEGMA grafts enabled to increase the hydrophilicity and anti-fouling properties. Cai et al.[189] synthesized PVDF- $g$-PPMA copolymers with pendant alkyne moieties in the PPMA side chains via graft copolymerization of propargyl methacrylate (PMA) from the ozone-preactivated PVDF backbones. The microporous membranes prepared from the PVDF- $g$ PPMA copolymers of different graft concentrations by phase inversion in an aqueous medium showed enrichment of the propargyl groups on the membrane and pore surfaces. Subsequently, the electrolyte-responsive PVDF-g-P[PMA-click-MPS] membranes were prepared via thiol-yne click reaction with 3-mercapto-1-propanesulfonic acid sodium salt on the microporous PVDF- $g$ PPMA polymer membranes. Results revealed that the permeability of aqueous solutions through the synthesized membranes was dependent on the electrolyte concentration.

Kader et al.[190] synthesized gel polymer electrolytes (GPEs) using a UV irradiation method for improving the performances of lithium ion batteries using PVDF-g-poly(tert-butyl acrylate) (PVDF- $g$ - $t \mathrm{BA}$ ) microporous mats. Result revealed that the ${ }^{\mathrm{t}} \mathrm{BA}-$ grafted PVDF microporous electrolytes are potential and promising candidates as gel polymer electrolytes for lithium- ion polymer batteries. Results revealed that the crystallinity of PVDF influences the ionic conductivity, interfacial stability and cyclic performance of the Li/S cells.

Albert et al.[191] prepared radiation grafted membranes by co-grafting styrene, acrylonitrile, and 1,3-diisopropenylbenzene monomers into preirradiated $50 \mu \mathrm{m}$ poly(ethylene-co- 
tetrafluoroethylene) base film, followed by sulfonation in order to introduce proton exchange sites (Scheme 45). The authors also evaluated the membranes for electrolyzer applications. The three key parameters they evaluated are gas crossover, area resistance, and mechanical properties.

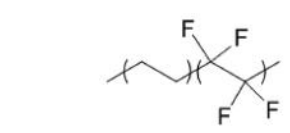

Ethylene tetrafluoroethylene (ETFE)

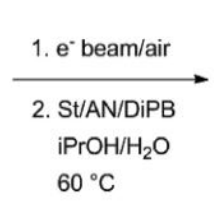

$60{ }^{\circ} \mathrm{C}$
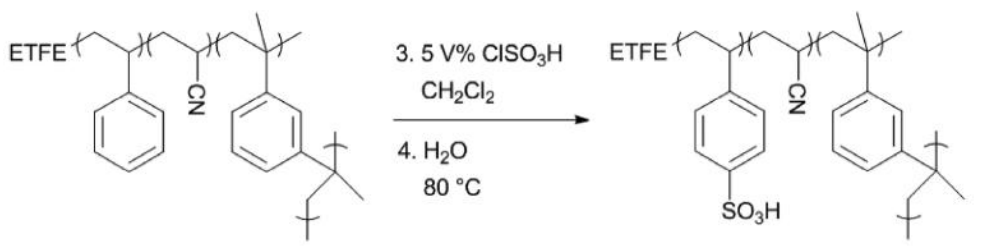

Radiation grafted membrane

Scheme 45. Reaction scheme for synthesizing radiation-grafted membranes where St, AN and DiPB stand for styrene, acrylonitrile and 1,3-diisopropenylbenzene, respectively.[191] Reproduced with permission from American Chemical Society.

\subsection{Graft copolymers from TEMPO or nitroxide groups via NMP}

Sundholm and coworkers developed proton-conducting membranes by direct sulfonation and investigated the effect of radicals and radical decay on the sulfonation of poly(vinyl fluoride) (PVF) films.[192] Results revealed that the most significant parameters are the type of irradiation and radical decay. In contrast, the combination of sulfonation time and chlorosulfonic acid concentration have little or no influence on the final outcome. Therefore, when preparing for example homogeneously sulfonated membranes, the sulfonation reagent concentration must be rather low to avoid over-sulfonation of the surface (Figure 18). 


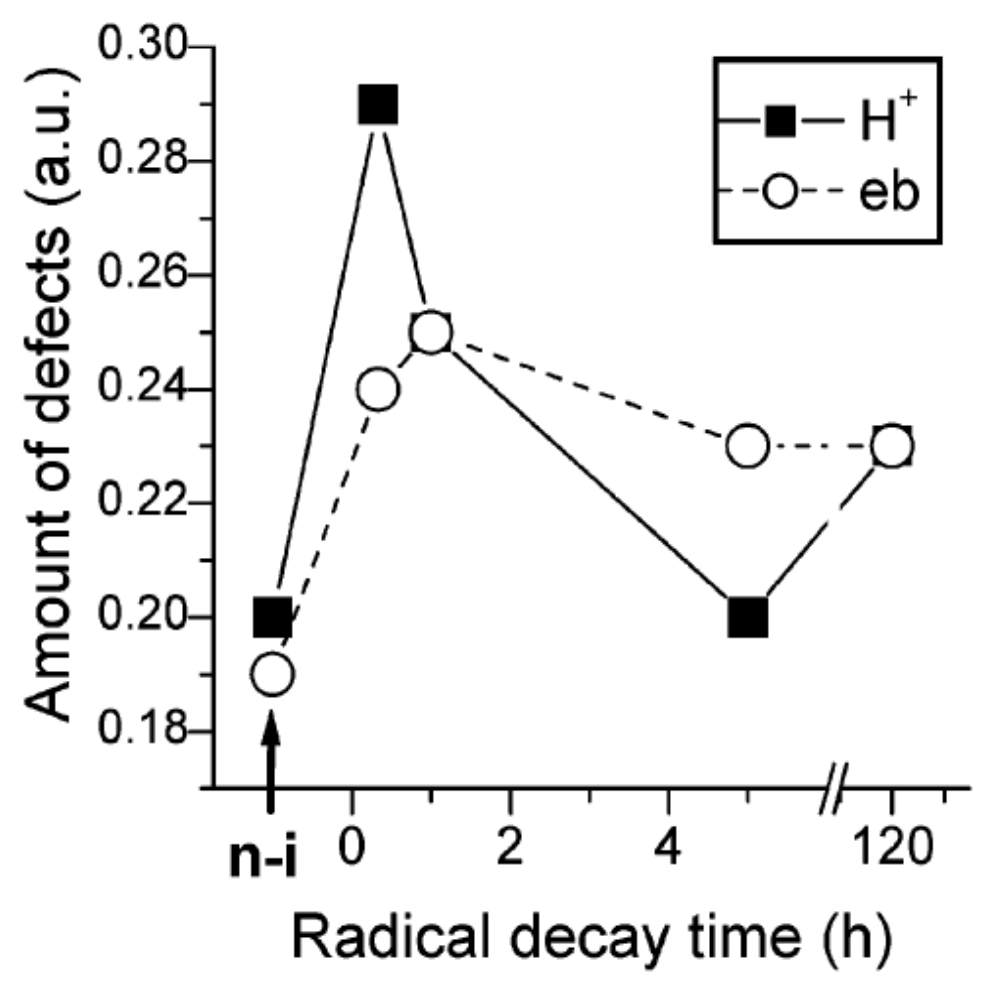

Figure 18. Amount of structural defects as a function of irradiation type and radical decay time. Absorbed dose of both samples was $400 \mathrm{kGy}$. eb, electron beam; H+, proton beam.[192] Reproduced with permission from American Chemical Society.

Another way was suggested by Lienafa et al.[193] who grafted nitroxides onto PVDF under mild conditions. First, the authors achieved the ITP of VDF in the presence of 1-iodoperfluorohexane that led to low molar mass PVDFs followed by the grafting of nitroxide moieties onto PVDF backbone in solution without any polymer activation (Scheme 46). The obtained PVDFs containing dangling nitroxide functions were characterized by FT-IR spectroscopy (Figure 19) and SEC, and acted as efficient macroinitiators for the polymerization of styrene to produce PVDF-g-PS graft copolymers under NMP conditions. 


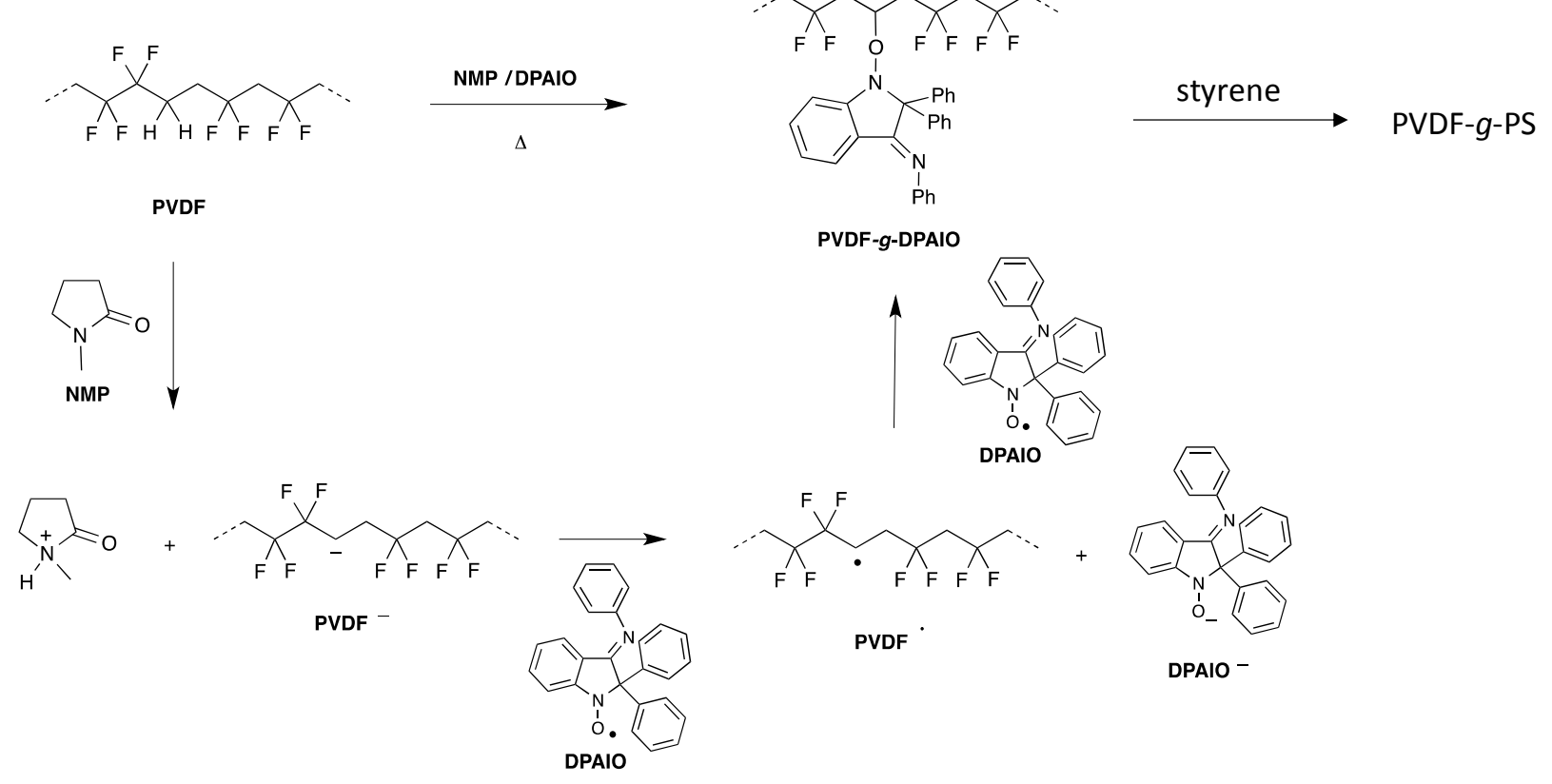

Scheme 46. Sketch of the proposed mechanism for the grafting of 2,2-diphenyl-3-phenylimino2,3-dihydroindol-1-yloxyl nitroxide (DPAIO) onto PVDF in presence of NMP.[193] Reproduced with permission from Elsevier.

The IR spectrum (Figure 19) shows new absorption bands attributed to the aromatic rings characteristic of DPAIO.
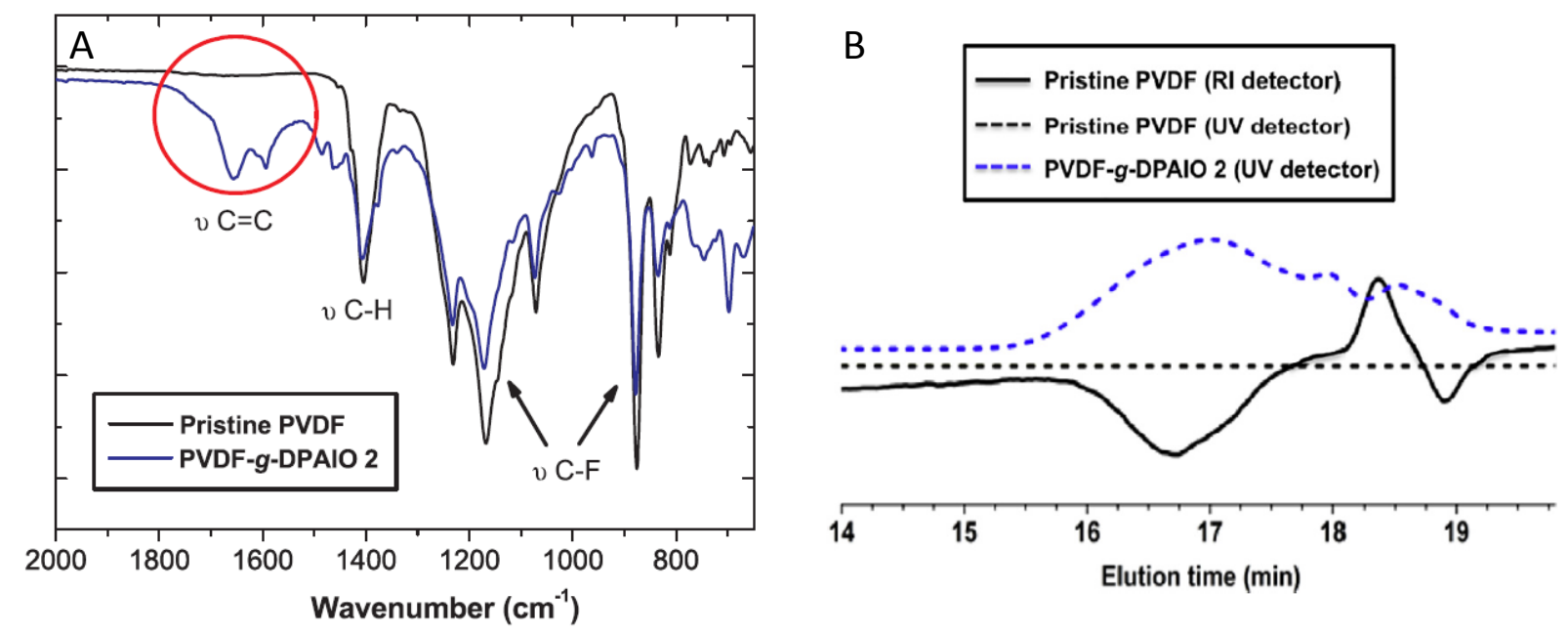
Figure 19. FTIR-ATR spectra (A) and SEC traces (B) of $\mathrm{PVDF}_{21}$ and PVDF-g-DPAIO 2.[193] Reproduced with permission from Elsevier.

Chen et al.[194] developed thermally self-curable poly(VDF-co-CTFE) fluoroelastomer functionalized with pendant 2,2,6,6-tetramethylpiperidinyl-1-oxy (TEMPO). This strategy involves two competitive processes, including the coupling reaction between poly(VDF-coCTFE) macroradicals and TEMPO, followed by the dehydrochlorination of commercially available poly(VDF-co-CTFE).

The above paragraphs confirm the fact that NMP can be a suitable technique for the synthesis of fluorinated graft copolymers for various applications including functional coatings or as emulsifiers for polymer blends.

\subsection{Graft copolymers via RAFT/MADIX polymerization}

Peng et al.[195] prepared PVDF-graft-poly(n-vinyl-2-pyrrolidone) copolymers prepared via a RAFT polymerization from the ozone preactivated PVDF. Quaternization of the tertiary amine groups of the PDMAEMA brushes in the graft copolymers (Scheme 47) enabled to bring both antifouling and antimicrobial properties of the resulting membranes. 

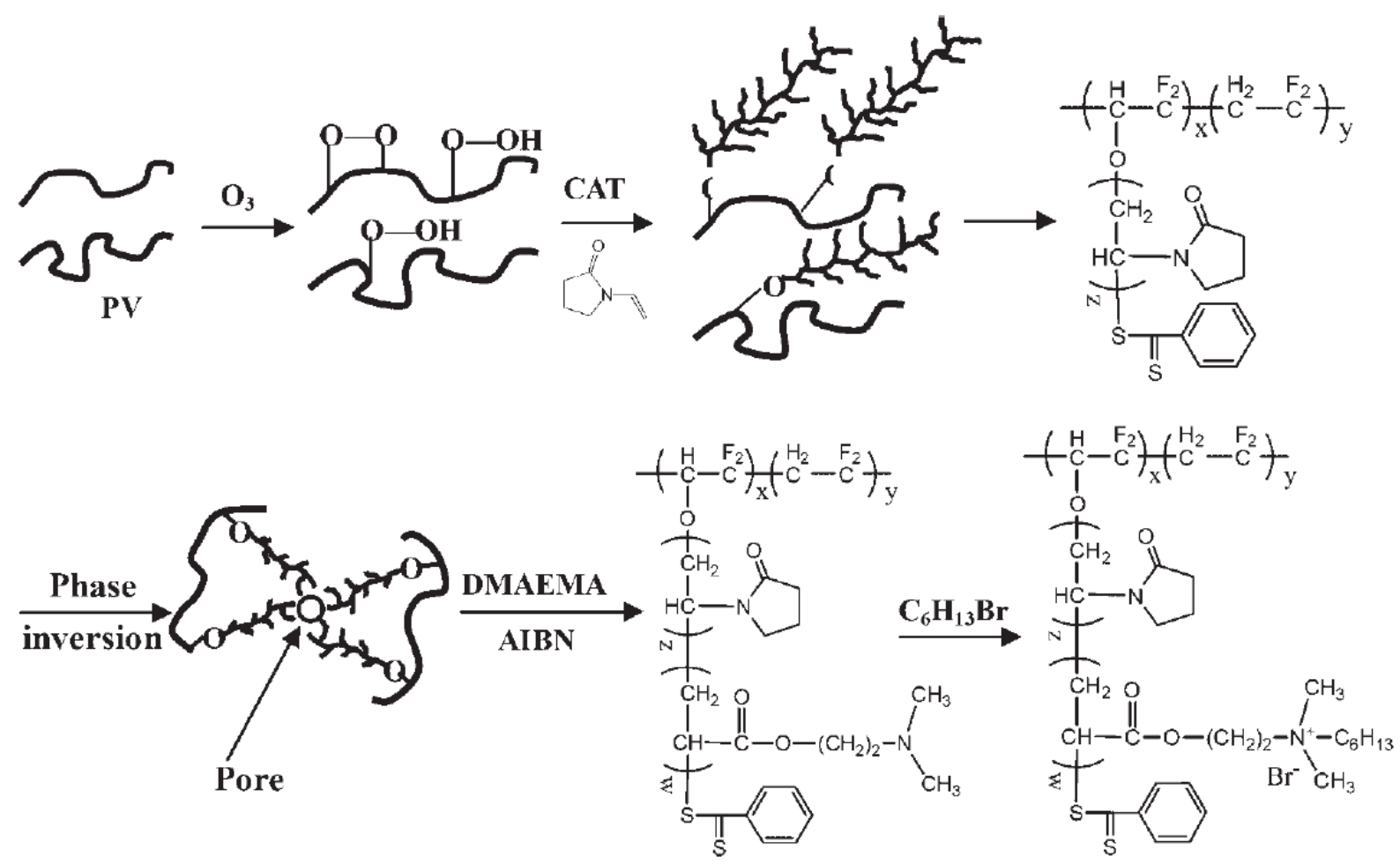

Scheme 47. Schematic illustration of the process of PNVP graft copolymerization with an ozone-preactivated PVDF backbone, by RAFT-mediated polymerization and the preparation of the PVDF-g-PNVP MF membrane by phase inversion.[195] Reproduced with permission from Wiley.

As displayed in schemes 6 and 24, PVDF-xanthate was synthesized via RAFT polymerization of VDF. Subsequently, the chemical modification of the xanthate end-group of the abovementioned PVDF into thiols via two strategies (aminolysis and elimination using sodium azide) was carried out. As reported by McKee et al.[196], the thiols were then immediately added onto the acrylate functionalities of 3-(acryloyloxy)-2-hydroxypropyl methacrylate via regioselective thia-Michael addition to form new PVDF-methacrylate macromonomers (Scheme 48). Results revealed that better coupling efficiency can be achieved via aminolysis 
protocol leading to well defined PVDF-methacrylate macromonomers. Thus synthesized PVDF-methacrylate was then copolymerized with methyl methacrylate (MMA), resulting in the complete conversion of the macromonomer and synthesis of novel PMMA-g-PVDF copolymers (Scheme 48).[197]

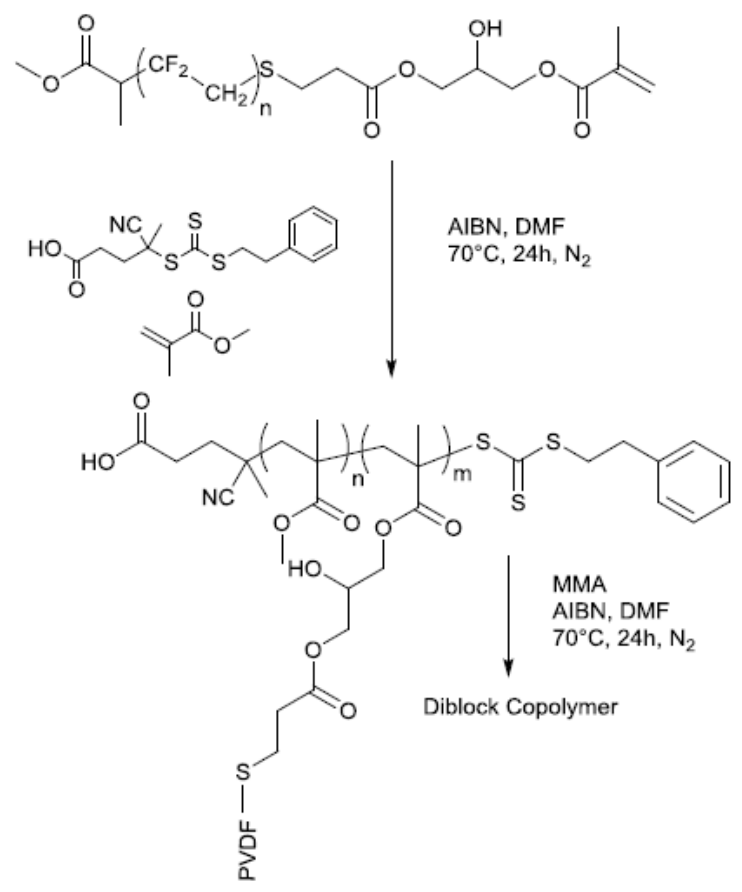

Scheme 48. RAFT copolymerization of a PDVF-methacrylate macromonomer with MMA (AIBN: azobisisobutyronitrile) to lead to PMMA-g-PVDF graft copolymer.[197] Reproduced with permission from Royal Society of Chemistry.

Chen et al.[198] reported the synthesis of PVDF-g-PEGMA graft copolymers via graft polymerization of OEGMA from the C-F bond of PVDF backbone via RAFT polymerization (Scheme 49). The pore size distribution of the resulting membranes prepared by RAFT polymerization was found to be much more uniform compared to that prepared via conventional radical polymerization process. Furthermore, the PVDF- $g$-PEGMA membranes 
displayed appreciablel resistance to $\gamma$-globulin fouling, in comparison to the neat hydrophobic PVDF microfiltration membranes.

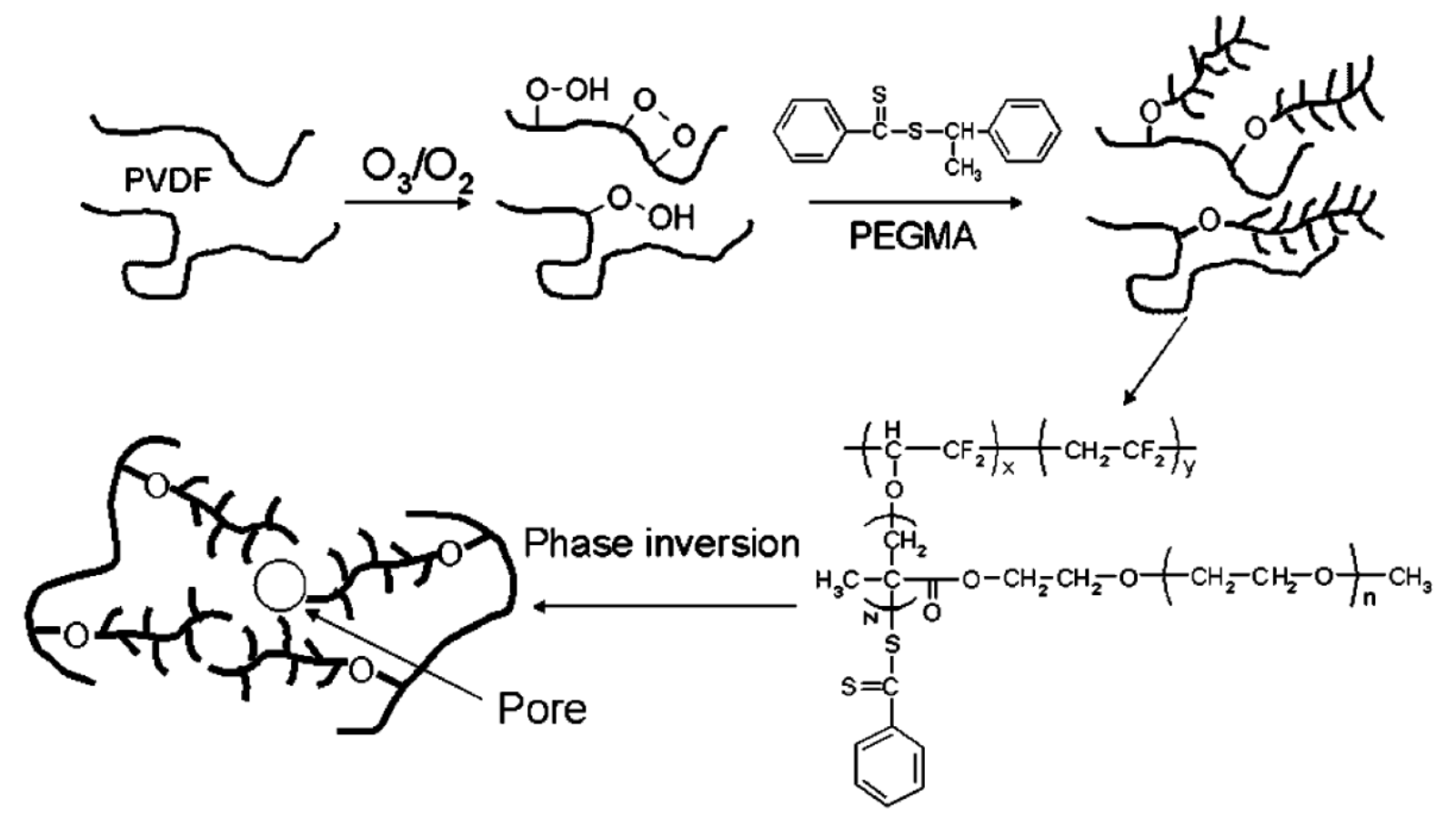

Scheme 49. Sketch of the processes to obtain PVDF-g-PEGMA copolymer and preparation of membrane by phase inversion.[198] Reproduced with permission from American Chemical Society.

\subsection{Graft copolymers via ATRP}

\subsubsection{From PVDF}

Mayes and coworkers comprehensively reported ATRP of M monomers from PVDF producing PVDF-based graft copolymers. In one example, Hester et al.[199] synthesized amphiphilic graft copolymers from commercial PVDF using ATRP. Surprisingly, because of strong C-F bond dissociation energy, the authors claimed the direct initiation of the secondary fluorinated site of PVDF facilitates the grafting of the hydrophilic comonomer (Figure 20). 
(a) Pure PVDF
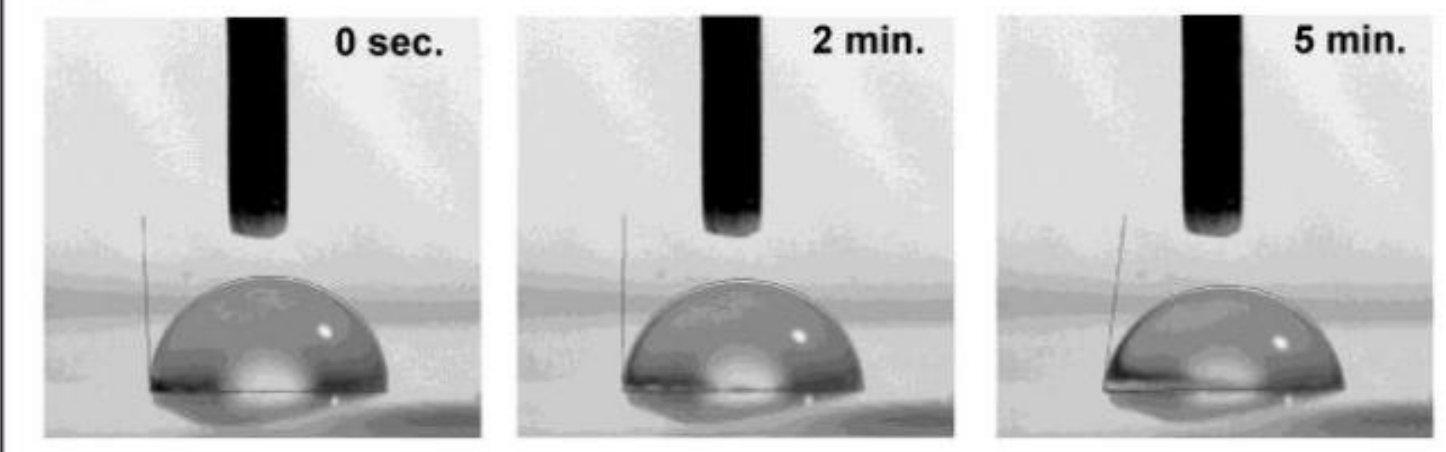

(b) $10 \mathrm{wt} \%$ PVDF-g-POEM
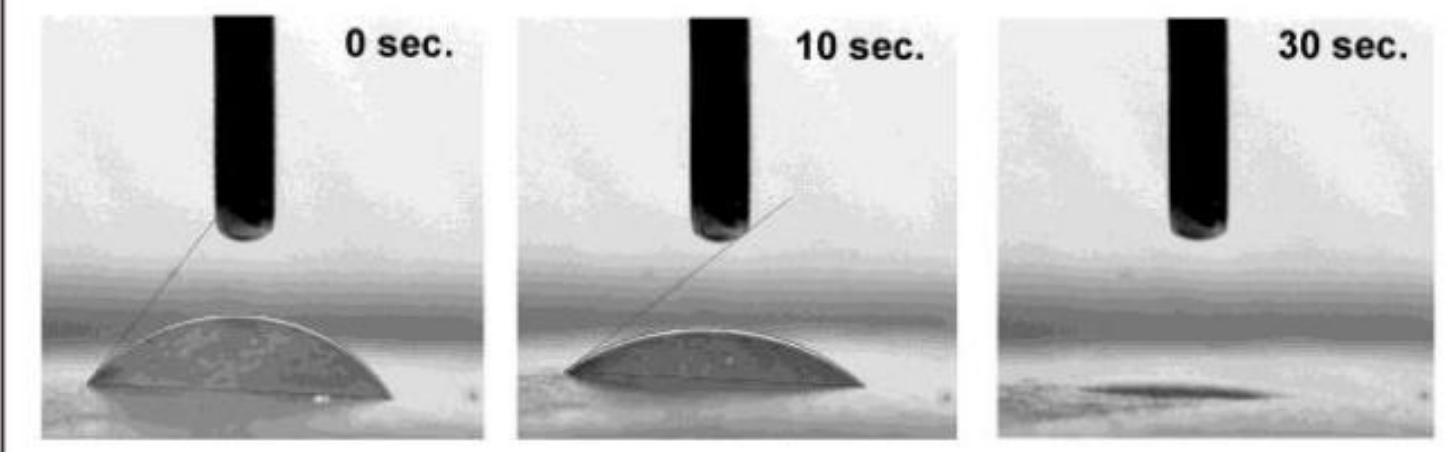

Figure 20. Images of water droplets at different times on (a) a pure PVDF membrane and (b) a membrane containing 10 wt \% PVDF- $g$-POEM.[199] Reproduced with permission from American Chemical Society.

The same team[199] prepared polymer blends consisting of PVDF and a free-radically synthesized amphiphilic comb polymer having a methacrylate backbone and PEO side chains, displaying fouling resistance. These authors[200] also produced polymer thin film composite membranes coated by amphiphilic graft copolymers which consisted of a PVDF backbone and POEM side chains. They also synthesized graft copolymers of VDF with MMA (PVDF- $g$ PMMA) or OEM (PVDF-g-POEM)[201] via "grafting from" method, using PVDF as 
macroinitiator for ATRP. Following that strategy, the same group[202] reported nanofiltration (NF) membranes prepared by coating a commercial PVDF ultrafiltration (UF) membrane support by a self-assembling graft copolymer. This graft PVDF-g-POEM polymer shows exceptional fouling resistance for a variety of model biofoulant solutions. Hester and Mayes[199] produced environmentally responsive membranes from polymer blends consisting of PVDF and a small proportion of an amphiphilic comb polymer having a PVDF backbone and PMAA side chains. The comb polymer was synthesized by an ATRP technique. Akthakul et al.[203] generated noncircular pores on the surface of asymmetric polymer membrane and confirmed by spinodal demixing. The same team[204] demonstrated the effects of process and solution parameters on the permeability and selectivity of PVDF- $g$-POEM thin film composite (TFC) NF membranes. By controlling the degree of swelling of the hydrated PEO chains filling the channels, the effective pore size of the membranes is shown to be tunable. Membrane permeability to water and to organic dye molecules of approximately $1 \mathrm{~nm}$ diameter was found to increase when the solvent quality of the feed for the PEO chains was reduced by raising the temperature, pressure, or ionic strength, or by the addition of ethanol. Their study explored the influence of process parameters on the permeability and selectivity of PVDF- $g$-POEM thin film composite NF membranes (Figure 21). These developed membranes have potential applications in biochemical, pharmaceutical, and food industries for low-cost, high-throughput fractionation of molecules. 


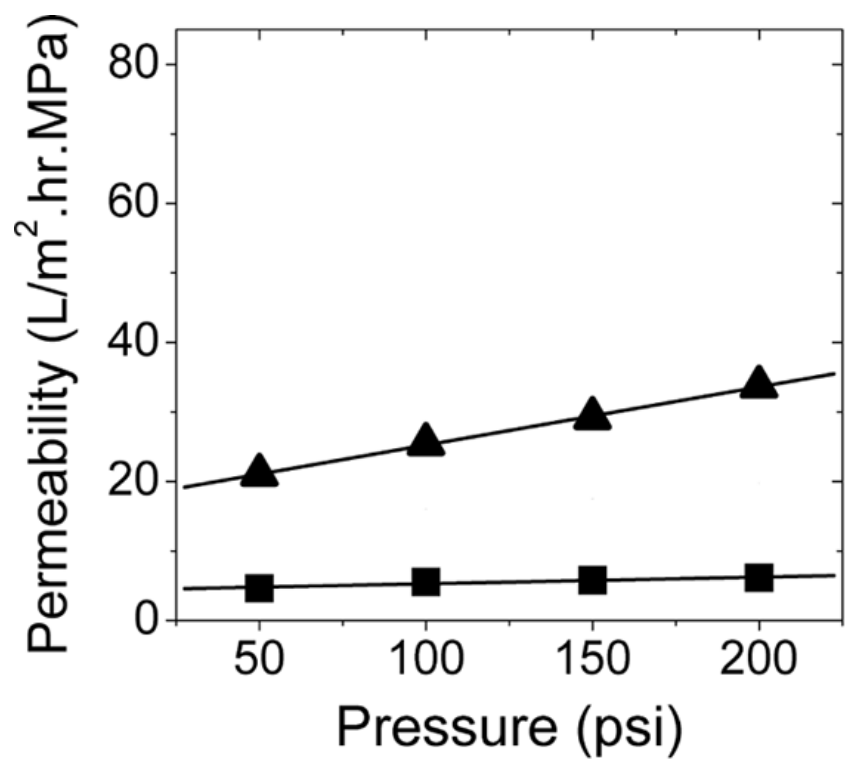

(a)

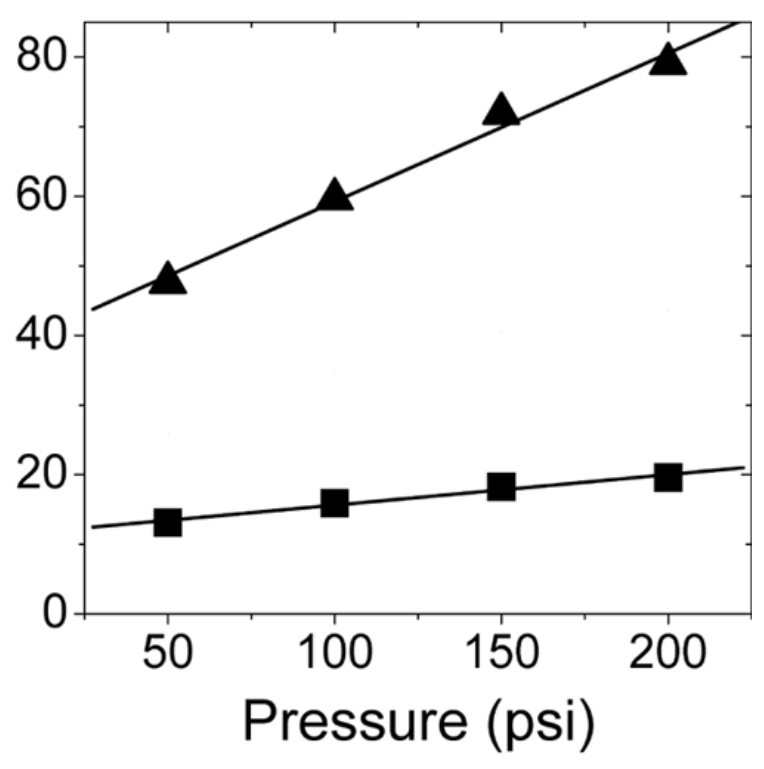

(b)

Figure 21. Permeability of PVDF- $g$-POEM nanofiltration membrane with a feed of (a) deionized water and (b) $0.2 \mathrm{M} \mathrm{NaCl}$, at room temperature (rectangle) and at $70{ }^{\circ} \mathrm{C}$ (triangle) at varying pressures.[204] Reproduced with permission from Taylor \& Francis.

Zhang et al.[205] synthesized a series of PVDF-g-PSSA graft copolymers via ATRP and then extensively studied how the polymer microstructure (backbone molar mass, grafting density, graft length), electrochemical properties (sulfonic acid concentration, ion exchange capacity, etc.) affects their morphology, water uptake, and proton conductivity under various environmental conditions (temperature and relative humidity).

Lee et al.[206] prepared PVDF- $g$-PtBA graft copolymers via grafting tBA from PVDF using ATRP technique (Figure 22). Though no clear evidence of grafting was mentioned (absence of SEC chromatogram and ${ }^{19} \mathrm{~F}$ NMR spectrum), the resulting copolymer demonstrated dielectric constant control and resulted in marked increase in the output power. 


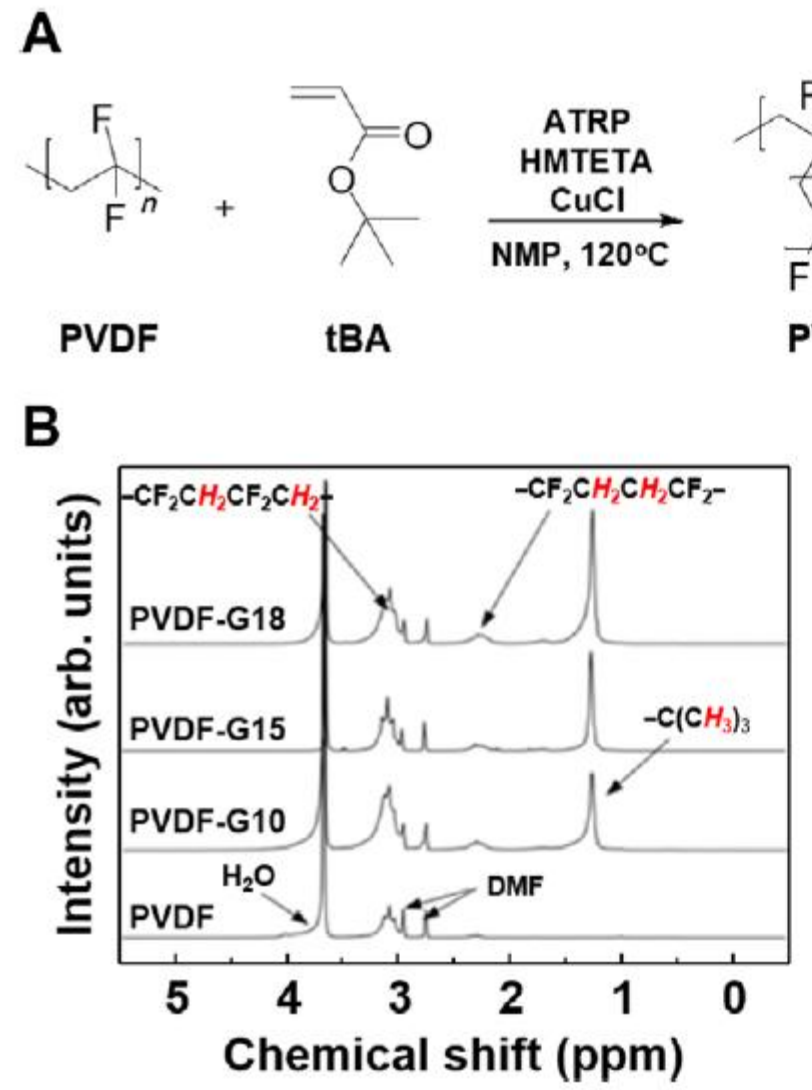

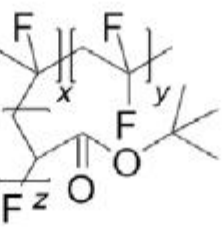

PVDF-Gn
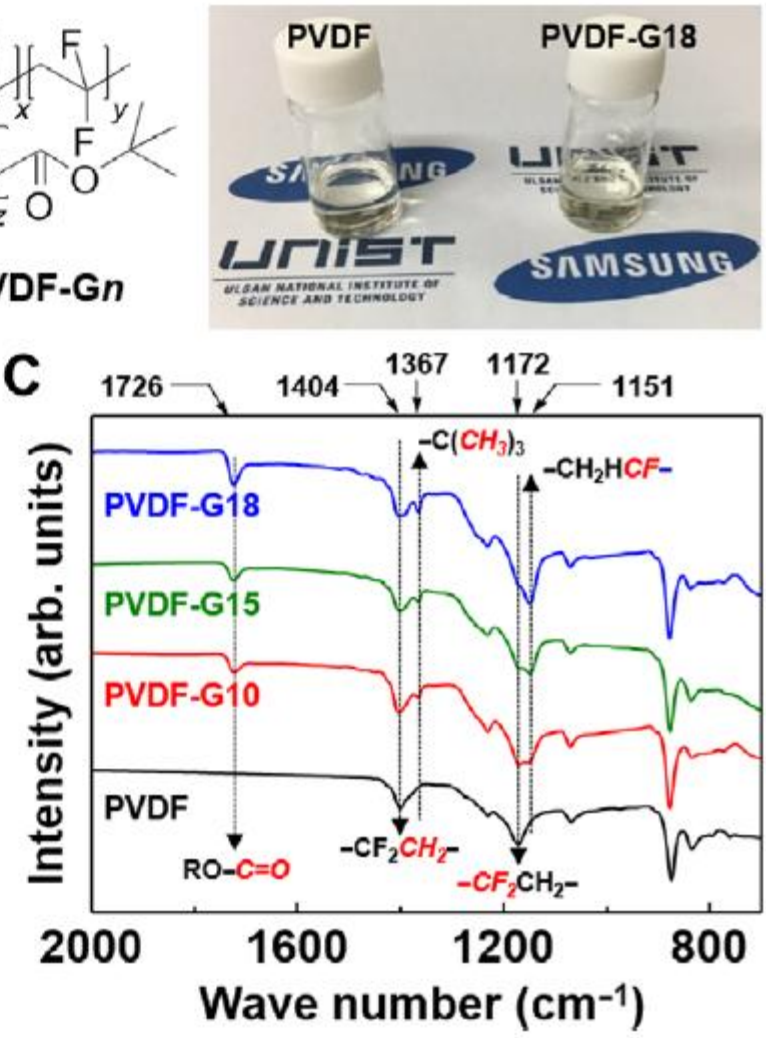

Figure 22. (A) Synthesis of PVDF- $g$-PtBA graft copolymers and photograph of the PVDF and PVDF-g-PtBA graft copolymers NMP solutions. 1H NMR (B) and FTIR (C) of PVDF and PVDF-g-PtBA graft copolymers.[206] Reproduced with permission from Science Publishing Group.

From the same strategy, Nandi's group[207] reported the grafting of DMAEMA in solution phase directly from PVDF backbone. The graft length was the same for different polymerization times, but grafting density increased with increasing polymerization time. For the first time, the same group[208] developed a new "grafting from" strategy of different monomers onto the PVDF backbone via atom transfer radical coupling (ATRC) and ATRP (Scheme 50). 

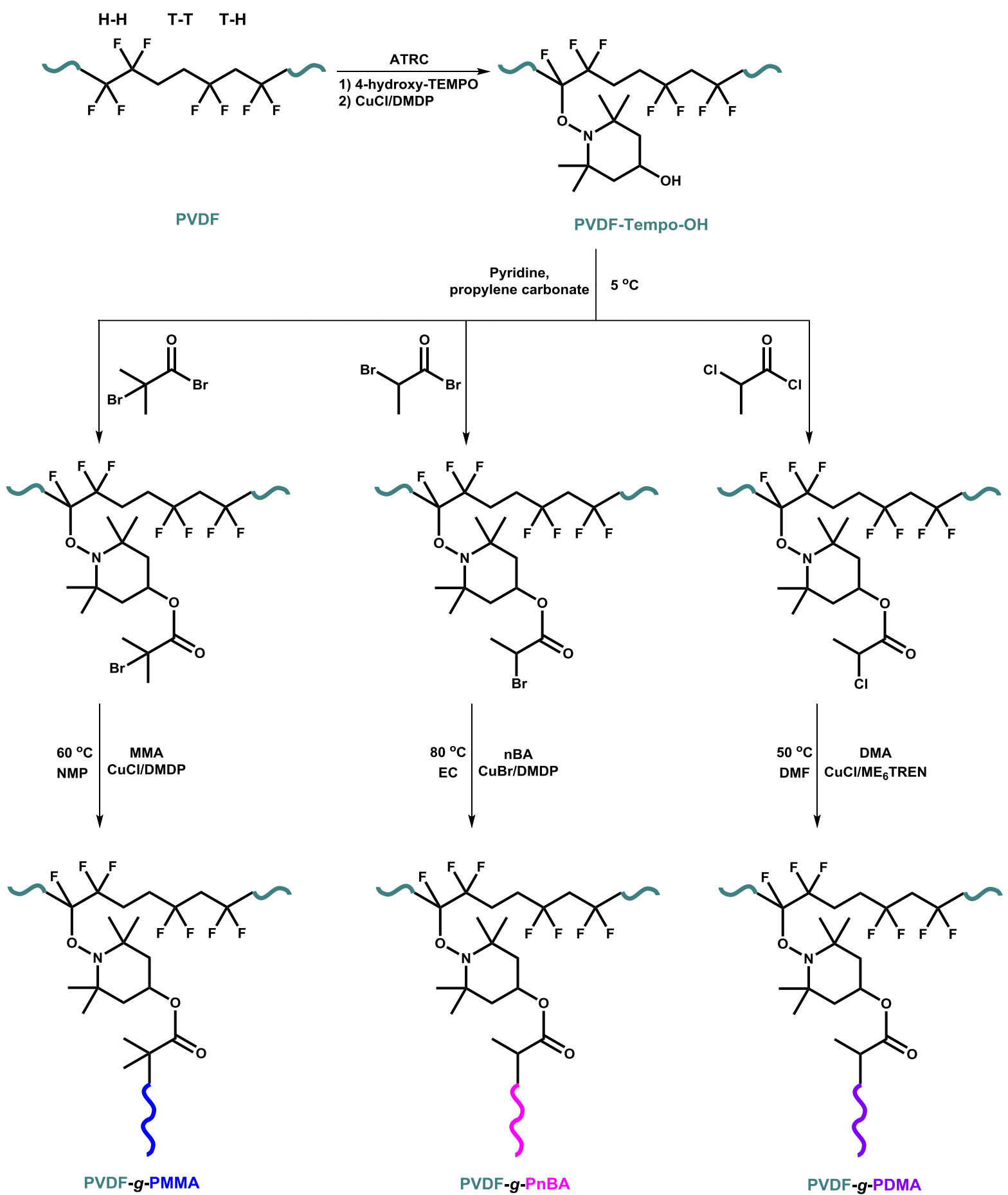

Scheme 50. Synthetic scheme of different PVDF graft copolymers using coupled atom transfer radical coupling (ATRC) and ATRP approach,[208] where NMP, MMA, nBA, DMA, EC and DMF stand for N-methylpyrrolidone, methyl methacrylate, butyl acrylate, N,N-dimethyl 
acrylamide, ethylene carbonate and N,N-dimethylformamide, respectively. Reproduced with permission from Wiley.

The same team recently published an interesting review article on this topic.[209] In order to tune the properties of PVDF and introduce multifunctionality such as to make permeation of water molecules through the PVDF based membranes, different hydrophilic polymers like PEOM, poly(methacrylic acid) and PDMAEMA were grafted from PVDF backbone via ATRP (Scheme 51). Thus, prepared water-soluble graft copolymer shows excellent mechanical and adhesive properties. This approach resulted in interesting solution properties, ion and electron conducting PVDF which were useful as antifouling membranes, super glues and super tough materials.

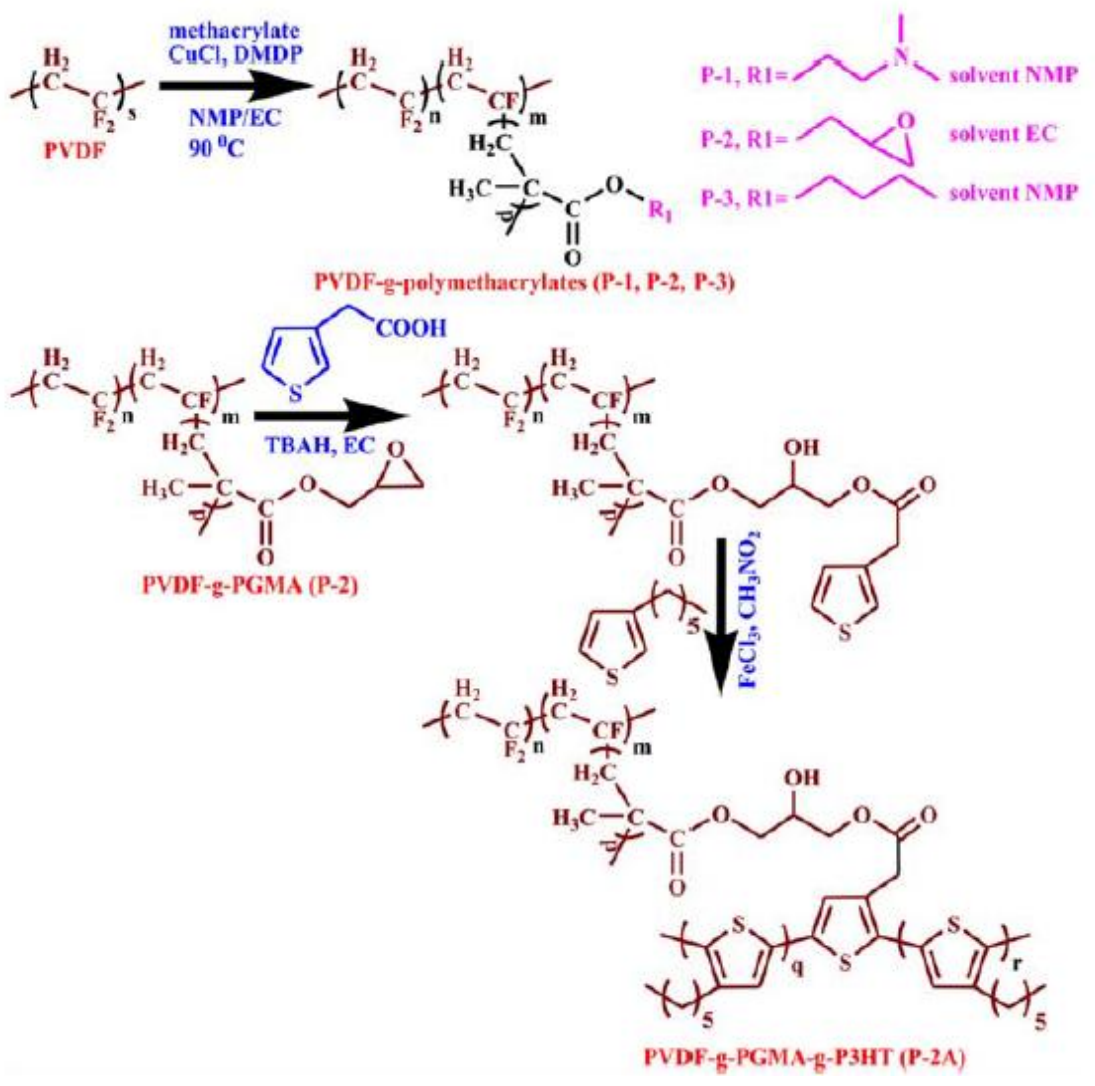

Scheme 51. Synthesis of P-1, P-2, P-3 PVDF- $g$-poly(M) graft copolymers using ATRP and 
double grafting of P-2 by oxidative polymerization from PVDF.[209] Reproduced with permission from Wiley.

He et al.[210] prepared original graft copolymers by grafting arginine-glycine-aspartic acid (RGD) peptide-click-poly(glycidyl methacrylate) (PGMA) polymer brushes onto the PVDF surface by the combination of surface-initiated ATRP and click reaction (Figure 23). This led to improved chemical and mechanical properties of PVDF films and enhancement of the cytocompatibility of PVDF films. Thus synthesized PVDF films are potentially useful for biomedical and tissue engineering applications.

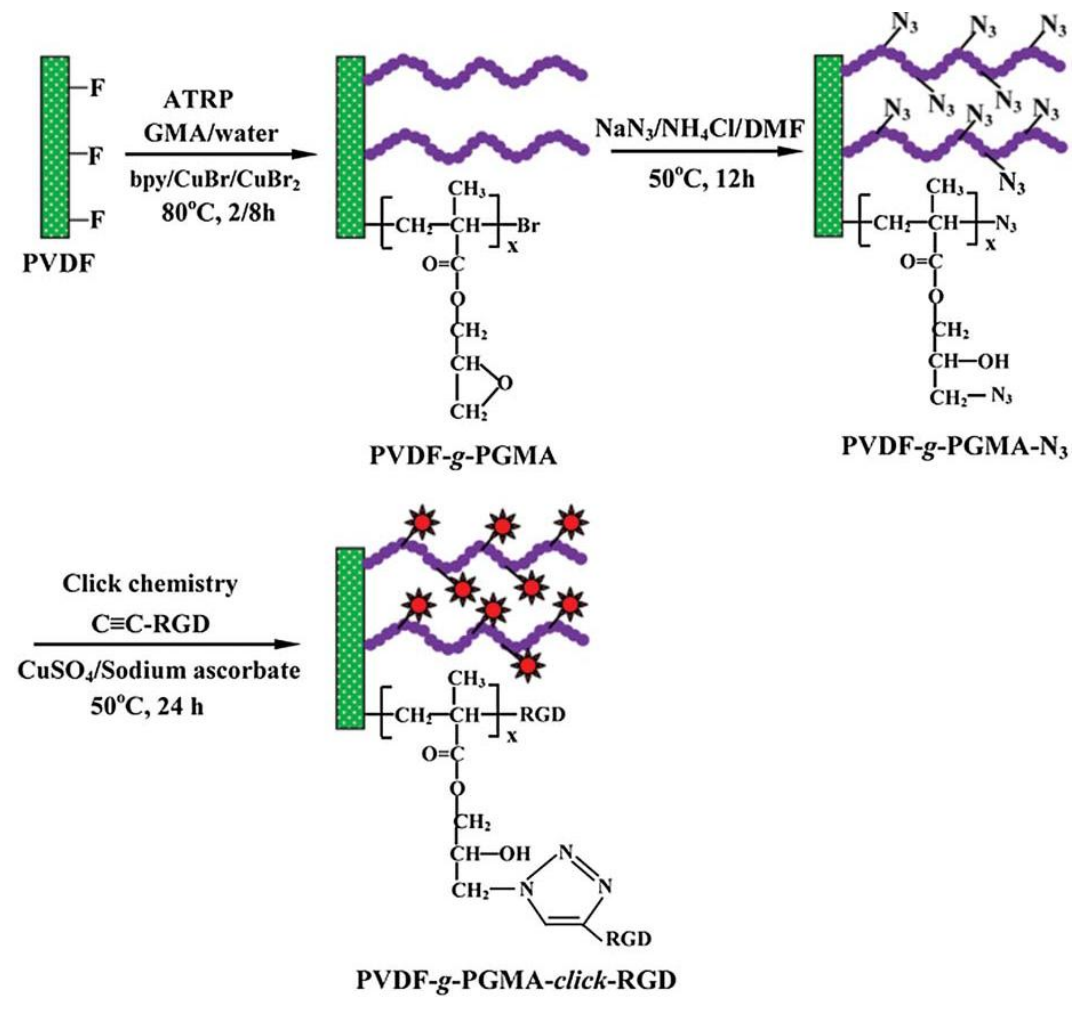

Figure 23. Schematic illustration of the synthesis of PVDF-g-PGMA surface, subsequent introduction of azide groups to produce the PVDF-g-PGMA-N ${ }_{3}$ surface, and the conjugation of RGD peptide to produce PVDF-g-PGMA-click-RGD surface, where GMA and RGD stand for glycidyl methacrylate and arginine-glycine-aspartic acid, respectively.[210] Reproduced with 
permission from Royal Society of Chemistry.

Holmberg et al.[211] prepared PVDF-g-PVBC graft copolymers via ATRP from C-X bonds of PVDF. Then, they grafted styrene from the C-Cl bonds of the PVDF- $g$-PVBC graft copolymers (Scheme 52). Thus prepared novel membranes have potential fuel-cell applications.
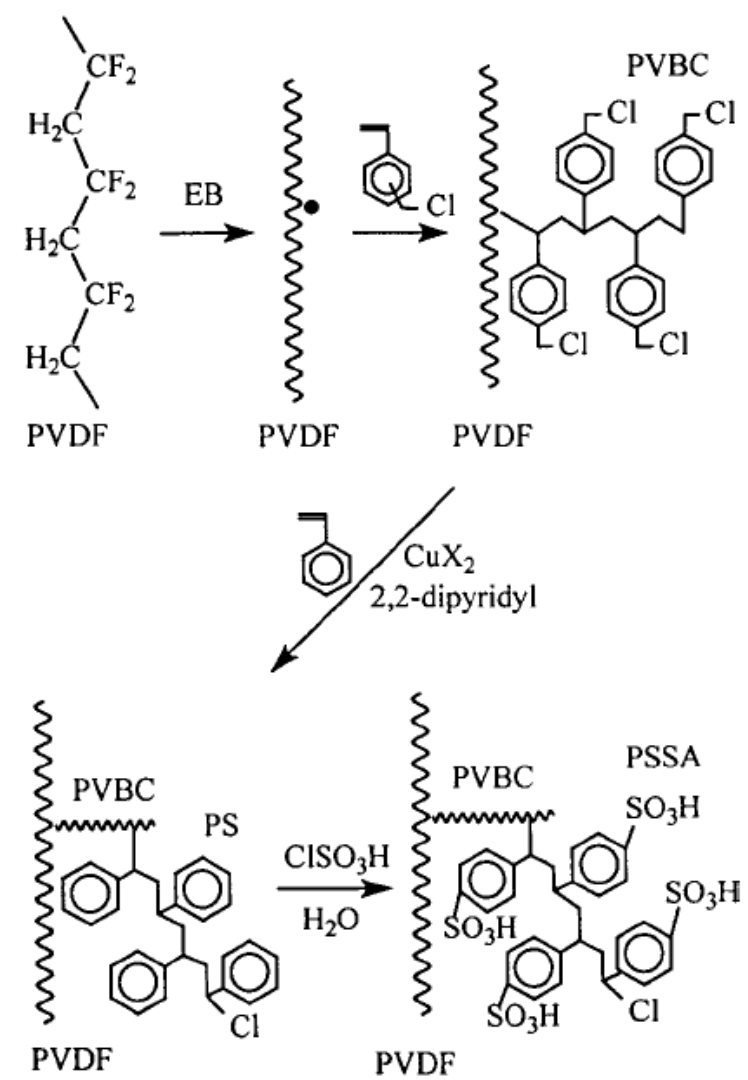

Scheme 52. Synthesis of sulfonated PVDF- $g$-PVBC-g-PS proton-conducting membranes.[211] Reproduced with permission from Wiley.

To the best of our knowledge, only one example describes the preparation of graft copolymers from a fluorinated macroinitiator containing a $\mathrm{C}$ - $\mathrm{Br}$ cleavable bond. Indeed, the chemical modification of poly(VDF-co-BDFO) copolymers,[212] synthesized by conventional radical 
copolymerization of VDF with 8-bromo-1H,1H,2H-perfluorooct-1-ene (BDFO),[213] was successfully achieved by ATRP of styrene catalyzed by $\mathrm{CuBr}$ and $1,1,4,7,10,10$ hexamethyltriethylenetetramine (HMTETA) as the ligand (Scheme 53). The linear molar masses of such PVDF-g-PS graft copolymers versus the styrene conversion relationship concomitant with low dispersities (starting from 1.8 for poly(VDF-co-BDFO) random copolymers) evidenced a controlled tendency of that reaction.<smiles>CC(C)(C)CCCCCC(CC(C)(C)C)C(C)(C)C</smiles>

poly(VDF-co-BDFO)

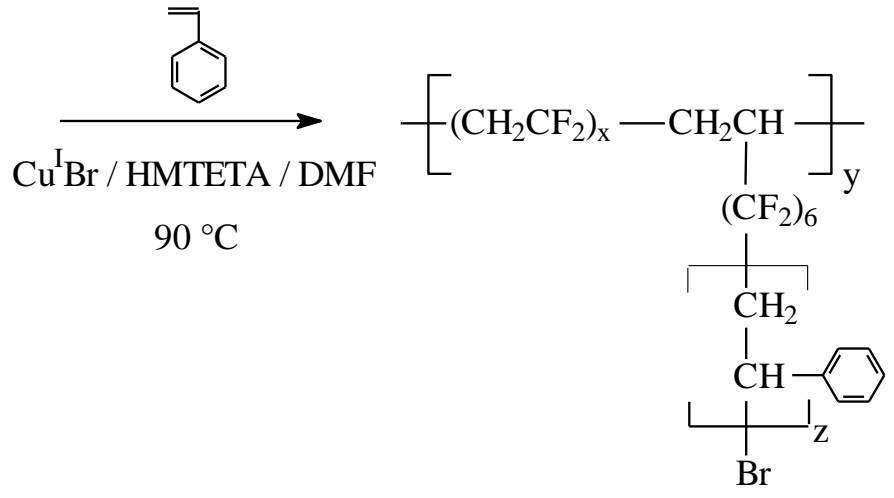

PVDF-g-PS

Scheme 53. PVDF- $g$-PS graft copolymers produced by ATRP of styrene with a poly(VDF-coBDFO) macroinitiator. BDFO, HMTETA, and DMF stand for 8-bromo-1H,1H,2H-perfluorooct1-ene, 1,1,4,7,10,10-hexamethyltriethylenetetramine, and dimethylformamide, respectively.[212] Reproduced with permission from American Chemical Society.

Besides ATRP strategy, Zhang et al.[214] reported the grafting of PMMA onto PVDF by single electron transfer-controlled radical polymerization (SET-CRP) toward C-F bonds catalyzed with $\mathrm{Cu}^{0}$ and bipyridine (bpy). In the absence of halogen exchange, $\mathrm{Cu}^{0}$ showed significantly improved catalytic activity than $\mathrm{Cu}^{\mathrm{I}}$ complexes based typical ATRP system.

\subsubsection{From CTFE-based copolymers}

Many teams have reported the synthesis of graft copolymer via ATRP of various vinyl monomers such as styrene and (meth)acrylates from CTFE-containing copolymers.[145] The 
authors claimed that the grafting occurred through cleavage s' of the $\mathrm{C}-\mathrm{Cl}$ bond of CTFE, although they were unable to provide any direct evidence. Indeed, simple ${ }^{19} \mathrm{~F}$ NMR or XPS analyses would evidence the shift of signal assigned to - $\mathrm{CFCl}$ - to $-\mathrm{CF}\left[\mathrm{CH}_{2} \mathrm{CRR}^{\prime}\right]-$. Applications varied from coatings to various specific membranes such as water treatment (or desalination) or antifouling, as well as alkaline and protonic fuel cell membranes.

6.4.3. From poly(VDF-co-CTFE) copolymers via the cleavage of $\mathrm{C}-\mathrm{Cl}$ bond in CTFE units Zhang and Russell[215] pioneered the synthesis of poly(VDF-co-CTFE) based graft copolymers via grafting of polystyrene (PS) and poly(tert-butyl acrylate) (PtBA) from poly(VDF-co-CTFE) via ATRP. Inspired their work, Guan et al.[216, 217] synthesized poly(VDF-co-CTFE)-g-PSt copolymers and studied their confined ferroelectric properties for electric energy storage applications. Simultaneously, Tan et al.[218] carried out a detailed study on the development of a novel method to determine the competition between chain transfer reaction and initiation reaction of free radicals by analyzing the structure of grafting copolymers from poly(VDF-coCTFE). Their results indicate that the method may help to understand the chain transfer reaction in ATRP as well as its influence on the chain end of resultant polymer.

The same year, Seo et al.[219] prepared amphiphilic poly(VDF-co-CTFE)-g-poly(HEA-co-SSA) graft copolymer via ATRP by grafting crosslinkable chains of poly(2-hydroxyl ethyl acrylate), PHEA, and proton conducting chains via PSSA grafts (Scheme 54). The resulting membranes also exhibited a maximum proton conductivity of $0.062 \mathrm{~S} / \mathrm{cm}$ at $6 \mathrm{wt} \%$ of SA concentration, resulting from competitive effect between the increase of ionic groups and the degree of crosslinking. XRD patterns also revealed that the crystalline structures of $\mathrm{P}(\mathrm{VDF}-$-co-CTFE) copolymers disrupted upon graft polymerization and crosslinking. These membranes exhibited good thermal stability at least up to $250{ }^{\circ} \mathrm{C}$. 


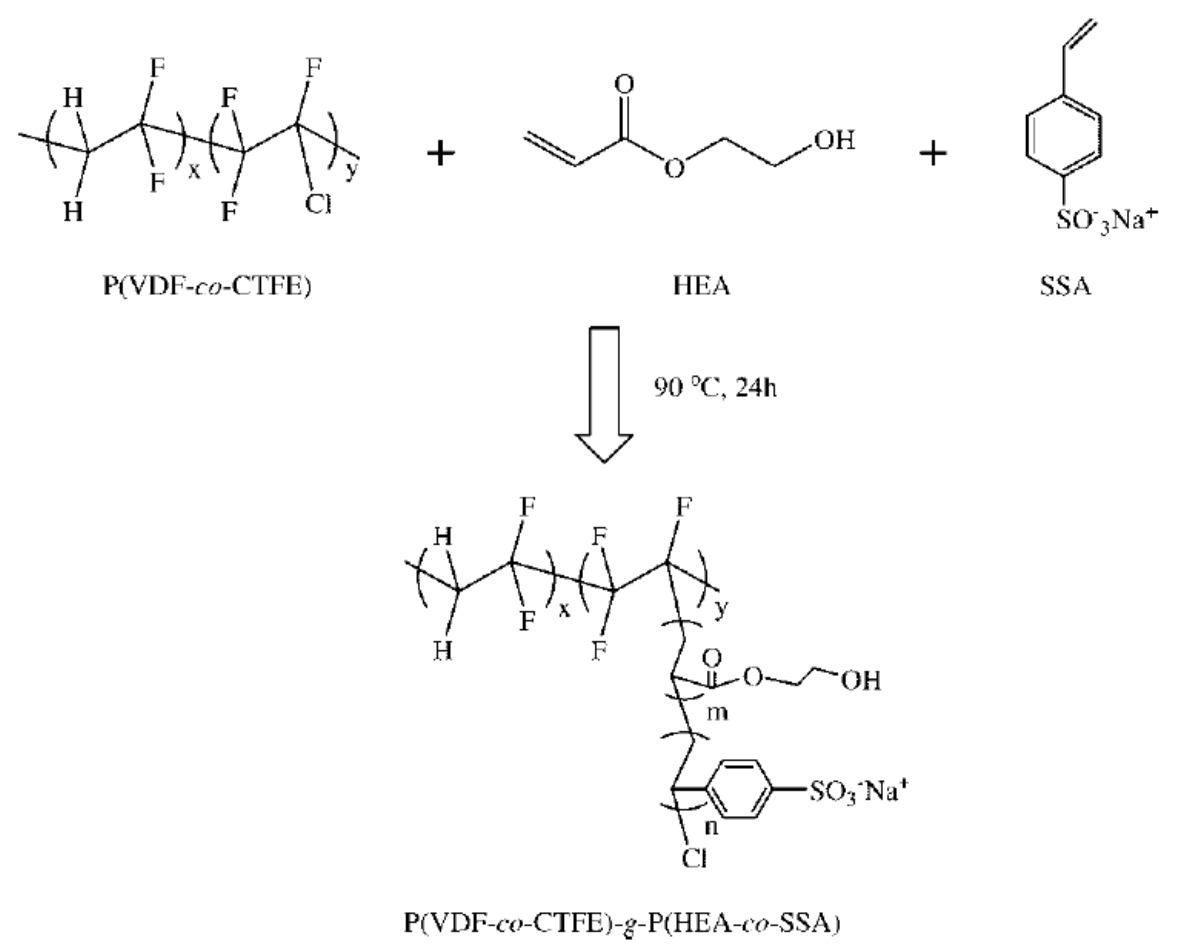

Scheme 54. Atom transfer radical copolymerization of HEA and SSA from poly(VDF-coCTFE) for the synthesis of $\mathrm{P}(\mathrm{VDF}-c o-\mathrm{CTFE})-\mathrm{g}$-P(HEA-co-SSA) graft copolymer.[219] Reproduced with permission from Wiley.

$\mathrm{Hu}$ et al.[220] reported the synthesis of functional fluoromaterials such as poly(VDF- co- CTFE)- $g$ - PAN through poly(VDF- $c o$ - CTFE) macroinitiator using acrylonitrile (AN) and trace amount of $\mathrm{Cu}(0) /$ tris(2(dimethylamino)ethyl)amine $\left(\mathrm{Me}_{6}-\mathrm{TREN}\right)$ in DMSO at ambient temperature. The same group[221] developed a series of 2,6- bis(imino)pyridines for grafting of PMMA from poly(VDF- co- CTFE). The authors also investigated the relationship between the chemical structure of 2,6-bis(imino)pyridine ligands and the catalytic activity.

Later on, Zhu et al.[222] developed a green, highly effective and energy-saving route for grafting of MMA and AN from poly(VDF- co- CTFE). The modified poly(VDF-co-CTFE)-g-PMMA 
and poly(VDF-co-CTFE)-g-PAN have potential applications as dielectric materials. Wang et al.[223] developed a linear-like PVDF-based polymer dielectric via grafting PMMA onto main chains of poly(VDF-ter-TrFE-ter-CTFE) terpolymer via ATRP (Figure 24). PMMA grafted terpolymer poly(VDF-ter-TrFE-ter-CTFE)-g-PMMA) shows linear-like dielectric characteristics along with enhanced discharging efficiency of $\sim 80 \%$ under $250 \mathrm{MV} / \mathrm{m}$, which is over $100 \%$ higher than that of pristine terpolymer. The above strategy might be useful for making composite dielectrics bearing simultaneously high energy density and high discharging efficiency.

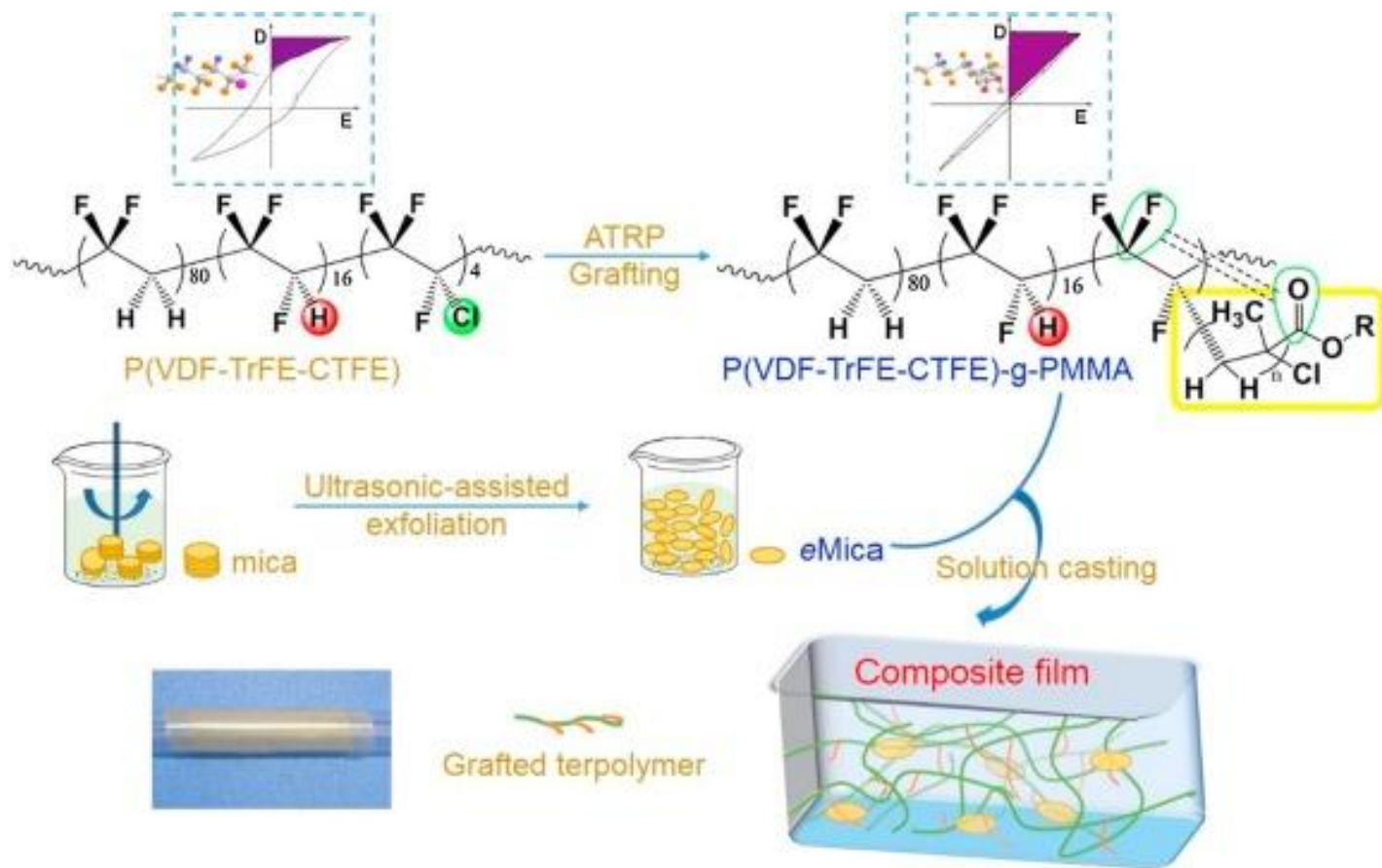

Figure 24. Schematic illustration for preparation of polymer/eMica nanocomposite films.[223] Reproduced with permission from Elsevier.

Though fluorinated block copolymers have been claimed without any dielectric properties supplied[124], the electroactive features of poly(VDF-ter-TrFE-ter-CTFE)- $g$-polystyrene or poly(VDF-ter-TrFE-ter-CTFE)-g-PMMA graft terpolymers [286], prepared by ATRP from poly(VDF-ter-TrFE-ter-CTFE) macroinitiators, were deeply examined by NMR spectroscopy 
(Scheme 55).

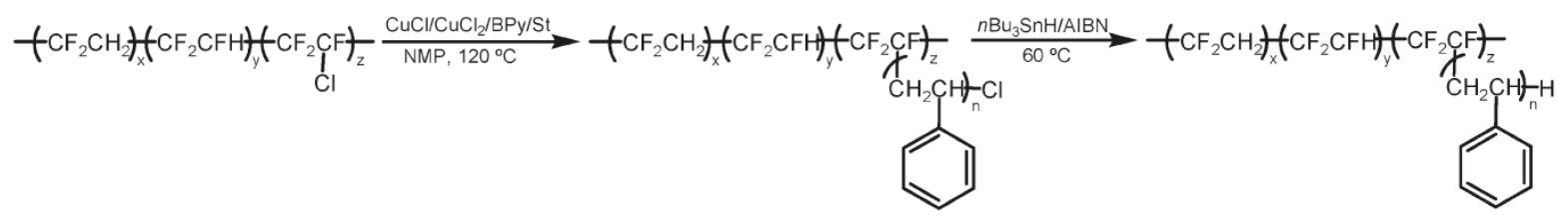

Scheme 55. Preparation of poly(VDF-ter-TrFE-ter-CTFE)-g-PS graft copolymer by ATRP of styrene, followed by a reduction step to avoid thermal cross-linking during melt processing.[217] Reproduced with permission from American Chemical Society.

Figure 25 nicely evidences the presence of the characteristic signals centered at ca. $6.5-7.2 \mathrm{pm}$ assigned to PS grafts while those assigned to CTFE units at -109 and -121 ppm were affected in the ${ }^{1} \mathrm{H}$ NMR and ${ }^{19} \mathrm{~F}$ spectra, respectively. However, main issue of this ATRP deals with the use of copper complex that must be eliminated from the terpolymer to avoid any presence of metallic ions in the resulting films which would interfere in their electroactive properties. Copper ligands are regarded as weak bases that can trigger possible dehydrofluorination of VDF and TrFE units or dehydrochlorination of CTFE ones, inducing double bonds all along the terpolymer chain. Wang and Zhu's groups overcame that limitation by a post-treatment with $\mathrm{n}-\mathrm{Bu}_{3} \mathrm{SnH}$ that favored the removal of pendant $\mathrm{Cl}$ atoms (Scheme 55) and to avoid any cross-linking and side reactions of the graft copolymers during annealing and further thermal treatments, in addition to also modify unreacted CTFE units into TrFE.[216, 217, 224] 

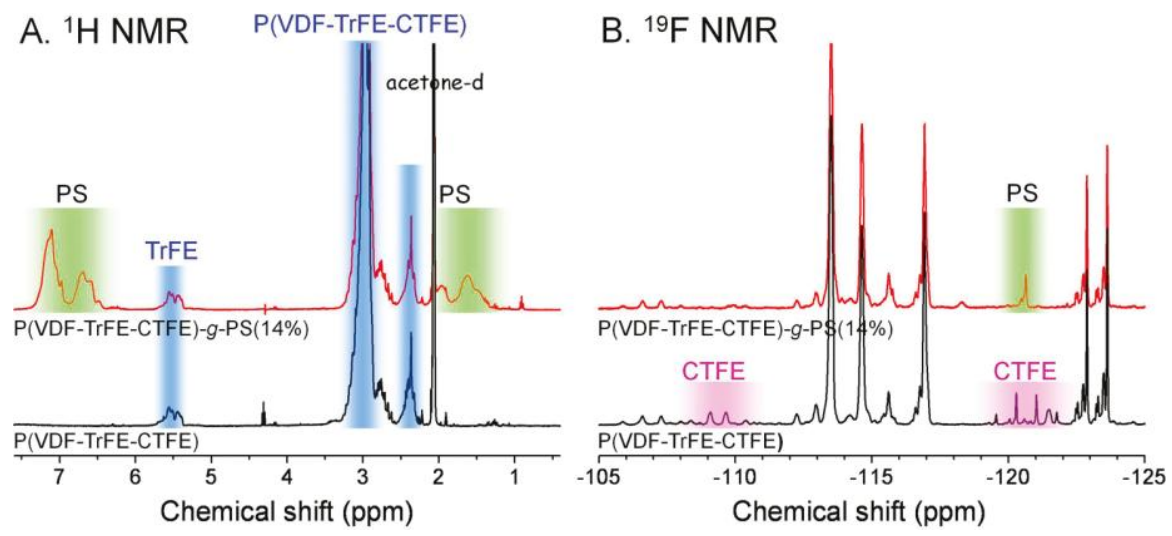

Figure 25. (A) ${ }^{1} \mathrm{H}$ and (B) ${ }^{19} \mathrm{~F}$ NMR spectra of poly(VDF-ter-TrFE-ter-CTFE) 88.8/7.7/3.5 and poly(VDF-ter-TrFE-ter-CTFE)-g-PS (14 mol \%), respectively. In the ${ }^{1} \mathrm{H}$ NMR spectra in (A), PS and poly(VDF-ter-TrFE-ter-CTFE) peaks are highlighted with green and blue colors. In the ${ }^{19} \mathrm{~F}$ NMR spectra in (B), -CFCl- fluorine atoms are highlighted with magenta color and fluorine atoms adjacent to PS side chains are highlighted with green color.[217] Reproduced with permission from American Chemical Society.

Grafted copolymers display disordered crystal phases due to many grafting sites and broad dispersity of the grafted chains. The crystallization in the $\beta$ phase $(6.5 \%)$ in poly(VDF-ter-TrFEter-CTFE)-g-PS (14 mol \% of PS) was lower than that in 93/7 mol \% poly(VDF-co-TrFE) copolymer $(17.6 \%)$ because the preferential all-trans conformation was affected by the PS side chains linked to the poly(VDF-ter-TrFE-ter-CTFE) main chain.[217] The overall crystallinity was reduced, and thus strongly disturbed the electroactive property.

A similar approach was developed by Li et al.[225] using ATRP of ethyl methacrylate (EMA) for antiferroelectric materials.[225, 226] Contrarily to the bad compatibility and the poor intermolecular forces between PVDF and PS chains, PEMA side chains were homogeneously distributed in the amorphous phase as shown in Figure 26. 

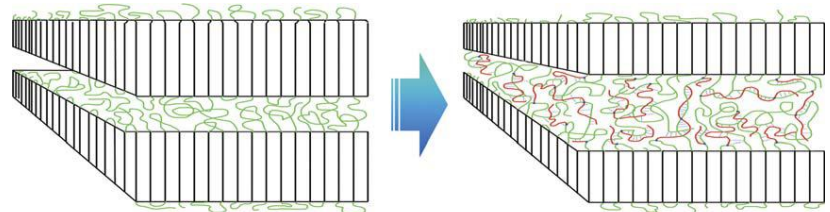

$\Pi \Pi \Pi \pi$ Crystal $\sim$ Amorphous $\sim$ PEMA

Figure 26. Sketch of the influence of PEMA grafting from ATRP of EMA from a poly(VDF-terTrFE-ter-CTFE) terpolymer resulting in an antiferroelectric material.[227] Reproduced with permission from Royal Society of Chemistry.

The presence of PEMA side chains was claimed to reduce the growing of large crystals. This property was evidenced by DSC (the melting temperature slightly decreased from 141 to $137^{\circ} \mathrm{C}$ while the crystallinity decreased from 24.5 to $8.9 \%$ as the PEMA content increased from 0 to 26 wt \%). These results suggest that the PEMA chain could also effectively impede the ferroelectric crystal domain nucleation and growth due to the complete miscibility of PEMA and PVDF.[225] Tsang et al.[228] reported the synthesis and characterization of graft and diblock ionic copolymers both containing fluorinated PSSA segments (Figure 27). These series were designed to possess similar compositions but considerably different architectures, the nature of which will be shown to significantly affect the morphology of the membranes and thus their respective conductive properties. Actually, the authors showed that the graft copolymers had a better segregation that enabled to increase the proton conductivity (1.0 to $1.2 \mathrm{mmol} / \mathrm{g})$ of the resulting membranes. However, no data was mentioned on the possible competitive presence of $\mathrm{Cl}$ atoms in unreacted CTFE units, that should favor the film forming properties, and $\mathrm{CF}_{3}$ groups in $\mathrm{HFP}$ units. 


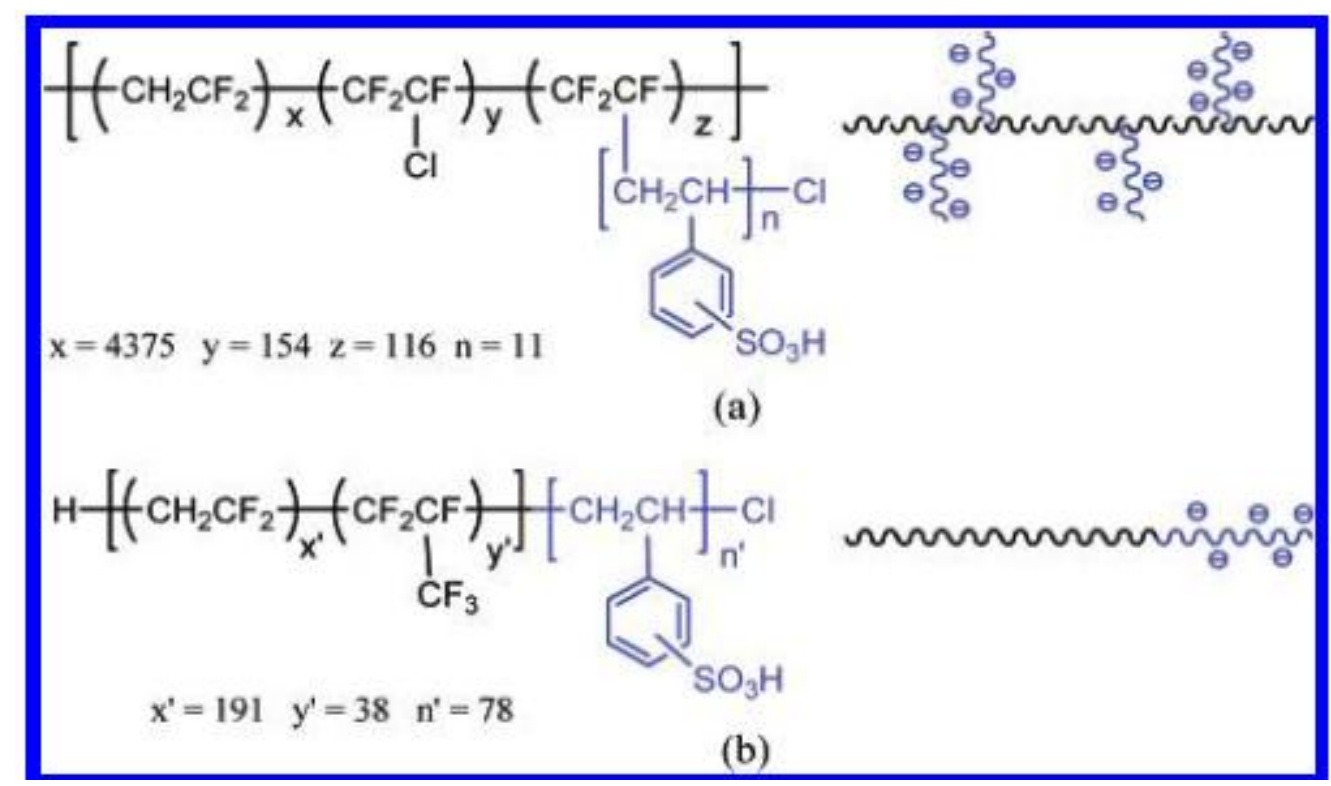

Figure 27. Chemical structures of (a) poly(VDF-co-CTFE)-g-PSSA graft and (b) poly(VDF-coHFP)- $b$-PSSA diblock copolymers.[228] Reproduced with permission from American Chemical Society.

Koh et al.[229] synthesized graft copolymers consisting of poly(VDF-co-CTFE) backbone and PSSA side chains by ATRP for composite nanofiltration (NF) membranes. They noted that both the rejections and the flux of composite membranes increased with increasing PSSA concentration in the copolymer. This feature might be due to the increase in $\mathrm{SO}_{3} \mathrm{H}$ groups and membrane hydrophilicity, as supported by contact angle measurements. The same group[230] also developed an amphiphilic comb polymer via grafting of OEM from the chlorine functionalities of poly(VDF-co-CTFE) copolymers through ATRP to process ultrafiltration (UF) membrane. The mechanism of initiation was confirmed by ${ }^{1} \mathrm{H}$ NMR and FTIR spectroscopy. The antifouling properties of the membranes was considerably improved compared to those of the poly(VDF-co-CTFE) copolymers. 
Kim et al.[231] later developed poly(CTFE-co-VDF)- $g$-poly(SSA) graft copolymers by surface initiated ATRP, using 4-styrene sulfonic acid (SSA) as the monomer, instead of postpolymerization modification of styrene to SSA. These membranes are also used for proton conducting copolymers.[231, 232] For the same application, Yang et al.[233] reported poly(VDF-co-CTFE)-g-PS graft copolymers to gain insight into the role of crystallinity and morphology in proton transport through solid polymer electrolytes, consisting of a hydrophobic, fluorous backbone and poly(styrenic) graft chain of varied length.

In addition, Park et al.[234] synthesized original poly(CTFE-co-VDF)- $g$-PSSA copolymers via surface-initiated ATRP of styrene from CTFE, followed by a sulfonation. Thus prepared copolymers were then mixed with $\mathrm{TiO}_{2}$ to prepare a hybrid proton-conducting membrane for use in high temperature fuel cells. These membranes demonstrated excellent thermal stability up to $280^{\circ} \mathrm{C}$.

\subsubsection{From poly(VDF-co-HFP) copolymers}

Hwangbo et al.[235] described the synthesis of graft copolymers via ATRP of PEGMA from poly(VDF-co-HFP) copolymers. No information on the cleavage of $\mathrm{C}-\mathrm{X}$ bond was provided. Incorporation of PEG chains as pendant group in the poly(VDF-co-HFP) copolymers enhanced water uptake and protein adsorption resistance. Furthermore, this grafting produced a welldistributed morphology of the blending components through the matrix compared to the nongrafted polymer, as evidenced by morphological analysis of the samples using optical microscopy and SEM.

6.4.5. From poly(VDF-co-TrFE) copolymers 
Kobayashi et al.[236] described the surface modification of poly(VDF-co-TrFE) copolymer films via surface-initiated ATRP of ${ }^{\mathrm{t}} \mathrm{BA}$ and St, resulting in grafting layers of ca. $10 \mathrm{~nm}$ (checked by AFM) as hydrophobic surfaces (Scheme 56). The reaction was monitored by NMR, ATR-IR and X-ray photoelectron (XPS) spectroscopies, and by water contact angle measurements. Actually, observation by ${ }^{19} \mathrm{~F}$ NMR spectroscopy enabled to note the C-F bond cleavage in $\mathrm{CF}_{2}$ group of TrFE units that favored the ATRP of such monomers. Subsequently, the PS grafts were heated with p-toluenesulfonic acid onto PSSA, leading to hydrophilic surface as confirmed by the decrease in the water contact angle from 91 to $15^{\circ}$.

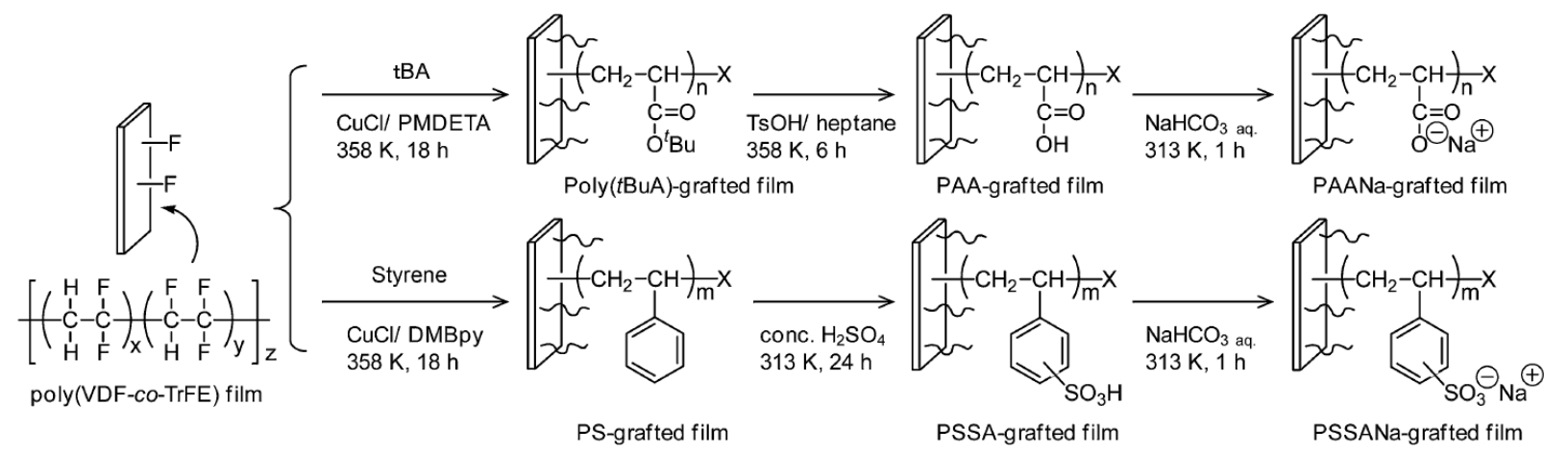

Scheme 56. Schematic diagram of the direct modification of the poly(VDF-co-TrFE) films by surface-initiated ATRP and the successive hydrolysis and sulfonation reactions.[236] Reproduced with permission from Royal Society of Chemistry.

\subsubsection{From poly(CTFE-alt-VE) copolymers}

Valade et al. [237] reported the grafting polymerization of styrene initiated by $\mathrm{C}-\mathrm{Cl}$ groups in poly(CTFE-alt-VE) and poly[(CTFE-alt-VE)-co-(HFP-alt-VE)] copolymers (where VE stands for vinyl ether) followed by the chemical modification of polystyrene grafts suitable for application in alkaline fuel cell membranes. The main advantage of using this resulting 
copolymer is that poly(CTFE-alt-VE) copolymer does not decompose in alkaline KOH solution in contrast to the VDF based copolymers. $[8,19,21]$

\subsection{Conclusion}

The above section depicts the usefulness of graft copolymers for multiple applications ranging from fuel cell membranes to functional coating materials. Apart from providing polymers with unique macromolecular architectures, graft copolymerization leads to precisely placed functionalities. Modification of commercially available fluoropolymers such as PVDF and poly(VDF-co-M) copolymers by grafting has attracted peculiar and growing interest since this depends on the chemistry, the number and molar masses of the grafts, the properties of fluoropolymers, such as surface hydrophilicity, compatibility with other polymers, adhesion to metallic and inorganic substrates which can be modified. The grafting of polymers from such a fluoropolymer backbone has been mostly achieved by conventional radical reaction via ozone treatment or high energy technologies ( $\gamma$ rays, electron beam, ionizing radiation, and plasma), followed by controlled radical polymerization techniques with the most used ATRP. In addition, $\mathrm{C}-\mathrm{Cl}$ bond in CTFE is usually very stable, but several studies led to the successful grafting of various monomers from such a bond of CTFE units in a poly(VDF-co-CTFE) copolymer. However, in all these cases, poor evidence of the grafting has been reported. Various applications of these graft copolymers are fuel cell membranes, coatings, polymer electrolytes for Li-ion battery and water treatment membranes, summarized in Section 8.

\section{Synthesis of fluorinated star/miktoarm star architecture copolymers}

Star polymers[1] offer presence of multiple functionalities in small volume, leading to fine tunability in the polymer properties. Synthesis of fluorinated star/miktoarm star architecture 
copolymers involves first polymerization via different RDRP techniques, followed by chain-end post polymerization reactions.

\subsection{Synthesis of novel PVDF-based 4-miktoarm star terpolymers}

Patil et al.[238] reported well-defined amphiphilic miktoarm polymers incorporating PVDF, PSt and PEO blocks via a combination of ATRP, ITP and CuAAC (Scheme 57). First, bis(azido)bis(dimethyl bromoester) intermediate $\underline{2}$ controlled the ATRP of styrene leading to 104 styrene units. These telechelic bis(bromo) polystyrenes ( $\underline{\mathrm{P} 3})$ were chemically changed into telechelic diiodide PVDF- $b$-PS copolymers $(\underline{\mathrm{P} 5})$ via a Finkelstein reaction, able to further allow the ITP of VDF. Finally, both azido groups in P5 BCPs reacted with $\omega$-propargyl poly(ethylene oxide) to offer miktoarm copolymer P6 containing these above copolymer sequences.

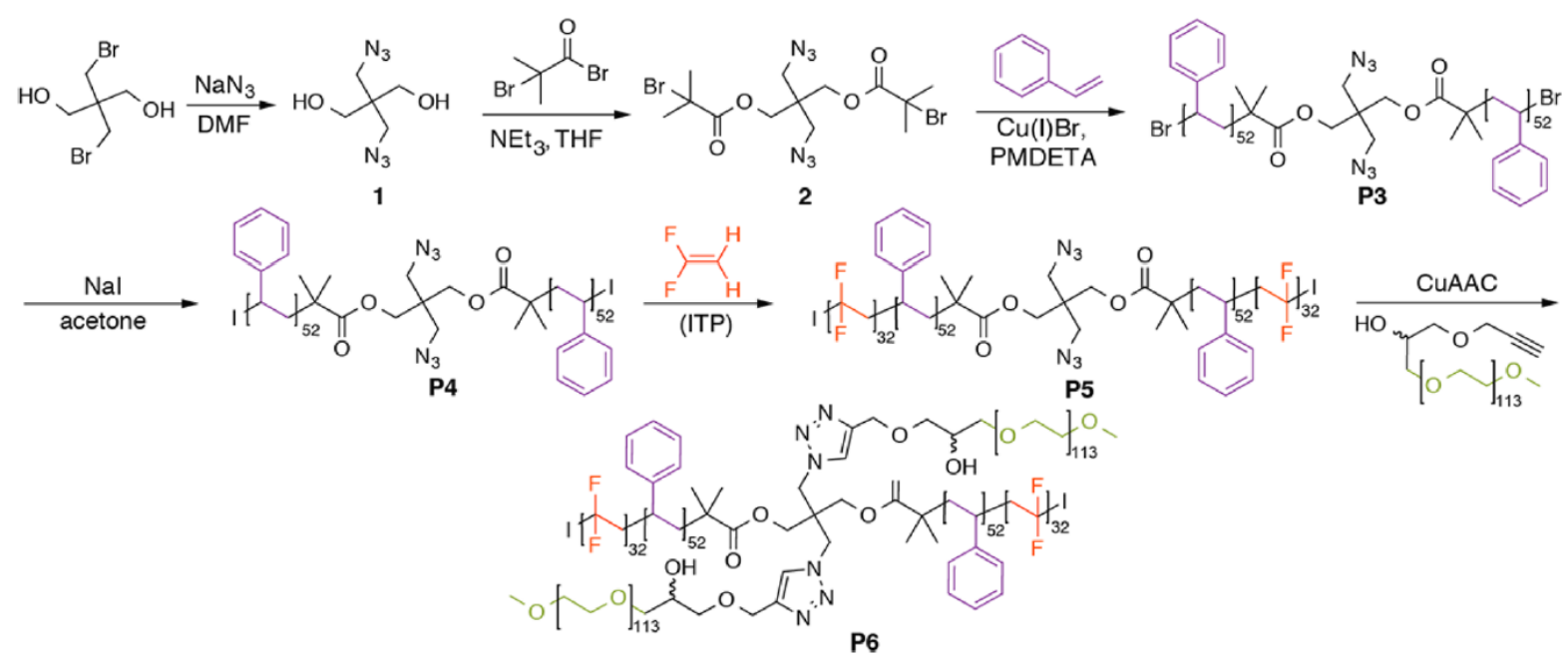

Scheme 57. Synthesis of (PVDF- $b$-PS $)_{2}(\mathrm{PEG})_{2}$ miktoarm star polymer.[238] Reproduced with permission from American Chemical Society.

\subsection{Synthesis of multi-functional PVDF Graft Copolymers}

The success of RAFT polymerization of VDF with xanthate[84, 239] was applied to a similar RAFT polymerization involving a tetra-arm xanthate CTA (Scheme 58, obtained from 
pentaerythritol) to yield a 4-arm star PVDF. Thus synthesized polymer is an appealing alternative to PVDF/ PMMA blends because it provides crosslinked PVDF transparent coatings via photocrosslinking. The advantages of this process is (a) it is very fast and versatile; (b) the coating once developed exhibited very good adhesion properties to a metal surface; and (c) both the surface energy $\left(\gamma_{c}=38 \mathrm{mN} \mathrm{m}^{-1}\right)$ and the water contact angle of the polymer films were easily tunable.

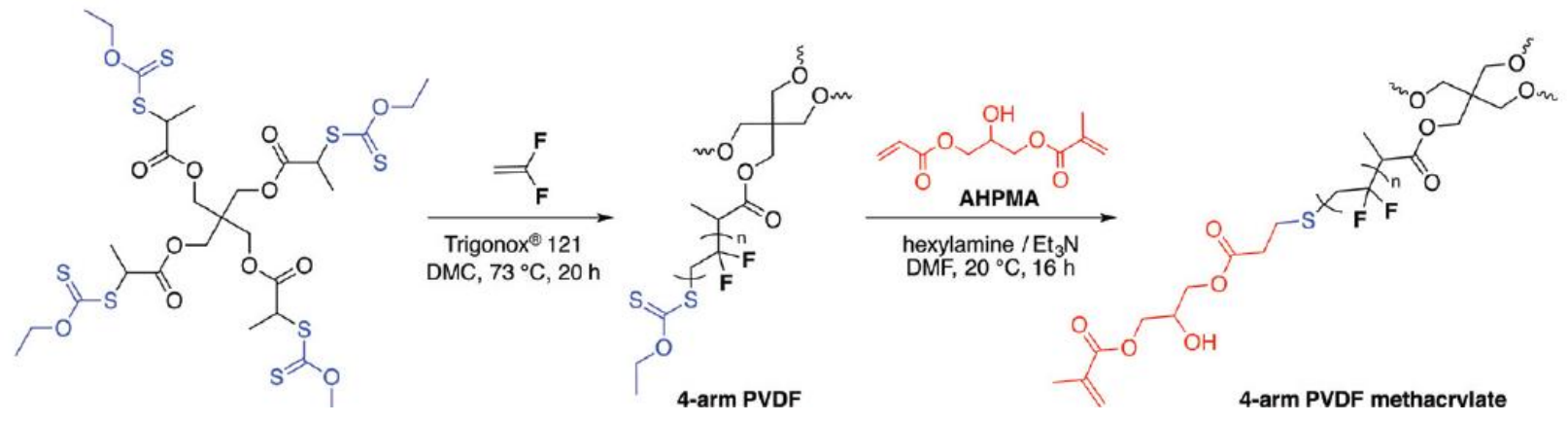

Scheme 58. Synthesis of 4-arm star PVDF methacrylate via RAFT polymerization starting from a 4-arm star initiator.[239] Reproduced with permission from Royal Society of Chemistry.

This interesting strategy was also implemented to produce star PVDF functionalized into benzaldehyde to react into tetra(triazine) PEO forming conetworks as electrolytes for Li-ion battery with quite satisfactory conductivities $\left(2-4 \mathrm{mS} \mathrm{cm}^{-1}\right) \cdot[240]$

\subsection{Conclusion}

To the best of our knowledge, quite a few stars or miktoarm copolymers containing fluorinated sequences have been reported, only from ITP or RAFT polymerizations, for polymer electrolytes for $\mathrm{Li}$ ion batteries, fuel cell membranes, antifungal and antimicrobial coatings and water treatments. To the best of our knowledge, quite a few synthesis strategies of star and miktoarm star copolymers containing fluorinated sequences have been prepared from ITP and RAFT of 
VDF.

\section{Applications}

Thanks to their remarkable properties, fluorinated polymers have been used in many high-tech applications.[2-8] This section aims at showing that such above well-defined fluorinated copolymers can be involved in specific applications, among thermoplastic elastomers, TPEs, for automotive, aerospace, aeronautics industries (e.g. elastomeric sealants, O-rings, gaskets, which are resistant to low and high temperature and in aggressive media), energy (fuel cell membranes, lithium ion batteries, actuators, and piezoelectrical devices), microfiltration and ultra-filtration membranes, blends, nanocomposites, shape memory polymer, surfactants and biomedical applications that should meet the requirement of non-toxicity, non-persistency and nonbioaccumulation.

\subsection{Thermoplastic elastomers}

A thermoplastic elastomer (TPE) contains both elastomeric (or amorphous) domains, exhibiting a low glass transition temperature and thermoplastic or crystalline zones. Beside the commercially available TPEs,[7, 8, 20, 31, 33, 38, 39, 64-66, 241] a series of synthetic fluorinated TPEs has already attracted interests of academic and industrial researchers (Scheme 23). Most often, these synthesized fluorinated copolymers-based TPEs are composed of perfluoropolyethers or from fluoroalkene-based telechelic oligomers. These fluoropolymer-based TPEs have found wide applications as O-rings and gaskets for car industry and aerospace, biomedical items and even in high resistance coatings.[242]

8.2. Fuel cells membranes

8.2.1. From poly(VDF-co-HFP)- $b$-PSSA diblock copolymers 
Tsang et al.[228] prepared poly(VDF-co-CTFE)- $g$-PSSA graft and poly(VDF-co-HFP)- $b$-PSSA diblock copolymers and compared these membrane performances processed from both copolymers. TEM analysis displayed an interconnected network of small clusters of 2-3 nm diameters, formed by the graft membranes (Figure 28).
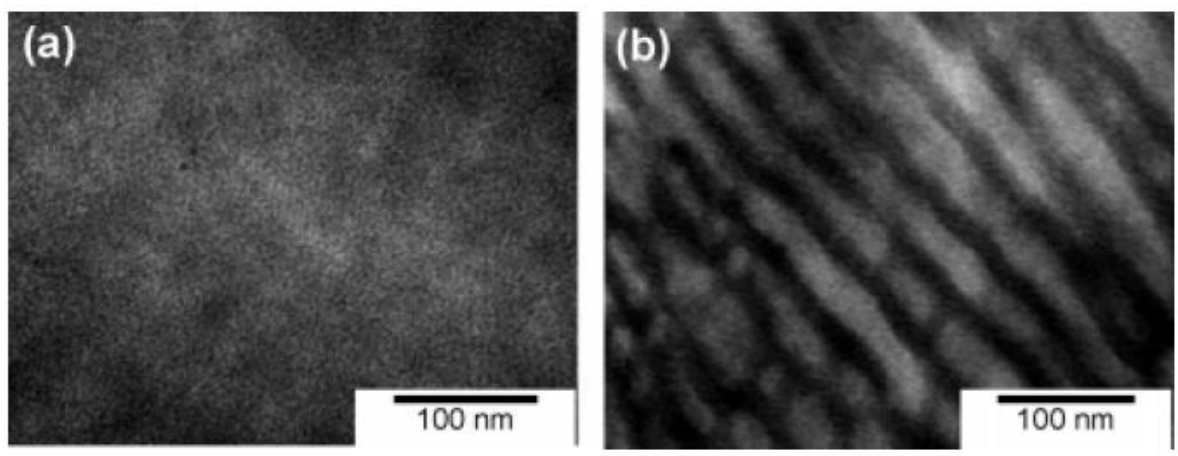

Figure 28. TEM micrographs of (a) poly(VDF-co-CTFE)- $g$-SPS (IEC $=1.95 \mathrm{mmol} / \mathrm{g}$ ) and (b) poly(VDF-co-HFP)- $b$-SPS (IEC $=0.72 \mathrm{mmol} / \mathrm{g}$ ) membranes. Note: dark areas, ionic domains; bright areas for fluorous domains.[228] Reproduced with permission from American Chemical Society.

The poly(VDF-co-CTFE)-g-PSSA graft copolymer seems better suited for such an application regarding water uptake and conductivity vs. ionic exchange capacity (IEC) and effective proton mobility (Figure 29). 

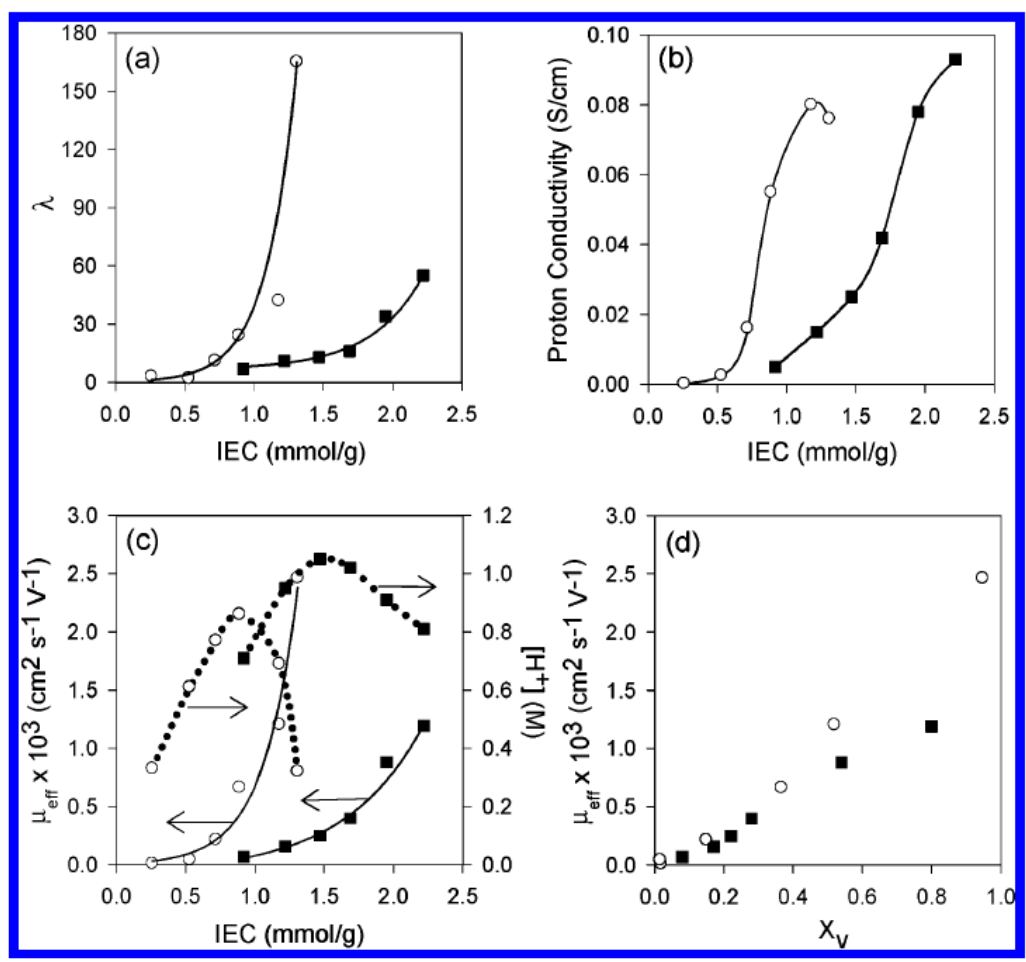

Figure 29. Plots of (a) water content (ì) vs IEC, (b) in-plane proton conductivity vs IEC, (c) effective proton mobility $\left(\mu_{\text {eff }}\right)$ (solid line) and analytical $\left[\mathrm{H}^{+}\right]$(dotted line) vs IEC, (d) $\mu_{\text {eff }}$ vs volume fraction of water (Xv) of poly(VDF-co-CTFE)- $g$-PSSA (full square symbols) and poly(VDF-co-HFP)- $b$-PSSA (empty circle symbols) proton conducting membranes.[228] Reproduced with permission from American Chemical Society.

\subsubsection{From graft copolymers prepared from poly(VDF-co-CTFE) copolymers}

Patel et al.[243] reported hybrid organic/inorganic composite polymer electrolyte membranes composed of a poly(VDF-co-CTFE)-g-PSSA graft copolymer/zeolite blend and studied for fuel cell application by varying the zeolite concentrations. A graft copolymer containing $47 \mathrm{wt} \%$ of PSSA was synthesized by ATRP and solution blended with zeolite. The water uptake capacity of the composite membranes decreased from 234 to $125 \%$ when there is an increase of the zeolite 
5A weight percent to $10 \mathrm{wt} \%$. Nanohybrid membranes for fuel cell were also reported by Jana et al.[244] from poly(VDF-co-CTFE).

Zhang et al. [205] synthesized a series of PVDF- $g$-SPS graft copolymers and then extensively studied how the polymer microstructure (backbone molar mass, graft density, graft length, sulfonic acid concentration, ion exchange capacity, etc.) affects their morphology, water uptake, and proton conductivity under various environmental conditions (temperature and relative humidity).

Wang et al.[245] reported copper phthalocyanine (CuPc)-poly(VDF-co-TrFE) composites to improve the compatibility of both components i.e., chemically modified $\mathrm{CuPc}$ oligomer was grafted to copolymer main chain. As a result, the CuPc oligomer particle size was reduced significantly, and also more uniformly distributed in the matrix as compared with the poly(VDF$c o$-TrFE)- $b$-CuPc. Consequently, the poly(VDF-co-TrFE)-g-CuPc copolymer exhibits a high dielectric constant $(\sim 100$ at $100 \mathrm{~Hz}$ and room temperature, representing a more than 6 times increase compared with that of the matrix), low dielectric loss, and a high breakdown field, all of which are highly desirable for high dielectric constant-composites.

\subsubsection{From copolymers prepared via other strategies}

Poly(VDF-co-MAF-TBE) copolymers, prepared via RAFT polymerization by Patil et al.[122] are useful emulsifiers for commercially available fluoropolymer, poly(VDF-co-HFP), and 3mercaptopropyltrimethoxysilane generating nanosilicates under reactive extrusion. The process was scaled up from a lab scale bi-screw to extruder generating rods which were calendared and produced several tenth of meters of membranes.[167] Then, the mercapto groups in these membranes were oxidized to sulfonic acid functions.[167] The resulting original membranes exhibited a theoretical ion exchange capacity of ca. 2 meq/g (whereas the experimental ones 
ranged between 1.0 and $1.3 \mathrm{meq} / \mathrm{g}$ ) and their proton conductivities reached $30-80 \mathrm{mS}^{-\mathrm{cm}^{-1} \text { at }}$ room temperature $(\mathrm{RT})$ and $100 \%$ relative humidity $\left(\mathrm{R}_{\mathrm{H}}\right)$. Interestingly, their water swelling rates were unexpectedly lower than that of the commercially available Nafion® 212.[167]

\subsection{Electroactive polymers: piezoelectric/ferroelectric/dielectric devices and actuators}

The structural versatility of block copolymers on the nanometer scale makes them suitable and highly promising candidates for many high-tech applications in soft matter nanotechnology, including optics, electronics, and acoustics. This field has recently been reviewed.[246] ITP, RAFT or conventional radical copolymerizations of fluorinated monomers and particularly of TrFE and additional monomers controlled by a xanthate or $\omega$-iodofluoroalkane CTAs[124] led thermoplastic block copolymers by virtue of this process.

ITP of VDF led to low molar mass PVDF-I $\left(\mathrm{DP}_{23}\right)$ which were vacuum vaporized into thin film that display permanent polarization of $90 \mu \mathrm{c} / \mathrm{m}$.[247] The results revealed that $\mathrm{C}_{6} \mathrm{~F}_{13}(\mathrm{VDF})_{23} \mathrm{I}$ and $\mathrm{C}_{4} \mathrm{~F}_{9}(\mathrm{VDF})_{21} \mathrm{I}$ telomer films exhibited piezoelectric responses $\left(\mathrm{d}_{33}=268 \mathrm{pC} \mathrm{N} \mathrm{N}^{-1}\right)$ and high remanent polarizations $\left(\operatorname{Pr}=98.2\right.$ and $93.4 \mathrm{mC} \mathrm{m}^{-2}$, respectively). In addition, $\mathrm{C}_{4} \mathrm{~F}_{9}(\mathrm{VDF})_{21} \mathrm{C}_{2} \mathrm{H}_{4} \mathrm{I}$ exhibited interesting ferroelectric properties $\left(\mathrm{Pr}=89.9 \mathrm{mC} \mathrm{\textrm {m } ^ { - 2 }}\right)$. More applied studies from low PVDF-I molar masses were reported by several groups. First, Noda et al.[248] studied the formation and visualization of local polarized domains in VDF oligomer thin films by using AFM. The formed domain size depended on the pulse poling conditions. Also, the domain size increased with the magnitude and the duration of a pulse voltage are increased. A local domain with a diameter of $65 \mathrm{~nm}$ was successfully created. Meijer's group[249] proposed an end-functionalization method of PVDF oligomers (I-OVDF) via a radical reaction between terminal olefins and I-OVDF (Scheme 59). This study demonstrates 
the potential of preparing a new set of ferroelectric materials simply by attaching OVDF oligomers onto different materials. Thus developed material has potential use to prepare devices with ferroelectricity and electrical conductivity or light-harvesting properties.

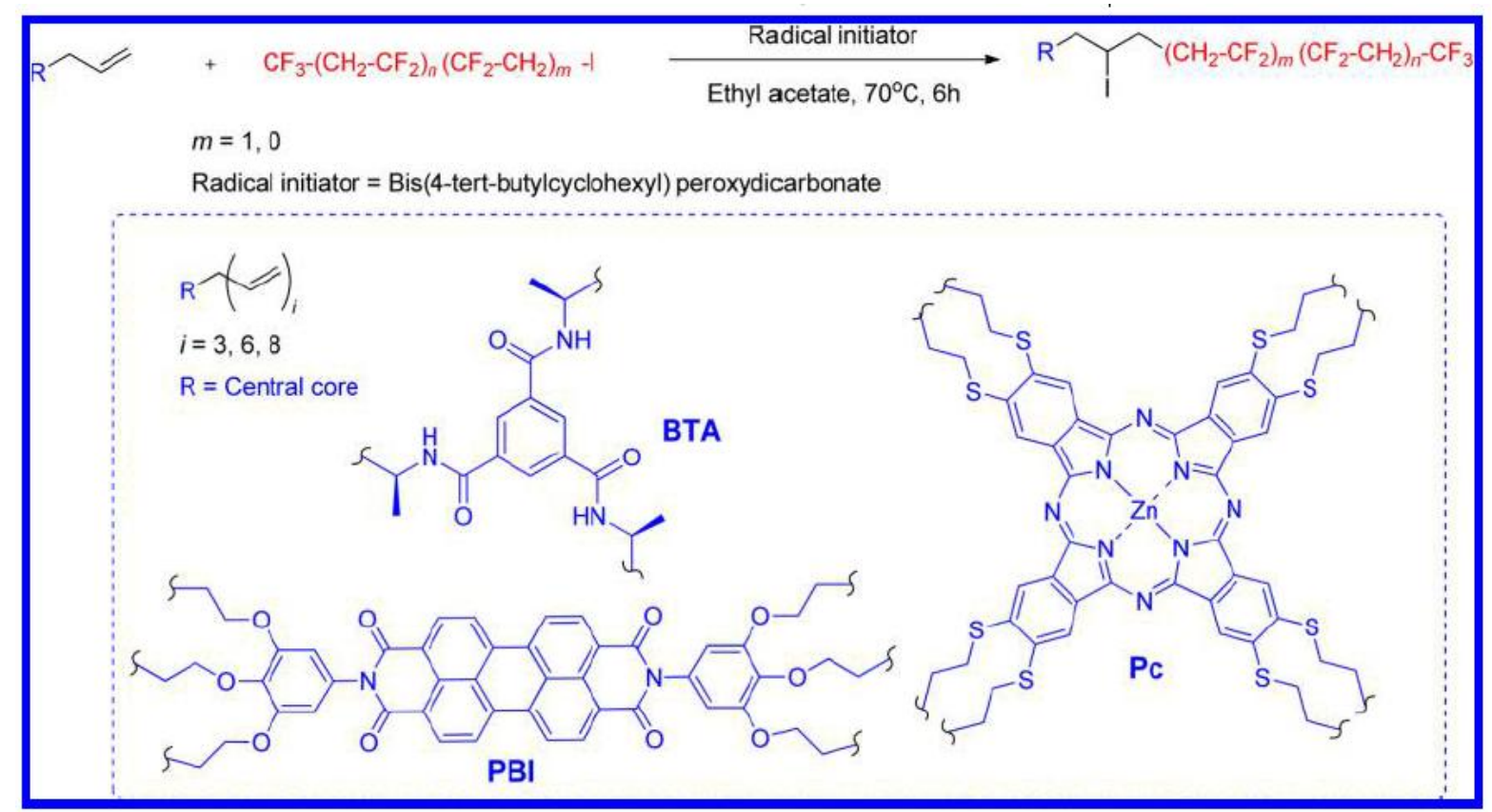

Scheme 59. Synthetic method for the end-functionalization of oligo(VDF).[249] Reproduced with permission from American Chemical Society.

Wang et al.[223] developed a polymer dielectric via grafting PMMA onto main chains of poly(VDF-ter-TrFE-ter-CTFE) terpolymer via ATRP. This material has potential application in producing composite dielectrics bearing simultaneously high energy density and high discharging efficiency.

Terzic et al.[183] reported that the incorporation of P2VP chains at both ends of poly(VDF-coTrFE) central block in P2VP- $b$-poly(VDF-co-TrFE)- $b$-P2VP triblock copolymer and their phase separation do not induce drastic changes in the shape of the D-E loops. An exchange of P2VP with PS of the same molar mass generates considerably different switching characteristics. D-E 
loops become narrower, resembling linear dielectric behavior, with almost zero remanent polarization and the maximum polarization lower than for P2VP-b-poly(VDF-co-TrFE) 70:30. Zhu's group[217] explored nanoconfinement from poly(VDF-ter-CTFE-ter-TrFE)-g-PS related to the confinement (or insulation) of poly(VDF-co-TrFE) ferroelectric (FE) crystals. In this model, after crystallization, PS side chains were segregated to the periphery of poly(VDF-coTrFE) crystals leading to a nanoscale PS interfacial layer. Because of the low polarizability of this confining layer, the compensation polarization at the amorphous-crystalline interface decreased while the local polarization field became weaker than that of pure poly(VDF-co-TrFE) copolymer. The PS side chains, covalently bonded to the poly(VDF-co-TrFE) polymer backbone behaved as an anchor in the amorphous phase thus preventing dipoles flipping along the electric field.

The incorporation of PEMA side chains into poly(VDF-ter-TrFE-ter-CTFE) changes the Displacement-Electric field (D-E) hysteresis loops from a relaxor ferroelectric (RFE) behavior to that of anti-ferroelectric. Hence, it appears that amorphous PEMA could confine the crystal. Similarly, to PS grafted copolymers, the anti-ferroelectric tendency of those graft copolymers was preserved after repeated poling cycles but is closely related to the PEMA amount. Regarding the discharge energy density (D), a non-compatible side chain such as PS or a miscible one (PEMA) did not clearly gave clearly different results (Figure 30).[225] The origin of the antiferroelectric behavior stems from the introduction of gauche-type linkage induced by the grafted chains. These linkages are quite stable under high electric field and cannot be modified into trans conformations. Compared to e-beam irradiated poly(VDF-co-TrFE) and poly(VDF-ter-TrFE-terCFE) terpolymer, higher discharged energy densities were noted (Figure 30). This may arise from the lower $D_{\max }$ values of the graft copolymer compared to those of the RFE polymers which 
contains lower amount of all-trans conformations of poly(VDF-co-TrFE) chains. However, the

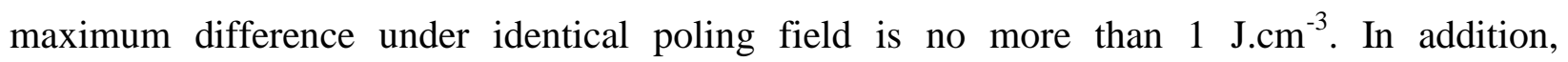
poly(VDF-ter-TrFE-ter-CFE) and e-beamed poly(VDF-co-TrFE) copolymers exhibit broadened hysteresis loops at $75{ }^{\circ} \mathrm{C}$. The higher Curie temperature for the poly(VDF-ter-TrFE-ter-CTFE)$g$-PS (14\%) grafted copolymer may be advantageous for applications above $75{ }^{\circ} \mathrm{C}$.

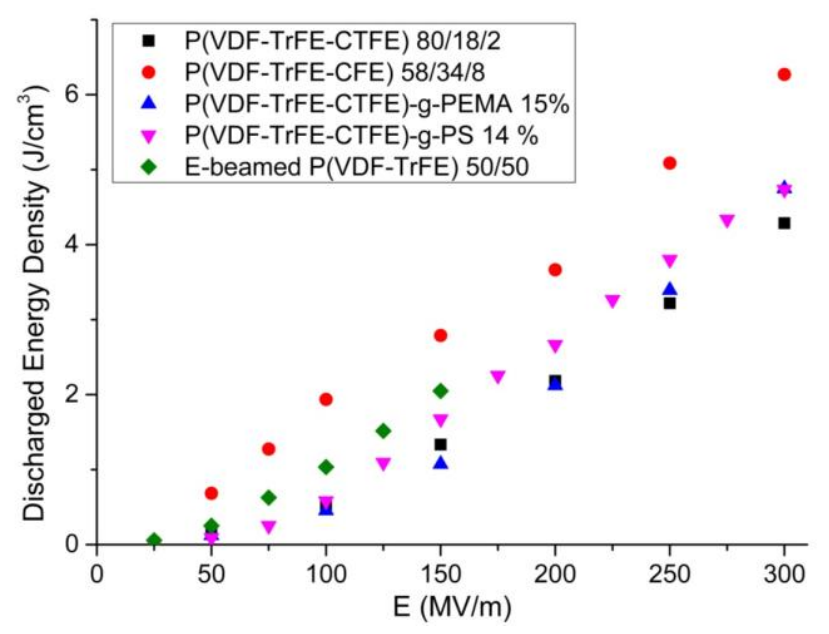

Figure 30. Comparison of discharged energy densities (D) for 80/18/2 mol \% poly(VDF-terTrFE-ter-CTFE) from ref,[225] 58/34/8 mol \% poly(VDF-ter-TrFE-ter-CFE) from ref,[250] poly(VDF-ter-TrFE-ter-CTFE)-g-PEMA (15 mol \%) from ref,[225] poly(VDF-ter-TrFE-terCTFE)- $g$-PS (14 mol \%) from ref[251] and e-beam irradiated 50/50 mol \% poly(VDF-co-TrFE) from ref.[252] Reproduced with permission from Elsevier.

In addition, Meereboer et al.[253] synthesized a series of PVDF-based triblock copolymers and demonstrated how crystallization is affected by the morphology and chemical nature of the amorphous block. These results may provide a guideline for the process to obtain crystalline $\beta$ phases of PVDF suitable for electroactive applications in advanced fields. 


\subsection{High energy storage capacitors}

A separator membrane separates the anode and cathode in lithium-ion batteries and also controls the number and mobility of the lithium ions. Among the polymer matrices most commonly investigated for battery separators are PVDF[254] and VDF copolymers such as poly(VDF-coTrFE), poly(VDF-co-HFP) and poly(VDF-co-CTFE), due to their excellent properties such as high polarity and the possibility of controlling the porosity of the materials through binary and ternary polymer/solvent systems.[255] Costa et al.[256] reviewed the battery separators based on VDF homo- and co-polymers for lithium ion batteries and mentioned a wide range of fluoropolymers involved for composites and polymer blends. For each family, that review reports the main features for battery separator membrane and $\mathrm{Li}^{+}$ion transport. For the same application, Xiao et al.[257] reported macroporous polymer electrolytes based on poly(PEO)- $b$ PMMA block copolymers blends, prepared by a phase inversion technique.

\subsection{Surfactants}

Most fluorinated surfactants, including perfluorooctanoic acid (PFOA, $\mathrm{C}_{7} \mathrm{~F}_{15} \mathrm{CO}_{2} \mathrm{H}$ ) and perfluorooctanesulfonic acid (PFOS, $\mathrm{C}_{8} \mathrm{~F}_{17} \mathrm{SO}_{3} \mathrm{H}$ ), are much more efficient than their hydrocarbon counterparts.[258] But, they are toxic, bioaccumulable and persistent. The perfluorinated moieties in PFOA and PFOS are too stable to be degraded metabolically or by other means. Research on finding better alternatives to such surfactants started in 2006 via the Stewardship Program.[156] However, it is still a great challenge to find such derivatives by use of hydrogenofluorinated surfactants and copolymers/cotelomers based on VDF are of growing interest. Kostov et al.[83] synthesized poly(VDF-co-3,3,3-trifluoropropene)- $b$-oligo(vinyl alcohol) block copolymers by sequential RAFT copolymerization of VDF and 3,3,3trifluoropropene (TFP), followed by a chain extension of VAc in the presence of xanthate and 
finally the hydrolysis of the PVAc sequence to achieve original surfactants. These amphiphilic copolymers display quasi similar surface tension as ammonium perfluorooctonoate (APFO) and with an advantageous possible cleavable/degradable poly(VDF-co-TFP) in contrast to perfluorinated moieties in APFO. On the other hand, fluorinated block copolymers are interesting since the fluorous sequence is $\mathrm{CO}_{2}$-philic and can be used in $\mathrm{scCO}_{2}$. One example was suggested by Girard et al.[157] who synthesized original VDF-based amphiphilic block copolymers by RAFT/MADIX polymerization containing poly(VDF-co-PMVE) sequence, soluble in $\mathrm{scCO}_{2 \cdot[158]}$

\subsection{VDF-based amphiphilic diblock copolymers}

Park et al.[259] prepared well-defined amphiphilic PMMA- $b$-PEGMA block copolymers via ATRP (Scheme 60). This copolymer was used as additives in DMAc casting solution to fabricate PVDF ultra-filtration (UF) membranes.

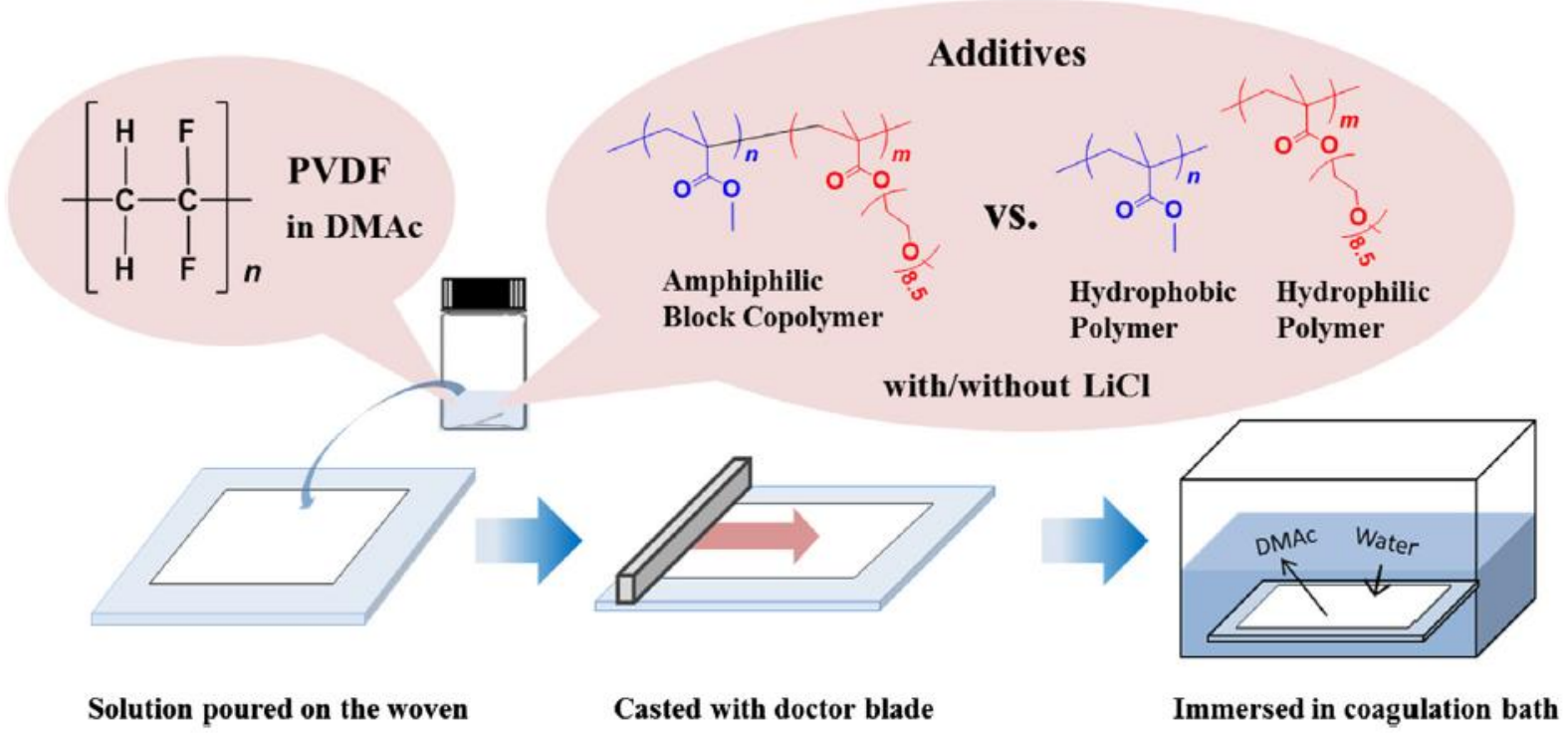

Scheme 60. Schematic representation for the fabrication of porous membranes from amphiphilic

PMMA- $b$-PEGMA/PVDF block copolymer blends by NIPS process.[259] Reproduced with permission from Elsevier. 


\subsection{Self-assembly}

Self-assembly of block copolymers has the potential to develop smart materials for advanced applications and more recent studies, involving fluorinated block copolymers, have been developed and summarized below.[260, 261]

\subsubsection{Polymerization-Induced Self-Assembly (PISA) of VDF into PVAc- $b$-PVDF block copolymers}

Quite a few articles have been reported in the literature on PISA of fluorinated alkenes. Many recent articles and reviews have shown that due to easiness to prepare block copolymers, especially amphiphilic ones, they have been extensively used to produce nano objects that selfassemble in various media during the polymerization (PISA).[260] Guerre et al.[155] synthesized and self-assembled in water an amphiphilic PVDF- $b$-PVA block copolymer, produced via sequential RAFT copolymerization of VDF and VAc, followed by the hydrolysis of PVAc block into PVA (Scheme 28). The self-assembled nanoparticles (of ca. $100 \mathrm{~nm}$ ) were characterized by both DLS and cryo-TEM (Figure 31).

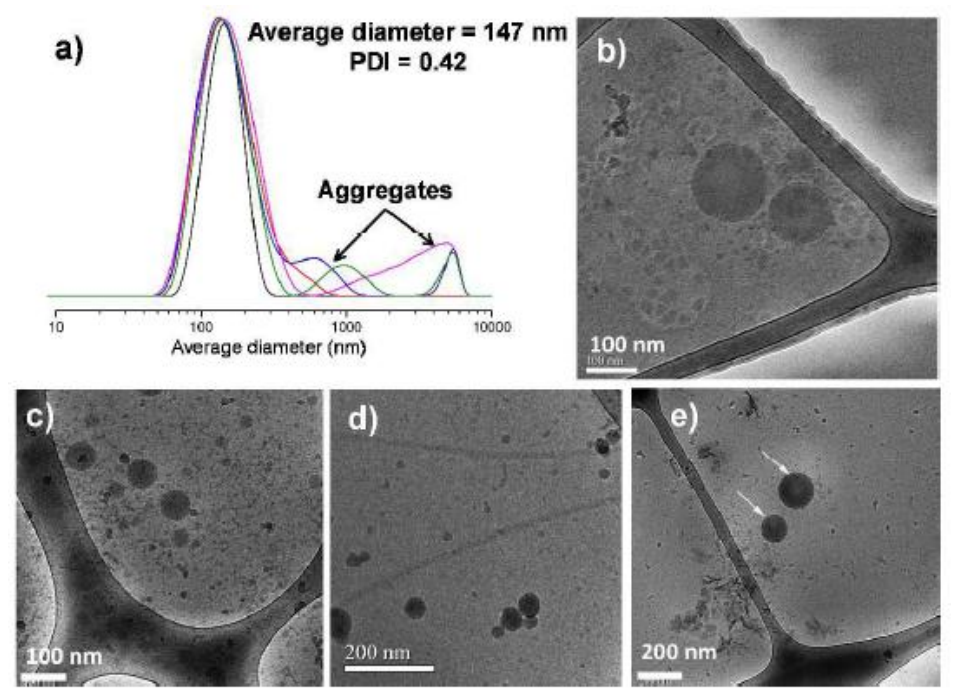

Figure 31. a) Intensity-average hydrodynamic diameter distribution of $\mathrm{PVDF}_{51}-b-\mathrm{PVA}_{172}$ 
amphiphilic block copolymers self-assembled in water $(\mathrm{c}=1.16 \mathrm{mg} / \mathrm{mL})$ measured by DLS. b, $\mathrm{c}$, $\mathrm{d}$, e) Cryo-TEM micrographs of the $\mathrm{PVDF}_{51}-b-\mathrm{PVA}_{172}$ nanoobjects self-assembled in water.[155] Reproduced with permission from Royal Society of Chemistry.

The same team[262] also performed PISA of VDF from PVAc-Xa to obtain PVAc- $b$-PVDF block copolymers in DMC under RAFT dispersion polymerization condition (Scheme 61). The authors suggested that the structures were formed by epitaxial growth of PVDF crystals and interparticle cross-penetration crystallization while the morphologies of these BCP structures were governed by the crystallization of PVDF.

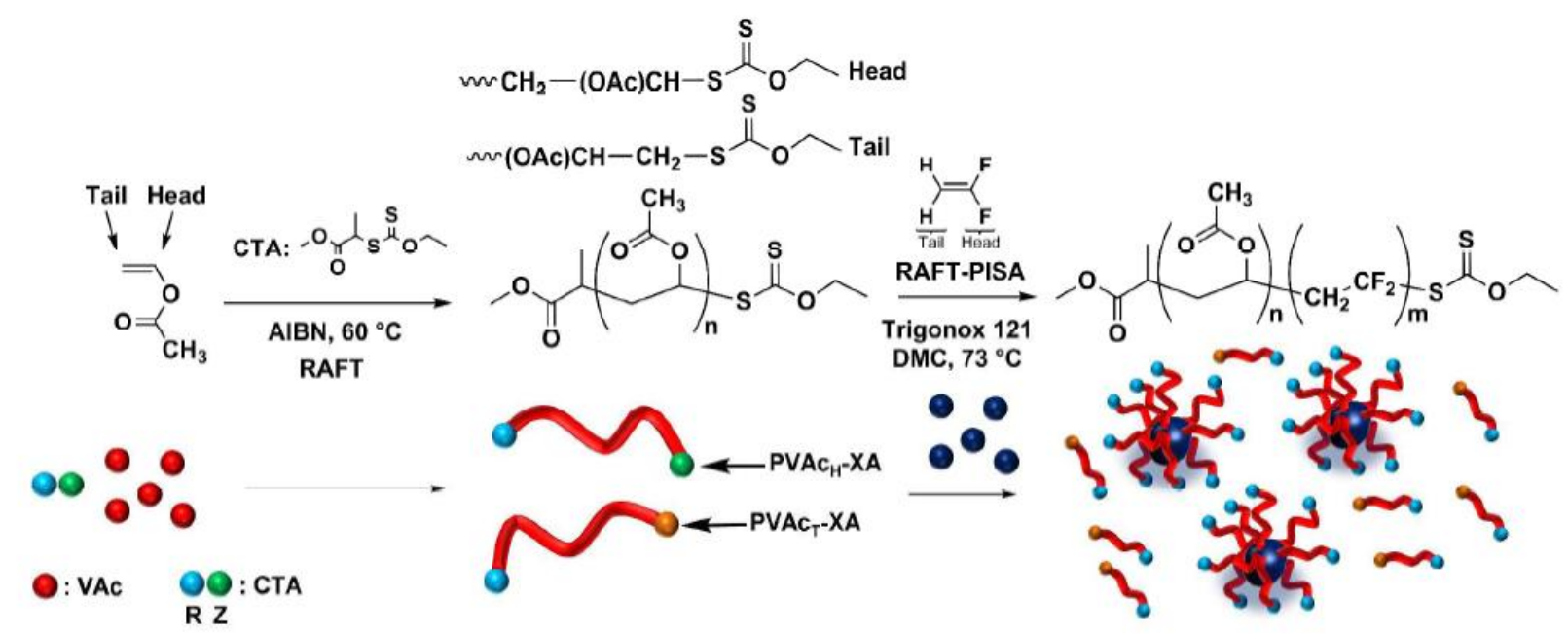

Scheme 61. Synthesis and self-assembly of PVAc- $b$-PVDF block copolymers by RAFT dispersion polymerization of VDF in DMC using PVAc macro-CTA.[262] Reproduced with permission from Royal Society of Chemistry.

In another strategy, Guerre et al.[182] prepared amphiphilic PVDF- $b$-PDMAEMA block copolymers which undergoes crystallization-driven self-assembly in water at $\mathrm{pH} 2,8$ (native $\mathrm{pH}$ ) and 10. Morphological analysis of the self-assembled structures revealed mainly polydisperse (20-500 nm) and roughly spherical aggregates (Figure 16). 


\subsubsection{Self-assembly of PVDF- $b$-PS block copolymers}

Wu et al.[263] reported preliminary solution self-assembly behaviors of PVDF- $b$-PS block copolymers. The self-assembly experiments were performed in a series of binary solvent mixtures containing a good solvent (DMF) and a selective solvent with different ratios. The results revealed that the self-assembly process of the block copolymer was affected by (a) the length of the PS block and (b) the solvent composition (Figure 32).

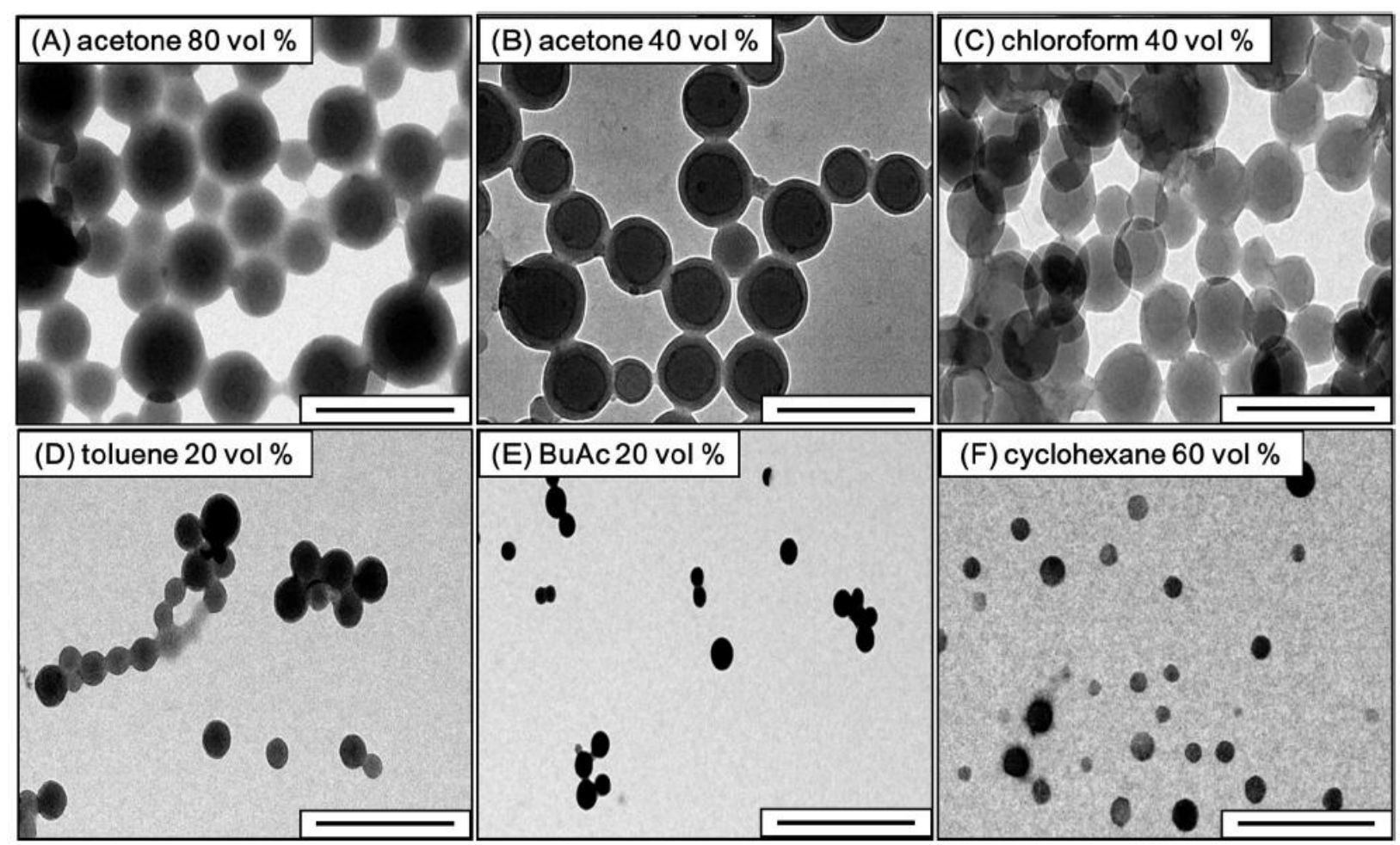

Figure 32. Representative TEM images of micelles formed by $\mathrm{PVDF}_{180}-\mathrm{PS}_{1202}$ in different solvent conditions. (A) and (B) DMF/acetone, (C) DMF/chloroform, (D) DMF/toluene, (E) DMF/BuAc, (F) DMF/cyclohexane. Scale bars: 500 nm.[263] Reproduced with permission from Elsevier.

\subsubsection{Self-assembly of PVDF- $b$-PEG- $b$-PVDF triblock copolymers}


The self-assembly of the PVDF- $b$-PEG- $b$-PVDF triblock copolymers, prepared by Folgado et al.[159] was studied in water and ethanol. Both the induced micellization (via dropwise addition of a selective solvent for PVDF to a solution of the triblock) and nanoprecipitation (dropwise addition of a solution of the triblock into a non solvent of PVDF) led to formation of micelles (Figure 33) and vesicles (Figure 34). Surprisingly, under nanoprecipitation conditions (in THF/ ethanol), well-defined crystalline micrometric structures were obtained.

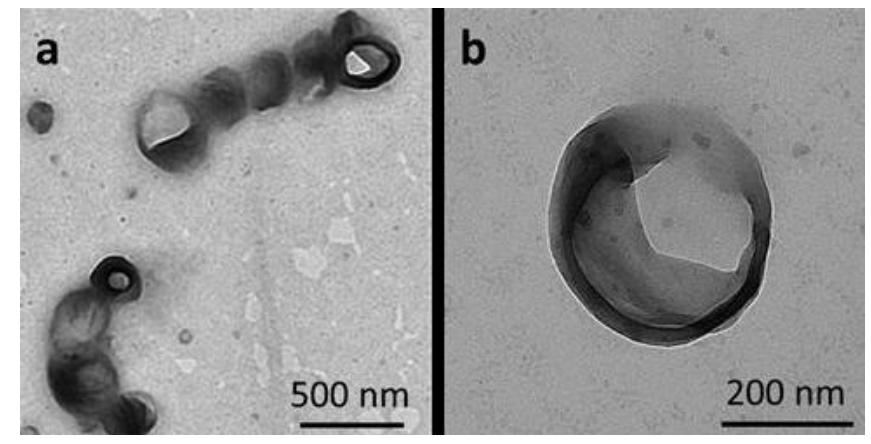

Figure 33. TEM images of $\mathrm{PVDF}_{50}-b-\mathrm{PEG}_{136}-b-\mathrm{PVDF}_{50}$ aggregates obtained from: a) and $\mathrm{b}$ ) $1 \% \mathrm{w} / \mathrm{w}$ solution in THF by micellization (THF: ethanol (1:6).[159] Reproduced with permission from Royal Society of Chemistry.

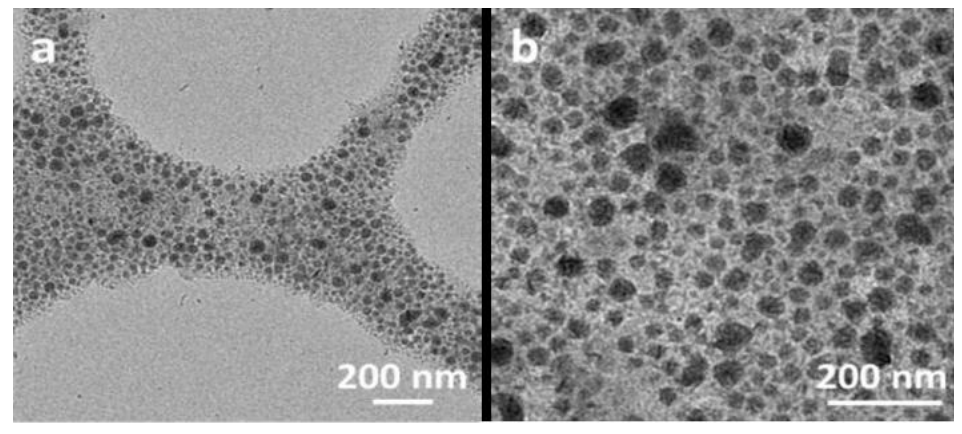

Figure 34. TEM images of $\mathrm{PVDF}_{50}-b-\mathrm{PEG}_{136}-b-\mathrm{PVDF}_{50}$ aggregates obtained $\mathrm{f}$ aggregates obtained from a 5\% w/w solution in NMP by nanoprecipitation (NMP:water (1:6)).[159] Reproduced with permission from Royal Society of Chemistry.

\subsection{Composites}


Extensive research and development have been carried out on organic/inorganic composite materials.[30, 264-266] Typically, when inorganic phases in organic/inorganic composites have nanometer dimensions, they are called nanocomposites. Organic/inorganic nanocomposites are generally composed of organic polymer composites with inorganic nanoscale building blocks.

8.8.1. Nanocomposites Based on PMMA/PVDF Blends and Multiwalled Carbon Nanotubes Lee et al.[267] prepared PVDF/PMMA blend nanocomposites with multiwalled carbon nanotubes (CNTs) by melt mixing and hot press molding. Addition of CNTs to the PMMA/PVDF blend promoted the crystallization and, more especially, the formation of $\beta$ crystals, as evidenced by the XRD analysis (Figure 35).

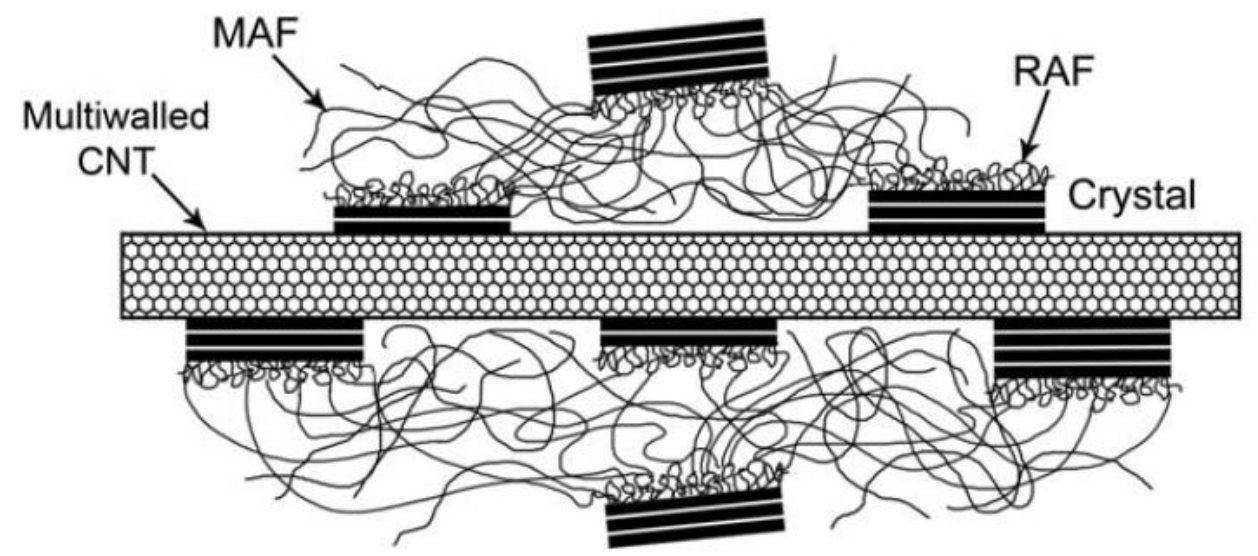

Figure 35. Schematic illustration of morphology around CNT considering three-phase model, i.e. mobile amorphous fraction (MAF), rigid amorphous fraction (RAF) and crystalline phase.[267] Reproduced with permission from Wiley.

\subsubsection{Gold-fluoropolymer nanocomposites}

Safonov et al.[268] reported development of degradation-resistant 2D Au NP-fluoropolymer composites. The composite was prepared by coating oligo(hexafluoropropylene oxide) based 
fluoropolymer thin film on Au surface by hot wire chemical vapour deposition (HWCVD) and ion sputtering. The protective coating could prevent the plasmonic properties of the Au NPs from changing for several months.

8.8.3. Polymer nanocomposites based on PVDF/PVC doped with graphene nanoparticles Elashmawi et al.[269] reported preparation of novel nanocomposites based on PVDF/PVC blend and graphene oxide (GO) nanoparticles. The addition of $\mathrm{GO}$ prompts a crystal transformation of $\alpha$-phase of PVDF, as revealed by IR analysis. The structural change of the PVDF/PVC blend before and after adding GO were studied by XRD analysis (Figure 36).

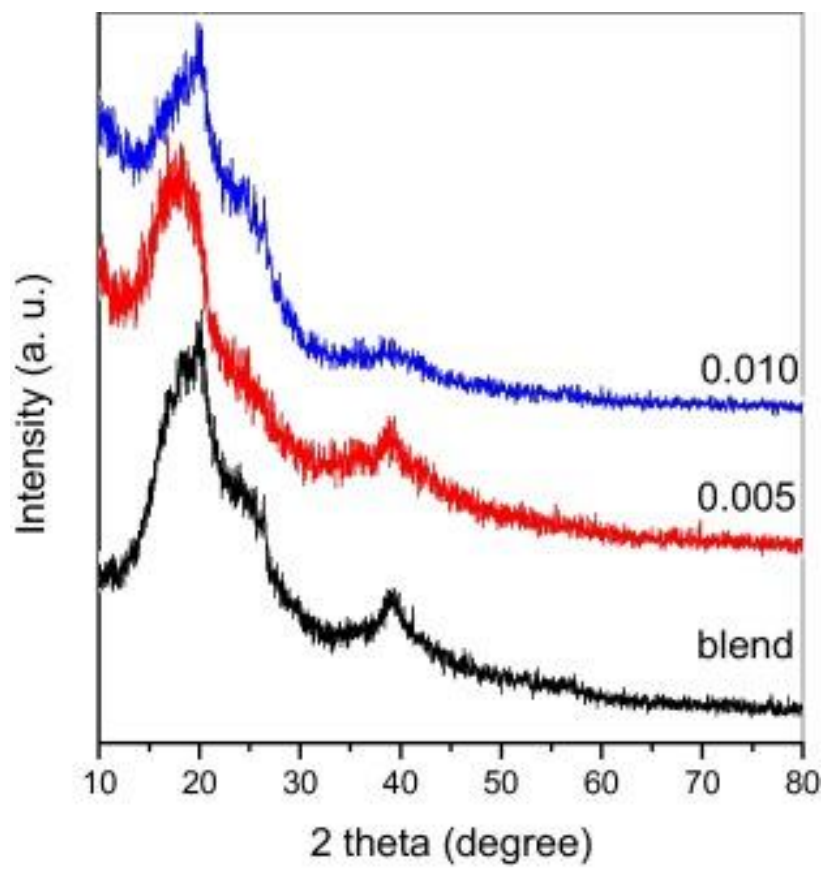

Figure 36. The X-ray diffraction scan of PVDF/PVC/Graphene nanocomposites (pure PVDF/PVC doped 0.005 and 0.010 wt.\% of graphene oxide).[269] Reproduced with permission from Elsevier.

\subsubsection{Surface functionalization of $\mathrm{BaTiO}_{3}$ nanoparticles}

In order to prepare super-capacitors, Bouharras et al.[270] achieved core-shell structured PVDF- 
grafted- $\mathrm{BaTiO}_{3}$ nanocomposites via RAFT polymerization of VDF controlled by xanthate functions decorating $\mathrm{BaTiO}_{3}$ modified-particles (Scheme 62).

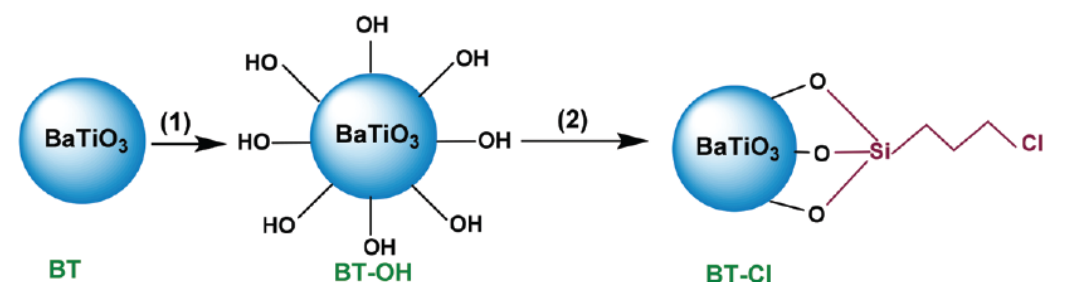

$\mathrm{BT}-\mathrm{Cl}$

(1) $\mathrm{H}_{2} \mathrm{O}_{2}, 105{ }^{\circ} \mathrm{C}$

(2) $\mathrm{ClCH}_{2} \mathrm{CH}_{2} \mathrm{CH}_{2} \mathrm{Si}\left(\mathrm{OCH}_{2} \mathrm{CH}_{3}\right)_{3}, 80{ }^{\circ} \mathrm{C}$

(3) $\mathrm{C}_{2} \mathrm{H}_{5} \mathrm{OC}(\mathrm{S}) \mathrm{SK}, 80^{\circ} \mathrm{C}$

(4) $\mathrm{H}_{2} \mathrm{C}=\mathrm{CF}_{2}, \mathrm{TBPPi}^{\circ} 65^{\circ} \mathrm{C}$

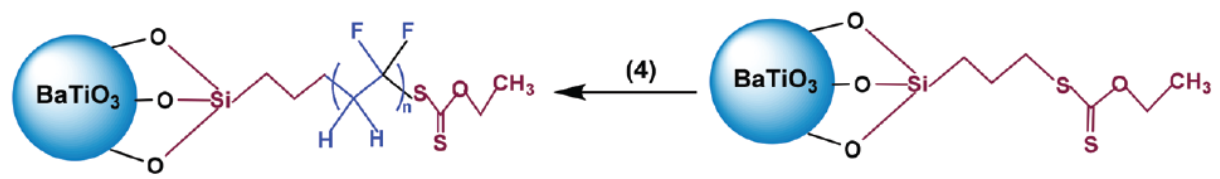

PVDF-g-BT

BT-XA

Scheme 62. Sketch illustrating the synthesis process of PVDF- $g$ - $\mathrm{BaTiO}_{3}$ nanocomposites by RAFT polymerization of VDF in the presence of xanthates (from the modification of $\mathrm{BaTiO}_{3}$ nanoparticles) where TBPPi stands for tert-butyl peroxypivalate.[270] Reproduced with permission from Royal Society of Chemistry.

The presence of PVDF linked to xanthate was evidenced by High Resolution Magic Angle Spinning (HR-MAS) NMR that highlighted the characteristic NMR signals of VDF units, including that adjacent to $\mathrm{Z}$ group (i.e. $-\mathrm{CH}_{2} \mathrm{CF}_{2} \mathrm{SC}(=\mathrm{S}) \mathrm{OEt}$ ) moieties at $-71 \mathrm{ppm}$. Transmission electron microscopy displayed the presence of thin shell of PVDF (the thickness varying from 2.2 to $5.1 \mathrm{~nm}$ ) around the $\mathrm{BaTiO}_{3}$ nanoparticles forming a core-shell structure (Figure 37). Thermal property analysis of the nanocomposite revealed that addition of a small amount of the nanoparticle as filler to the polymer matrix increased the melting temperature of PVDF from 168 
${ }^{\circ} \mathrm{C}$ to $173{ }^{\circ} \mathrm{C}$ and reduced the crystallinity of PVDF from $47 \%$ to $21 \%$. Such developed materials have potential application for high energy storage capacitors.
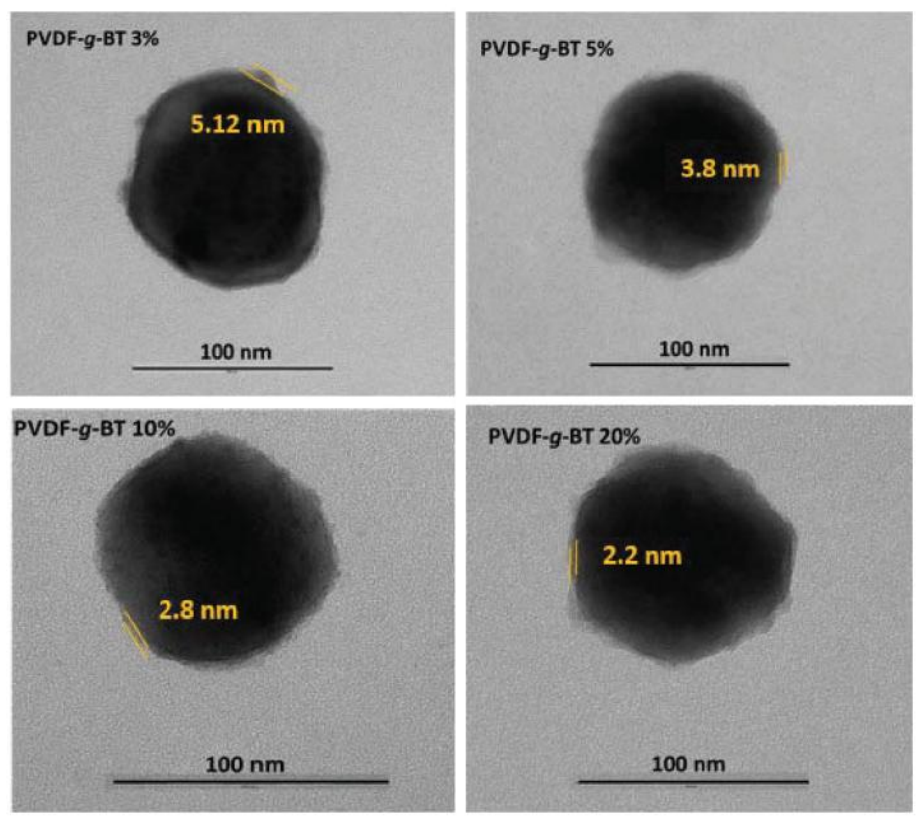

Figure 37. TEM images of $\mathrm{PVDF}-\mathrm{g}-\mathrm{BaTiO}{ }_{3}$ nanocomposites with different $\mathrm{BaTiO}_{3}$ concentrations (3, 5, 10 and 20\%).[270] Reproduced with permission from Royal Society of Chemistry.

Xie et al.[271] prepared conductive polypyrrole (PPy) nanoparticle- $\mathrm{BaTiO}_{3}(\mathrm{BT})$ composites and then incorporated it into PVDF to prepare the composites. Dielectric property measurements revealed that the PPy@BTOH composite particles endowed the PVDF-PPy@BTOH composites with extremely high $\varepsilon^{\prime}$, ultralow $\tan \delta$ and high $\mathrm{E}_{\mathrm{bd}}$ compared with the PVDF/PPy@BT composites where PPy nanoparticles and $\mathrm{BaTiO}_{3}$ particles are dispersed in PVDF dissociatively. The authors also proposed the polarization and loss mechanisms of the composites based on their morphologies and the microstructures. 


\subsubsection{Surface functionalization of $\mathrm{SiO}_{2}$ nanoparticles}

Iodine transfer terpolymerization of VDF with HFP and 2-trifluoromethacrylic acid (Scheme 63) enabled us to prepare nanocomposites based on either submicronic silica[272] or anatase titanium oxide.[273] It was observed that the nanosilica particles were well-dispersed within the fluorinated matrix (Figure 38) and that the resulting nanocomposites exhibited quite high thermostabilities, since at $800{ }^{\circ} \mathrm{C}$, they were still stable (loosing $20 \mathrm{wt} \%$ only) in contrast to the terpolymer that was totally decomposed (Figure 39).

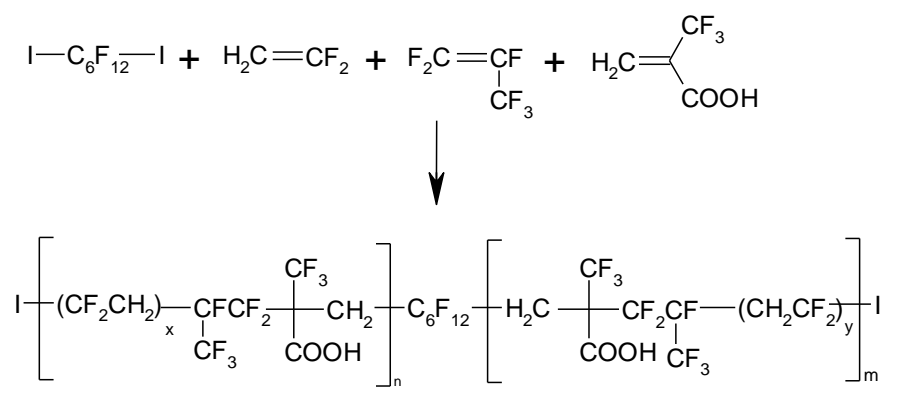

Scheme 63. Iodine Transfer terpolymerisation of VDF with HFP and 2-trifluoromethacrylic acid (MAF) in presence of 1,6-diiodoperfluorohexane.[272]

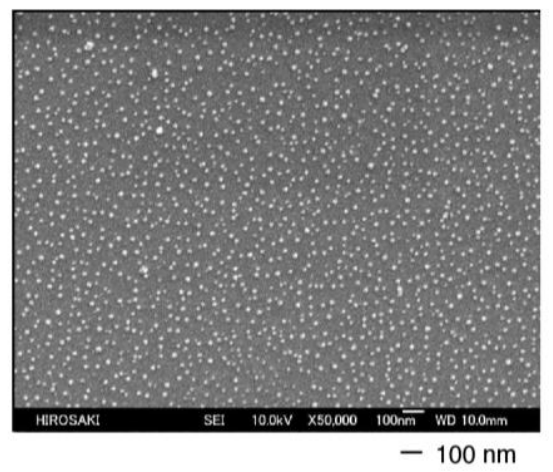

Figure 38. SEM image of a nanocomposite based on poly(VDF-ter-HFP-ter-MAF) terpolymer /silica (the scale is $100 \mathrm{~nm}$ ).[272] Reproduced with permission from American Chemical Society. 


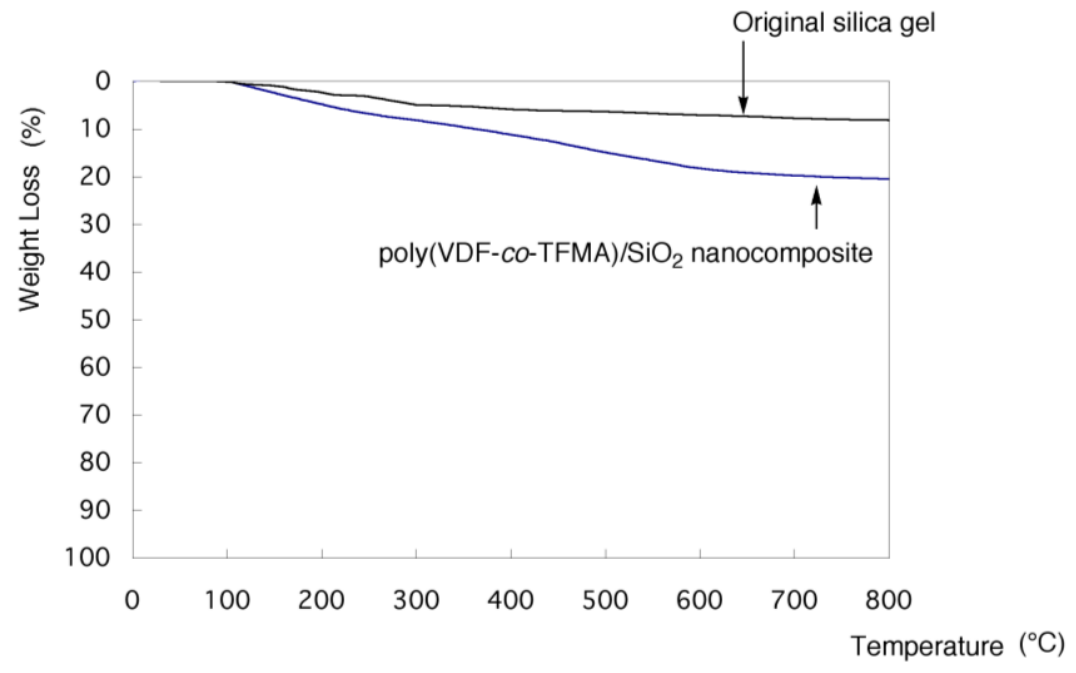

Figure 39. TGA thermograms of silica and nanocomposite based on poly(VDF-ter-HFP-terMAF) terpolymer and silica.[272] Reproduced with permission from American Chemical Society.

Furthermore, Durand et al.[274] attached PVDF-I onto silica bearing vinyl bonds on its surface (Scheme 64) for coating applications.[275]

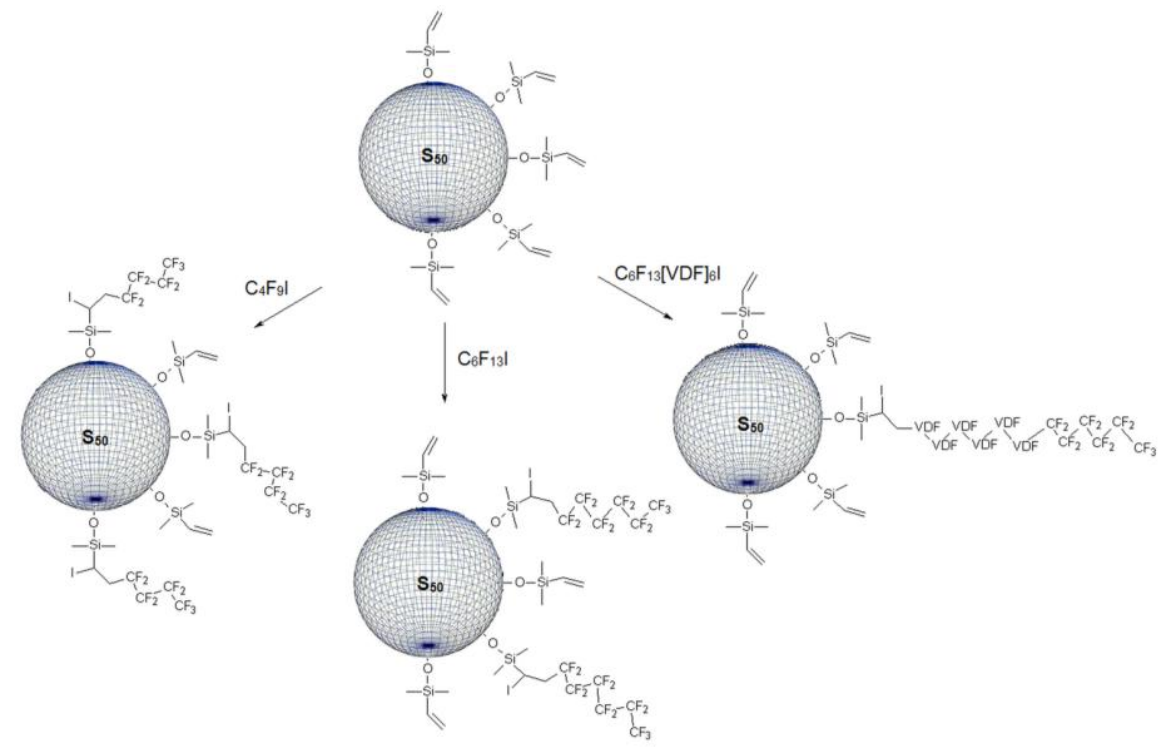

Scheme 64. Radical addition of $\mathrm{C}_{4} \mathrm{~F}_{9} \mathrm{I}, \mathrm{C}_{6} \mathrm{~F}_{13} \mathrm{I}$ and PVDF-I onto a functionalized vinyl silica[274] 
Reproduced with permission from American Chemical Society.

\subsubsection{Nanocomposites Based on PVDF/PMAA/Ni nanocomposites}

Loos' team produced original PVDF/PMAA/Ni nanocomposites via selective hydrolysis of the tBMA segments of the PtBuMA- $b$-PVDF- $b$-PtBuMA triblock copolymer (prepared by ATRP, section 5.4.1, Scheme 35) and subsequent backfilling of the remaining polymer template with nickel metal through electroless metal deposition. The $\beta$-polymorph, as well as the lamellar morphology was maintained even after the hydrolysis and electroless plating. A similar pathway was used in the presence of ATRP of styrene.[276]

\subsubsection{Nanocomposites containing cobalt ferric oxide}

The same authors also studied multiferroic nanocomposites containing both magnetic cobalt ferrite (modified by gallic acid, selectively incorporated in P2VP domains due to strong hydrogen bonds formation) and P2VP-b-poly(VDF-co-TrFE)- $b$-P2VP block copolymers (Scheme 40).[277] Different cobalt ferric oxide (CFO) loadings were used to prepare at least four nanocomposites that were characterized by SAXS (Figure 40).
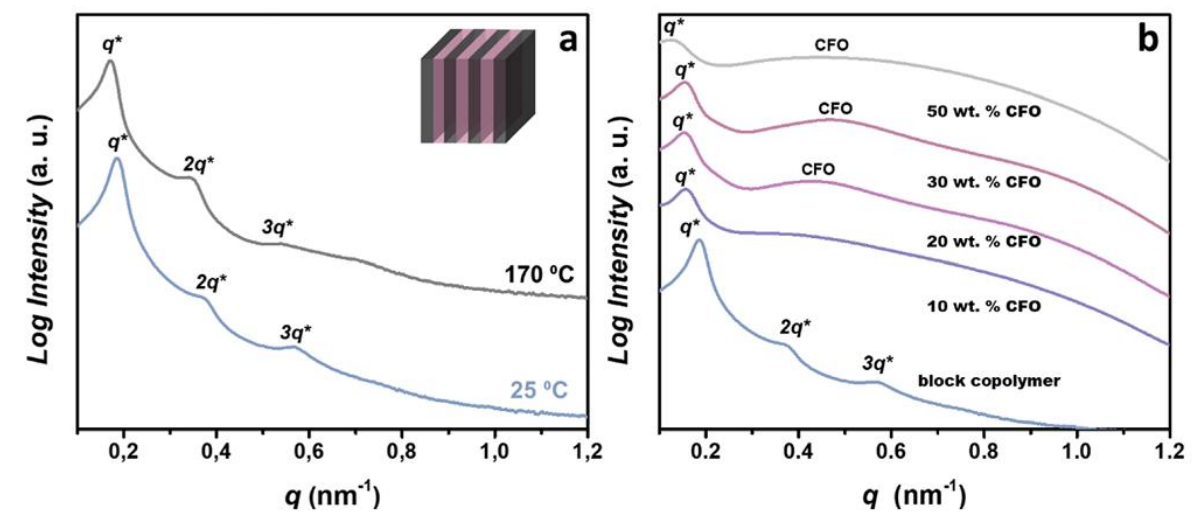

Figure 40. (a) SAXS profile for a P2VP-b-poly(VDF-co-TrFE)- $b$-P2VP block copolymer collected at $170{ }^{\circ} \mathrm{C}$ and at room temperature showing the formation of the lamellar structure in the melt and its preservation after the crystallization of the poly(VDF-co-TrFE) copolymers. (b) 
SAXS profiles of block copolymer/cobalt ferric oxide nanocomposites at different loading of nanoparticles.[277] Reproduced with permission from Royal Society of Chemistry.

\subsection{Biomedical applications}

The applications of fluorinated polymers as smart materials for advanced biomedical devices (stents, suture wires, implants, etc.) has recently been reviewed by Lanceros-Méndez's team.[15] Fluorinated polymers bearing precisely placed functionalities and displaying stimuli-responsive properties, such as electroactive (ferroelectric, pyroelectric and, in particular, piezoelectric features),[278] i.e., its ability to convert electrical stimuli into mechanical response, and vice versa. These properties have been increasingly investigated and applied in the biomedical field to develop highly instrumented devices, artificial muscle actuators, advanced tissue engineering and drugs delivery approaches.[15]

An efficient surface functionalization of poly(TFE-co-HFP) copolymer (FEP) by ion beam-based surface grafting enabled to produce a new platform for fluorescence-based bioassays (Figure 41). Jung et al.[279] developed the graft polymerization of acrylic acid (AA) thanks to the carboxylic acid groups on the surface of FEP, well evidenced by surface analysis. These resulting FEP- $g$ PAA could find some uses in the detection of disease-related target biomolecules, anthrax toxin pDNA and a liver cancer specific target AFP antigen that could be immobilized on the PAA branches. The p-DNA-immobilized FEP surface allowed detection of the target DNA with a concentration of as low as $10 \mathrm{fg} / \mathrm{mL}(1.15 \mathrm{fM})$ while the lowest detectable concentration of the target AFP antigen from this method was $10 \mathrm{pg} / \mathrm{mL}$ (142 fM). Such a surface functionalization by graft polymerization may find various other biomedical applications as fluorescence-based bioassays, biochips, and biosensors for the detection of target biomolecules such as DNA, antibodies, antigens, and other biomolecules. 


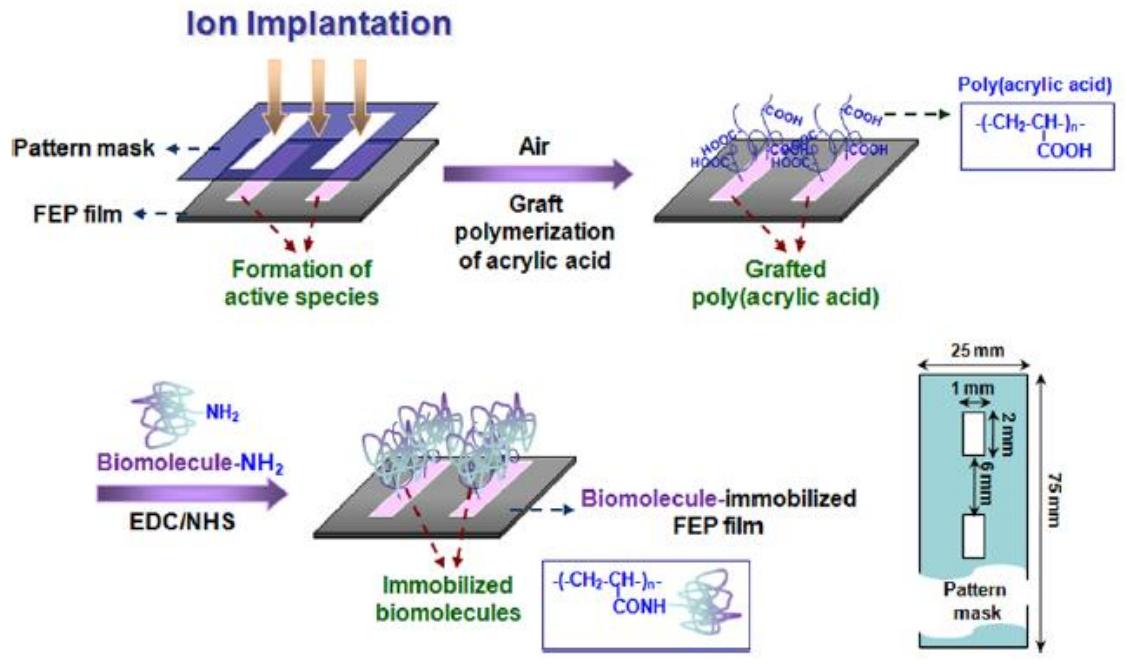

Figure 41. Schematic representation of biomolecular patterning by ion implantation.[279] Reproduced with permission from American Chemical Society.

8.10. Gel polymer electrolyte for Li-ion battery

VDF-based polymers and copolymers exhibit interesting properties for use as separator membranes or polymer electrolytes in lithium-ion battery (LIB) applications.[254-256] Apostolides et al.[240] developed amphiphilic polymer conetworks based on four-arm stars of PVDF and PEO for potential application as solid electrolyte in LIBs (Scheme 65). The PVDF stars were prepared via RAFT polymerization of VDF using a tetraxanthate CTA. 

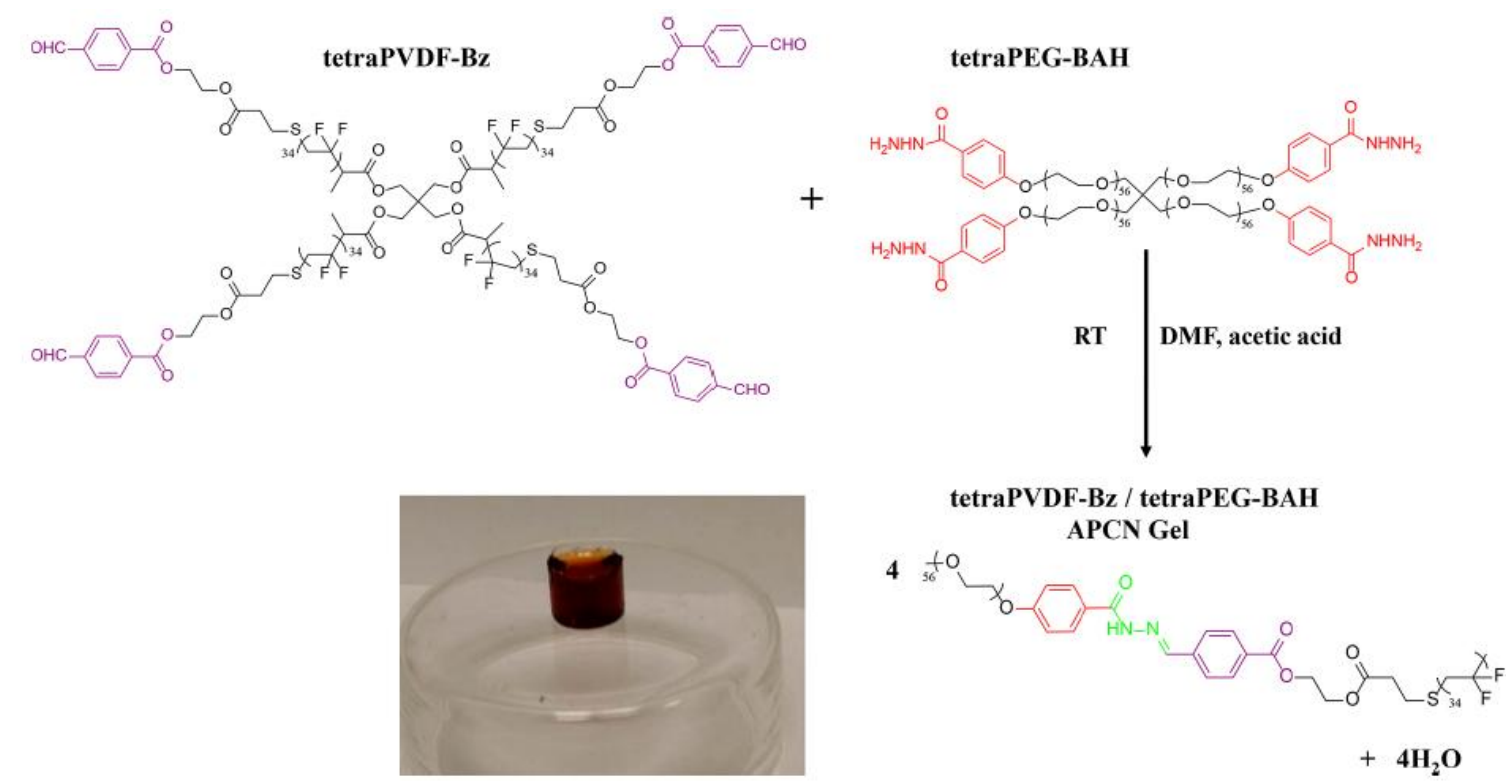

Scheme 65. Condensation Reaction between TetraPVDF-Bz and TetraPEG-BAH, leading to the formation of the APCN Gel, and photograph of the resulting gel.[240] Reproduced with permission from American Chemical Society.

Doped with Li-salts, these original PVDF/PEO system displayed high conductivities (ca. 2.4 $\left.\mathrm{mS} . \mathrm{cm}^{-1}\right)$ and suitable electrochemical stability at high potential.

\subsection{Functional coatings}

Similar to the above strategy, Lopez et al.[239] developed a 4-arm star PVDF (Figure 42) as an appealing alternative to PVDF/PMMA blends since it provides crosslinked PVDF transparent coatings via photocrosslinking as a fast and versatile technique. PVDF tetraxanthate star reacted onto the acrylate end group of AHPMA selectively via a thia-Michael to offer a tetrafunctional methacrylate that homopolymerized or copolymerized with a telechelic diacrylate perfluorooligoether. The produced coating materials displayed excellent adhesion properties onto metal surfaces. Furthermore, their surface energies and water contact angles were tunable. 


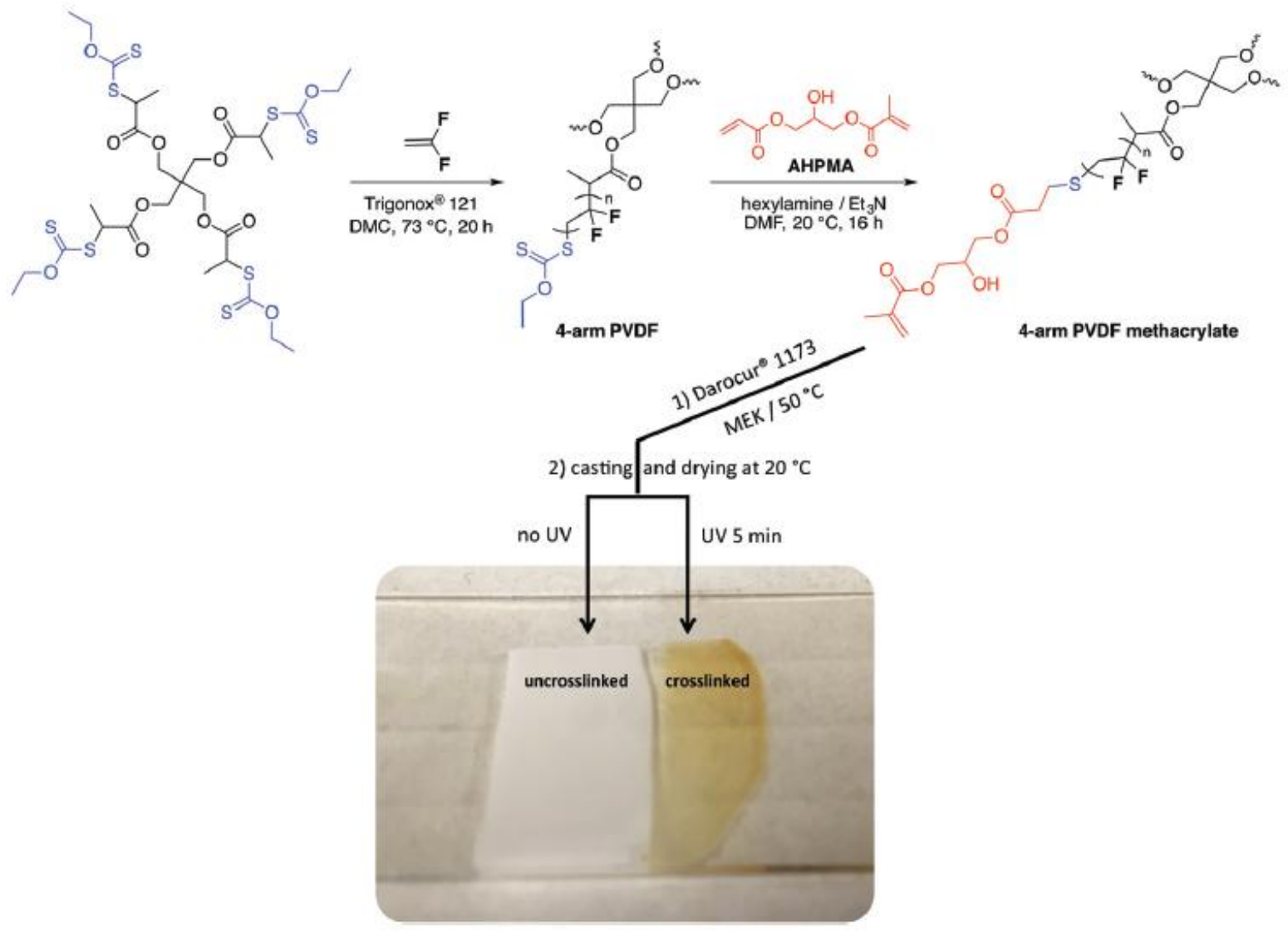

Figure 42. Synthesis and photocrosslinking of 4-arm star PVDF methacrylate prepared via RAFT opolymerization of VDF from a tetra-arm xanthate initiator, followed by thia-Michael addition onto a (meth)acrylate (AHPMA) to produce a tetra-methacrylate PVDF star that copolymerize for specific coating.[239] Reproduced with permission from Royal Society of Chemistry.

Yanai et al.[280] reported the use of carboxylate sites on the pore surface of the porous coordination polymers (PCPs) for the functionalization of oligo(VDF)-I, I-OVDF with terminal alkenes. The rational modification of the PCP pore walls with reactive basic sites allowed for the highly accelerated and efficient elimination reaction of the guest OVDF. However, the authors have not reported the drawback of the well-known base selectivity of VDF units in I-OVDF compounds. 
Durand et al.[275] deeply investigated the compatibility behavior of nanoparticles surface with fluorinated matrices in order to prepare a homogenous dispersion and better composites properties. First, the authors prepared $\mathrm{C}_{6} \mathrm{~F}_{13} \mathrm{I}$ (Scheme 64) and/or $\mathrm{C}_{6} \mathrm{~F}_{13}-\mathrm{C}_{2} \mathrm{H}_{4}-\mathrm{SH}$ modified silica nanoparticles. Thus prepared modified silica were mixed with molten poly(VDF-co-HFP) and poly(TFE-co-HFP) to prepare polymer matrices with improved hydrophobic, mechanical, and thermal properties.

\subsection{Shape memory polymers}

Shape memory polymers (SMPs) enable to switch between a temporary and a permanent shape under the influence of an external stimuli (temperature, $\mathrm{pH}$, electrical, stress, magnetic effect, etc.).[281, 282] Eken and Acar[283] synthesized poly(VDF-co-CTFE)-g-PEOMA graft copolymers with tunable mechanical and thermal properties. They highlighted two melting points $\left(32-37{ }^{\circ} \mathrm{C}\right.$ and $166{ }^{\circ} \mathrm{C}$ ), assigned to PEOMA and poly(VDF-co-CTFE) copolymers, respectively, and shape memory properties under temperature as potential clinical devices, wires and implants. However, no information on $\alpha$ and $\beta$ phases of PVDF was supplied.

\subsection{Conclusions}

Despite those advances which demonstrate the strong potential of fluoropolymers involved in multiple applications, there is still a long path to cross to produce specific tailored properties that will fulfil some of the most challenging technological applications in the near future, both in materials and biomedical field. A growing interest is noted from the ATRP grafting of functional hydrocarbon monomers from poly(VDF-co-CTFE) copolymers to prepare fuel cell membranes, binders/separators for Li-ion batteries, antifouling and antimicrobial coatings. Comparing the electrochemical properties of the fuel cell membranes revealed that graft copolymers displayed higher conductivities than the block copolymers. Amphiphilic block copolymers could self- 
assemble to lead to peculiar morphologies, but so far no real applications have been observed. TPEs, thanks to their low temperature resilience and preserving good properties over a wide range of temperatures are still regarded as key high performance materials. Macromolecular architectures based on $\operatorname{TrFE}$ for electroactive materials are graft copolymers achieved from ATRP of styrene and ethyl methacrylate from poly(VDF-ter-TrFE-ter-CTFE) terpolymers. The primary issue is the small amount of available electroactive fluorinated copolymers with active sites homogeneously ranked along the polymer chain. The study of architectured EAPs is still in its infancy. Major advances in the synthesis of novel fluoropolymer topologies in future could lead to interesting developments and give insights on the effects of nanoconfinement.

\section{Conclusions and Perspectives}

ITP, RAFT/MADIX, CMRP and reactions involving borane seem to be the most suitable techniques of RDRP of F-alkenes and the first one (that is the oldest RDRP technique) which has already been industrially scaled up for various high-tech areas. Understanding of the structureactivity relationship of the fluoromonomer and detailed kinetics and mechanistic parameters of the polymerization reactions will lead to advanced materials based on fluoropolymers for targeted applications. Though many articles report RDRP carried out in solution polymerization, emulsion and suspension (co)polymerizations of such gases have also been deeply studied in industry. However, fluoroolefins are gases, hence requiring specific high-pressure autoclaves of various dimensions, and, though many secrets have been carefully kept by industries, there are still many opportunities to pursue such a fascinating research area. More recent works on CMRP seem challenging and encouraging, though the heavy metals can be an environmental issue. Well-architectured fluoropolymers (block, grafts, alternating, stars and miktoarms) could be 
designed thanks to RDRP and with the suitable initiator, CTA or organometallic complex. A wide range of block copolymers has been developed only by ITP or RAFT but these techniques related to transfer reactions have obviously led to lower molar masses. Graft copolymers involving PVDF as macroinitiator or mainly based on the $\mathrm{C}-\mathrm{Cl}$ cleavage in CTFE units offer wide opportunities of materials devoted to modern applications (Energy, antifungal or antimicrobial coatings and water treatment).

RDRP of fluoroalkenes has shown a growing interest since several commercially available products have been on the market from early 80ies. While homopolymers have been controlled (VDF leading to the most consistent results), several examples of copolymers have been reported especially for CTFE and TrFE because the corresponding homopolymers are not soluble in solvents commonly used for SEC and thus would hamper their molar mass and dispersity assessments. The choice of the comonomer partner depends on its reactivity versus that of the fluoromonomer. Surprisingly, though PTFE is the most produced fluoropolymer under conventional radical polymerization, quite a few articles have reported RDRP of TFE. Such high value-added materials should satisfy a broad range of demands in term of mechanical and thermal properties to be used in harsh conditions. The interest in products manufactured using such techniques is expectedly to grow as fast as the fluoropolymer industry. In spite of more recent appealing domains such as self-healing materials, it can be expected further studies in that direction. Again, fluoropolymers are unique materials that fulfil a wide range of stringent requirements and it is expected that the discovery of new products, properties, and applications will grow immensely in the coming years.

\section{Acknowledgements}


The authors thank postdoc researchers and $\mathrm{PhD}$ students (mentioned as co-authors in the list of references), and industrial companies and colleagues for fruitful discussions and for building valuable collaborations and/or for sponsoring various studies and/or supplying free samples, as well as the French National Agency (ANR PREMHYS and FLUPOL projects) and the Council of Scientific and Industrial Research, New Delhi, India for financial support. SS and SAM thank DBT and MHRD for their fellowships, respectively. SB thanks SERB, Govt. of India for the Ramanujan Fellowship Award (SB/S2/RJN-113/2016) and Early Career Research Award (ECR/2018/001990). SB thanks IIT Bhilai for Research Initiation Grant while the French Fluorine Network is also acknowledged.

\section{References}

[1] Ren JM, McKenzie TG, Fu Q, Wong EHH, Xu J, An Z, Shanmugam S, Davis TP, Boyer C, Qiao GG. Star Polymers. Chem Rev. 2016;116:6743-836.

[2] Ebnesajjad S, editor. Fluoroplastics: melt processible fluoropolymersthedefinitive user's guide and databook, vol. 2. Norwich NY: Plastics Design Library; 2003, 766 pp.

[3] Scheirs J, editor. Modern Fluoropolymers: High Performance Polymers for Diverse Applications. New York: Wiley Interscience;1997. p. 435-85.

[4] Hougham G, Cassidy PE, Johns K, Davidson J, editors. Fluoropolymers:synthesis and applications. New York: Plenum Publish; 1999, 329 pp.

[5] Améduri B, Boutevin B, editors. Well-architectured fluoropolymers: synthesis,properties and applications. Oxford: Elsevier Ltd; 2004, 508 pp.

[6] Ameduri B, Sawada H, editors. Fluoropolymers: From Fundamentals to Applications. Oxford: Royal Society of Chemistry; 2016, Volume 1 (p. 392) and Volume 2 (p. 372). 
[7] Ameduri B, Boutevin B. Update on fluoroelastomers: from perfluoroelastomers to fluorosilicones and fluorophosphazenes. J Fluorine Chem. 2005;126:221-9.

[8] Moore AL. Fluoroelastomers handbook. Norwich, NY: William Andrew Inc; 2005, 373 pp.

[9] Smith Jr DW, Iacono ST, Iyer SS. Handbook of Fluoropolymer Science andTechnology. New York: John Wiley \& Sons Inc; 2014, 672 pp.

[10] Desbief S, Grignard B, Detrembleur C, Rioboo R, Vaillant A, Seveno D, Voué M, Coninck J d, Jonas A M, Jérôme C, Damman P, Lazzaroni R. Superhydrophobic Aluminum Surfaces by Deposition of Micelles of Fluorinated Block Copolymers. Langmuir. 2010;26:2057-67. [11] Licchelli M, Malagodi M, Weththimuni ML, Zanchi C. Water-repellent properties of fluoroelastomers on a very porous stone: Effect of the application procedure. Prog Org Coat. 2013;76:495-503.

[12] DeBergalis M. Fluoropolymer films in the photovoltaic industry. J Fluorine Chem. 2004;125:1255-7.

[13] Meyer F. Fluorinated conjugated polymers in organic bulk heterojunction photovoltaic solar cells. Prog Polym Sci. 2015;47:70-91.

[14] Zhang Z, Shen W, Ling J, Yan Y, Hu J, Cheng Y. The fluorination effect of fluoroamphiphiles in cytosolic protein delivery. Nat Commun. 2018;9:1377.

[15] Cardoso V, Correia D, Ribeiro C, Fernandes M, Lanceros-Méndez S. Fluorinated Polymers as Smart Materials for Advanced Biomedical Applications. Polymers. 2018;10:161.

[16] Seymour RB, Kirshenbaum GS. History of High Performance Polymers. New York, USA: Elsevier; 1987, $461 \mathrm{pp}$.

[17] Puts GJ, Crouse P, Ameduri BM. Polytetrafluoroethylene: Synthesis and Characterization of the Original Extreme Polymer. Chem Rev. 2019;119:1763-805. 
[18] Taguet A, Ameduri B, Boutevin B. Crosslinking of Vinylidene Fluoride-Containing Fluoropolymers. Adv Polym Sci. 2005;184:127-211.

[19] Humphrey JS, Amin-Sanayei R. Vinylidene Fluoride Polymers. In: Mark HF, editor. Encyclopedia of Polymer Science and Technology. 3rd ed, 2004. p. 510-33.

[20] Ameduri B. From Vinylidene Fluoride (VDF) to the Applications of VDF-Containing Polymers and Copolymers: Recent Developments and Future Trends. Chem Rev. 2009;109:6632-86.

[21] Ameduri B. Controlled Radical (Co)polymerization of Fluoromonomers. Macromolecules. 2010;43:10163-84.

[22] Banerjee S, Paira TK, Mandal TK. Surface confined atom transfer radical polymerization: access to custom library of polymer-based hybrid materials for speciality applications. Polym Chem. 2014;5:4153-67.

[23] Andruzzi L, Hexemer A, Li X, Ober CK, Kramer EJ, Galli G, Chiellini E, Fischer DA. Control of Surface Properties Using Fluorinated Polymer Brushes Produced by Surface-Initiated Controlled Radical Polymerization. Langmuir. 2004;20:10498-506.

[24] Satoh K, Kamigaito M. Stereospecific Living Radical Polymerization: Dual Control of Chain Length and Tacticity for Precision Polymer Synthesis. Chem Rev. 2009;109:5120-56. [25] Rosen BM, Percec V. Single-Electron Transfer and Single-Electron Transfer Degenerative Chain Transfer Living Radical Polymerization. Chem Rev. 2009;109:5069-119.

[26] Matyjaszewski K. Atom Transfer Radical Polymerization (ATRP): Current Status and Future Perspectives. Macromolecules. 2012;45:4015-39.

[27] Monteiro MJ, Cunningham MF. Polymer Nanoparticles via Living Radical Polymerization in Aqueous Dispersions: Design and Applications. Macromolecules. 2012;45:4939-57. 
[28] Tsarevsky NV, Sumerlin BS, editors. Fundamentals of Controlled/Radical Polymerization. Oxford, UK: Royal Society of Chemistry; 2013.

[29] Hansen NML, Jankova K, Hvilsted S. Fluoropolymer materials and architectures prepared by controlled radical polymerizations. Eur Polym J. 2007;43:255-93.

[30] Destarac M. Industrial development of reversible-deactivation radical polymerization: is the induction period over? Polym Chem. 2018;9:4947-67.

[31] Tatemoto M, Nakagawa T. Segmented polymers. German Patent 2,729,671 (assigned to Daikin Kogyo Co. Ltd); 1978.

[32] Tatemoto M. The first Regular Meeting of Soviet-Japanese Fluorine Chemists. Tokyo1979. [33] Tatemoto M, Shimizu T. Thermoplastic Elastomers, . In: Scheirs J, editor. Modern Fluoropolymers, Chapter 25. New-York: Wiley; 1997. p. 565-76.

[34] Fu GD, Kang ET, Neoh KG, Lin CC, Liaw DJ. Rigid Fluorinated Polyimides with WellDefined Polystyrene/Poly(pentafluorostyrene) Side Chains from Atom Transfer Radical Polymerization. Macromolecules. 2005;38:7593-600.

[35] Eberhardt M, Théato P. RAFT Polymerization of Pentafluorophenyl Methacrylate:

Preparation of Reactive Linear Diblock Copolymers. Macromol Rapid Commun. 2005;26:148893.

[36] Inoue Y, Watanabe J, Takai M, Yusa S-i, Ishihara K. Synthesis of sequence-controlled copolymers from extremely polar and apolar monomers by living radical polymerization and their phase-separated structures. J Polym Sci A Polym Chem. 2005;43:6073-83.

[37] Matyjaszewski K, Spanswick J. Controlled/living radical polymerization. Materials Today. 2005;8:26-33.

[38] Wlassics I, Rapallo G, Apostolo M, Bellinzago N, Albano M. EP1999/0979832A1 
(assigned to Ausimont S.p.A.); 1990.

[39] Yagi T, Tatemoto M. A Fluorine-19 NMR Study of the Microstructure of Vinylidene Fluoride-Trifluoroethylene Copolymers. Polym J. 1979;11:429-36.

[40] Otsu T, Yoshida M. Role of initiator-transfer agent-terminator (iniferter) in radical polymerizations: Polymer design by organic disulfides as iniferters. Macromol Rapid Commun. $1982 ; 3: 127-32$

[41] Otsu T, Matsumoto A. Iniferter concept and living radical polymerization. Adv Polym Sci. 1998;136:75.

[42] Nicolas J, Guillaneuf Y, Lefay C, Bertin D, Gigmes D, Charleux B. Nitroxide-mediated polymerization. Prog Polym Sci. 2013;38:63-235.

[43] David G, Boyer C, Tonnar J, Ameduri B, Lacroix-Desmazes P, Boutevin B. Use of iodocompounds in radical polymerization. Chem Rev. 2006;106:3936-62.

[44] Hill MR, Carmean RN, Sumerlin BS. Expanding the scope of RAFT polymerization: recent advances and new horizons. Macromolecules. 2015;48:5459-69.

[45] Perrier S. 50th Anniversary Perspective: RAFT Polymerization-A User Guide. Macromolecules. 2017;50:7433-47.

[46] Rizzardo E, Chiefari J, Mayadunne RTA, Moad G, Thang SH. Synthesis of Defined Polymers by Reversible Addition Fragmentation Chain Transfer: The RAFT Process. ACS Symp Ser. 2000;768:278-96.

[47] Destarac M. Controlled radical polymerization: industrial stakes, obstacles and achievements. Macromol React Eng. 2010;4:165-79.

[48] Yamago S. Precision polymer synthesis by degenerative transfer controlled/living radical polymerization using organotellurium, organostibine, and organobismuthine chain-transfer 
agents. Chem Rev. 2009;109:5051-68.

[49] Demarteau J, Debuigne A, Detrembleur C. Organocobalt Complexes as Sources of CarbonCentered Radicals for Organic and Polymer Chemistries. Chem Rev. 2019;119:6906-55.

[50] Chung TCM. Synthesis of Fluoropolymers Using Borane-Mediated Control Radical Polymerization for Energy Storage Applications. In: Smith DW, Iacono ST, Iyer SS, editors. Handbook of Fluoropolymer Science and Technology. p. 291-314.

[51] Lin W, Niu H, Chung TCM, Dong J-Y. Borane chain transfer reaction in olefin polymerization using trialkylboranes as chain transfer agents. J Polym Sci, Part A: Polym Chem. 2010;48:3534-41.

[52] Goto A, Suzuki T, Ohfuji H, Tanishima M, Fukuda T, Tsujii Y, Kaji H. Reversible Complexation Mediated Living Radical Polymerization (RCMP) Using Organic Catalysts. Macromolecules. 2011;44:8709-15.

[53] Goto A, Ohtsuki A, Ohfuji H, Tanishima M, Kaji H. Reversible Generation of a CarbonCentered Radical from Alkyl Iodide Using Organic Salts and Their Application as Organic Catalysts in Living Radical Polymerization. J Am Chem Soc. 2013;135:11131-9.

[54] Timmerman R, Greyson W. The predominant reaction of some fluorinated polymers to ionizing radiation. J Appl Polym Sci. 1962;6:456-60.

[55] Lacroix-Desmazes P, Ameduri B, Boutevin B. Use of fluorinated organic compounds in living radical polymerizations. Collect Czech Chem Commun. 2002;67:1383-415.

[56] Ameduri B. Recent advances in the controlled radical (co) polymerization of fluoroalkenes and applications therefrom. J Taiwan Inst Chem Eng. 2014;45:3124.

[57] Banerjee S, Chakrabarty A, Singha NK, Ameduri A. Recent Advances in the Reversible Deactivation Radical (Co)Polymerisation of Fluorinated Alkenes/Acrylates/ Methacrylates/ 
Styrenes. In: Singha NK, Mays JW, editors. Functional Polymers by Reversible Deactivation Radical Polymerisation: Synthesis and Applications: Smithers Rapra Technology; 2017. [58] Hawker CJ, Bosman AW, Harth E. New Polymer Synthesis by Nitroxide Mediated Living Radical Polymerizations. Chem Rev. 2001;101:3661-88.

[59] Banerjee S, Domenichelli I, Ameduri B. Nitroxide-Mediated Alternating Copolymerization of Vinyl Acetate with tert-Butyl-2-trifluoromethacrylate Using a SG1-Based Alkoxyamine. ACS Macro Lett. 2016;5:1232-6.

[60] Braunecker WA, Matyjaszewski K. Controlled/living radical polymerization: Features, developments, and perspectives. Prog Polym Sci. 2007;32:93-146.

[61] Kamigaito M, Ando T, Sawamoto M. Metal-Catalyzed Living Radical Polymerization. Chem Rev. 2001;101:3689-746.

[62] Poli R. Relationship between One-Electron Transition-Metal Reactivity and Radical Polymerization Processes. Angew Chem Int Ed. 2006;45:5058-70.

[63] Boutevin B. From telomerization to living radical polymerization. J Polym Sci A Polym Chem. 2000;38:3235-43.

[64] Apostolo M, Arcella V, Storti G, Morbidelli M. Free Radical Controlled Polymerization of Fluorinated Copolymers Produced in Microemulsion. Macromolecules. 2002;35:6154-66.

[65] Oka M, Tatemoto M. Vinylidene fluoride-hexafluoropropylene copolymer having terminal iodines. In: Bailey WJ, Tsuruta T, editors. Contemporary Topics in Polymer Science. New-York: Plenun Press; 1984. p. 763-81.

[66] Tatemoto M. Fluorinated thermoplastic elastomers. Int Polym Sci Technol. 1985;12:85-91 (translated into English from Nippon Gomu Kyaokaishi, 1984, 57, 761-767).

[67] Boyer C, Valade D, Sauguet L, Ameduri B, Boutevin B. Iodine Transfer Polymerization 
(ITP) of Vinylidene Fluoride (VDF). Influence of the Defect of VDF Chaining on the Control of ITP. Macromolecules. 2005;38:10353-62.

[68] Arcella V, Albano M, Barchiesi E, Brinati G, Chiodini G. Rubber World. 1993;207:18.

[69] Boyer C, Valade D, Lacroix-Desmazes P, Ameduri B, Boutevin B. Kinetics of the iodine transfer polymerization of vinylidene fluoride. J Polym Sci A Polym Chem. 2006;44:5763-77.

[70] Beuermann S, Imran-ul-Haq M. Homogeneous Phase Polymerization of Vinylidene

Fluoride in Supercritical CO2: Surfactant Free Synthesis and Kinetics. Macromol Symp. 2007;259:210-7.

[71] Imran-ul-Haq M, Förster N, Vukicevic R, Herrmann K, R. S, Beuermann S. ACS Symposium Series. 2009;11.

[72] DeSimone JM, Maury EE, Menceloglu YZ, McClain JB, Romack TJ, Combes JR. Dispersion Polymerizations in Supercritical Carbon Dioxide. Science. 1994;265:356.

[73] Gregory A, Stenzel MH. Complex polymer architectures via RAFT polymerization: From fundamental process to extending the scope using click chemistry and nature's building blocks. Prog Polym Sci. 2012;37:38-105.

[74] Moad G. A Critical Survey of Dithiocarbamate Reversible Addition- Fragmentation Chain Transfer (RAFT) Agents in Radical Polymerization. J Polym Sci A Polym Chem. 2019;57:21627.

[75] Matioszek D, Mazières S, Brusylovets O, Lin CY, Coote ML, Destarac M, Harrisson S. Experimental and Theoretical Comparison of Addition-Fragmentation Pathways of Diselenoand Dithiocarbamate RAFT Agents. Macromolecules. 2019;52:3376-86.

[76] Haven JJ, Hendrikx M, Junkers T, Leenaers PJ, Tsompanoglou T, Boyer C, Xu J, Postma A, Moad G. Elements of RAFT Navigation. Reversible Deactivation Radical Polymerization: 
Mechanisms and Synthetic Methodologies: American Chemical Society; 2018. p. 77-103.

[77] Brandl F, Drache M, Beuermann S. Kinetic Monte Carlo Simulation Based Detailed Understanding of the Transfer Processes in Semi-Batch Iodine Transfer Emulsion Polymerizations of Vinylidene Fluoride. Polymers. 2018;10:1008.

[78] Banerjee S, Patil Y, Ono T, Ameduri B. Synthesis of w-Iodo and Telechelic Diiodo Vinylidene Fluoride-Based (Co)polymers by Iodine Transfer Polymerization Initiated by an Innovative Persistent Radical. Macromolecules. 2017;50:203-14.

[79] Asandei AD, Adebolu OI, Simpson CP. Mild-Temperature Mn2(CO)10-Photomediated Controlled Radical Polymerization of Vinylidene Fluoride and Synthesis of Well-Defined Poly(vinylidene fluoride) Block Copolymers. J Am Chem Soc. 2012;134:6080-3.

[80] Asandei AD, Adebolu OI, Simpson CP, Kim J-S. Visible-Light Hypervalent Iodide Carboxylate Photo(trifluoro)methylations and Controlled Radical Polymerization of Fluorinated Alkenes. Angew Chem Int Ed. 2013;52:10027-30.

[81] Simpson CP, Adebolu OI, Kim J-S, Vasu V, Asandei AD. Metal and Ligand Effects of Photoactive Transition Metal Carbonyls in the Iodine Degenerative Transfer Controlled Radical Polymerization and Block Copolymerization of Vinylidene Fluoride. Macromolecules. 2015;48:6404-20.

[82] Le TP, Moad G, Rizzardo E, Thang SH. Polymerization with living characteristics with controlled dispersity, polymers prepared thereby, and chain-transfer agents used in the same. WO9801478A1 (assigned to E. I. Du Pont de Nemours \& Co., USA); 1998.

[83] Kostov G, Boschet F, Buller J, Badache L, Brandsadter S, Ameduri B. First Amphiphilic Poly(vinylidene fluoride-co-3,3,3-trifluoropropene)-b-oligo(vinyl alcohol) Block Copolymers as Potential Nonpersistent Fluorosurfactants from Radical Polymerization Controlled by Xanthate. 
Macromolecules. 2011;44:1841-55.

[84] Guerre M, Campagne B, Gimello O, Parra K, Ameduri B, Ladmiral V. Deeper Insight into the MADIX Polymerization of Vinylidene Fluoride. Macromolecules. 2015;48:7810-22.

[85] Guerre M, Lopez G, Soulestin T, Totée C, Améduri B, Silly G, Ladmiral V. A Journey into the Microstructure of PVDF Made by RAFT. Macromol Chem Phys. 2016;217:2275-85.

[86] Guerre M, Rahaman SMW, Ameduri B, Poli R, Ladmiral V. Limits of Vinylidene Fluoride RAFT Polymerization. Macromolecules. 2016;49:5386-96.

[87] Guerre M, Uchiyama M, Folgado E, Semsarilar M, Améduri B, Satoh K, Kamigaito M,

Ladmiral V. Combination of Cationic and Radical RAFT Polymerizations: A Versatile Route to Well-Defined Poly(ethyl vinyl ether)-block-poly(vinylidene fluoride) Block Copolymers. ACS Macro Lett. 2017;6:393-8.

[88] Yang Q, Guerre M, Ladmiral V, Ameduri B. Thermal and photo-RAFT polymerization of 2,2,2-trifluoroethyl $\alpha$-fluoroacrylate. Polym Chem. 2018;9:3388-97.

[89] Yang Q, Ladmiral V, Ameduri BM. PhotoRAFT polymerization of vinylidene fluoride using household white LED as light source at room temperature. Chem Photo Chem. 2019;doi:10.1002/cptc.201900139

[90] Natta G. Process involving the reaction of group i-b metal compounds with an organoboron compound in the presence of a strong base. US3134823A. 1960.

[91] Sianesi D, Caporiccio G. Polymerization and copolymerization studies on vinyl fluoride. J Polym Sci, Part A: Polym Chem. 1968;6:335-52.

[92] Zhang Z-C, Chung TCM. Borane-mediated radical polymerization; Preparation of Fluoropolymers for High Energy Density Capacitors. Controlled/Living Radical Polymerization: Progress in RAFT, DT, NMP \& OMRP: American Chemical Society; 2009. p. 331-44. 
[93] Zhang Z-C, Wang Z, Chung TCM. Synthesis of Chain End Functionalized Fluoropolymers by Functional Borane Initiators and Application in the Exfoliated Fluoropolymer/Clay Nanocomposites. Macromolecules. 2007;40:5235-40.

[94] Aliwi SM, Bamford CH, Mullik SU. Recent studies in photoinitiated polymerization. J Polym Sci: Polym Symp. 1975;50:33-50.

[95] Morales-Cerrada R, Fliedel C, Daran J-C, Gayet F, Ladmiral V, Améduri B, Poli R. Fluoroalkyl Radical Generation by Homolytic Bond Dissociation in Pentacarbonylmanganese Derivatives. Chem Eur J. 2019;25:296-308.

[96] Morales-Cerrada R. Complexes de manganèse pentacarbonyle alkyle et fluoroalkyle comme modèles d'espèces dormantes de l'OMRP. Toulouse: University of Toulouse; 2018.

[97] Banerjee S, Ladmiral V, Debuigne A, Detrembleur C, Poli R, Améduri B.

Organometallic- Mediated Radical Polymerization of Vinylidene Fluoride. Angew Chem Int Ed. 2018;57:2934-7.

[98] Falireas PG, Ladmiral V, Debuigne A, Detrembleur C, Poli R, Ameduri B. Straightforward Synthesis of Well-Defined Poly(vinylidene fluoride) and Its Block Copolymers by CobaltMediated Radical Polymerization. Macromolecules. 2019;52:1266-76.

[99] Poli R, Rahaman SMW, Ladmiral V, Ameduri B. Effect of $\alpha$ - and $\beta-\mathrm{H} / \mathrm{F}$ substitution on the homolytic bond strength in dormant species of controlled radical polymerization: OMRP vs. ITP and RAFT. J Organomtal Chem. 2018;864:12-8.

[100] Simoes JAM, Beauchamp JL. Transition metal-hydrogen and metal-carbon bond strengths: the keys to catalysis. Chem Rev. 1990;90:629-88.

[101] Tatemoto M. Polymeric Materials Encyclopedia. In: Salamone JC, editor.: CRC Boca Raton; 1996. Volume 5, p. 3847-62. 
[102] Tatemoto M, Furukawa Y, Tomoda M, Oka M, Morita S. Fluorine-containing polymer and composition containing the same. EP2103636B1. (assigned to Daikin); 1980.

[103] Tatemoto M, Morita S. Liquid fluoropolymer rubber. Eur. Pat. Appl. EP 27,721(assigned to Daikin); 1981.

[104] Apostolo M, Arcella V, Storti G, Morbidelli M. Kinetics of the Emulsion Polymerization of Vinylidene Fluoride and Hexafluoropropylene. Macromolecules. 1999;32:989-1003.

[105] Boyer C, Ameduri B. Iodine Transfer Copolymerization of Vinylidene Fluoride and $\alpha$ Trifluoromethacrylic Acid in Emulsion Process without any Surfactants. J Polym Sci Part A: Polym Chem. 2009;47:4710-22.

[106] Ameduri B, Boyer C. Transparent vinylidene fluoride-based fluoropolymers and their manufacture by emulsion polymerization without using emulsifiers. JP2008214420A (assigned to Tosoh F-Tech Inc., Japan); 2008.

[107] Banerjee S, Schmidt J, Talmon Y, Hori H, Asai T, Ameduri B. A degradable fluorinated surfactant for emulsion polymerization of vinylidene fluoride. Chem Commun. 2018;54:11399402.

[108] Boyer C, Ameduri B, Boutevin B, Dolbier WR, Winter R, Gard G. Radical Terpolymerization of 1,1,2-Trifluoro-2-pentafluorosulfanylethylene and Pentafluorosulfanylethylene in the Presence of Vinylidene Fluoride and Hexafluoropropylene by Iodine Transfer Polymerization. Macromolecules. 2008;41:1254-63.

[109] Boyer C, Ameduri B, Hung MH. Telechelic Diiodopoly(VDF-co-PMVE) Copolymers by Iodine Transfer Copolymerization of Vinylidene Fluoride (VDF) with Perfluoromethyl Vinyl Ether (PMVE). Macromolecules. 2010;43:3652-63.

[110] Hung M-H, Ameduri B, Boyer CAJ-M. Difunctional oligomers of perfluoro(methyl vinyl 
ether). WO2009055521A1 (assigned to DuPont Performance Elastomers L.L.C., USA); 2009. [111] Kostov G, Holan M, Ameduri B, Hung MH. Synthesis and Characterizations of PhotoCross-Linkable Telechelic Diacrylate Poly(vinylidene fluoride-co-perfluoromethyl vinyl ether) Copolymers. Macromolecules. 2012;45:7375-87.

[112] Hung M-H, Ameduri B, Kostov G. Fluoropolymer having diacrylate ends. US Patent US2011/0,015,358 US2013/0072635 (assigned to Dupont Performance Elastomers and CNRS); 2011.

[113] Soules A, Ameduri B, Boutevin B, Calleja G. Original Fluorinated Copolymers Achieved by Both Azide/Alkyne "Click" Reaction and Hay Coupling from Tetrafluoroethylene Telomers. Macromolecules. 2010;43:4489-99.

[114] Hung M-H, Ameduri B, Kostov G. Crosslinking of telechelic diacrylates. US2013/0072637 (assigned to Dupont Performance Elastomers and CNRS); 2013. [115] Hung M-H, Ameduri B. Telechelic triethoxysilanes:Process for preparation of fluorosilicon polymer. US2012/8138274 (assigned to Dupont Performance Elastomers and CNRS); 2013.

[116] Mladenov G, Ameduri B, Kostov G, Mateva R. Synthesis and characterization of fluorinated telomers containing vinylidene fluoride and hexafluoropropene from 1,6diiodoperfluorohexane. J Polym Sci A Polym Chem. 2006;44:1470-85. [117] Durand N, Ameduri B, Boutevin B. Synthesis and characterization of functional fluorinated telomers. J Polym Sci, Part A: Polym Chem. 2011;49:82-92.

[118] Banerjee S, Zaghloul S, Alaaeddine A, Ameduri B. Kinetics and Mechanistic Aspects of the Iodine Transfer Copolymerization of Vinylidene Fluoride with 2,3,3,3-Tetrafluoro-1-propene and Functionalization into w-Hydroxy Fluorinated Copolymer. Polym Chem. 2016;7:6099-109. 
[119] Jaye JA, Sletten EM. Modular and Processable Fluoropolymers Prepared via a Safe, Mild, Iodo-Ene Polymerization. ACS Cent Sci. 2019;5:982-91.

[120] Monteiro MJ, Adamy MM, Leeuwen BJ, van Herk AM, Destarac M. A “Living” Radical ab Initio Emulsion Polymerization of Styrene Using a Fluorinated Xanthate Agent.

Macromolecules. 2005;38:1538-41.

[121] Kostov G, Boschet F, Brandstadter S, Ameduri B. Random and sequential radical cotelomerizations of 3,3,3-trifluoropropene $\left(\mathrm{H}_{2} \mathrm{C}=\mathrm{CHCF}_{3}\right)$ with vinylidene fluoride $\left(\mathrm{F}_{2} \mathrm{C}=\mathrm{CH}_{2}\right)$. J Polym Sci Part A Polym Chem. 2009;47:3964-81.

[122] Patil Y, Ameduri B. First RAFT/MADIX radical copolymerization of tert-butyl 2trifluoromethacrylate with vinylidene fluoride controlled by xanthate. Polym Chem. 2013;4:2783-99.

[123] Ameduri B, Patil Y. Copolymerisation radicalaire controlee de monomers fluores par un xanthate ou trithiocarbonate. US2014/0154611 (assigned to Arkema and CNRS); 2014. [124] Ameduri B, Alaaeddine A. Controlled free-radical copolymerization of trifluoroethylene US2014/0154611 (assigned to Arkema and CNRS); 2014.

[125] Liu L, Lu D, Wang H, Dong Q, Wang P, Bai R. Living/controlled free radical copolymerization of chlorotrifluoroethene and butyl vinyl ether under 60Co [gamma]-ray irradiation in the presence of S-benzyl O-ethyl dithiocarbonate. Chem Commun. 2011;47:783941.

[126] Wang P, Dai J, Liu L, Dong Q, Jin B, Bai R. Xanthate-mediated living/controlled radical copolymerization of hexafluoropropylene and butyl vinyl ether under 60Co [gamma]-ray irradiation and preparation of fluorinated polymers end-capped with a fluoroalkyl sulfonic acid group. Polym Chem. 2013;4:1760-4. 
[127] Puts G, Venner V, Améduri B, Crouse P. Conventional and RAFT Copolymerization of Tetrafluoroethylene with Isobutyl Vinyl Ether. Macromolecules. 2018;51:6724-39.

[128] Demarteau J, Améduri B, Ladmiral V, Mees MA, Hoogenboom R, Debuigne A,

Detrembleur C. Controlled Synthesis of Fluorinated Copolymers via Cobalt-Mediated Radical Copolymerization of Perfluorohexylethylene and Vinyl Acetate. Macromolecules. 2017;50:3750-60.

[129] Thomas WM, O'Shaughnessy MT. Kinetics of chlorotrifluoroethylene polymerization. J polym Sci. 1953;11:455-70.

[130] Murray DL, Harwood HJ, Shendy SMM, Piirma I. The use of sequence distributions to determine monomer feed compositions in the emulsion copolymerization of chlorotrifluoroethylene with vinyl acetate and vinyl propionate. Polymer. 1995;36:3841-8. [131] Baradie B, Shoichet MS. Synthesis of Fluorocarbon-Vinyl Acetate Copolymers in Supercritical Carbon Dioxide: Insight into Bulk Properties. Macromolecules. 2002;35:3569-75. [132] Wang P, Wang H, Dong Q, Bai R. Cobalt-Mediated Radical Copolymerization of Chlorotrifluoroethylene and Vinyl Acetate. Polymers. 2019;11:101.

[133] Debuigne A, Champouret Y, Jerome R, Poli R, Detrembleur C. Mechanistic insights into the cobalt-mediated radical polymerization (CMRP) of vinyl acetate with cobalt(III) adducts as initiators. Chem Eur J. 2008;14:4046-59.

[134] Hurtgen M, Debuigne A, Fustin C-A, Jerome C, Detrembleur C. Organometallic-Mediated Radical Polymerization: Unusual Route toward (Quasi-) Diblock Graft Copolymers Starting from a Mixture of Monomers of Opposed Reactivity. Macromolecules. 2011;44:4623-31. [135] Wang W, Yan D, Bratton D, Howdle SM, Wang Q, Lecomte P. Charge Transfer Complex Inimer: A Facile Route to Dendritic Materials. Adv Mater. 2003;15:1348-52. 
[136] Bates FS, Fredrickson GH. Block copolymer thermodynamics: theory and experiment. Annu Rev Phys Chem. 1990;41:525-57.

[137] Abetz, V, editor. Block Copolymers I: Berlin, Springer; 2005.

[138] Hadjichristidis N, Iatrou H, Pitsikalis M, Pispas S, Avgeropoulos A. Linear and non-linear triblock terpolymers. Synthesis, self-assembly in selective solvents and in bulk. Prog Polym Sci. 2005;30:725-82.

[139] Voet VSD, ten Brinke G, Loos K. Well-defined copolymers based on poly(vinylidene fluoride): From preparation and phase separation to application. J Polym Sci Part A: Polym Chem. 2014;52:2861-77.

[140] Asandei AD. Photomediated Controlled Radical Polymerization and Block Copolymerization of Vinylidene Fluoride. Chem Rev. 2016;116:2244-74.

[141] Valade D, Boyer C, Ameduri B, Boutevin B. Poly(vinylidene fluoride)-b-poly(styrene) Block Copolymers by Iodine Transfer Polymerization (ITP): Synthesis, Characterization, and Kinetics of ITP. Macromolecules. 2006;39:8639-51.

[142] Lopez G, Thenappan A, Améduri B. Synthesis of Chlorotrifluoroethylene-Based Block Copolymers by Iodine Transfer Polymerization. ACS Macro Lett. 2015;4:16-20.

[143] Thenappan A, Ameduri B, Lopez G. Synthesis of Chlorotrifluoroethylene-based Block Copolymers by Iodine Transfer Polymerization. assigned to Honeywell Int. Inc.

[144] Zapsas G, Patil Y, Bilalis P, Gnanou Y, Hadjichristidis N. Poly(vinylidene fluoride)/Polymethylene-Based Block Copolymers and Terpolymers. Macromolecules. 2019;52:1976-84.

[145] Boschet F, Ameduri B. (Co)polymers of Chlorotrifluoroethylene: Synthesis, Properties, and Applications. Chem Rev. 2014;114:927-80. 
[146] Otazaghine B, Sauguet L, Boucher M, Ameduri B. Radical copolymerization of vinylidene fluoride with perfluoroalkylvinyl ethers. Eur Polym J. 2005;41:1747-56.

[147] Ameduri B, Armand M, Boucher M, Boutevin B. Fluoroelastomers with low Tg based on vinylidene fluoride without containing any tetrafluoroethylene or siloxane group. Canadian patent CA 1999/293847; extended WO 2001/49758 (deposited by HYDRO-QUEBEC). [148] Dams R. Fluoropolyether elastomers having low glass transition temperatures. Proceedings of the $17^{\text {th }}$ European Symposium in Fluorine Chemistry. Paris, France, 2013. [149] Černoch P, Petrova S, Černochová Z, Kim J-S, Simpson CP, Asandei AD. $\mathrm{Mn}_{2}(\mathrm{CO})_{10^{-}}$ photomediated synthesis of poly(vinylidene fluoride)- $b$-poly(styrene sulfonate). Eur Polym J. 2015;68:460-70.

[150] McElroy KT, Purrington ST, Bumgardner CL, Burgess JP. Lack of Polymerization of Fluorinated Acrylates. J Fluorine Chem. 1999;95:117-20.

[151] Patil Y, Ameduri B. Advances in the (Co)polymerization of Alkyl 2-

Trifluoromethacrylates and 2-(Trifluoromethyl)acrylic Acid. Prog Polym Sci. 2013;38:703-39.

[152] Golzari N, Adams J, Beuermann S. Inducing $\beta$ Phase Crystallinity in Block Copolymers of Vinylidene Fluoride with Methyl Methacrylate or Styrene. Polymers. 2017;9:306.

[153] Guerre M, Wahidur Rahaman SM, Ameduri B, Poli R, Ladmiral V. RAFT synthesis of well-defined PVDF-b-PVAc block copolymers. Polym Chem. 2016;7:6918-33.

[154] Banerjee S, Patil Y, Gimello O, Ameduri B. Well-defined multiblock poly(vinylidene fluoride) and block copolymers thereof: a missing piece of the architecture puzzle. Chem Commun. 2017;53:10910-3.

[155] Guerre M, Schmidt J, Talmon Y, Améduri B, Ladmiral V. An amphiphilic poly(vinylidene fluoride)- $b$-poly(vinyl alcohol) block copolymer: synthesis and self-assembly in water. Polym 
Chem. 2017;8:1125-8.

[156] Kostov G, Boschet F, Ameduri B. Original Fluorinated Surfactants potentially nonbioaccumulable. J Fluorine Chem. 2009;130:1192-9.

[157] Girard E, Marty J-D, Ameduri B, Destarac M. PhD dissetion: Direct Synthesis of Vinylidene Fluoride-Based Amphiphilic Diblock Copolymers by RAFT/MADIX Polymerization. ACS Macro Lett. 2012;1:270-4.

[158] Girard E. Macromolecular engineering of $\mathrm{CO}_{2}$-philic (co)polymers through RAFT/MADIX polymerization: University of Toulouse; 2012.

[159] Folgado E, Guerre M, Da Costa A, Ferri A, Addad A, Ladmiral V, Semsarilar M. "Onepot" aminolysis/thia-Michael addition preparation of well-defined amphiphilic PVDF-b-PEG-bPVDF triblock copolymers: self-assembly behaviour in mixed solvents. Polym Chem. 2019, DOI: 10.1039/C9PY00970A.

[160] Li L, Li J, Zheng S. Poly(vinylidene fluoride)-block-poly(N-vinylpyrrolidone) diblock copolymers: Synthesis via sequential RAFT/MADIX polymerization and self-assembly behavior. Polymer. 2018;142:61-71.

[161] Uchiyama M, Satoh K, Kamigaito M. Cationic RAFT Polymerization Using ppm Concentrations of Organic Acid. Angew Chem Int Ed. 2015;54:1924-8.

[162] Kovarova A, Svobodova D. Perfluorinated compounds: occurrence and risk profile. Neuro Endocrinol Lett. 2008;29:599-608.

[163] Lindstrom AB, Strynar MJ, Libelo EL. Polyfluorinated Compounds: Past, Present, and Future. Environ Sci Technol. 2011;45:7954-61.

[164] Zaggia A, Ameduri B. Recent advances on synthesis of potentially non-bioaccumulable fluorinated surfactants. Curr Opin Colloid Interface Sci. 2012;17:188-95. 
[165] Ito H, Giese B, Engelbrecht R. Radical reactivity and Q-e values of methyl alpha(trifluoromethyl)acrylate. Macromolecules. 1984;17:2204-5.

[166] Ameduri B, Patil Y. Controlled radical copolymerization by a xanthate Controlled FreeRadical Copolymerization of VDF. US2014/0154611 and US 9,184,461 B2 (assigned by Arkema France, CNRS and l'Ecole Nationale Supérieure de Chimie de Montpellier). [167] Seck S, Bounor-Legare V, Gerard J-F, Ameduri B, Bigarre J, Buvat P. Method for preparing an ion-exchange composite material comprising a polymer matrix and a filler consisting of ion-exchange particles. WO2014/173888 (assigned to CEA, INSA and CNRS); 2013.

[168] Chanthad C, Masser KA, Xu K, Runt J, Wang Q. Synthesis of triblock copolymers composed of poly(vinylidene fluoride-co-hexafluoropropylene) and ionic liquid segments. J Mater Chem. 2012;22:341-4.

[169] Guerre M, Uchiyama M, Lopez G, Ameduri B, Satoh K, Kamigaito M, Ladmiral V. Synthesis of PEVE- $b$-P(CTFE-alt-EVE) block copolymers by sequential cationic and radical RAFT polymerization. Polym Chem. 2018;9:352-61.

[170] Destarac M, Matyjaszewski K, Silverman E, Ameduri B, Boutevin B. Atom Transfer Radical Polymerization Initiated with Vinylidene Fluoride Telomers. Macromolecules. 2000;33:4613-5.

[171] Laruelle G, Nicol E, Ameduri B, Tassin J-F, Ajellal N. Synthesis of poly(vinylidene fluoride)-b-poly(styrene sulfonate) block copolymers by controlled radical polymerizations. J Polym Sci, Part A: Polym Chem. 2011;49:3960-9.

[172] Shi Z, Holdcroft S. Synthesis of Block Copolymers Possessing Fluoropolymer and NonFluoropolymer Segments by Radical Polymerization. Macromolecules. 2004;37:2084-9. 
[173] Zhang Z, Shi Z, Han X, Holdcroft S. Synthesis of Fluorous, Block-Brush Copolymers Containing Vinylidene Fluoride, Styrene, and Ethylene Oxide Blocks. Macromolecules. 2007;40:2295-8.

[174] Xu K, Li K, Khanchaitit P, Wang Q. Synthesis and Characterization of Self-Assembled Sulfonated Poly(styrene-b-vinylidene fluoride-b-styrene) Triblock Copolymers for Proton Conductive Membranes. Chem Mater. 2007;19:5937-45.

[175] Voet VSD, Hermida-Merino D, ten Brinke G, Loos K. Block copolymer route towards poly(vinylidene fluoride)/poly(methacrylic acid)/nickel nanocomposites. RSC Adv. 2013;3:7938-46.

[176] Borkar S, Sen A. Novel Fluoroalkene-Methyl Acrylate Copolymers by Atom Transfer Radical Polymerization. Macromolecules. 2005;38:3029-32.

[177] Dolbier WR, Rodriguez-Garcia V, Wu K, Angerhofer A, Hedhli L, Elsheikh M. Novel fluoropolymers formed by an unprecedented SRN1 condensation polymerization mechanism. J Fluorine Chem. 2008;129:991-3.

[178] Thirumurugan P, Matosiuk D, Jozwiak K. Click Chemistry for Drug Development and Diverse Chemical-Biology Applications. Chem Rev. 2013;113:4905-79. [179] Vukićević R, Vukovic I, Stoyanov H, Korwitz A, Pospiech D, Kofod G, Loos K, Brinke G t, Beuermann S. Poly(vinylidene fluoride)-functionalized single-walled carbon nanotubes for the preparation of composites with improved conductivity. Polym Chem. 2012;3:2261-5.

[180] Vukićević R, Hierzenberger P, Hild S, Beuermann S. Functionalization of carbon black nanoparticles with poly(vinylidene fluoride). J Polym Sci, Part A: Polym Chem. 2010;48:484754. 
[181] Vukicevic R, Schwadtke U, Schmucker S, Schafer P, Kuckling D, Beuermann S. Alkyneazide coupling of tailored poly(vinylidene fluoride) and polystyrene for the synthesis of block copolymers. Polym Chem. 2012;3:409-14.

[182] Guerre M, Semsarilar M, Totée C, Silly G, Améduri B, Ladmiral V. Self-assembly of poly(vinylidene fluoride)-block-poly(2-(dimethylamino)ethylmethacrylate) block copolymers prepared by CuAAC click coupling. Polym Chem. 2017;8:5203-11.

[183] Terzic I, Meereboer NL, Acuautla M, Portale G, Loos K. Electroactive materials with tunable response based on block copolymer self-assembly. Nat Commun. 2019;10:601. [184] Terzic I, Meereboer NL, Acuautla M, Portale G, Loos K. Tailored Self-Assembled Ferroelectric Polymer Nanostructures with Tunable Response. Macromolecules. 2019;52:354-64. [185] Terzic I, Meereboer NL, Loos K. CuAAC click chemistry: a versatile approach towards PVDF-based block copolymers. Polym Chem. 2018;9:3714-20.

[186] Huang H, Chen C, Lo S, Lin C, Chen S, Lin L. Identification of ionic aggregates in PVDFg-PSSA membrane by tapping mode AFM and HADDF STEM. Appl Surf Sci 2006;253:2685-9.

[187] Danks TN, Slade RCT, Varcoe JR. Comparison of PVDF- and FEP-based radiationgrafted alkaline anion-exchange membranes for use in low temperature portable DMFCs. J Mater Chem. 2002;12:3371-3.

[188] Liu F, Du C-H, Zhu B-K, Xu Y-Y. Surface immobilization of polymer brushes onto porous poly(vinylidene fluoride) membrane by electron beam to improve the hydrophilicity and fouling resistance. Polymer. 2007;48:2910-8.

[189] Cai T, Neoh KG, Kang ET. Poly(vinylidene fluoride) Graft Copolymer Membranes with "Clickable" Surfaces and Their Functionalization. Macromolecules. 2011;44:4258-68. 
[190] Kader MA, Kwak SK, Kang SL, Ahn J-H, Nah C. Novel microporous poly(vinylidene fluoride)-graft-poly(tert-butyl acrylate) electrolytes for secondary lithium batteries. Polym Int. 2008;57:1199-205.

[191] Albert A, Barnett AO, Thomassen MS, Schmidt TJ, Gubler L. Radiation-Grafted Polymer Electrolyte Membranes for Water Electrolysis Cells: Evaluation of Key Membrane Properties. ACS Appl Mater Interfaces. 2015;7:22203-12.

[192] Paronen M, Sundholm F, Ostrovskii D, Jacobsson P, Jeschke G, Rauhala E, Tikkanen P. Preparation of Proton-Conducting Membranes by Direct Sulfonation. 1. Effect of Radicals and Radical Decay on the Sulfonation of Poly(vinyl fluoride) Films. Chem Mater. 2003;15:4447-55. [193] Lienafa L, Monge S, Guillaneuf Y, Ameduri B, Siri D, Gigmes D, Robin J-J. Preparation of PVDF-grafted-PS involving nitroxides. Eur Polym J. 2018;109:55-63.

[194] Chen J, Tan S, Gao G, Li H, Zhang Z. Synthesis and characterization of thermally selfcurable fluoropolymer triggered by TEMPO in one pot for high performance rubber applications. Polym Chem. 2014;5:2130-41.

[195] Peng Q, Lu S, Chen D, Wu X, Fan P, Zhong R, Xu Y. Poly(vinylidene fluoride)-graftPoly(N-vinyl-2-pyrrolidone) Copolymers Prepared via a RAFT-Mediated Process and their Use in Antifouling and Antibacterial Membranes. Macromol Biosci. 2007;7:1149-59.

[196] McKee JR, Ladmiral V, Niskanen J, Tenhu H, Armes SP. Synthesis of StericallyStabilized Polystyrene Latexes Using Well-Defined Thermoresponsive Poly(Nisopropylacrylamide) Macromonomers. Macromolecules. 2011;44:7692-703.

[197] Guerre M, Ameduri B, Ladmiral V. One-pot synthesis of poly(vinylidene fluoride) methacrylate macromonomers via thia-Michael addition. Polym Chem. 2016;7:441-50. [198] Chen Y, Ying L, Yu W, Kang ET, Neoh KG. Poly(vinylidene fluoride) with Grafted 
Poly(ethylene glycol) Side Chains via the RAFT-Mediated Process and Pore Size Control of the Copolymer Membranes. Macromolecules. 2003;36:9451-7.

[199] Hester JF, Banerjee P, Won YY, Akthakul A, Acar MH, Mayes AM. ATRP of Amphiphilic Graft Copolymers Based on PVDF and Their Use as Membrane Additives. Macromolecules. 2002;35:7652-61.

[200] Akthakul A, Salinaro RF, Mayes AM. Antifouling Polymer Membranes with Subnanometer Size Selectivity. Macromolecules. 2004;37:7663-8.

[201] Inceoglu S, Olugebefola SC, Acar MH, Mayes AM. Atom transfer radical polymerization using poly(vinylidene fluoride) as macroinitiator. Des Monomers Polym. 2004;7:181-9. [202] Asatekin A, Menniti A, Kang S, Elimelech M, Morgenroth E, Mayes AM. Antifouling nanofiltration membranes for membrane bioreactors from self-assembling graft copolymers. $\mathbf{J}$ Membr Sci. 2006;285:81-9.

[203] Akthakul A, McDonald WF, Mayes AM. Noncircular pores on the surface of asymmetric polymer membranes: evidence of pore formation via spinodal demixing. J Membr Sci. 2002;208:147-55.

[204] Asatekin A, Mayes AM. Responsive Pore Size Properties of Composite NF Membranes Based on PVDF Graft Copolymers. Sep Sci Technol. 2009;44:3330-45.

[205] Zhang Z, Chalkova E, Fedkin M, Wang C, Lvov SN, Komarneni S, Chung TCM. Synthesis and Characterization of Poly(vinylidene fluoride) $g$-sulfonated Polystyrene Graft Copolymers for Proton Exchange Membrane. Macromolecules. 2008;41:9130-9.

[206] Lee JW, Cho HJ, Chun J, Kim KN, Kim S, Ahn CW, Kim W, Kim J-Y, Kim S-W, Yang C, Baik J M. Robust nanogenerators based on graft copolymers via control of dielectrics for remarkable output power enhancement. Science Adv. 2017;3:e1602902. 
[207] Samanta S, Chatterjee DP, Manna S, Mandal A, Garai A, Nandi AK. Multifunctional Hydrophilic Poly(vinylidene fluoride) Graft Copolymer with Supertoughness and Supergluing Properties. Macromolecules. 2009;42:3112-20.

[208] Kuila A, Chatterjee DP, Layek RK, Nandi AK. Coupled atom transfer radical coupling and atom transfer radical polymerization approach for controlled grafting from poly(vinylidene fluoride) backbone. J Polym Sci, Part A: Polym Chem. 2014;52:995-1008.

[209] Kuila A, Chatterjee DP, Maity N, Nandi AK. Multi-functional poly(vinylidene fluoride) graft copolymers. J Polym Sci Part A: Polym Chem. 2017;55:2569-84.

[210] He F, Luo B, Yuan S, Liang B, Choong C, Pehkonen SO. PVDF film tethered with RGDclick-poly(glycidyl methacrylate) brushes by combination of direct surface-initiated ATRP and click chemistry for improved cytocompatibility. RSC Adv. 2014;4:105-17.

[211] Holmberg S, Holmlund P, Wilén C-E, Kallio T, Sundholm G, Sundholm F. Synthesis of proton-conducting membranes by the utilization of preirradiation grafting and atom transfer radical polymerization techniques. J Polym Sci, Part A: Polym Chem. 2002;40:591-600.

[212] Sauguet L, Boyer C, Ameduri B, Boutevin B. Synthesis and Characterization of Poly(vinylidene fluoride)-g-poly(styrene) Graft Polymers Obtained by Atom Transfer Radical Polymerization of Styrene. Macromolecules. 2006;39:9087-101.

[213] Sauguet L, Ameduri B, Boutevin B. Radical copolymerization of vinylidene fluoride with 8-bromo-1H,1H,2H-perfluorooct-1-ene: microstructure, crosslinking and thermal properties. Macromol Chem Phys. 2007;208:1061-72.

[214] Tan S, Zhang Y, Niu Z, Zhang Z. Copper(0) Mediated Single Electron Transfer Controlled Radical Polymerization toward C-F Bonds on Poly(vinylidene fluoride). Macromol Rapid Commun. 2018;39:1700561. 
[215] Zhang M, Russell TP. Graft Copolymers from Poly(vinylidene fluoride-cochlorotrifluoroethylene) via Atom Transfer Radical Polymerization. Macromolecules. 2006;39:3531-9.

[216] Guan F, Yang L, Wang J, Guan B, Han K, Wang Q, Zhu L. Confined Ferroelectric Properties in Poly(Vinylidene Fluoride-co-Chlorotrifluoroethylene)-graft-Polystyrene Graft Copolymers for Electric Energy Storage Applications. Adv Funct Mater. 2011;21:3176-88. [217] Guan F, Wang J, Yang L, Tseng J-K, Han K, Wang Q, Zhu L. Confinement-Induced HighField Antiferroelectric-like Behavior in a Poly(vinylidene fluoride-co-trifluoroethylene-cochlorotrifluoroethylene)-graft-polystyrene Graft Copolymer. Macromolecules. 2011;44:2190-9. [218] Tan S, Li J, Zhang Z. Study of Chain Transfer Reaction to Solvents in the Initiation Stage of Atom Transfer Radical Polymerization. Macromolecules. 2011;44:7911-6.

[219] Seo JA, Kim YW, Roh DK, Shul YG, Kim JH. Proton conducting grafted/crosslinked membranes prepared from poly(vinylidene fluoride-co-chlorotrifluoroethylene) copolymer. Polym Advan Technol. 2011;22:1434-41.

[220] Hu X, Li J, Li H, Zhang Z. Synthesis and characterization of poly(vinylidene fluoride-cochlorotrifluoroethylene)-grafted-poly(acrylonitrile) via single electron transfer-living radical polymerization process. J Polym Sci, Part A: Polym Chem. 2012;50:3126-34.

[221] Hu X, Li J, Li H, Zhang Z. Cu(0)/2,6-bis(imino)pyridines catalyzed single-electron transfer-living radical polymerization of methyl methacrylate initiated with poly(vinylidene fluoride-co-chlorotrifluoroethylene). J Polym Sci Part A: Polym Chem. 2013;51:4378-88. [222] Zhu N, Hu X, Zhang Y, Zhang K, Li Z, Guo K. Continuous flow SET-LRP in the presence of $\mathrm{P}(\mathrm{VDF}-\mathrm{co}-\mathrm{CTFE})$ as macroinitiator in a copper tubular reactor. Polym Chem. 2016;7:474-80. [223] Wang J, Xie Y, Liu J, Zhang Z, Zhang Y. Towards high efficient nanodielectrics from 
linear ferroelectric P(VDF-TrFE-CTFE)-g-PMMA matrix and exfoliated mica nanosheets. Appl Surf Sci. 2019;469:437-45.

[224] Guan FX, Yuan ZZ, Shu EW, Zhu L. Fast discharge speed in poly(vinylidene fluoride) graft copolymer dielectric films achieved by confined ferroelectricity. Appl Phys Lett 2009;94:052907.

[225] Li J, Tan S, Ding S, Li H, Yang L, Zhang Z. High-field antiferroelectric behaviour and minimized energy loss in poly(vinylidene-co-trifluoroethylene)-graft-poly(ethyl methacrylate) for energy storage application. J Mater Chem. 2012;22:23468-76.

[226] Li W, Meng Q, Zheng Y, Zhang Z, Xia W, Xu Z. Electric energy storage properties of poly(vinylidene fluoride). Appl Phys Lett. 2010;96:192905.

[227] Li Y, Li L, Sun J. Bioinspired Self-Healing Superhydrophobic Coatings. Angew Chem Int Ed. 2010;122:6265-9.

[228] Tsang EMW, Zhang Z, Shi Z, Soboleva T, Holdcroft S. Considerations of Macromolecular Structure in the Design of Proton Conducting Polymer Membranes: Graft versus Diblock Polyelectrolytes. J Am Chem Soc. 2007;129:15106-7.

[229] Koh JH, Kim YW, Park JT, Min BR, Kim JH. Nanofiltration membranes based on poly(vinylidene fluoride- co- chlorotrifluoroethylene)- graft- poly(styrene sulfonic acid). Polym Adv Technol 2008;19:1643.

[230] Koh JK, Kim YW, Ahn SH, Min BR, Kim JH. Antifouling poly(vinylidene fluoride) ultrafiltration membranes containing amphiphilic comb polymer additive. J Polym Sci, Part B: Polym Phys. 2010;48:183-9.

[231] Kim YW, Choi JK, Park JT, Kim JH. Proton conducting poly(vinylidene fluoride-cochlorotrifluoroethylene) graft copolymer electrolyte membranes. J Membr Sci. 2008;313:315. 
[232] Park CH, Lee CH, Guiver MD, Lee YM. Sulfonated hydrocarbon membranes for mediumtemperature and low-humidity proton exchange membranes fuel cells (PEMFCs). Prog Polym Sci. 2011;36:1443-98.

[233] Yang ACC, Narimani R, Zhang Z, Frisken BJ, Holdcroft S. Controlling Crystallinity in Graft Ionomers, and Its Effect on Morphology, Water Sorption, and Proton Conductivity of Graft Ionomer Membranes. Chem Mater. 2013;25:1935-46.

[234] Park JT, Koh JH, Roh DK, Shul YG, Kim JH. Proton-conducting nanocomposite membranes based on P(VDF-co-CTFE)-g-PSSA graft copolymer and TiO2-PSSA nanoparticles Int J Hydrogen Energy. 2011;36:1820.

[235] Hwangbo K-H, Kim Y-J, Cho KY. Fabrication of protein-resistant blend based on PVDFHFP and amphiphilic brush copolymer made from PMMA and PEGMA. Appl Surf Sci. 2012;263:291-6.

[236] Kobayashi M, Higaki Y, Kimura T, Boschet F, Takahara A, Ameduri B. Direct surface modification of poly(VDF-co-TrFE) films by surface-initiated ATRP without pretreatment. RSC Adv. 2016;6:86373-84.

[237] Valade D, Boschet F, Ameduri B. Grafting polymerization of styrene onto alternating terpolymers based on chlorotrifluoroethylene, hexafluoropropylene, and vinyl ethers, and their modification into ionomers bearing ammonium side-groups. J Polym Sci, Part A: Polym Chem. 2010;48:5801-11.

[238] Patil Y, Bilalis P, Polymeropoulos G, Almahdali S, Hadjichristidis N, Rodionov V. A Novel Poly(vinylidene fluoride)-Based 4-Miktoarm Star Terpolymer: Synthesis and SelfAssembly. Mol Pharmaceutics. 2018;15:3005-9.

[239] Lopez G, Guerre M, Améduri B, Habas J-P, Ladmiral V. Photocrosslinked PVDF-based 
star polymer coatings: an all-in-one alternative to PVDF/PMMA blends for outdoor applications. Polym Chem. 2017;8:3045-9.

[240] Apostolides DE, Patrickios CS, Sakai T, Guerre M, Lopez G, Améduri B, Ladmiral V, Simon M, Gradzielski M, Clemens D, Krumm C, Tiller JC, Ernould B, Gohy J-F. Near-Model Amphiphilic Polymer Conetworks Based on Four-Arm Stars of Poly(vinylidene fluoride) and Poly(ethylene glycol): Synthesis and Characterization. Macromolecules. 2018;51:2476-88. [241] Yagi T, Tsuda N, Noguchi T, Sakaguchi K, Tanaka Y, Tatemoto M. EP1990/0422644 (assigned to Daikin Industries Ltd.); 1990.

[242] Améduri B, Boutevin B. Synthesis, properties and applications of fluorinated thermoplastic elastomers. Actualite Chimique. 2006:123-9.

[243] Patel R, Park JT, Lee WS, Kim JH, Min BR. Composite polymer electrolyte membranes comprising P(VDF-co-CTFE)-g-PSSA graft copolymer and zeolite for fuel cell applications. Polym Adv Technol. 2009;20:1146-51.

[244] Jana KK, Prakash O, Shahi VK, Avasthi DK, Maiti P. Poly(vinylidene fluoride-cochlorotrifluoro ethylene) Nanohybrid Membrane for Fuel Cell. ACS Omega. 2018;3:917-28. [245] Wang J-W, Shen Q-D, Yang C-Z, Zhang Q-M. High Dielectric Constant Composite of P(VDF-TrFE) with Grafted Copper Phthalocyanine Oligomer. Macromolecules. 2004;37:22948.

[246] Pester CW, Liedel C, Ruppel M, Böker A. Block copolymers in electric fields. Prog Polym Sci. $2017 ; 64: 182-214$.

[247] Durand N, Ameduri B, Takashima K, Ishida K, Horie S, Ueda Y. Vinylidene fluoride telomers for piezoelectric devices. Polym J. 2011;43:171-9.

[248] Noda K, Ishida K, Kubono A, Horiuchi T, Yamada H, Matsushige K. Molecular 
Ferroelectricity of Vinylidene Fluoride Oligomer Investigated by Atomic Force Microscopy. Jap J Appl Phys. 2001;40:4361-4.

[249] García-Iglesias M, de Waal BFM, Gorbunov AV, Palmans ARA, Kemerink M, Meijer EW. A Versatile Method for the Preparation of Ferroelectric Supramolecular Materials via Radical End-Functionalization of Vinylidene Fluoride Oligomers. J Am Chem Soc. 2016;138:6217-23.

[250] Xia F, Cheng Z-Y, Xu HS, Li HF, Zhang QM, Kavarnos GJ, Ting RY, Abdul-Sadek G, Belfield KD. High Electromechanical Responses in a Poly(vinylidene fluoride-trifluoroethylene-chlorofluoroethylene) Terpolymer. Adv Mater. 2002;14:1574-7. [251] Yang L, Allahyarov E, Guan F, Zhu L. Crystal Orientation and Temperature Effects on Double Hysteresis Loop Behavior in a Poly(vinylidene fluoride-co-trifluoroethylene-cochlorotrifluoroethylene)-graft-Polystyrene Graft Copolymer. Macromolecules. 2013;46:9698711.

[252] Yang L, Li X, Allahyarov E, Taylor PL, Zhang QM, Zhu L. Novel polymer ferroelectric behavior via crystal isomorphism and the nanoconfinement effect. Polymer. 2013;54:1709-28. [253] Meereboer NL, Terzić I, Saidi S, Hermida Merino D, Loos K. Nanoconfinement-Induced $\beta$-Phase Formation Inside Poly(vinylidene fluoride)-Based Block Copolymers. ACS Macro Lett. 2018;7:863-7.

[254] Goldbach JT, Amin-Sanayei R, He W, Henry J, Kosar W, Lefebvre A, O'brien G, Vaessen D, Wood K, Zerafati S. Commercial Synthesis and Applications of Poly(Vinylidene Fluoride). In: Ameduri B, Sawada H, editors. Fluorinated Polymer Volume 2: Applications. Cambridge: Royal Society of Chemistry; 2016, chapter 6, pp 127-157. [255] Barbosa J, Dias J, Lanceros-Méndez S, Costa C. Barbosa J, Dias J, Lanceros-Méndez S, 
Costa C. Recent advances in poly (vinylidene fluoride) and its copolymers for lithium-ion battery separators. Membranes. 2018;8:45.

[256] Costa CM, Silva MM, Lanceros-Mendez S. Battery separators based on vinylidene fluoride (VDF) polymers and copolymers for lithium ion battery applications. RSC Adv. 2013;3:11404-17.

[257] Xiao Q, Wang X, Li W, Li Z, Zhang T, Zhang H. Macroporous polymer electrolytes based on PVDF/PEO- $b$-PMMA block copolymer blends for rechargeable lithium ion battery. J Membr Sci. 2009;334:117-22.

[258] Kissa E. Fluorinated Surfactants and Repellents. Second ed. New York: Taylor \& Francis; 2001, p. 640.

[259] Park S-H, Ahn Y, Jang M, Kim H-J, Cho KY, Hwang SS, Lee J-H, Baek K-Y. Effects of methacrylate based amphiphilic block copolymer additives on ultra filtration PVDF membrane formation. Sep Purif Technol. 2018;202:34-44.

[260] Derry MJ, Fielding LA, Armes SP. Polymerization-induced self-assembly of block copolymer nanoparticles via RAFT non-aqueous dispersion polymerization. Prog Polym Sci. 2016;52:1-18.

[261] Penfold NJW, Yeow J, Boyer C, Armes SP. Emerging Trends in Polymerization-Induced Self-Assembly. ACS Macro Lett. 2019;8:1029-54.

[262] Guerre M, Semsarilar M, Godiard F, Ameduri B, Ladmiral V. Polymerization-induced self-assembly of PVAc-b-PVDF block copolymers via RAFT dispersion polymerization of vinylidene fluoride in dimethyl carbonate. Polym Chem. 2017;8:1477-87.

[263] Wu Y, Chen L, Sun X, Xu J, Gu G, Qian J. Self-assembly of poly(vinylidene fluoride)polystyrene block copolymers in solution: Effects of the length of polystyrene block and solvent 
compositions. J Saudi Chem Soc. 2017;21:713-9.

[264] Narayana KJ, Gupta Burela R. A review of recent research on multifunctional composite materials and structures with their applications. Materials Today: Proceedings. 2018;5:5580-90. [265] Bounor-Legaré V, Cassagnau P. In situ synthesis of organic-inorganic hybrids or nanocomposites from sol-gel chemistry in molten polymers. Prog Polym Sci. 2014;39:1473-97. [266] Prateek, Thakur VK, Gupta RK. Recent Progress on Ferroelectric Polymer-Based Nanocomposites for High Energy Density Capacitors: Synthesis, Dielectric Properties, and Future Aspects. Chem Rev. 2016;116:4260-317.

[267] Lee M, Koo T, Lee S, Min BH, Kim JH. Morphology and physical properties of nanocomposites based on poly(methyl methacrylate)/poly(vinylidene fluoride) blends and multiwalled carbon nanotubes. Polym Composites. 2015;36:1195-204.

[268] Safonov AI, Sulyaeva VS, Timoshenko NI, Kubrak KV, Starinskiy SV. Deposition of plasmon gold-fluoropolymer nanocomposites. Phys Lett A. 2016;380:3919-23.

[269] Elashmawi IS, Alatawi NS, Elsayed NH. Preparation and characterization of polymer nanocomposites based on PVDF/PVC doped with graphene nanoparticles. Results in Physics. 2017;7:636-40.

[270] Bouharras FE, Raihane M, Silly G, Totee C, Ameduri B. Core-shell structured poly(vinylidene fluoride)-grafted-BaTiO3 nanocomposites prepared via reversible additionfragmentation chain transfer (RAFT) polymerization of VDF for high energy storage capacitors. Polym Chem. 2019;10:891-904.

[271] Xie X, He Z-z, Qi X-d, Yang J-h, Lei Y-z, Wang Y. Achieving high performance poly(vinylidene fluoride) dielectric composites via in situ polymerization of polypyrrole nanoparticles on hydroxylated $\mathrm{BaTiO}_{3}$ particles. Chem Sci. 2019;10:8224-35. 
[272] Sawada H, Tashima T, Nishiyama Y, Kikuchi M, Goto Y, Kostov G, Ameduri B. Iodine Transfer Terpolymerization of Vinylidene Fluoride, $\alpha$-Trifluoromethacrylic Acid and Hexafluoro-propylene for Exceptional Thermostable Fluoropolymers/Silica Nanocomposites. Macromolecules. 2011;44:1114-24.

[273] Guo S, Yoshioka H, Kakehi H, Kato Y, Miura M, Isu N, Ameduri B, Sawada H.

Fluoroalkyl end-capped vinyltrimethoxysilane oligomer/anatase titanium oxide nanocomposites possessing photocatalytic activity even after calcination at $1000^{\circ} \mathrm{C}$. J Colloid Interface Sci. 2012;387:141-5.

[274] Durand N, Gaveau P, Silly G, Ameduri B, Boutevin B. Radical Grafting of Tetrafluoroethylene and Vinylidene Fluoride Telomers onto Silica Bearing Vinyl Groups. Macromolecules. 2011;44:6249-57.

[275] Durand N, Habas J-P, Boutevin B, Améduri B. Dispersion of silica nanoparticles bearing perfluorohexyl units into fluorinated copolymers. J Polym Sci, Part A: Polym Chem. 2015;53:1512-22.

[276] Voet VSD, Tichelaar M, Tanase S, Mittelmeijer-Hazeleger MC, ten Brinke G, Loos K. Poly(vinylidene fluoride)/nickel nanocomposites from semicrystalline block copolymer precursors. Nanoscale. 2013;5:184-92.

[277] Terzić I, Meereboer NL, Mellema HH, Loos K. Polymer-based multiferroic nanocomposites via directed block copolymer self-assembly. J Mater Chem C. 2019;7:968-76. [278] Soulestin T, Ladmiral V, Dos Santos FD, Ameduri B. Vinylidene Fluoride- and Trifluoroethylene-containing Fluorinated Electroactive Copolymers. How Does Chemistry Impact Properties? Prog Polym Sci. 2017;72:16-60.

[279] Jung C-H, Hwang I-T, Kuk I-S, Choi J-H, Oh B-K, Lee Y-M. Poly(acrylic acid)-Grafted 
Fluoropolymer Films for Highly Sensitive Fluorescent Bioassays. ACS Appl Mater Interfaces. 2013;5:2155-60.

[280] Yanai N, Uemura T, Uchida N, Bracco S, Comotti A, Sozzani P, Kodani T, Koh M,

Kanemura T, Kitagawa S. End-functionalization of a vinylidene fluoride oligomer in coordination nanochannels. J Mater Chem. 2011;21:8021-5.

[281] Xie H, Yang K-K, Wang Y-Z. Photo-cross-linking: A powerful and versatile strategy to develop shape-memory polymers. Prog Polym Sci. 2019;95:32-64.

[282] Sun H, Kabb CP, Sims MB, Sumerlin BS. Architecture-transformable polymers:

Reshaping the future of stimuli-responsive polymers. Prog Polym Sci. 2019;89:61-75.

[283] Eken GA, Acar MH. PVDF-based shape memory polymers. Eur Polym J. 2019;114:249-

54. 